\author{
Technische Universität München \\ Lehrstuhl für Medientechnik
}

\title{
Mobile Visual Location Recognition
}

\author{
Dipl.-Ing. Univ. Georg G. A. Schroth
}

Vollständiger Abdruck der von der Fakultät für Elektrotechnik und Informationstechnik der Technischen Universität München zur Erlangung des akademischen Grades eines

$$
\text { Doktor-Ingenieurs (Dr.-Ing.) }
$$

genehmigten Dissertation.

Vorsitzender:

Prüfer der Dissertation:
Univ.-Prof. Dr.-Ing. Klaus Diepold

1. Univ.-Prof. Dr.-Ing. Eckehard Steinbach

2. Univ.-Prof. Dr.-Ing. Rainer Lienhart Universität Augsburg

Die Dissertation wurde am 20.03.2013 bei der Technischen Universität München eingereicht und durch die Fakultät für Elektrotechnik und Informationstechnik am 17.06.2013 angenommen. 


\title{
Mobile Visual Location Recognition
}

\author{
Dipl.-Ing. Georg Schroth
}

July 22, 2013 



\section{Acknowledgments}

This dissertation presents large parts of my research at the Institute for Media Technology (LMT) at the Technische Universität München (TUM). My research has been supported in part by the space agency of the German Aerospace Center with funds from the Federal Ministry of Economics and Technology on the basis of a resolution of the German Bundestag under the reference 50NA1307.

First of all, I would like to express my gratitude to my supervisor Prof. Eckehard Steinbach for inviting me into his team and giving me the exceptional opportunity of starting a new research project without prior funding. I would like to thank Prof. Steinbach for his ongoing support, his academic advice, and the space to follow my own vision in which he guided me as my mentor.

Further, I would like to thank Prof. Rainer Lienhart for accepting to be the second reviewer of this dissertation and Prof. Klaus Diepold for heading the committee.

My appreciation also goes to Dr. Oliver Funke and Norbert Neu at the German Aerospace Center who believed in the ideas and vision of a visual indoor localization system and granted the funds for of the NAVVIS project.

My sincere appreciation also goes to all my current and former colleagues of the LMT NAVVIS group, especially to Robert Huitl, Sebastian Hilsenbeck, Julius Kammerl, and Florian Schweiger. They contributed considerably to this dissertation and many of the achievements presented would not have been possible without their support. I would also like to thank all my students, especially Dominik van Opdenbosch, who contributed to this dissertation. My appreciation also goes to the professional administrative support provided by Ingrid Jamrath, Dr. Martin Maier and Gabriele Kohl. They always kindly and competently helped me in every administrative matter I had.

Further, I would like to thank Prof. Bernd Girod for inviting me to the Information Systems Laboratory at Stanford. Thanks also go out to my close collaborators in the lab, David Chen, Frank Chen, Sam Tsai, Man Cheung, Vijay Chandrasekhar, and Gabriel Takacs.

In this context I would like to express my gratitude to Prof. Per Enge, Todd Walter and the whole team of the Stanford GPS Lab for the inspiring conversations during the last years.

My final and most sincere thanks go to my beloved Tamara, my parents, my grandmother, and my brother Christoph who always supported me in every possible way and encouraged me to follow my own way.

July 2013

Georg Schroth 


\section{Abstract}

Pulling out a road map or asking for directions to an address has long since become a thing of the past. Thanks to GPS, our smart phones do not only show us the shortest path but also allow us to bypass traffic jams. Indoors, however, we do not receive much help from our powerful smart phones as GPS is virtually not available. Direction signs pointing in all directions can be found at almost every meter in buildings like airports, malls, train stations, hospitals, and universities. Indoor positioning could support us in our daily life in form of navigation systems and location based services like vicinity searches. Using the smart phone as a virtual tour guide, the museum visit could become an entirely different experience via augmented reality apps.

To this date most attention is paid to Wi-Fi based indoor localization systems, which have been studied for more than a decade. These systems, however, are error-prone and require at least four to five Wi-Fi hot spots in the vicinity to allow for a room level accuracy. Thus, additional hot spots need to be installed throughout the building, which poses a major obstacle for the widespread adoption.

To avoid the need for costly infrastructure, this thesis proposes a vision based localization system. Inspired by human orientation, images recorded by smart phones are used as a visual fingerprint of the current environment and matched to a previously recorded and geo-tagged reference database. By employing content based image retrieval techniques, the search for the visually most similar reference image and thus the current location can be performed in fractions of a second. This thesis addresses four major challenges in developing a mobile visual localization system that ultimately might enable location based services indoors:

To reduce the computational complexity when matching the current recordings of a mobile device to the reference database, a novel quantization structure is introduced that robustly quantizes visual features into so-called visual words. The quantization into visual words allows us to apply well studied text retrieval approaches to the image recognition problem.

Due to the rapidly changing field of view and user attention, very low system latency is essential for location based services to be perceived as useful. Standard client server architectures, where visual features are uploaded to the server to perform the localization, result in significant transmission delays. This thesis presents a novel approach that eliminates this delay by preloading selected reference information, so-called partial visual vocabularies, to the smart phone. This enables the smart phone to perform the localization locally without contacting the server for a limited area. At the same time, the approach efficiently integrates prior knowledge about the location into the matching process, which leads to a significant increase in retrieval performance.

Visual localization in poorly textured indoor environments is challenging due to the limited number of visual cues. Most distinctive features, however, can be found in the form of text characters. This thesis introduces an approach to exploit this important source of information without the need for optical character recognition which is computationally expensive and error-prone. To this end, a novel text detector based on maximally stable extremal regions 
is presented, which facilitates a robust yet low complexity localization of text in natural images. By exploiting not only the visual appearance but also the order of the detected characters, a highly distinctive visual feature description can be formed without increasing the computational complexity.

For most location based services, meter level accuracy as well as orientation estimation is required. This, however, would require to record reference images at all possible locations, which is hardly possible within a reasonable mapping time. This thesis introduces the socalled virtual view approach, which computes virtual reference images at every meter and at 16 different orientations. The approach projectively transforms actually recorded reference images to the virtual view's location. Hence, this approach is able to determine the position and orientation of images at locations where no reference images have actually been captured. Finally, with a maximum distance of $0.5 \mathrm{~m}$ between query and reference images, view point invariant features are no longer required and are replaced by low complexity features, which significantly reduces the computational load at the smart phone.

Altogether, this thesis demonstrates the feasibility of mobile visual location recognition in real world scenarios with today's smart phones. Nevertheless, several challenges are yet to be solved to increase robustness and availability and to advance visual indoor localization to a mature technology. 


\section{Contents}

Abbreviations and Acronyms $\quad$ ix

$\begin{array}{lll}1 & \text { Introduction } & 1\end{array}$

1.1 Localization in Downtown Environments . . . . . . . . . . . . . . . . . . . . 1

1.2 Concept and Challenges of Vision Based Localization . . . . . . . . . . . . . . 3

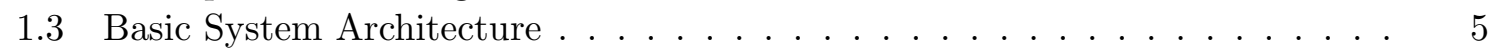

1.4 Contributions and Organization of this Thesis . . . . . . . . . . . . 6

$\begin{array}{lll}2 & \text { Related Work } & 9\end{array}$

2.1 Visual Odometry . . . . . . . . . . . . . . . . . . . . . . . . 9 9

2.1.1 Sequential Bayesian SLAM . . . . . . . . . . . . . . . . . . . 9 9

$2.1 .2 \quad$ Keyframe SLAM $\ldots \ldots \ldots \ldots \ldots$

2.2 Content Based Image Retrieval based Location Recognition . . . . . . . . . . 12

$2.2 .1 \quad$ Feature Extraction $\ldots \ldots \ldots \ldots \ldots$. . . . . . . . . . . . . . 14

$2.2 .2 \quad$ Bag-of-Features based Image Retrieval . . . . . . . . . . . . . . . . . . 17

$2.2 .3 \quad$ Visual Word Quantization and Indexing . . . . . . . . . . . . . . 23

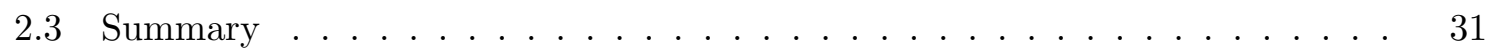

3 Multiple Hypothesis Vocabulary Tree 33

3.1 Related Work . . . . . . . . . . . . . . . . . . . . . . . . . . 34

3.2 Quantization structure . . . . . . . . . . . . . . . . . . . . . . 35

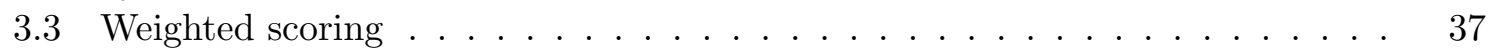

3.4 Summary $\ldots \ldots \ldots \ldots \ldots \ldots$

4 System Aspects in Mobile Visual Location Recognition $\quad 41$

4.1 Feature Extraction and Selection . . . . . . . . . . . . . . . . . . . . . . . 41

4.1 .1 Query Image . . . . . . . . . . . . . . . . . . . . . . . . 41

$4.1 .2 \quad$ Database . . . . . . . . . . . . . . . . . . . . . . . . 42

4.2 Coarse Prior Knowledge on Query Location . . . . . . . . . . . . . . . . . 43

4.3 Experimental Evaluation of the Basic Location Recognition Architecture. . . $\quad 45$

5 Elimination of Network Delay and Integration of Prior Knowledge on the Location 47

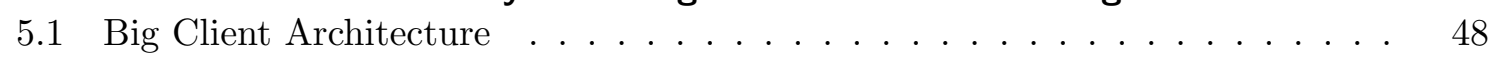

5.2 Partial Visual Vocabularies $\ldots \ldots \ldots \ldots \ldots$

$5.2 .1 \quad$ Composing the Partial Visual Vocabularies . . . . . . . . . . . 51

5.2 .2 Particle Filter Based Partial Vocabularies . . . . . . . . . . . . . . . . 53

5.3 Experimental Evaluation . . . . . . . . . . . . . . . . . . . 54

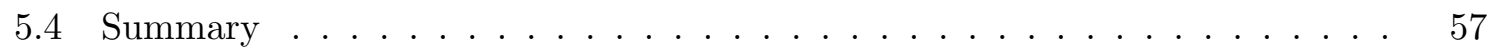

6 Visual Phrase Based Content Based Image Retrieval 59

6.1 Text Detection in Natural Images . . . . . . . . . . . . . . . . . . . 60 
$6.1 .1 \quad$ Edge-enhanced Maximally Stable Extremal Regions (EMSER) . . . . 63

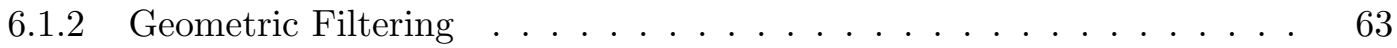

6.1 .3 Finding Stroke Width by Distance Transform . . . . . . . . . . . . . . 63

6.1 .4 Text Line Formation and Word Separation . . . . . . . . . . . . . . . 65

6.1 .5 Experimental Results _. . . . . . . . . . . . . . . . 66

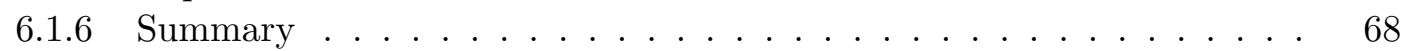

6.2 Visual Phrase based CBIR . . . . . . . . . . . . . . . . . . . . . . . . . . . 69

6.2 .1 Related Work . . . . . . . . . . . . . . . . . . . . . . . . . 69

6.2 .2 Detecting Visual Phrases $\ldots \ldots \ldots$. . . . . . . . . . . . . 71

6.2 .3 Visual Phrase based Retrieval . . . . . . . . . . . . . . . . . . . . 73

6.2 .4 Information Theoretic Model . . . . . . . . . . . . . . . . . . 77

6.2 .5 Combined N-grams . . . . . . . . . . . . . . . . . . . . . . . 79

6.3 Experimental evaluation $\ldots \ldots \ldots \ldots \ldots \ldots \ldots \ldots$

6.3 .1 Door Sign Recognition . . . . . . . . . . . . . . . . . . . . . 87

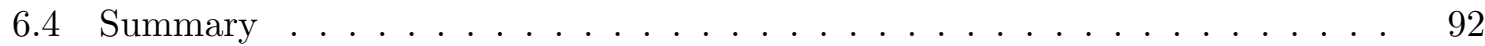

\begin{tabular}{llll}
\hline & Virtual Views for Meter-Accurate Localization and Orientation Assignment & 95
\end{tabular}

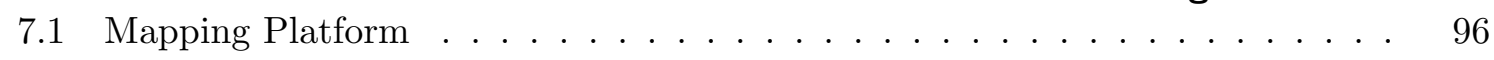

7.1 .1 Outlook to Upcoming Mapping Platform . . . . . . . . . . . . . . . . . 98

7.2 Virtual View generation . . . . . . . . . . . . . . . . . . 104

7.3 Integration of Binary Features . . . . . . . . . . . . . . . . . . 107

7.4 Localization Results $\ldots \ldots \ldots \ldots$. . . . . . . . . . . . . . . . 111

7.5 Summary . . . . . . . . . . . . . . . . . . . . . . . 114

$\begin{array}{lll}8 & \text { Conclusions and Outlook } & 117\end{array}$

\begin{tabular}{ll}
\hline List of Figures & 121
\end{tabular}

\begin{tabular}{ll}
\hline List of Tables & 127
\end{tabular}

\begin{tabular}{lr}
\hline Bibliography & 129
\end{tabular} 


\section{Abbreviations and Acronyms}

2D two-dimensional

3D three-dimensional

3G 3rd generation mobile telecommunications

AKM Approximate K-means

ANN Approximate Nearest Neighbor Search

AP Average Precision

ATM Automated Teller Machine

BBF Best Bin First

BKM Binary k-means

BoF Bag-of-Features

BRIEF Binary Robust Independent Elementary Features

BRISK Binary Robust Invariant Scalable Keypoints

CC Connected Component

CBIR Content-Based Image Retrieval

CHoG Compressed Histogram of Gradients

CV Computer Vision

DOF Degree of Freedom

DSLR Digital single-lens reflex camera

EMSER Edge-enhanced Maximally Stable Extremal Region

EKF Extended Kalman Filter

FAST Features from Accelerated Segment Test

FOV Field of View

fps frames per second

GLOH Gradient Location and Orientation Histogram

GPS Global Positioning System 
GSM Global System for Mobile Communications

HKM Hierarchical k-means tree

HMI Human-Machine Interface

ICDAR International Conference on Document Analysis and Recognition

IDF Inverse Document Frequency

IMU Inertial Measurement Unit

iSAM incremental Smoothing and Mapping

KNN K Nearest Neighbor Search

LBS Location Based Services

LSH Locality Sensitive Hashing

mAP mean Average Precision

MHVT Multiple Hypotheses Vocabulary Tree

MPEG Moving Pictures Expert Group

MSE Mean Squared Error

MSER Maximally Stable Extremal Region

NN Nearest Neighbor

OCR Optical Character Recognition

ORB Oriented FAST and Rotated BRIEF

PTAM Parallel Tracking and Mapping

RANSAC Random Sample Consensus

RFID Radio-frequency identification

SLAM Simultaneous localization and mapping

SfM Structure from Motion

SIFT Scale-invariant feature transform

SURF Speeded Up Robust Features

SVM Support Vector Machine

SWT Stroke Width Transform

TF Term Frequency

TOF Time of Flight 
UWB Ultra Wide Band

VLAD Vector of Locally Aggregated Descriptors

VT Vocabulary Tree

VW Visual Word

WGC Weak Geometric Consistency Check

WiFi Wireless Fidelity

WLAN Wireless Local Area Network 



\section{Introduction}

The situation is all too familiar: Just arrived at a foreign airport, we are looking for the fastest way downtown to catch an important appointment. The signs, however, show in all directions except to the taxi stand. While outdoors, location-based services (LBS) guide us through our daily life in form of tourist guides, mobile yellow pages, navigation systems, car sharing programs, and many more, indoors we do not receive much help from our powerful mobile devices, even for our most basic needs. These could include searching for the next ATM, finding the way in complex building structures, or even making an emergency call that transmits information on the current location.

The combination of information on the place, time and further context, however, enables a broad variety of applications which go beyond navigational tasks or vicinity searches. Using the smart phone as a virtual tourist guide, the museum visit could become an entirely different experience: By pointing the phone at an exhibit, details are highlighted on the screen and a video with background information is played back. Related exhibits that could be interesting to the visitor are suggested afterwards.

At the airport an indoor positioning system could indicate personalized Duty-Free offers on the way to the gate or help to find the shortest security line. Dynamic information like waiting times at a queue or last-minute deals, however, would be of great benefit not only for the user but also to the service provider to raise client satisfaction as well as to apply real-time marketing by analysing the customer flow.

There exists a tremendous variety of location based services and while the most interesting ones could be imagined to happen indoors, exactly here location and orientation information is still not easily available to enable them.

\subsection{Localization in Downtown Environments}

While satellite navigation systems can provide sufficient positioning accuracy, a clear view to at least four satellites is required, limiting its applicability to outdoor scenarios with few obstacles. Most interesting location-based services, however, could be provided in densely populated environments, which include urban canyons and indoor scenarios. To illustrate the localization performance of today's technology in these scenarios, Figure 1.1 shows the GPS recordings (black line) of an iPhone 4 while driving with a car through downtown San Francisco. Although a state-of-the-art Assisted-GPS Broadcom chip is used, the phone mounting ensures best signal reception, and a motion model is applied to filter out large deviations, the localization error is in the range of $50-100 \mathrm{~m}$. This is caused by multipath effects, which are even more severe if the user is traveling on the sidewalks and not in the middle of the street. In these environments, an initial positioning can take up to $40 \mathrm{~s}$. Ground truth in Figure 1.1 has been recorded with the aid of a high precision IMU and wheel odometry fused with the GPS signal. Clearly, the error is largest in the financial district, which is characterized by a dense collection of tall buildings. 


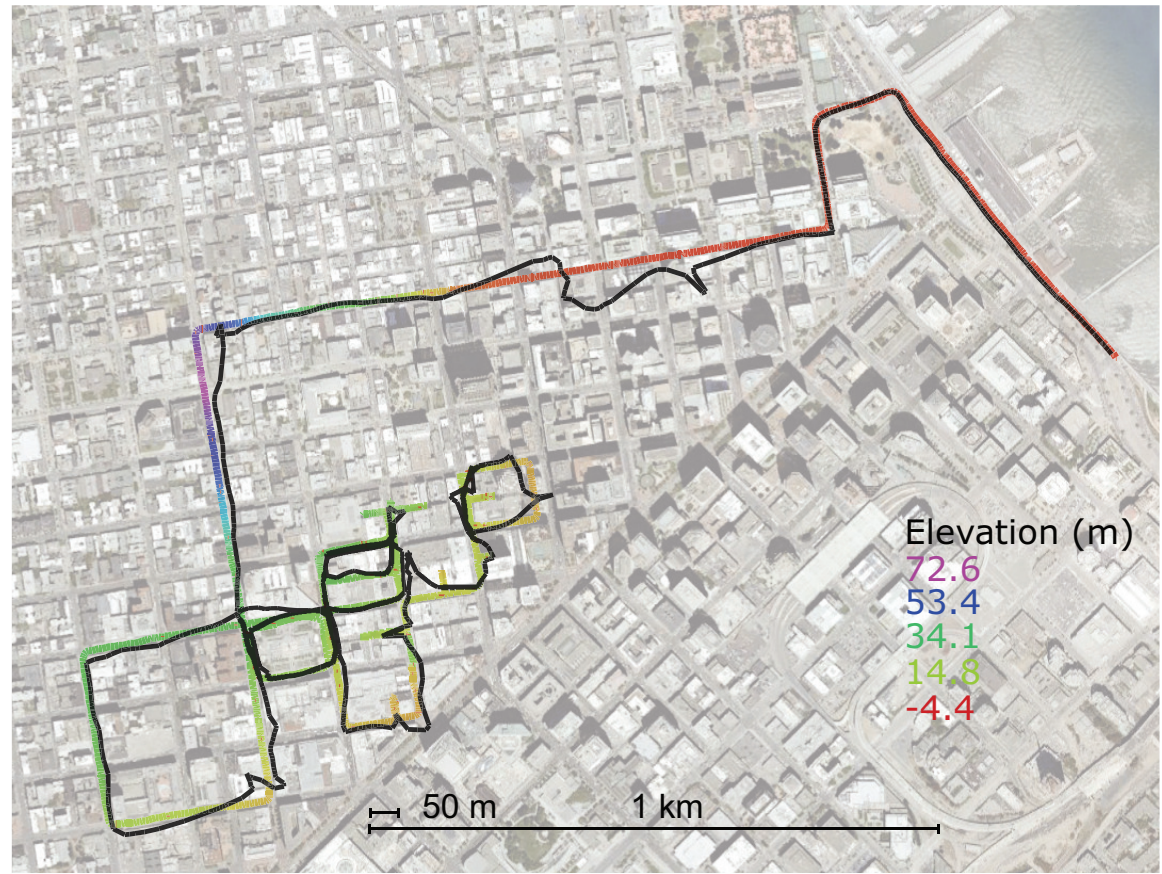

Figure 1.1: Track of video recordings in downtown San Francisco. Ground truth is shown as a colored path (color corresponds to elevation level), whereas the GPS recordings of the iPhone 4 are shown in black. (Reproduced from [SHC+11], (c) 2011 IEEE)

As GPS is virtually not available in indoor environments and the localization accuracy in urban canyons is insufficient, alternative positioning mechanisms, which can complement the available systems, are required.

During the last decade, a large body of work in the field of Wi-Fi signal based indoor positioning has been conducted. These approaches typically base upon triangulation, in which the distance to a known base station is measured by means of, e.g., Time of Flight (TOF) or signal strength. To allow for a precise location, not only the $3 \mathrm{D}$ world coordinates of the base station have to be known but also a detailed model of the building needs to be available. This is required to account for signal attenuation and multipath effects, which severely impair the localization results. To avoid the complex modeling of these effects, most state-of-the-art systems are based on radio signal fingerprints. Here, an offline mapping stage is conducted in which location coordinates and the respective signal strengths from nearby base stations are recorded. Thus, for every location visited during mapping, a vector of signal strengths can be formed which serves as a fingerprint. During the actual localization, various approaches to match the current fingerprint with the database of previously recorded signal strength vectors can be performed. These include $\mathrm{k}$ nearest neighbor search, classifiers, or probabilistic frameworks. Nevertheless, also fingerprint based systems cannot account for dynamic objects like persons or doors which alter the signal strength not only in their direct vicinity but also in large areas. Thus, several base stations (5-10) have to be in range at every possible location within a building to allow for a localization accuracy of 3-5 m. This level of accuracy would be sufficient for vicinity searches within buildings, while more sophisticated location based services like navigation require an accuracy of about $1 \mathrm{~m}$. Typically, however, the number of available Wi-Fi hotspots per location in public buildings, like museums or 
airports, is in the range of 2-3 hotspots. This is clearly insufficient and may allow for a localization accuracy of about $10-15 \mathrm{~m}$ at best.

While Wi-Fi signals have a range of about 50-100 m, Bluetooth $(2-10 \mathrm{~m})$ and passive RFID tags $(1-2 \mathrm{~m})$ have a much lower range. Thus even more beacons need to be installed. Such a dense distribution, however, would in turn allow for precise and robust localization in areas like narrow corridors.

In contrast to Wi-Fi, Ultra Wide Band (UWB) signals are hardly affected by signal attenuation, due to their very short signal pulse (1 ns). Further, Time of Flight based approaches can be applied as the pulse is easily sorted out among all reflections. Hence, a cm level or even higher accuracy can be achieved.

A good overview of existing radio signal based indoor localization systems and an evaluation with respect to accuracy, availability, complexity, robustness, scalability, and cost can be found in [LDBL07.

\subsection{Concept and Challenges of Vision Based Localization}

This thesis aims at a novel vision based localization system that uses images recorded on the mobile device in combination with a visual reference database. In contrast to radio based indoor localization systems, no infrastructure, which grows in complexity with the size of the environment, is required. Low investment costs are essential to facilitate the widespread adoption of a localization system and in turn to enable location based services indoors. LBS do not only rely on precise location information (meter level) but also require orientation information to determine the user's actual field of view (FOV). Further, they also benefit from information about the objects in the FOV, like exhibits, store names, trademarks, etc., which can be derived from the images the user is intentionally recording. In the following, the challenges of a vision based indoor localization system are discussed.

A typical usage scenario would be a person entering an airport with the intention to board a plane. Once the user has entered his intention on his mobile device, e.g., by taking a photo of the ticket, he would expect the system to instantly display directions where he will find the check-in counter. While the device can use prior knowledge, obtained from the last GPS signals, Wi-Fi, or GSM based localisation systems, a first pose estimate within the building has to be determined within seconds based on the visual data recorded by the mobile device. Low complexity localization approaches are required to avoid excessively draining the battery. It is important for the performance of a visual localization system that a distinctive part of the environment is recorded and the device is not pointing at the floor. By illustrating information on the display using augmented reality approaches, the user is virtually looking through the display and observes the surroundings with overlaid directions (and other relevant information) to the check-in counter, as illustrated in Figure 1.2. While it can be assumed that the user is not pointing the device at a white wall to localize himself, the system still has to cope with sparsely textured indoor environments like plain hospital corridors.

For most location based services the localization accuracy should be within 1-5 $\mathrm{m}$ as humans (unlike robots) do not require a $\mathrm{cm}$ level positioning to orient themselves in an unknown environment. The requirements for navigational applications can be relaxed by providing landmark based directions to the user. Hence, a graph based map of a building is more applicable to routing applications than a metric representation. The current location uncertainty also 


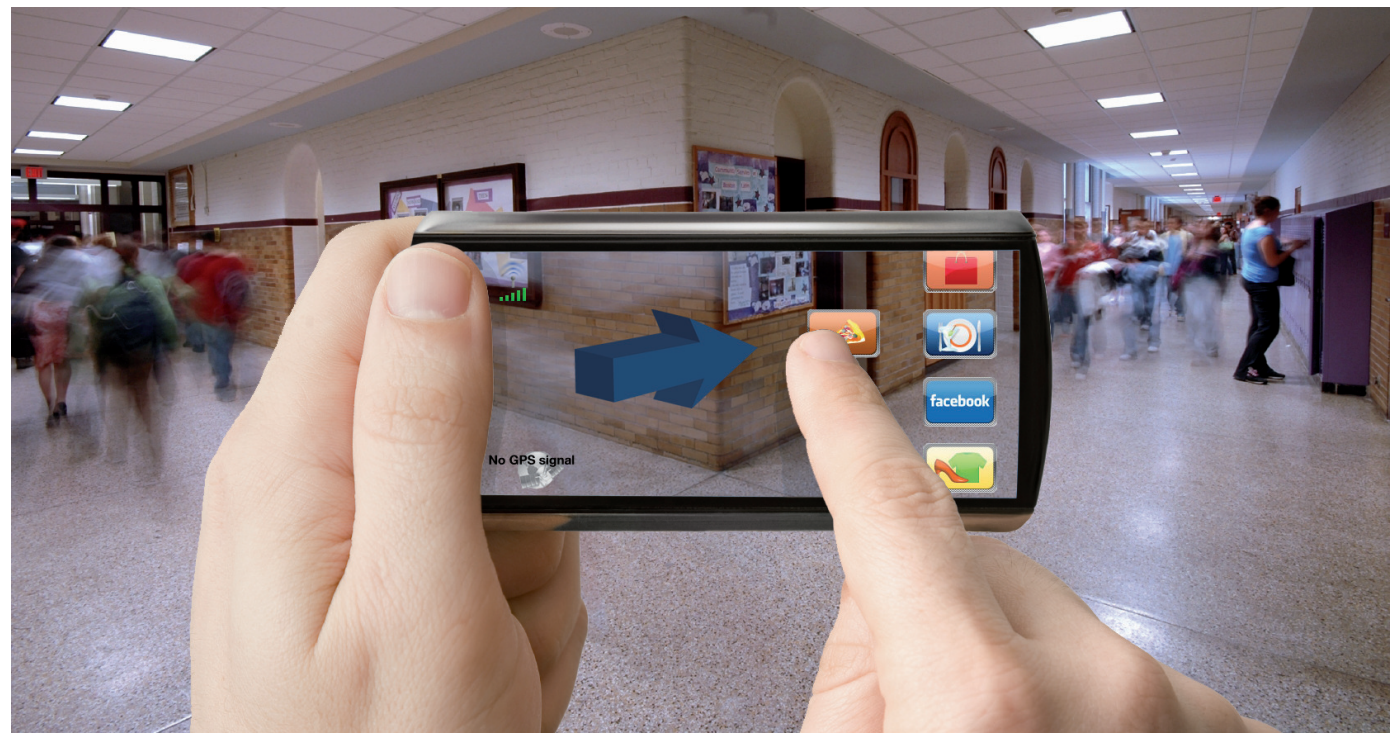

Figure 1.2: Mockup of a mobile visual localization system in an indoor environment.

has to have an impact on the way the directions and further information are presented to the user.

Once the user knows how to get to the check-in counter, the device can no longer be assumed to be held upright such that images with distinctive content of the environment are recorded. Rather, the device will be put into a pocket or bag. However, the user still expects to be informed by the device if he is not on the right track via sound or vibration signals. Thus, the localization system has to make use of other, less reliable and less precise sensors built into the mobile device, like Wi-Fi, compass, and IMU based pedometer to estimate the relative position within a limited time frame.

After checking in, the device would inform the user about the time required for passing the security check and on how much time there is left for browsing through duty free shops. It would estimate the intention of the user based on aggregated information of his schedule, shopping preferences, etc. and show only relevant hints on the display that are in the field of view of the device to reduce the cognitive load. While in navigational applications an orientation accuracy of about $30^{\circ}$ would be sufficient, the requirements of augmented reality applications might be more stringent. A content analysis in the field of view of the device is required to estimate what the user is currently focusing on which allows the system to provide solely relevant information and location based services to the user.

The reference data required for visual localization within a building needs to be captured within a few days at low cost. This is essential for a widespread adoption of a visual localization system. Thus, a basic panoramic camera has to be sufficient to allow a non-expert to record sufficient visual data of a building, forming the basis of the reference data. Based on this, a graphical representation of the building has to be drawn, which is required to enter location related services and information into the system. However, as the visual appearance of a building is dynamic, e.g., as a result of changing advertisements or announcements, the system has to adapt to these changes without requiring the generation of a new complete set of 
reference data. Rather, the visual data recorded by the mobile devices during self-localization should be used to continuously update the reference data.

\subsection{Basic System Architecture}

While in robotic applications, optical sensors have been used for more than a decade to perform localization within buildings [ $\left.\mathrm{TBB}^{+} 99\right]$, the high descriptiveness of image recordings has been exploited only to a limited extend. In most applications, features with very limited distinctiveness have been processed that provide only a weak hint on the actual location. Fusing the individual contributions over time using probabilistic filters, like the particle filter, allows a robot to narrow down the location estimate after traversing, e.g., a corridor.

One of the most prominent requirements of an indoor positioning system, however, is the need for an instant global localization. Recent progress in the field of content based image retrieval (CBIR) allows us to form a fine-grained description of an image while at the same time being robust against small changes caused by occlusions or different perspectives. With the increased distinctiveness of these image descriptions, visually similar images can be found in fractions of a second in extensive datasets. Section 2.2 provides an introduction into the active research area of content based image retrieval and an evaluation of state-of-the-art approaches. In a mobile context, CBIR is mainly applied in product recognition tasks, in which the recorded object has to be identified and related information is displayed. The most prominent commercial applications in this field are Google Goggles and Amazon SnapTell Goo12c, Ama12.

By using the images recorded by our smart phone as visual fingerprints of the environment and matching them to an existing database of reference images with known position, CBIR allows us to perform an instant global localization in a very natural way.

In the case of outdoor localization, several geo-referenced databases like Google Street View [Goo12d] or Microsoft Street-Side views [Mic12] already exist and cover even remote streets in most countries. Outdoors, GPS combined with wheel odometry allows for an easy georeferencing of the database images. Indoors, the situation is more complicated as no external reference exists. Simultaneous localization and mapping algorithms, introduced in Section 2.1, need to be applied to solve the chicken-and-egg problem. Google already started to record panoramic views indoors, however, so far they are available mostly as a proof of concept in selected museums. Section 7.1 introduces the NAVVIS mapping trolley, which was used to generate the datasets for the indoor experiments of this thesis.

With a visual reference database available for a given location, the basic system architecture of a visual localization system could be designed as shown in Figure 1.3 . Due to the memory requirements of the image retrieval structure, CBIR typically has to be performed at a server. Hence, visual features are extracted from the recorded query image and transmitted to the server. CBIR identifies the visually most similar reference images among the database and returns their global location.

When considering indoor environments with repetitive and sparse textures, a single query image can be insufficient and results in an ambiguous retrieval outcome indicating multiple possible locations. To resolve the ambiguity, absolute location estimates obtained from CBIR have to be set in a relation by estimating the distance traveled between them. This can be done using visual odometry approaches that track features to compute estimates of the 


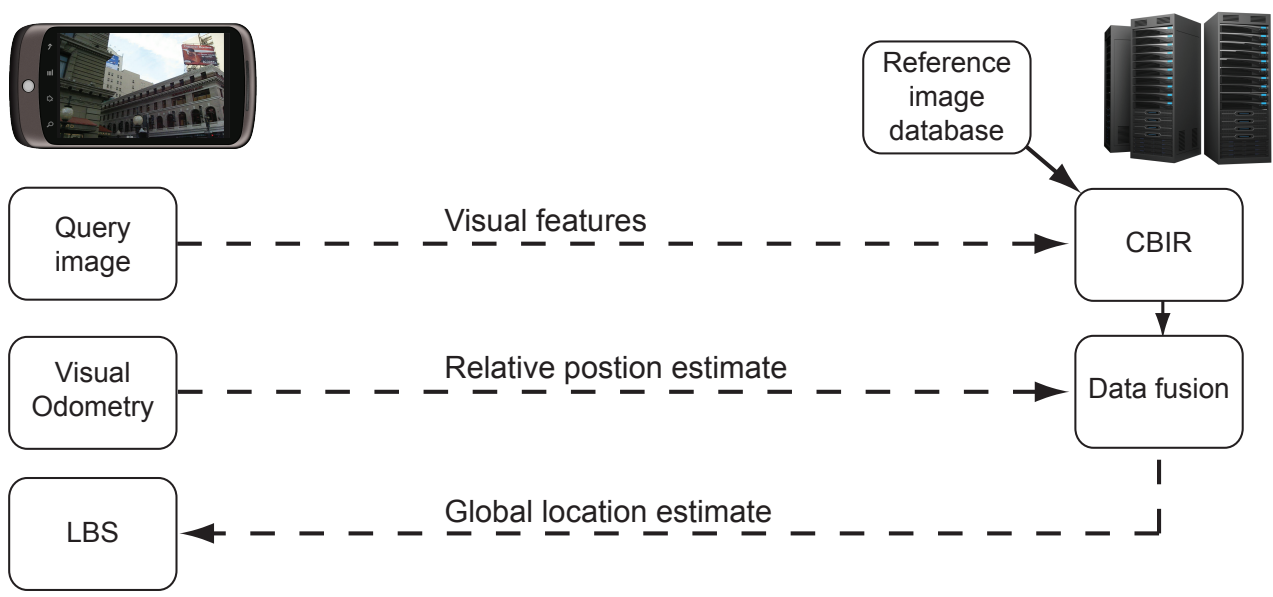

Figure 1.3: Basic system architecture of a mobile visual localization system.

relative pose change. Section 2.1 provides an introduction to visual odometry and discusses state-of-the-art systems.

The fusion of global and relative location estimates of CBIR and visual odometry is performed either at the server or at the client depending on the available bandwidth and the required update rate. This can be done using a particle filter to model the multimodal belief distribution. Finally, the global pose estimate is sent to the mobile device to enable location based services.

\subsection{Contributions and Organization of this Thesis}

The goal of this thesis is to address the challenges discussed in Section 1.2 developing a vision based localization system that ultimately might enable location based services indoors.

The specific major contributions are as follows: To reduce the computational complexity when matching the current image recordings of a mobile device with a database of georeferenced imagery, the so-called Multiple Hypothesis Vocabulary Tree (MHVT) has been developed and is presented in Chapter 3. The MHVT allows for robust quantization of, e.g., 1000 visual feature descriptors within $12 \mathrm{~ms}$ on a Nexus One smart phone with a $1 \mathrm{GHz}$ CPU. The quantization allows us to apply well studied text retrieval approaches to the image recognition problem. Retrieval performance comparable to significantly more complex quantization algorithms is accomplished by applying an overlapping buffer at each quantization step, an adaptive clustering approach, and by integrating the probability of correct quantization into the image similarity calculation.

As described in Section 1.2, ideally, a user is notified about an LBS in the very moment the camera records the corresponding store, exhibit, trademark, etc. Due to the rapidly changing field of view and dynamically changing user attention, very low system latency is essential for LBS to be perceived as useful. In the basic system architecture, described in Section 1.3, features extracted on a mobile device are uploaded to the server which performs the actual image matching. This results in a significant delay and increases the network load 
when considering multiple users in the system. In Chapter 5, an approach that eliminates the network delay by preloading relevant parts of the quantization structure to the mobile device by exploiting the typically 5 times faster downlink is described and validated. This allows us to perform the localization within a limited area locally on the mobile device without waiting for responses from the server. Retrieval performance is significantly increased by identifying relevant parts of the quantization structure based on the uncertainty about the location of the client. This way, prior knowledge is efficiently integrated into the image matching process. Based on compressed feature sets, infrequently uploaded from the mobile device, the server estimates the client location and its uncertainty by fusing consecutive query results using a particle filter.

Distinctive visual cues are of central importance for image retrieval applications, in particular, in the context of visual location recognition. While in indoor environments typically only few distinctive features can be found, outdoors dynamic objects and clutter significantly impair the retrieval performance. An approach which exploits text, a major source of information for humans during orientation and navigation, without the need for error-prone optical character recognition is presented in Chapter 6. As a first step, text in natural images needs to be robustly detected and localized. To this end, a novel low complexity, yet high quality text detector based on maximally stable extremal regions (MSER) [MCUP04] is introduced in Section 6.1. Once characters are detected, the so-called visual phrase based approach in Section 6.2 describes the respective texture using robust visual feature descriptors like SURF [BETG08]. By quantizing these feature descriptors into a set of several hundred centroids (termed visual words), we consider the distinctive appearance of the characters rather than reducing the set of possible features to the size of an alphabet. Writings in images are transformed to strings of visual words termed visual phrases, which provide significantly improved distinctiveness when compared to individual features. An approximate string matching is performed using $\mathrm{N}$-grams (substrings of length $N$ ), which allows us to cope with large datasets. This approach results in a significant improvement of the retrieval performance while reducing the size of the database by two orders of magnitude when compared to the state-of-the-art. Its low computational complexity makes the approach particularly suited for mobile image retrieval applications.

To assess the performance of the mobile visual localization system in indoor environments, a mapping platform was developed to establish large scale visual indoor datasets (see Section 7.1). Images as well as a $3 \mathrm{D}$ point cloud of the environment are recorded while localization and mapping is based on fusing a laser range sensor with wheel odometry. This allowes for a high precision mapping of tracks of more than $2 \mathrm{~km}$ length.

For most location based services, meter level accuracy as well as orientation estimation is required. However, to allow for a reasonable mapping time, reference images cannot be recorded at any possible location but only along a trajectory through the building. Performing a pose refinement based on the matched reference views via pairwise feature matching and triangulation is a time consuming task and does not allow for real-time applications. To this end, the novel so-called virtual view based approach was developed and is presented in Section 7.2. Virtual reference views are generated along a regular grid with $1 \mathrm{~m}$ spacings and at 16 different orientations. The approach relies on knowledge about planar regions present in the scene, and projectively transforms reference images to the virtual views' location. Hence, this approach is able to determine the position and orientation of images at locations where no reference images have been captured. This in turn, allows for meter level localization, 
increases the location recognition performance in general as it copes well with perspective distortion, and speeds up the mapping process.

While visual features like SIFT and SURF [Low04, BETG08] are robust against affine transformations and achieve a high detection repeatability, their computational complexity poses a major hurdle on the way to low latency location recognition. Recently several low complexity, binary features have been proposed [CLSF10, RRKB11, LCS11, AOV12. The Binary Robust Feature (BRIEF) by Calonder et. al [CLSF10] is about 40 times faster than SURF and requires only one half of the memory to be stored. However, this comes at the cost of lower descriptive power and, most importantly, low invariance against affine transformations. Section 7.3 shows how the concept of virtual views combined with BRIEF allows us to achieve retrieval results comparable to SIFT and SURF. Due to the dense sampling of virtual views, invariance against substantial affine transformations is no longer required. In fact, the limited invariance of BRIEF increases the distinctiveness with respect to the viewpoint. A quantization structure, adapted to the 256 dimensional binary descriptor vector, is proposed which allows for fast and robust visual word assignment. Finally, it is shown how this binary feature quantizer integrates seemlessly with the low latency approach of Chapter 5.

Parts of this thesis have been published in $\left[\mathrm{SANH}^{+} 11, \mathrm{CTS}^{+} 11, \mathrm{SHH}^{+} 11, \mathrm{SHH}^{+} 12, \mathrm{SHC}^{+} 11\right.$, $\left.\mathrm{SHAA}^{+} 12 \mathrm{~b}, \mathrm{SHAA}^{+} 12 \mathrm{a}, \mathrm{TCC}^{+} 11, \mathrm{HSH}^{+} 12 \mathrm{a}, \mathrm{HSH}^{+} 12 \mathrm{~b}\right]$. 


\section{Related Work}

This chapter serves as an introduction of the two major modules of the basic architecture, visual odometry and content based image retrieval (CBIR). Further, state-of-the-art in both research fields is discussed and evaluated.

\subsection{Visual Odometry}

In order to improve localization accuracy, the relative movement between reference points (i.e. where absolute location estimation is possible) needs to be estimated using odometry. To this end, different solutions have been proposed, ranging from pedometers [CP06] and inertial sensors [RAK09] to tracking using monocular or stereo cameras [NNB04, [JL06], or a combination of multiple sensors [NWSS11. As today's cell phones typically come with a single camera, we will concentrate in the following on monocular visual odometry.

Camera-based (visual) odometry can be understood as a special case of visual SLAM (Simultaneous Localization and Mapping). SLAM refers to the problem of estimating the pose of a robot in an initially unknown environment, while at the same time building a map of the environment. In the following a brief overview of the two basic concepts of visual SLAM is provided.

\subsubsection{Sequential Bayesian SLAM}

SLAM is usually treated as a state estimation problem, where the state vector contains the pose (location and orientation) as well as the locations of features. Initially, the map is empty and the pose of the mobile device is initialized to the prior, e.g., the origin of the coordinate system. When the first camera frame arrives, salient points (corners, SIFT features, etc.) are extracted and added to the state vector. Having been observed in a single image only, a feature can be located anywhere on a line in the 3D world, i.e., its distance from the camera is unknown. In other words, the camera is a bearing-only sensor, measuring the angle of incidence of the ray of light emitted by a feature onto the image plane. The uncertainty about the distance to the optical center of the camera has to be modeled in the state vector in order to efficiently incorporate more information as further observations of the same feature become available. Further, there is also uncertainty associated with the pose of the mobile device, which calls for a probabilistic approach to the SLAM problem.

The first successful application of a probabilistic filtering method to the SLAM problem has been demonstrated in [Dav03, DRMS07. The authors use an Extended Kalman Filter $(\mathrm{EKF})$ to incorporate measurements into the state vector and thereby estimate the pose of the camera as well as the map. In the EKF framework, the probability distributions of the state variables and the measurements are assumed to be Gaussian and can therefore conveniently be represented by their means and (co-)variances. This way, new features, where the uncertainty about the depth is large, start with a high variance, which will incrementally be reduced when 
further measurements provide more insight about a feature's depth. This system allows the construction of sparse room-sized maps in real-time $(30 \mathrm{~Hz})$ using monocular vision.

A major drawback of the approach described above is the parametrization of features, i.e., how the information about their location and associated uncertainty is stored in the state vector. Dav03, DRMS07] use a straight-forward parametrization with X, Y and Z coordinates in the world coordinate system. However, for newly initialized features that are very distant from the camera and exhibit only little parallax, the depth uncertainty is not represented well by a Gaussian distribution as implied by the EKF framework. Several methods have been proposed to circumvent this problem, usually by deferring the use of new features until the uncertainty approaches Gaussian shape. This special treatment of new features is generally undesirable, especially when taking into account that very distant features are highly reliable sources for measuring bearing. A remedy to this problem is the inverse depth parametrization proposed by [CDM08], which has since been adopted by the majority of authors. The key concept is that by initializing features in a coordinate system local to the camera position, and storing the inverse depth (1/depth) instead of the depth, the associated uncertainty more accurately resembles a Gaussian and can therefore be used in an EKF framework. This parametrization consists of the location of the camera when the feature was first observed, two angles defining a directional vector from the camera to the feature, and the inverse depth. When the uncertainty about a feature's depth has reduced, the simpler X,Y,Z parametrization can be used. Consequently, features are converted from the inverse depth to the X,Y,Z parametrization as soon as enough information about their position has been accumulated.

\subsubsection{Keyframe SLAM}

In contrast to filtering approaches as described in the previous section on Sequential Bayesian $S L A M$, a slightly different approach to solve the Visual SLAM problem has emerged from research in the area of Structure from Motion, where Bundle Adjustment is the method of choice. By extracting as much correspondence information as possible from a few key frames (every $n^{\text {th }}$ frame can be considered as a key frame) and applying global optimization based on projective geometry, both the pose of the camera and the position of the features can be calculated. There are various ways to select key frames from a video stream. Sliding windows have been used in $\mathrm{MDS}^{+} 06$, NNB04, but spatially distributed key frames as used in [KM07, KM08, allow for drift-free operation over a long time.

A comparison of the two fundamentally different approaches for monocular SLAM is given in [SMD10]. The authors conclude (with some reservations), that key frame optimization is superior compared to filtering in terms of accuracy per computing time. Methods that combine both approaches in order to take advantage of their individual strengths are iSAM [KRD08], FrameSLAM [KA08], and the stereo SLAM system of [MSC $\left.{ }^{+} 09\right]$.

In contrast to conventional key frame SLAM algorithms, the Parallel Tracking and Mapping (PTAM) approach by Klein and Murray [KM07] separates the tracking of image features for pose updates from the mapping part, where trackable features are collected to build a threedimensional map of the local environment (see Figure 2.1). This allows for an update of the camera pose at frame-rate, while expensive optimization techniques for the mapping can be executed at a lower rate and when computational resources are available. As a result, PTAM is eligible for mobile applications, whereas conventional SLAM approaches are prohibitively expensive due to their (at least) quadratic complexity in the number of observations. 

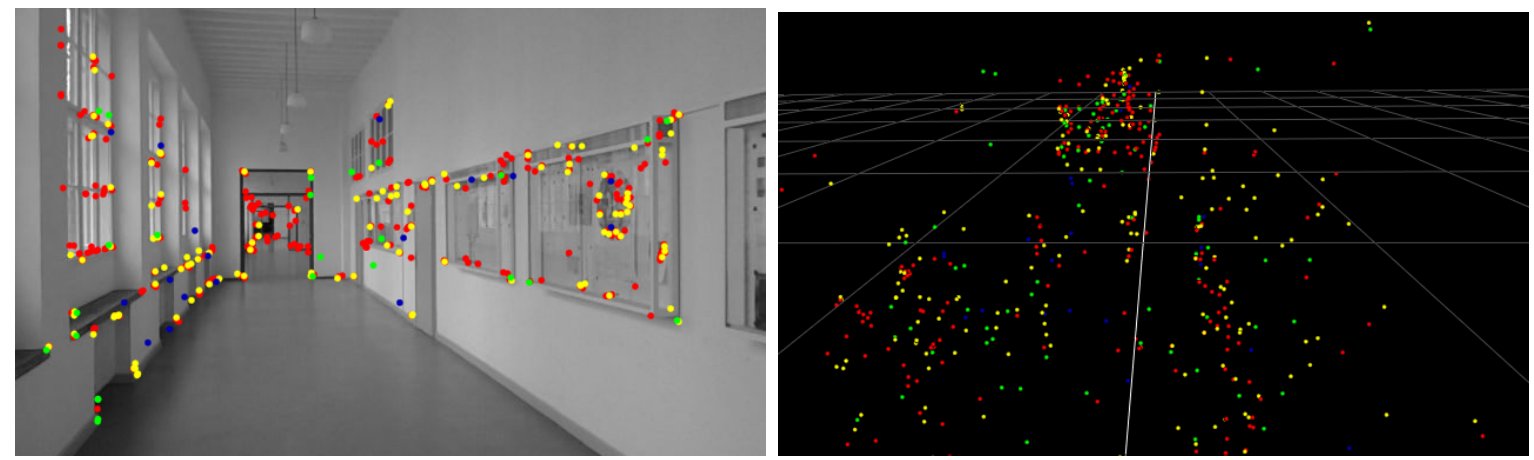

Figure 2.1: Left: A frame with feature points that are tracked by PTAM. The color of a point indicates the size of the feature in the image. Right: The corresponding three-dimensional point cloud as it appears in the reconstructed map. The 3D positions of the points are very noisy. The estimated ground plane is indicated by the grid lines. (Reproduced from [HMH+12], (C) 2012 IEEE)

The mapping part of PTAM operates key frame based, i.e., whenever a frame contains a large number of new observations that have been tracked, it is inserted into the map. Hence, every feature is stored within a key frame and the map comprises a sparse set of key frames. This allows for the use of bundle adjustment, which jointly optimizes the three-dimensional positions of observations and the six-dimensional poses of key frames. The optimized 3D positions of the features are, in turn, used during tracking for subsequent pose updates.

In contrast to SLAM, visual odometry does not aim at creating a globally consistent map of the environment, but uses local maps instead. PTAM can be easily converted into a visual odometry system by considering only key frames within a given vicinity for the bundle adjustment. Features that have been observed on distant key frames are discarded after a grace period.

Thus, PTAM provides a robust basis for a monocular visual odometry system running on mobile devices. The structure of the algorithm leads to comparatively low computational complexity while generating accurate position and orientation estimates with fairly small errors due to drift. However, an inherent disadvantage of monocular sensor systems is the inability to observe the true scale of position data relative to a metric coordinate system. Further, if no reference data is available to fix the scale, a problem called scale drift arises, where the scale is not only unknown, but deviates over time.

To enable anytime localization and navigation in indoor environments, it is therefore necessary to include additional information in order to scale the visual odometry to metric coordinates. Recent work exploits inertial data to perform metric reconstruction, i.e., the unknown visual scale is determined by incorporating inertial measurements [KS11, WS11. Kneip et al. describe an approach of this nature in [KWS11. They analyze delta-velocities obtained from both visual pose estimates and inertial measurements to solve for the unknown scale factor.

Further challenges are rapid movements of either the camera or objects in the scene that cause motion blur and thus decimate the number of visible features to a degree that the system loses tracking. PTAM incorporates a basic recovery mechanism, i.e., it stops adding new key frames to the map when tracking quality is considered poor and tries to match the 


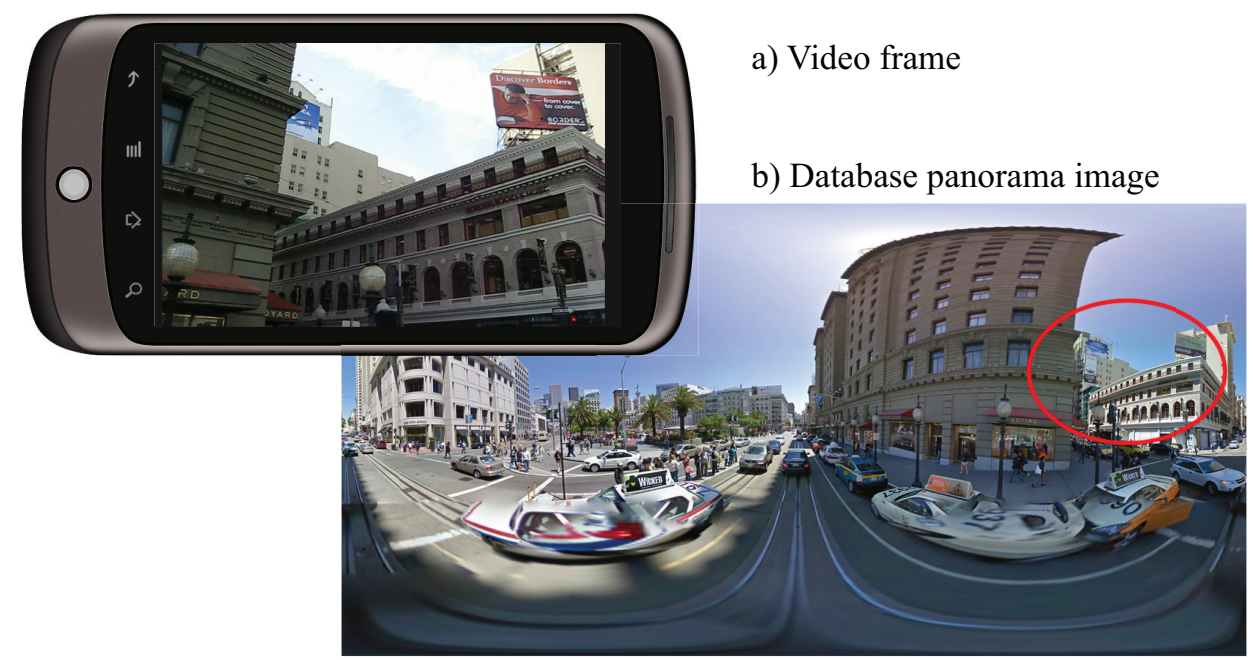

Figure 2.2: Google Street View panorama matched to a low resolution video recording (Union Square, San Francisco) using the MHVT-based location recognition system proposed in Chapter 3. (Reproduced from [SHC 11], (C) 2011 IEEE)

scene with previous key frames instead. However, recovery takes time and might only make sense in some cases, for instance, when large objects occlude the camera only temporarily (e.g., opening doors, people walking by). By contrast, if also the camera has moved in the meantime, it is unlikely that recovery succeeds.

Thus, the state-of-the-art in visual odometry hardly allows for tracking over long distances, i.e., more then $50 \mathrm{~m}$, in realistic scenarios. However, when combined with scale estimation and recovery techniques, it can efficiently resolve ambiguous global pose estimates obtained from content based image retrieval approaches.

\subsection{Content Based Image Retrieval based Location Recognition}

The main challenge for visual localization is to rapidly and accurately search for images related to the current recording in a large geo-referenced database. This task, known as Content Based Image Retrieval (CBIR), has been an area of intensive research for the last few decades [DJLW08]. Objects, recorded at different size, pose, and with varying background have to be distinctively described and efficiently retrieved from a database. The application of CBIR to location recognition complicates these requirements as will be discussed in the following.

As illustrated in Figure 2.2, images captured with a mobile device are used to retrieve the spatially closest image from a georeferenced dataset. This could, for instance, include the $360^{\circ}$ panoramic images from Google Street View Goo12d, which can be fetched from the web and are used throughout the following chapters. Typically, only sparse reference data can be assumed. For instance, Street View panoramas are available online with varying interpanorama distances, typically in the range of 12 to $17 \mathrm{~m}$. The three exemplary panoramas in Figure 2.3 illustrate the difficulties of matching spatially distant images, i.e., images with a large baseline between them. Whereas distant buildings can be well associated among the 
a

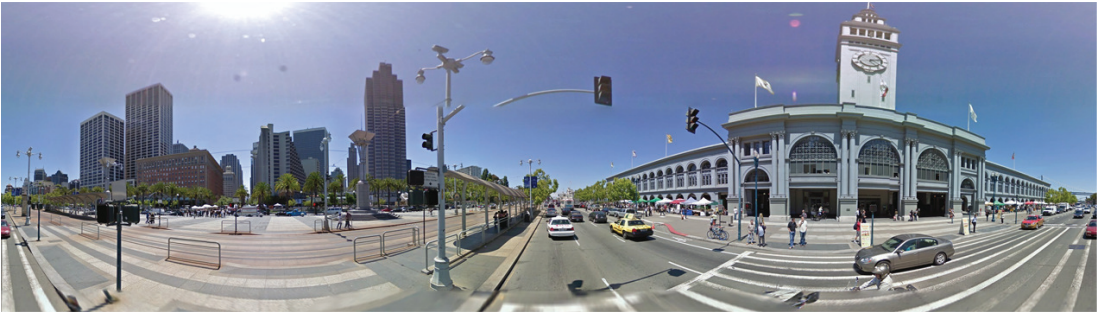

b

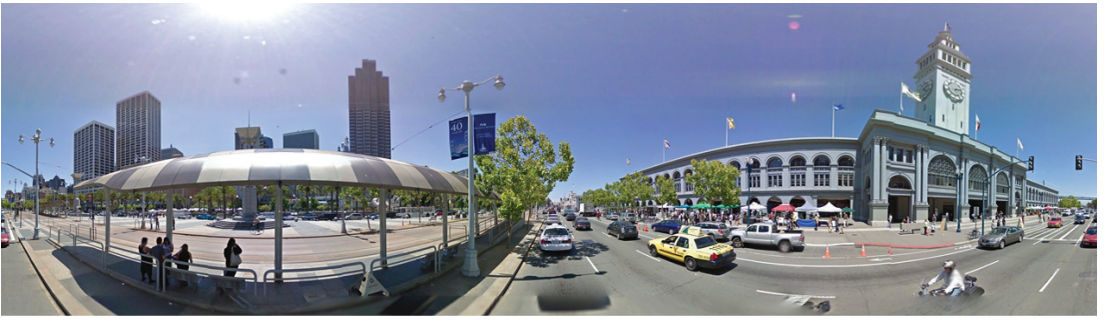

C

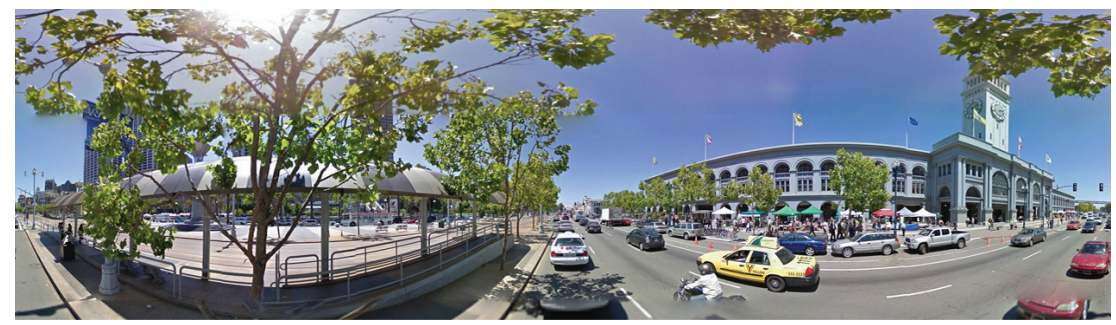

Figure 2.3: Sample images from the Google Street View dataset of San Francisco; available panoramas are on average $12.6 \mathrm{~m}$ apart from each other.

views, close objects like the train station or the tree are difficult to match even for a human observer. The description of distinct objects is complicated due to the three-dimensional structure of the environment and the resulting occlusions and overlaps. Further, different lighting conditions between the query and database image, which cause shadows and reflections, can change the visual appearance of the scene. Also, both query and database images typically contain dynamic objects, like cars or pedestrians, which lead to significant differences between matching views. As advertisements or even buildings alter over time and seasons change the appearance dramatically, a dynamic update process for the database is required. Due to the properties of mobile device cameras, query images are typically affected by motion blur and provide a limited field of view, which makes it difficult to match them against high resolution panoramas. Additionally, limitations on the processing power, battery capacity, and network performance require low complexity approaches on the mobile device and efficient communication including data compression.

Finally, very low retrieval times are an essential prerequisite for most LBS due to the rapidly changing field of view of the mobile device caused by user motion and constantly changing user attention. On the other hand, vague prior knowledge on the location of the mobile device can be always assumed, e.g., derived from Cell-IDs, which should be exploited to increase precision, reduce complexity and limit the requirements on the scalability of the approaches.

In the following, the state-of-the-art in visual mobile location recognition using so-called Bag-of-Features (BoF) based image retrieval algorithms is discussed with a focus on outdoor localization where the existence of georeferenced databases can be assumed and extensive 

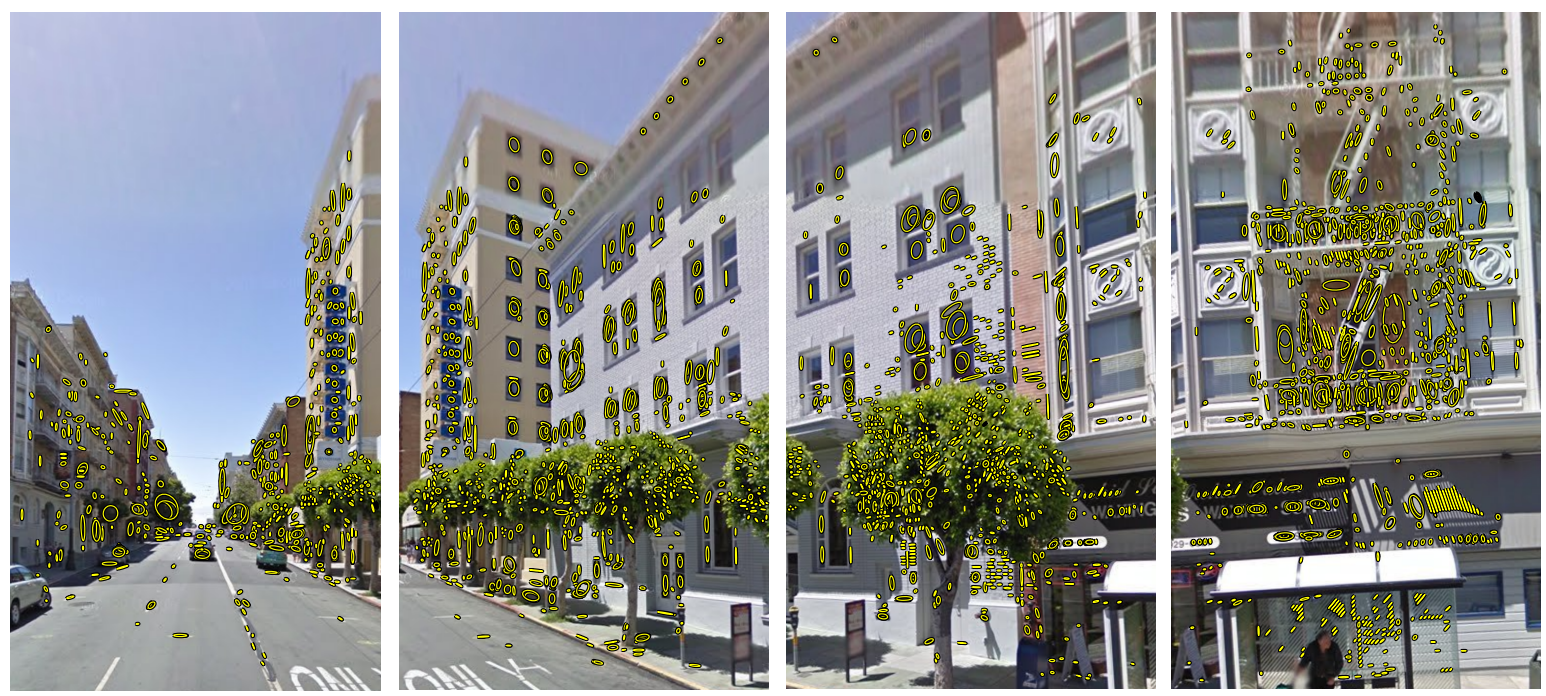

Figure 2.4: Sections of a Google Street View Panorama recorded in San Francisco. Yellow ellipses are fitted around detected MSER [MCUP04] regions. (Reproduced from [SHC+11], (C) 2011 IEEE)

datasets are accessible for experiments. As we go along, the challenges that should be addressed to advance the state-of-the-art in this promising research field are highlighted.

\subsubsection{Feature Extraction}

To match the current recording of the environment against a database of reference images and to retrieve the visually most similar ones, we need to find a way to efficiently compute their similarity. As mentioned above, in location recognition applications this similarity measure needs to be robust against changes with respect to the view point, occlusions, different lighting conditions, shadows, reflections, etc. Clearly, a simple pixelwise subtraction and computation of the sum of squared differences (SSD) does not meet these requirements as even small changes in the viewpoint would have a huge impact on this similarity measure. To cope with these affine transformations, early approaches compared images based on their color histogram. While this image signature is invariant against affine transformations, the distinctiveness of a color histogram is very limited and cannot cope with extensive databases.

In contrast to these global image descriptors, local features, which each consider only a small patch of the image, are less affected by perspective changes. Typically more than 300 local image patches are used to represent the complete image. In the case that a part of the image has changed, only a fraction of the local image features is affected, which makes the description of an image via local features robust against occlusions and dynamic objects. The similarity of two images is computed by determining the number of matching local features.

To select local features within an image, so-called interest point detectors are applied. They essentially search for salient regions, like corners, blobs, or regions of high contrast to their background. This is important to repeatedly detect the same interest points or regions in two images of the same scene recorded from different view points and at different lighting conditions. 
In the last few years, various feature detectors have emerged, which provide some invariance with respect to image scale changes, illumination conditions [Low04, BETG08] and affine transformations [MS04, MCUP04]. These properties are of particular importance in location recognition. Mikolajczyk et al. provide a comprehensive evaluation of affine region detectors in $\mathrm{MTS}^{+}$05] and conclude that Maximally Stable Extremal Regions (MSER) MCUP04] perform best in most cases. MSERs can be thought of as connected components (CCs), where all pixels inside the detected region have either higher or lower intensity than all pixels on the boundary. These could be windows, letters, or trademarks, as illustrated in Figure 2.4. In this figure, yellow ellipses are fitted around the detected interest regions based on their mean and standard deviation. By warping these elliptic image patches to circles with a predefined radius, the detected regions are normalized with respect to their scale and look the same from different perspectives. This process allows for some invariance against affine transformations.

For most experiments, best retrieval results have been obtained by combining the complimentary detectors Hessian-Affine [MS04], which detects interest points, and MSER detecting interest regions.

Unfortunately, these interest point / region detectors come with high computational complexity. As the Hessian-Affine detector requires at least four times more computations than the MSER detector, it is reasonable to employ only the latter in mobile application. Optimized implementations like the approach proposed in [NS08] allow us to process an image of $640 \mathrm{x} 480$ pixels in $50 \mathrm{~ms}$ on a $2 \mathrm{GHz}$ CPU, which can be further improved with the aid of parallel processing approaches on the GPU of the mobile device. Tracking these regions and computing additional MSERs only from the previously unseen boundary textures allows for a 5-6 fold speedup [TCGP09, DB06].

Once interest regions or points are detected and localized, the image texture in its vicinity needs to be compactly and distinctively described to allow us to determine corresponding local features. These feature descriptors should be robust against perspective changes, image orientation (in plane rotation), illumination, and small deviations in the interest point localization. This is typically achieved by building normalized histograms of the intensity gradients contained within the respective image patch. This can be illustrated by means of the well known speeded-up robust feature (SURF) descriptor [BETG08] outlined in Figure 2.5. As a first step the intensity gradients within the image patch defined by the interest point / region detector are computed. To achieve invariance against varying image orientations (in plane rotations) the dominant gradient direction (red arrow) is computed and the image patch is rotated accordingly. The patch is subdivided into $4 \mathrm{x} 4$ subregions, each containing 25 local gradient vectors. For each sub region, the sum of the gradients along the $\mathrm{x}$ and $\mathrm{y}$ axis as well as the sum of the absolute gradients is computed, as shown in Equation 2.1, which form four entries of the 64 dimensional descriptor vector ( $4 \mathrm{x} 4$ subregions with 4 entries each).

$$
\mathbf{x}_{i, j}=\left(\begin{array}{c}
\sum d x \\
\sum|d x| \\
\sum d y \\
\sum|d y|
\end{array}\right)
$$

Figure 2.6 shows the resulting descriptor entries for three different image intensity distributions. The contribution of each of the 25 local gradient vectors to these 4 descriptor vector 


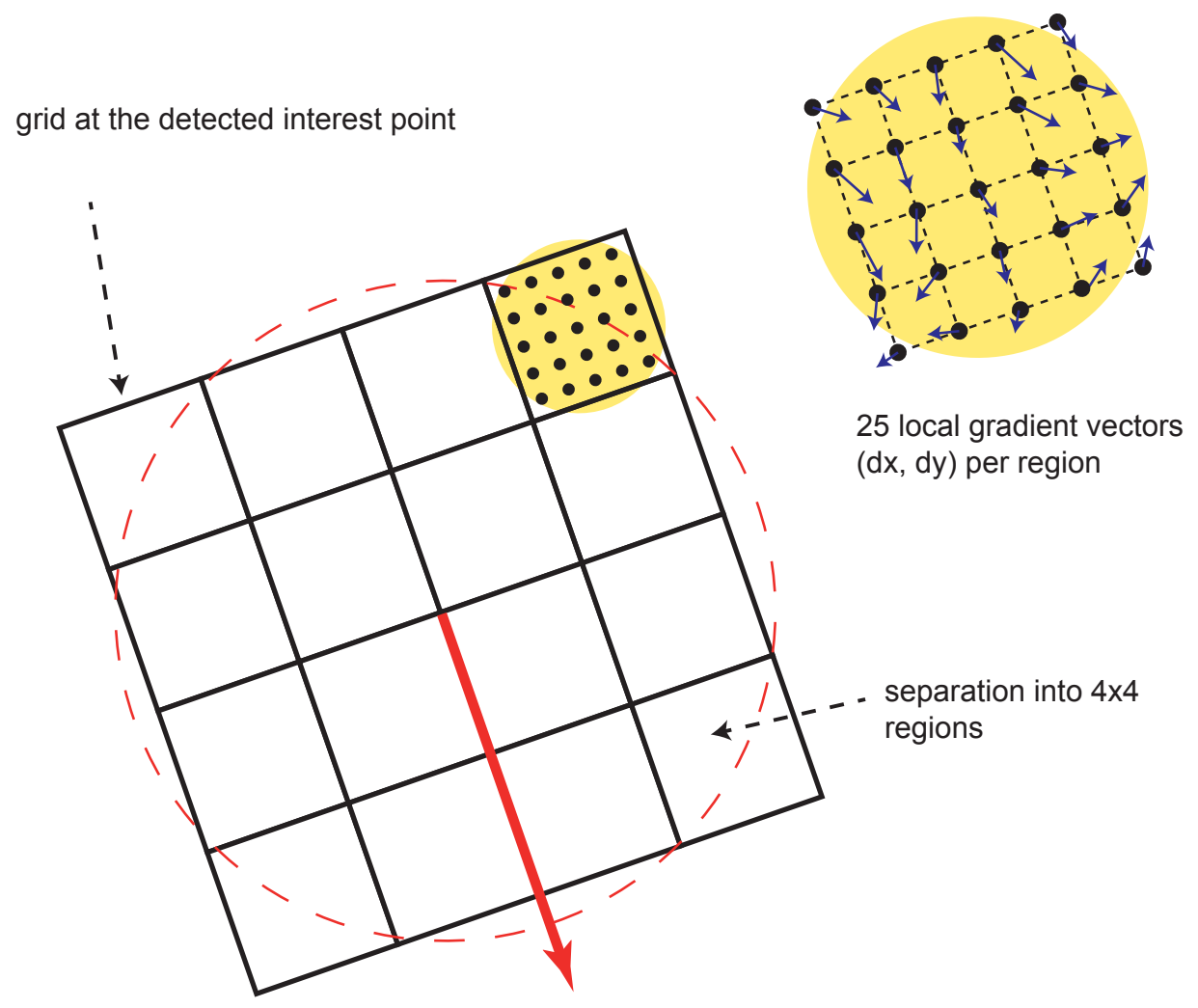

Figure 2.5: Schematic illustration of the SURF descriptor computation adopted from [BETG08].

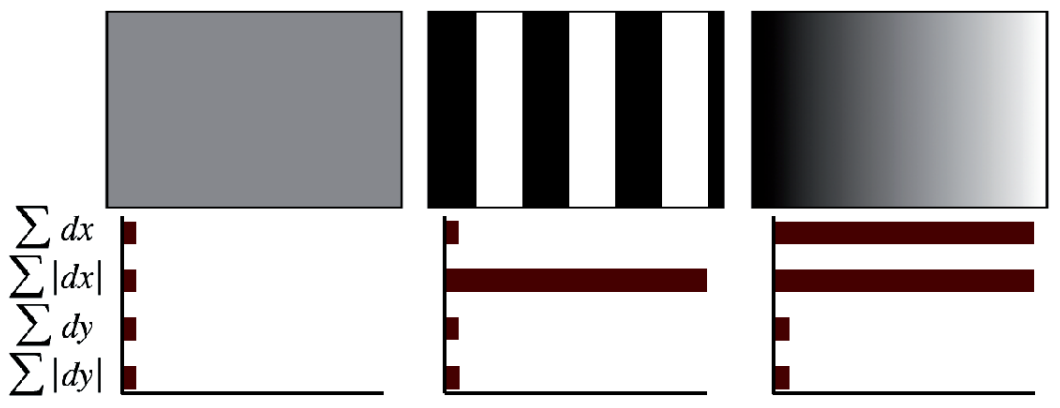

Figure 2.6: Descriptor entries of a SURF descriptor sub-region for three different image intensity distributions. Image reproduced from [BETG08].

entries is weighted with a Gaussian distribution centered in the middle of each subregion indicated by a yellow circle in Figure 2.5. This reduces border effects caused by small errors in the interest point location. In general, the descriptor is designed to be minimally affected by small changes with respect to its location, scale, and orientation, while still maintaining its distinctiveness. To this end the descriptor entries are once more weighted by a Gaus- 
sian distribution centered in the middle of the complete feature patch. Finally the resulting descriptor vector is normalized to length 1 to be invariant against illumination changes.

With SURF being a prominent example, most feature descriptors are high dimensional vectors. To identify a matching feature pair, their similarity is determined by computing the Euclidean distance between the descriptor vectors.

The 128-dimensional scale-invariant feature transform (SIFT) Low04 descriptor, its extension, Gradient Location and Orientation Histogram (GLOH) MS05, and its speeded up 64-dimensional version SURF [BETG08] are considered state-of-the-art. A comprehensive overview is provided in [MS05].

In the case of location recognition, it can be assumed that database as well as query images are recorded upright or rotated by $90^{\circ}$. Hence, in plane rotation invariance of the descriptor is not required. This not only significantly reduces the extraction time on the mobile device but also increases the distinctiveness of the descriptors. To reduce the latency caused by the transmission of feature descriptors from the mobile device to the server, compressed feature descriptors that require only very little memory have been designed. The most prominent representative is the compressed histogram of gradients $(\mathrm{CHoG})$ descriptor $\left[\mathrm{CTC}^{+} 09 \mathrm{a}\right]$ requiring only 60 bits per descriptor whereas SURF requires 8 bits for each of its vector entries.

Throughout the following chapters a combination of MSER and SURF is used due to the fast detection and extraction times, which are critical for mobile applications, and the comparably low dimensionality of the SURF descriptor. This is important as quantization effects increase significantly in high dimensional spaces. The widespread adoption of SURF should allow for a fair comparison of image retrieval approaches.

\subsubsection{Bag-of-Features based Image Retrieval}

With local image features, the similarity between two images is typically determined by the number of matching feature descriptors. In turn, the similarity between features is determined by the Euclidean distance between their descriptors. Thus, when searching for a matching feature in a database of possibly billions of features, we are searching for the nearest neighbor $(\mathrm{NN})$ in the descriptor space. Clearly, this can be a very time consuming task if each vector has to be compared to every other vector (exhaustive search).

In order to avoid that query times scale linearly with the number of descriptors, efficient indexing structures are required. For low-dimensional spaces, search times lower than a full, exhaustive search can be achieved using tree-based indexing structures. The tree structure recursively subdivides the descriptor space into distinct subspaces until a given maximum level is reached. In the case of the so-called kd-trees [FBF77], planes, orthogonal to the axes of the descriptor space, are defined to split the database descriptors into two approximately equally sized subsets (see Figure 2.7). After a few subdivisions, the leaves of the tree structure (forming the last level and containing the smallest subspaces) include a small number of feature descriptors each.

When searching for the nearest neighbor $(\mathrm{NN})$ of a given query feature, we only have to traverse the tree structure to identify the corresponding leave and thus the NN candidates that are contained within it. Thus, instead of an exhaustive search, we only have to evaluate on which side of the decision plane the query feature is lying to recursively identify the corresponding subspaces. Once the NN candidates have been found, the NN is identified by 

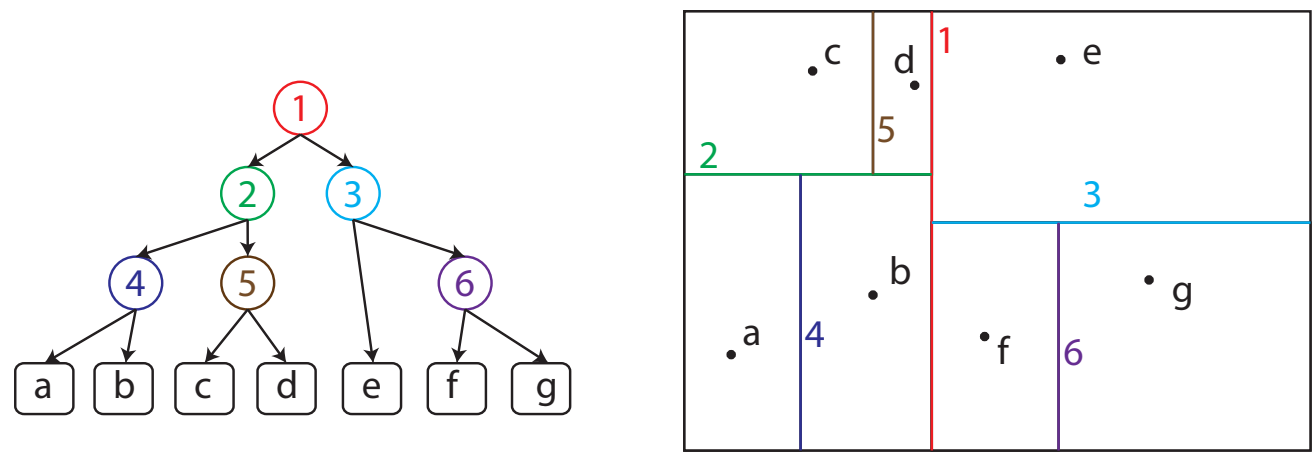

Figure 2.7: Example of a kd-tree structure in a two dimensional space. Each plane, indicated by their respective number, separates the set of data points, indicated by a letter, into two approximately equally sized subsets. After a few subdivisions, every leave node consists of only one data point each (in this example). A nearest neighbor (NN) to a new data point is found by traversing the tree structure to identify the corresponding leave and thus the $N N$ candidate(s).

applying an exhaustive search among them.

However, in multidimensional descriptor spaces, a subdivision using (hyper-)planes is only an approximation. There might be descriptor vectors closer to the query features in spatially neighboring subspaces. Thus, the search has to be unwound to inspect other paths of the quantization structure that might lead to leaves with even closer data points. For low dimensional data, this backtracking effort is relatively low, but as the number of dimensions increases, as a consequence of the curse of dimensionality, more and more alternate paths have to be considered. As a rule of thumb, the number of data points in the reference database should be much greater than $2^{D}$ in order to achieve a speedup compared to exhaustive search, where $D$ is the number of dimensions [JEGE04].

Beyond 10 dimensions, sub-linear complexity can only be achieved with approximate NN search algorithms. Here, it is no longer guaranteed that the true nearest neighbor is found. Rather a probability of typically $95 \%$ that the returned $\mathrm{NN}$ is the true $\mathrm{NN}$ is provided. A very popular algorithm is the so-called Best Bin First (BBF) algorithm [BL97]. It achieves sub-linear search time by stopping the search once a certain number of leaf nodes have been examined. In the spirit of informed searches, the algorithm keeps a priority queue of promising paths in the tree (i.e., those where the distance between the query and the node's center is small), and inspects the most likely paths first. Although this algorithm is faster than exhaustive search, it still needs to inspect a large number of leaf nodes in order to achieve reasonable retrieval accuracy.

A more advanced approach proposed by [SAH08] builds multiple kd-trees. They differentiate from each other by applying different (randomized) splitting planes. Querying the trees simultaneously using a single priority queue reduces the quantization effects. In [ML09] this so-called forest of randomized kd-trees is shown to perform best on most datasets.

The disadvantages of computing the image similarity based on the number of matching feature descriptors are that first, all descriptors of all database images have to be stored, resulting in enormous memory requirements and second, a time consuming exhaustive search 
is performed at the leaves of the tree. In [LMGY04] it is estimated that $95 \%$ of the time is spent on verifying an already found nearest neighbor.

Sivic and Zisserman reformulate in [SZ03] the image retrieval problem into a text retrieval problem by quantizing the feature descriptors into so-called visual words. A visual word can be understood as a representative of similar feature descriptors and is determined via data clustering approaches. Figure 2.10 shows a schematic tree based clustering approach. Image patches associated to the highlighted blue nodes are illustrated on the right side. With increasing tree level, the subdivision of the descriptor space becomes more and more finegrained. Thus, the variance among image patches associated with a single node is more and more reduced. Ultimately, the patches are recordings of one and the same texture and can be represented by a visual word index.

In [SZ03] the k-means quantizer has been applied to determine those centroids (visual words) in the descriptor space that best describe all detected features in the training dataset. In a first step, $K$ random centroids $\mu_{\mathbf{k}}$ are chosen to initialize the iterative k-means algorithm. Second, the training data is separated into $K$ sets $P_{k}$ by assigning all features $\left(\mathbf{x}_{\mathbf{j}}\right)$ to their closest centroid:

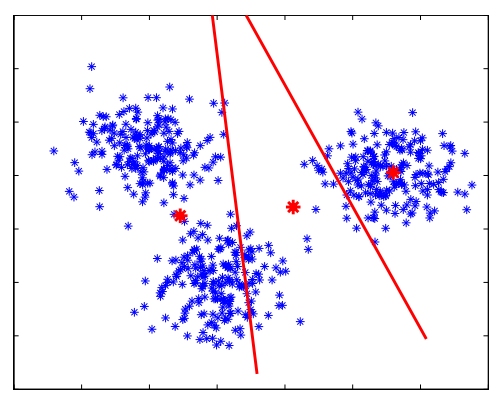

(a)

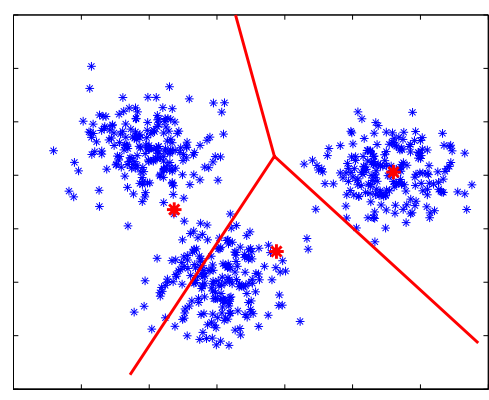

(b)

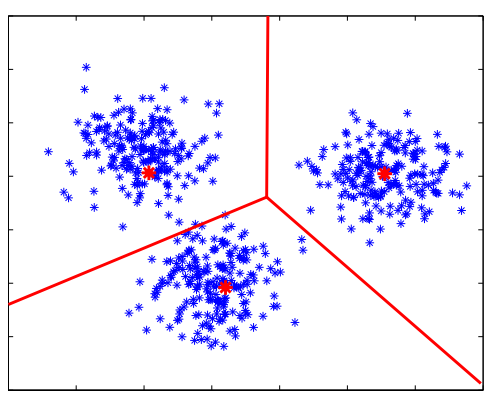

(c)

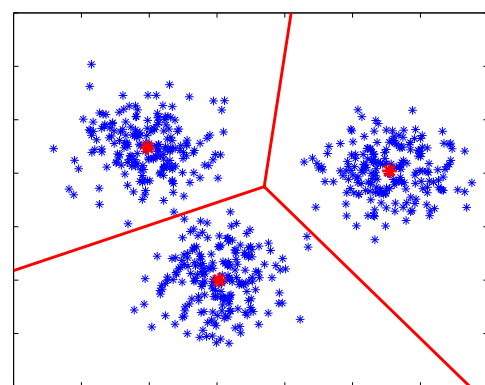

(d)

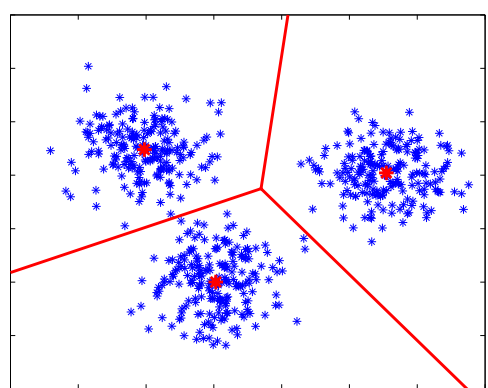

(e)

Figure 2.8: Iteration steps of the $k$-means algorithm. $K=3$ centroids (red stars) are trained to represent the blue data points. The initial step is shown in (a) where random points are selected as centroids. All blue data points are assigned to their closest centroid forming three sets. The means of these three sets define the new centroids shown in (b). This process continues until the positions of the centroids converge (e). Red lines indicate equal distances to the closest centroids and are the borders of the so-called Voronoi cells defining the area covered by a centroid. 


$$
P_{k}=\left\{\mathbf{x}_{\mathbf{j}}:\left\|\mathbf{x}_{\mathbf{j}}-\mu_{\mathbf{k}}\right\|^{2}<\left\|\mathbf{x}_{\mathbf{j}}-\mu_{\mathbf{i}}\right\|^{2}\right\} ; i=1 \ldots K, i \neq k
$$

Third, based on the subsets $P_{k}$ new centroids are computed:

$$
\mu_{\mathbf{k}}=\frac{1}{\left|P_{k}\right|} \sum_{\mathbf{x}_{\mathbf{j}} \in P_{k}} \mathbf{x}_{\mathbf{j}}
$$

This process iterates until the mean distance between the centroids and their set members does no longer decrease. Hence, at fine quantization, descriptors associated to a centroid (visual word) follow a texture pattern that is sufficiently represented by their mean. In Figure 2.8 exemplary iteration steps of the k-means algorithm with $K=3$ centroids are illustrated.

Thus, an image is no longer represented by its descriptors but by a set of visual words. As the same visual word can appear multiple times within a single image, e.g., on repetitive structures like windows, a visual word frequency histogram, the so called Bag-of-Features $(\mathrm{BoF})$ vector is formed. Whereas, certain visual words appear in almost all images, some are very rare and are a strong indication for a particular image. To account for this, each entry in the BoF vector is weighted with the so-called Inverse Document Frequency $w_{k}$.

$$
w_{k}=\log \left(\frac{N_{\text {total }}}{N_{\text {node }}}\right)
$$

The Inverse Document Frequency (IDF) is defined as the log-ratio between $N_{\text {total }}$, the total number of images and $N_{\text {node }}$, the number of images that contain the visual word (see Equation 2.4). In compliance to stop lists in text retrieval approaches, very frequent visual words are suppressed. Hence each entry in the BoF vector is formed by the multiplication of the Term Frequency (TF), which indicates the number of occurrences of a visual word in a particular image, and Inverse Document Frequency (IDF). The overall process of generating a BoF vector, including feature extraction and feature quantization, is summarized in Figure 2.9

The similarity $s$ of any two images can be defined by any $L_{p}$-distance between the normalized BoF vectors.

$$
s=\|\mathbf{q}-\mathbf{d}\|_{p}
$$

Normalizing the BoF vectors to length 1 is important in order not to discriminate images with a large or small number of features. In Equation $2.5 \mathbf{q}$ and $\mathbf{d}$ correspond to the normalized query and database BoF vectors, respectively. As a visual vocabulary comprises several hundred thousand visual words and typically, an image is represented by at most a few hundred visual words, the BoF vectors are very sparse. This property can be exploited by computing the distance between the query and all database vectors via so-called inverted files [WMB99]. A simple example of an inverted file is a book index, which is used to identify those pages that include a given word, avoiding the tedious task of going through every single page. When searching for a specific text line, we look up the pages of several words included in the text line and build the intersection. Similarly, each visual word references all images that include it. Figure 2.10 illustrates the association of database images to visual words via 


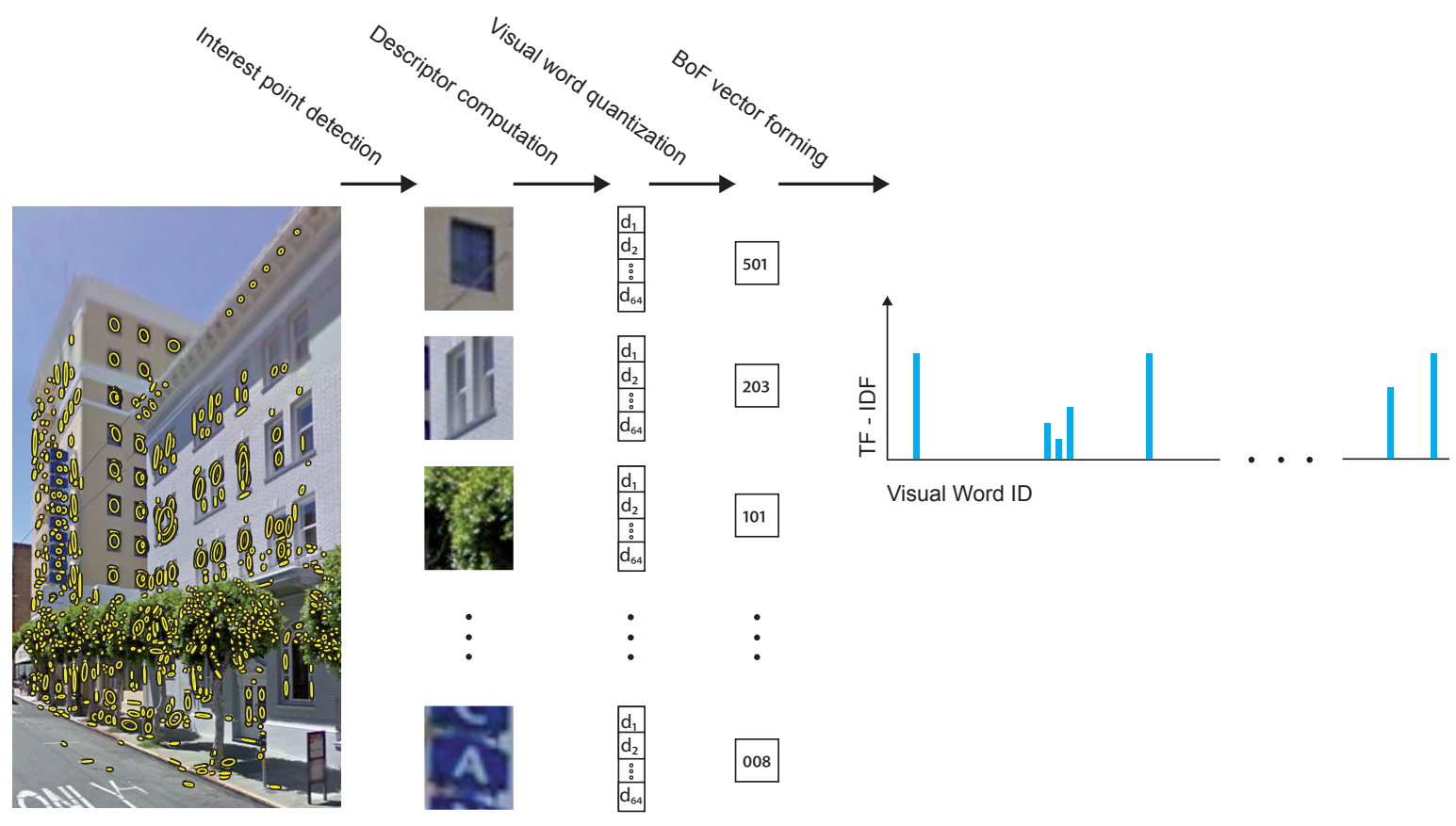

Figure 2.9: Summary of the Bag-of-Feature vector computation. Interest points / regions are detected in an image and the corresponding texture is robustly summarized in a feature descriptor vector (see Section 2.2.1 for details). Descriptor vectors are quantized into representatives termed visual words via, e.g., k-means clustering. Ultimately the BoF vector is formed as a visual word frequency histogram where the entries are weighted in accordance to their Inverse Document Frequency)

an inverted file structure (here the visual words are the leaves of the tree based quantizer). At each visual word all database images are referenced that include this particular word. Thus, to identify the visually most similar images we only have to consider those images that are referenced at the visual words contained in the query image. This can be formalized by accessing only the non-zero entries of the BoF vectors when incrementally computing their distances. In the case of the $L_{2}$-distance, the sum can be subdivided as follows [NS06]:

$$
s^{2}=\|\mathbf{q}-\mathbf{d}\|_{2}^{2}=\sum_{i}\left(q_{i}-d_{i}\right)^{2}=\sum_{i} q_{i}^{2}+\sum_{i} d_{i}^{2}-2 \cdot \sum_{i} q_{i} \cdot d_{i}
$$

To simplify the equations, the square of the similarity $s$ is used in the case of the $L_{2}$-norm. As both vectors are normalized to length 1, this results in:

$$
s^{2}=2-2 \cdot \sum_{i} q_{i} \cdot d_{i}
$$

Thus, the sum has to be evaluated only for those visual words that occur in the query image, as for all other visual words, $q_{i}$ and hence $q_{i} \cdot d_{i}=0$. Likewise, the sum does not contribute to the score of database images that do not comprise the visual word $i$. However, for other norms, such as $L_{1}$, the expression inside the sum cannot be expanded: 

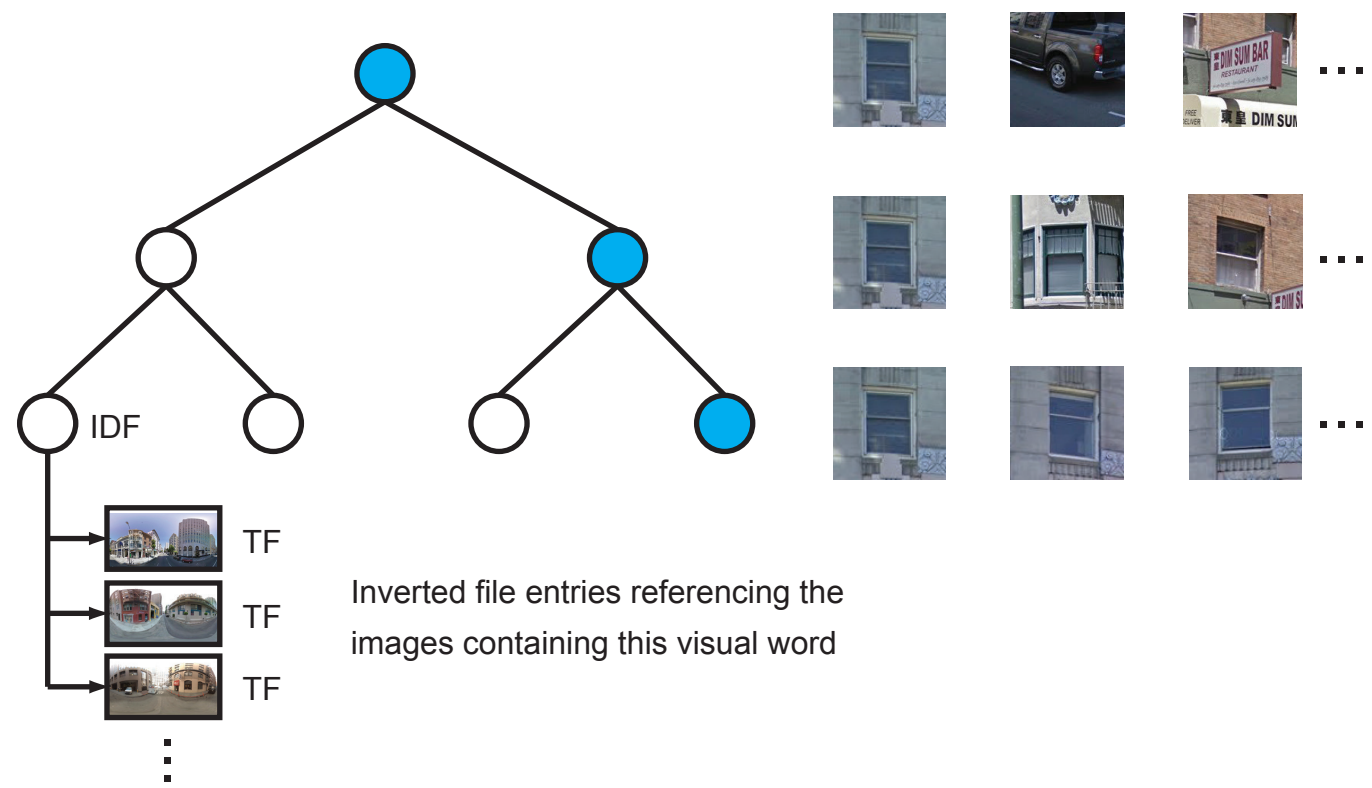

Figure 2.10: Illustration of a tree based BoF quantization and indexing structure. Image patches assigned to a single node decrease in diversity with increasing descriptor quantization. The leaf nodes are defined as visual words with associated inverted files. These reference the images to be scored when a query image patch is quantized to the respective visual word. (Reproduced from [SHC 11$]$, (c) 2011 IEEE)

$$
s^{p}=\|\mathbf{q}-\mathbf{d}\|_{p}^{p}=\sum_{i}\left|q_{i}-d_{i}\right|^{p}
$$

Again the equations are simplified by computing $s^{p}$. Splitting the sum into parts where either the query, the database, or neither of the entries are zero yields:

$$
s^{p}=\sum_{i \mid d_{i}=0}\left|q_{i}\right|^{p}+\sum_{i \mid q_{i}=0}\left|d_{i}\right|^{p}+\sum_{i \mid q_{i} \neq 0 \wedge d_{i} \neq 0}\left|q_{i}-d_{i}\right|^{p}
$$

This expression cannot be calculated using an inverted file scheme as all BoF entries need to be compared. Using the fact that $\mathbf{q}$ and $\mathbf{d}$ are normalized allows us to rewrite the first two parts by the subtraction between the norm and the sum of $q_{i}$ respectively $d_{i}$ at non-zero entries. This results in an expression which can be evaluated using inverted files:

$$
\begin{aligned}
s^{p} & =2-\sum_{i \mid d_{i} \neq 0}\left|q_{i}\right|^{p}-\sum_{i \mid q_{i} \neq 0}\left|d_{i}\right|^{p}+\sum_{i \mid q_{i} \neq 0 \wedge d_{i}(I) \neq 0}\left|q_{i}-d_{i}\right|^{p} \\
& =2-\sum_{i \mid q_{i} \neq 0 \wedge d_{i} \neq 0}\left(\left|q_{i}\right|^{p}+\left|d_{i}\right|^{p}-\left|q_{i}-d_{i}\right|^{p}\right)
\end{aligned}
$$

Figure 2.11 outlines the basic modules of a BoF-based image retrieval system. As a first step after the feature extraction, the quantization structure is trained and the inverted files 


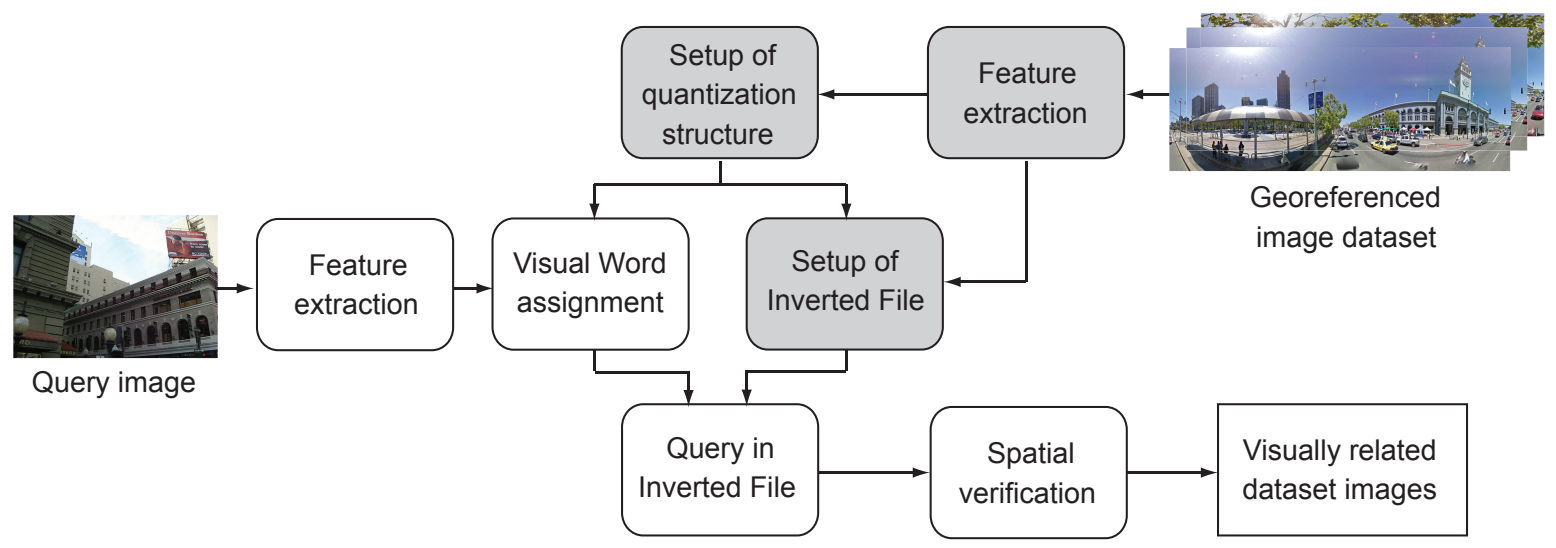

Figure 2.11: Illustration of a BoF-based image retrieval system. The gray modules have to be performed only once to setup (train) the system.

are generated (grey blocks in Figure 2.11). This process can be time consuming but has to be performed only once for a given dataset. The white blocks are part of the query process, which is intended to be performed close to real-time. For now, we can assume that query features are extracted on the mobile device and optionally compressed as described in Section 2.2.1. Using a quantization structure, query descriptors are assigned to visual words and a BoF vector is formed. The inverted file structure allows for a fast computation of the similarity score of database images, which are subsequently ranked accordingly. As the BoF representation ignores the geometric layout of the features within the image, exploiting the spatial relation among them can improve the retrieval performance. Recently, several approaches have been proposed, which either post-process a subset of the retrieved images to verify and rerank the results or integrate the information into the retrieval step [SZ03, $\mathrm{PCI}^{+} 07$, JDS10, WKIS09, LSP06, OWAB07. This optional spatial verification and reranking step concludes the image retrieval process. The major advantages of BoF based image retrieval are its compactness, the reduced storage requirements, and the low query times. This is achieved by the use of inverted files and the avoidance of backtracking.

Despite the analogy to text retrieval approaches, there are some crucial differences. First, in text retrieval all words of the query should be found. However, only a portion of the visual words is expected to be retrieved due to occlusions and imperfect interest point detector repeatability. Whereas textual words can be unambiguously associated to their stems, the variance of the descriptors extracted from a corresponding texture can be large and significantly overlap with descriptor clusters of different textures. Due to the scale, lighting, and affine invariant descriptor extraction, the distribution of matching features is difficult to estimate and is unique for each texture.

\subsubsection{Visual Word Quantization and Indexing}

The assignment of high dimensional feature descriptors to visual words is a challenging task, which is performed with quantization structures like the mentioned k-means based quantizer. Whereas the assignment to a visual word allows for efficient retrieval architectures, the quantization itself reduces the distinctiveness of a descriptor by abstracting them as one 
word. Choosing the coarseness of quantization is a tradeoff between discriminative power and false association due to the mentioned descriptor variances. This is particularly severe in high dimensional spaces, as the number of adjacent quantization cells escalates with the dimensionality. Thus, the probability of assigning two matching descriptors to the same cell is significantly reduced. Further, the time required to determine the cluster centers and the complexity of assigning a query descriptor increases with the size of the vocabularies.

In the case of large scale datasets, where we search for the most similar images among up to $1 \mathrm{M}$ images, the number of features amounts to about $500 \mathrm{M}$. Good retrieval performances in these datasets require between $200 \mathrm{k}$ and $1 \mathrm{M}$ visual words to sufficiently represent each feature descriptor. If we consider applying the iterative k-means algorithm to the problem of determining the best $1 \mathrm{M}$ centroids to $500 \mathrm{M}$ features, the resulting computational complexity is prohibitive. For every visual word in the visual vocabulary the distance to all feature descriptors needs to be computed at every iteration.

To circumvent this problem, Nistér and Stewénius [NS06 propose the use of a hierarchical k-means quantizer (HKM). Similar to the tree-based quantizer in Figure 2.10, the set of features is subdivided into smaller subsets at each level. With the HKM only a small number of centroids, i.e., $K=3$ in Figure 2.12 , is determined via k-means to split the features into subsets. Within these subsets again $K$ centroids are determined to further refine the features. This process is recursively iterated until a certain depth of the resulting quantization tree is reached. Hence, only a small number of features is assigned to the leaves of this k-means based quantization tree. With $K=10$ a vocabulary size of $1 \mathrm{M}$ can be achieved with a tree depth of $L=6$. Thus, not only can we train large vocabularies on extensive datasets but also the quantization of a query feature to its closest visual word can be performed with just $K \times L$ computations. This allows for increasing distinctiveness while significantly reducing the query time. Further, Nistér and Stewénius propose to not only define the leaves but also inner tree nodes of the quantization structure as visual words. As the parent node of, e.g., a leave, contains $K$-times more features, it can be perceived as an umbrella term for the subjacent visual words. Thus, exploiting the different levels of this tree-based quantizer allows us to improve the tradeoff between distinctiveness (fine quantization) and correct assignment to a visual word (rough quantization).

While the HKM provides many benefits when applied to large scale dataset, the resulting leave centroids are suboptimal representatives of the overall set of features. While k-means is designed to minimize the mean distance of all centroids to all feature vectors, the recursive HKM optimizes this cost only within each subset of its tree-based structure.

Thus, Philbin et al. [ $\mathrm{PCI}^{+} 07$ ] propose to use nonhierarchical k-means clustering, which results in minimal total distortion. To cope with the associated enormous computational complexity, they apply an approximate nearest neighbor (ANN) search algorithm to determine the closest cluster for each feature at each k-means iteration. Philbin et al. suggest in [ $\mathrm{PCI}^{+} 07$ ] to use the previously mentioned forest of kd-trees [SAH08] as an ANN algorithm, where multiple kd-trees are combined with a single priority queue. However, ANN search approaches do not guarantee to return the true nearest neighbor and thus incorrectly assigned features will occur. Nevertheless, with the vast amount of dataset features, the chances are low that a single incorrectly assigned feature will significantly alter the position of the corresponding centroid. The comparison on the recognition benchmark of [NS06] suggests that this approximate k-means algorithm (AKM) outperforms HKM. However, as the query descriptors have to be assigned via approximate nearest neighbor (ANN) strategies, which require 

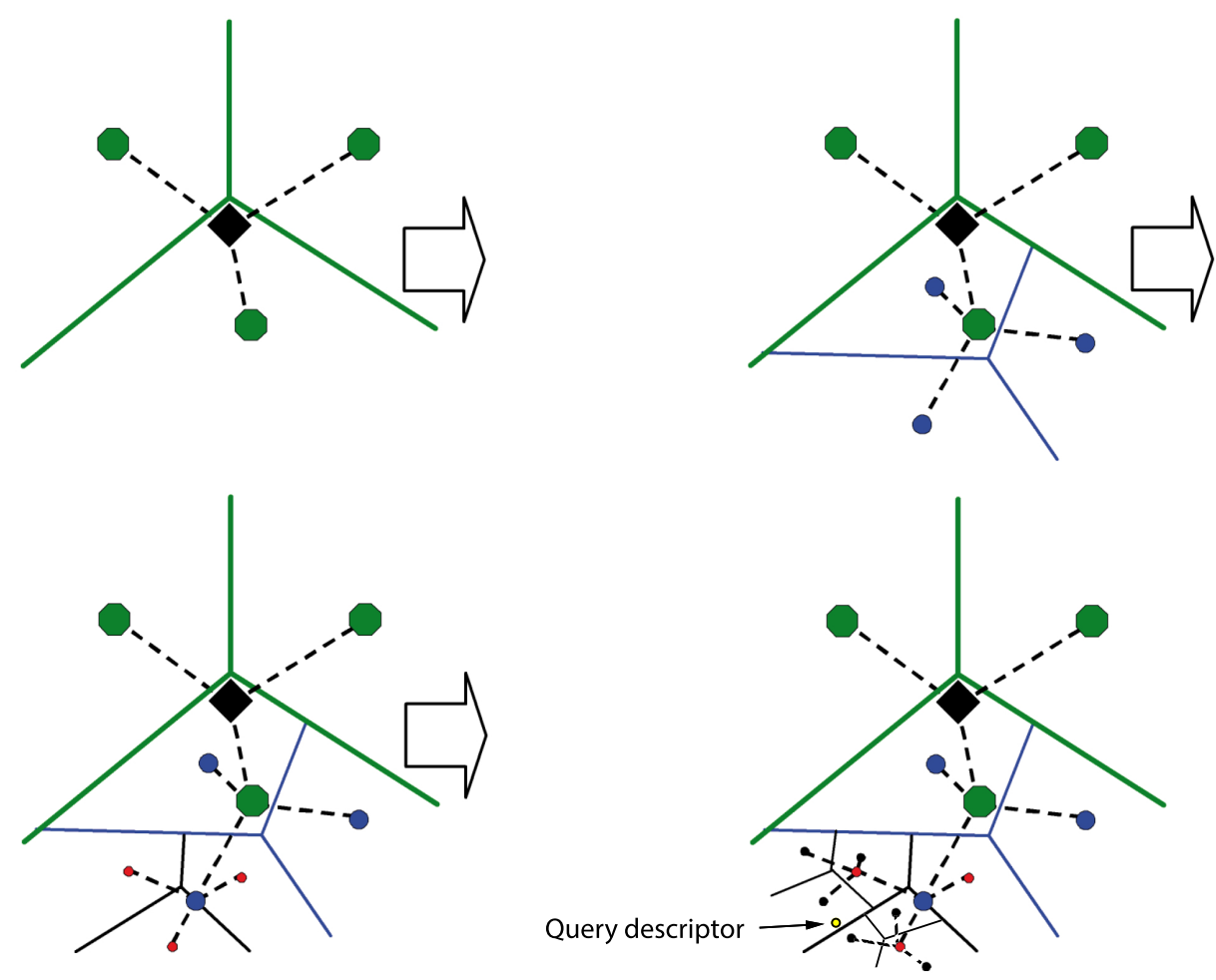

Figure 2.12: Schematic illustration of the individual training stages of the hierarchical $k$ means quantizer (HKM). Colored dots are trained centroids, the edges indicate the borders of the corresponding Voronoi cells. Image reproduced from [NS06].

time consuming backtracking, the increased performance comes at the cost of significantly increased query time.

To achieve the required low query times in location recognition, Schindler et al. SSBS07. approach the quantization problem by considering multiple branches at each traversal step through the HKM-tree (termed Greedy Search). Hence, to identify the closest visual word we do not only follow a single path through the tree. Instead, at every level of the tree, the path is split-up into the $N$ closest centroids requiring an overall of $k+k N(L-1)$ computations. Finally, among all reached leaves the closest centroid is selected. The goal of this approach is to mitigate the negative effects of a tree-based quantizer in high-dimensional space. This problem, however, can even be illustrated in the two dimensional example of Figure 2.12. In the standard HKM quantizer, the query descriptor is assigned to a leave centroid by successively determining the closest centroid at each level of the tree. Finally, the leave centroid at its upper left is selected as its corresponding visual word. However, an even closer leave centroid can be found within a different child node at its lower right. Clearly, with increasing dimensionality these border effects increase dramatically. The proposed greedy search is a remedy, which significantly increases the computational costs as it considers multiple paths through the tree. This allows us to select the closest among multiple leave centroid candidates. Evaluating the performance gain with respect to the number of comparisons per query descriptor shows that an increase of the branching factor $k$ in a standard HKM quantizer achieves similar results. However, while the branching factor cannot be adapted based on the 
currently available time, the number of tree branches considered is flexible.

Jégou et al. [JDS10 follow a different approach by performing a so-called Hamming Embedding of the feature descriptors to allow for a differentiation within a visual word. First, a comparably coarse vocabulary of $20 \mathrm{k}$ to $200 \mathrm{k}$ words is generated via standard non-hierarchical $\mathrm{k}$-means. Subsequently, the difference vector of the descriptors to their centroid (visual word) is dimensionality reduced by a random projection and quantized to a single bit per dimension. Hence, for each database descriptor there exists not only a visual word id but also a short binary vector indicating the position of the descriptor relative to the centroid of its cluster. A query descriptor is treated in the same way. With this additional information, stored within the binary vector (i.e. Hamming Embedding), the distance, and thus the similarity, between a query and a database descriptor can be estimated more precisely. The score for images is set relative to the estimated distances between their database descriptors and the query descriptors. This scheme can be interpreted as an approximate nearest neighbor search in a dimensionality reduced and strongly quantized space.

Whereas BoF based algorithms allow us to cope with databases of approximately 1 million images, the proposed approach in [JDSP10, which is based on Vectors of Locally Aggregated Descriptors (VLAD), achieves a significant reduction in storage requirements facilitating a retrieval within 10 million images on a regular laptop. However, the search accuracy of BoF based algorithms is not outperformed. Further, it has been found that VLAD does not perform well when matching a regular image to all features extracted from one complete location as the aggregation of descriptors is sensitive to the image section. Since in visual localization the size of the reference databases is limited due to the availability of weak prior knowledge on the location, we employ BoF based approaches to cope with the challenging retrieval task of location recognition.

A fair evaluation and comparison among the described algorithms is achieved by adopting evaluation metrics from information retrieval research. Two evaluation measures are generally accepted in the CBIR community and widely-used: Precision is the percentage of retrieved images (locations) that are relevant to the query. Recall is the percentage of images (locations) in the database relevant to the query, which are retrieved. In location recognition images (locations) are defined as relevant by a radius around the query location, as we search for database images that have (approximately) the same location as the query image. Image retrieval algorithms themselves, however, typically do not return a set of retrieved images but a ranked list. Hence, the number of retrieved images is a parameter which can be arbitrarily chosen based on the desired post-processing steps. To fully evaluate the performance of an image retrieval algorithms the two metrics, precision and recall are plotted in a graph where the number of images considered as retrieved is varied from the count of relevant images to a certain percentage of the overall database, e.g., 5\%. This plot is called a precision-recall graph. In location recognition applications, high maxmimum precision values are of particular importance as post verification steps should be avoided to limit the overall processing time. In addition to these metrics, the computational complexity and memory requirements, which are of particular importance in mobile visual location recognition, have to be considered.

$$
\begin{gathered}
\text { Precision }=\frac{\# \text { Relevant AND Retrieved Images }}{\# \text { Retrieved Images }} \\
\text { Recall }=\frac{\# \text { Relevant AND Retrieved Images }}{\# \text { Relevant Images }}
\end{gathered}
$$


The approaches described above are compared on a georeferenced database extracted from Google Street View of an area of about $4 \mathrm{~km}^{2}$, which consists of $5 \mathrm{k}$ panoramas at a distance of $10 \mathrm{~m}$, each of them composed of 12 rectified images. As a query we use 500 images with a resolution of $800 \times 480$ pixels and an opening angle of $70^{\circ}$. They are placed halfway between the panoramas and their field of view is shifted by $45^{\circ}$ to the left with respect to the direction of the street. For a maximum recall of 1 , all panoramas within a radius of $10 \mathrm{~m}$ around the query location have to be in the set of retrieved images. As the graphs show the average over all query images, recall and precision can take arbitrary values between 0 and 1 . This very challenging scenario allows us to effectively evaluate the properties of the individual approaches.

According to Figure 2.13, the basic HKM quantization with $1 \mathrm{M}$ leaves, branching factor $k=10$ and tree depth $L=6$ seems to be clearly inferior to the other approaches. However, as described above, only $k \times L \mathrm{~L}_{2}$ distance computations are required per query, which renders the approach extremely fast. Applying a Greedy Search [SBS07] at query time significantly boosts the performance while requiring $510 \mathrm{~L}_{2}$ distance computations in this configuration $(k+k N(L-1)$, where $N$ is the number of considered branches). Hamming Embedding (HE) [JDS10 with 64 bit signatures requires only about one third of the computations, however, this comes at the cost of significantly increased memory requirements to store the signatures and the projection matrices. While HE achieves highest performance improvements at smaller tree depths, the overall best result has been achieved with $1 \mathrm{M}$ leaves in our scenario. The AKM approach [PCI $\left.{ }^{+} 07\right]$ is set to perform $768 \mathrm{~L}_{2}$ distance computations as part of the backtracking in a set of 8 randomized kd-trees to query a flat vocabulary of $1 \mathrm{M}$ visual words. While it cannot outperform the Greedy Search in this scenario, its globally optimized vocabulary can cope well with even larger distances between the query and reference images (baselines) as shown in Figure 2.14. Here all panoramas within a radius of $20 \mathrm{~m}$ have to be retrieved.

\section{Soft Assignment}

As indicated in Section 2.2.2, there exists a clear mismatch between the hard visual word assignment and the continuous feature descriptor space. While descriptors extracted from different images but identical physical textures can be generally assumed to be in a vicinity of the descriptor space, the actual distance can vary significantly. Due to the nonlinear descriptor vector computation, estimating the variance is difficult as it depends on the image texture.

An approach to cope with the tradeoff between fine and coarse quantization is the soft assignment of descriptors to visual words. Philbin et al. $\left[\mathrm{PCI}^{+} 08\right.$ propose to assign a descriptor not only to the closest visual word but also to words in its vicinity. The corresponding BoF entries are additionally weighted relative to the distance between the word centers and the descriptor vector according to an exponential distribution. Thus, the chance that a matching query and database descriptor are assigned to one of these visual words is significantly increased, while the costs on the query time are very limited. As described in [vGVSG10, different weighting kernels can be applied to reflect the distance between the descriptor and the cluster center and thus the assignment plausibility. Further, soft assignment at query time can be performed by scoring at the 4 closest visual words, as suggested in [JDS10], which have to be identified via nearest neighbor search. This can be beneficial if the inverted files 


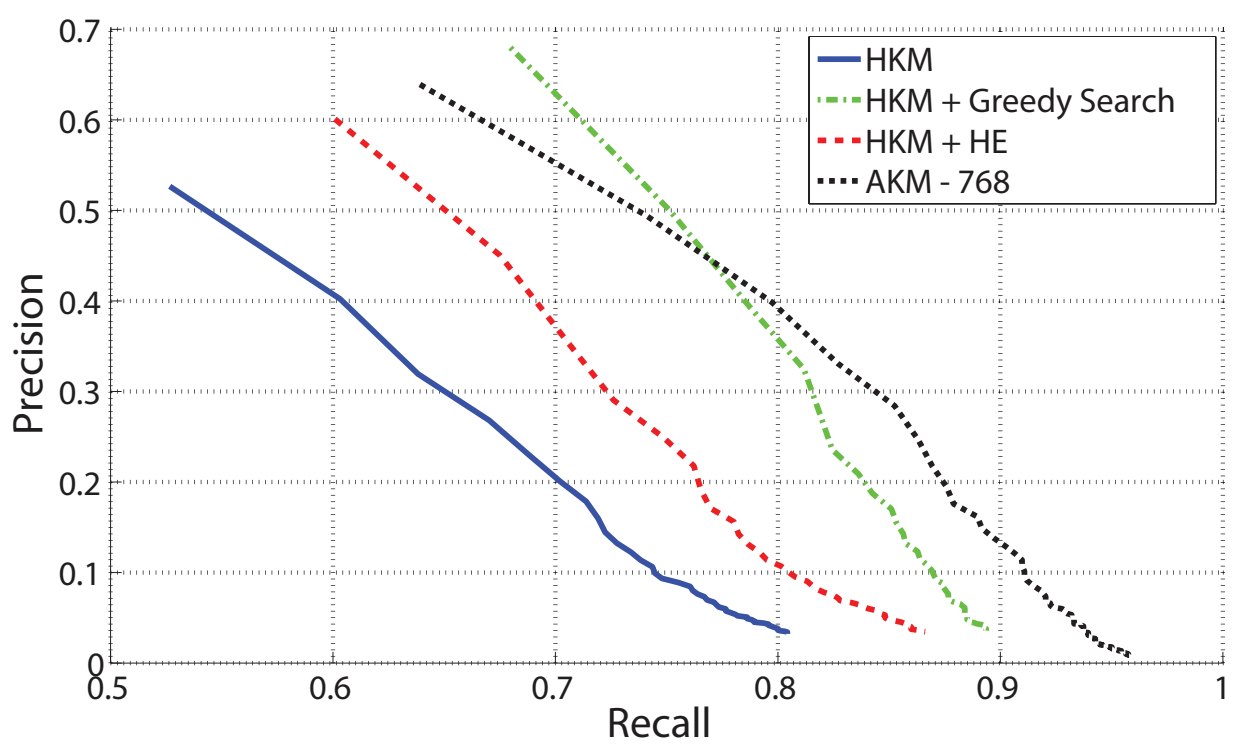

Figure 2.13: Comparison of state-of-the-art quantization and indexing structures based on a Google Street View database. Panoramas within a radius of $10 \mathrm{~m}$ around the query location are considered relevant. (Reproduced from [SHC+11], (C) 2011 IEEE)

memory usage should not be increased.

The hierarchical scoring discussed above can also be interpreted as a form of soft assignment as not only the leaves but also inner nodes are considered as visual words. They vote for all images referenced by their child nodes at reduced weighting (due to their lower IDF). However, since this is only an approximation of the closest word assignment, the performance is inferior.

In Figure 2.14, we apply the soft assignment approach proposed in $\left[\mathrm{PCI}^{+} 08\right]$ to the AKM and the HKM with Greedy Search. The same database and query is used as in Figure 2.13. however, in this experiment all database images within a radius of $20 \mathrm{~m}$ have to be retrieved to push the approaches to their limits. While only a limited increase in performance has been achieved for the AKM, the improvements are much more significant with the HKM. This clearly shows that less optimized vocabularies as those of HKM can be efficiently augmented with the aid of soft assignment.

\section{Spatial Verification}

Representing an image or scene with a BoF vector has been proven to be efficient and distinctive. However, as this abstraction ignores the layout of the features in the image, exploiting the spatial relation among them could significantly improve the retrieval performance. Several approaches have been proposed, which either post process a subset of the retrieved images to verify and rerank the results or integrate the information into the retrieval step [SZ03, $\mathrm{PCI}^{+}$07, JDS10, WKIS09, LSP06, YWY07, OWAB07, TCC ${ }^{+}$10b].

While postprocessing approaches, which allow us to verify feature matches based on the 


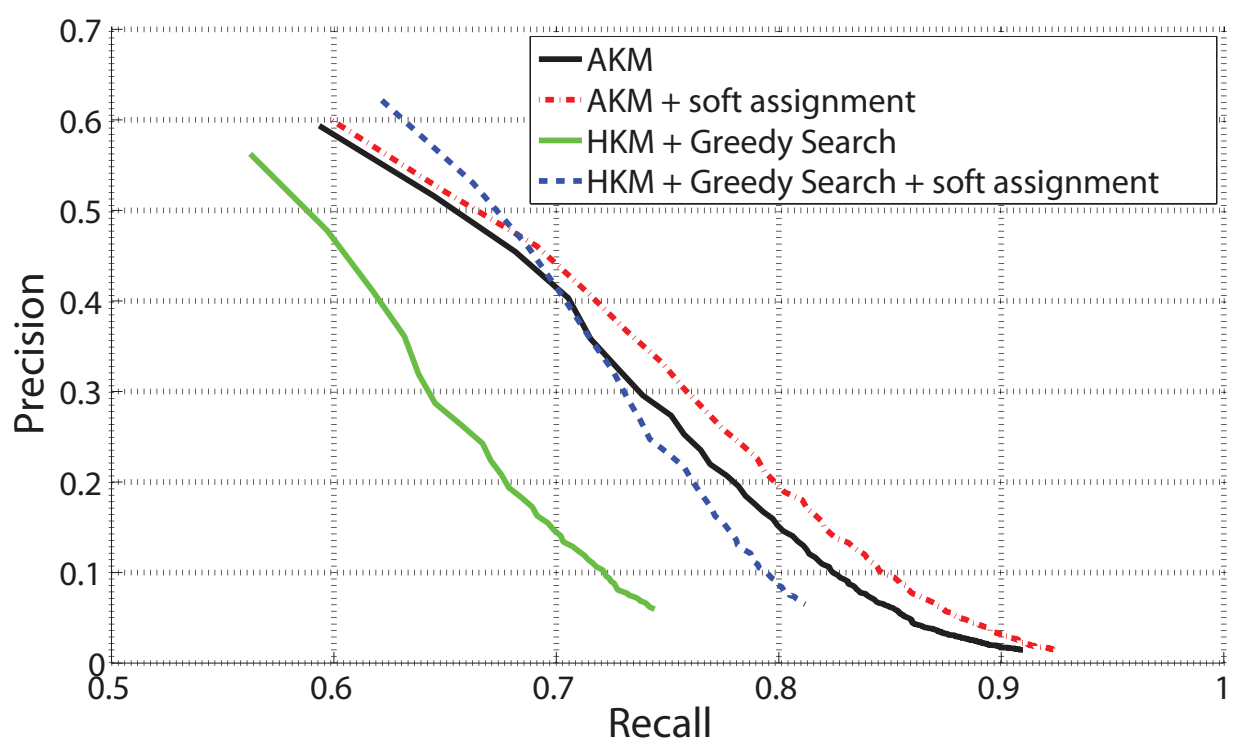

Figure 2.14: Evaluation of soft assignment [PCI+08] applied to AKM and HKM quantization structures. Panoramas within a radius of $20 \mathrm{~m}$ around the query location are considered relevant. (Reproduced from [SHC+11], (C) 2011 IEEE)

epipolar geometry using the RANSAC algorithm [FB81, provide excellent results with respect to the retrieval precision, the computational complexity is very high. In [ $\left.\mathrm{PCI}^{+} 07\right]$ and $\left[\mathrm{CPS}^{+} 07\right.$, the query time is reduced via relaxed geometry models like affine sub-groups of 3 to 5 degrees of freedom, followed by an iterative refinement estimating general affine geometry (6 DOF). Hypotheses are based on single pairs of corresponding features by exploiting the elliptical region shape of affine invariant feature detectors (see Section 2.2.1). As the memory requirements associated with storing the ellipse parameters of every database feature is larger than the inverted file itself, PCM09] proposes to quantize the ellipse by minimizing the average reprojection error. The required 24 bits per feature can be further reduced by assuming that only upright images are recorded in the location recognition application. However, due to the mentioned high computational complexity, the re-ranking of image retrieval results can be applied only to a limited fraction of retrieved database images.

In $\left[\mathrm{CPS}^{+} 07\right.$, a query expansion is suggested, which is a popular strategy in text retrieval systems to increase the recall. Here, the set of query features is expanded by including those found in the already verified retrieval results. However, as in location recognition the relation between the individual database images is known, we require only a single match in the spatial vicinity of the query image.

An appealing approach to incorporate spatial information is to extend the orderless bag of visual words vector by information about the spatial distribution as proposed in [LSP06]. The image is recursively segmented into a pyramid of increasingly smaller sub-regions, and visual word frequency histograms are computed for each of these regions. As the individual histogram vectors are limited to a certain spatial domain, matching can differentiate a good match, where the visual words are arranged in a similar layout as in the query image, from a bad match, where the same visual words are present, but in a different spatial arrangement. 
Similarly, in $\left[\mathrm{CWL}^{+} 10\right]$, ordered BoF vectors are generated by projecting features to multiple one-dimensional subspaces. In the spirit of hash approaches it can be assumed that the majority of ordered $\mathrm{BoF}$ vectors is matching at similar images. However, these approaches tend to be very sensitive to perspective changes at non planar structures, which limits their usefulness for location recognition.

A successful way to increase the distinctiveness of features is to combine different detectors to create feature bundles as described in [WKIS09]. Features are extracted using both an MSER MCUP04 and a SIFT [Low04 detector. All SIFT keypoints within an MSER are aggregated into a feature bundle and described by a bag of visual words vector. This approach groups SIFT features based on the local context provided by the MSER and allows us to enforce local geometry. While being very beneficial for partial duplicate image retrieval, these bundled features are not sufficiently robust against strong perspective distortions.

In OWAB07] co-occurrence information about visual words is incorporated into the retrieval process via the hierarchical k-means quantizer (HKM). For every image in the database, a Boolean co-occurrence matrix is created, where the rows and columns correspond to the visual words in the vocabulary. Given one visual word, which selects a row, the columns selected by its $N$ closest spatial neighbors are set to true to indicate co-occurrence. In order to limit the size of the matrix, the authors use the nodes at a higher level of the HKM quantization tree, which effectively results in a generalization of the visual vocabulary formed by the leaf nodes. They proceed with image retrieval as described in [NS06], and use the similarity between the co-occurrence matrices of the retrieved images and the query image to re-rank the results.

Jégou et al. introduce in JDDS10 a computationally efficient weak geometric consistency (WGC) check that exploits the properties of scale and rotation invariant features. As described in Section 2.2.1, the scale of features is set relative to the size of the objects in the image. Further, a dominant orientation can be determined for every interest region, which provides rotation invariance. Thus, matching two images of the same scene results in a consistent relative scale and rotation for all correct feature correspondences. This consistency check is integrated into the scoring by keeping track of multiple scores for every image, which correspond to different relative scale and orientation hypotheses. The score with the highest number of assigned visual word matches is deemed to correspond to the most likely relative scale and orientation, while other matches are considered to be not consistent. Thus, images are ranked with respect to these maximal scores. While this approach utilizes only two degrees of freedom, it is applied to all relevant images and is shown to be complimentary to the re-ranking approaches described above.

While constraints on the geometry can significantly increase the precision of the retrieval, the described approaches assume at most affine transformations to achieve reasonable performance. However, due to the wide baselines (distances between query and reference images) and complex three-dimensional structures in location recognition, efficient geometric verification and reranking remain a challenge for future research. 


\subsection{Summary}

In this chapter, the two major modules of a visual location recognition system, visual odometry and content based image retrieval (CBIR) are introduced and the state-of-the-art is discussed.

While several novel approaches to the problem of visual odometry have been proposed in the last few years and have been successfully applied to robotic applications, most of them are very susceptible to rapid movements of either the camera or objects in the scene. To limit the computational complexity, visual odometry as well as visual SLAM approaches base on tracking features which quickly fails in the presence of motion blur. Thus, efficient recovery approaches are required that can handle long spatial distances to the last successfully processed frame. Further, visual odometry approaches need to be fused with data from intertial measurement units to first, allow for a more robust tracking and second, to estimate the unknown scale factor in the case of using a singly camera only.

Content based image retrieval can be considered as a very active research area, with significant performance improvements within the last few years. The introduction of robust, affine invariant, distinctive local features like SIFT [Low04] or SURF [BETG08] paved the way for effective image descriptions. The quantization of these feature descriptor vectors to so-called visual words, proposed by Sivic and Zisserman in [SZ03, enables the application of text-retrieval approaches to the problem of image recognition. Several algorithms to perform this complex quantization exist and the most prominent ones are evaluated in this chapter to allow for a fair comparision with respect to the challenging application of visual location recognition. It can be concluded that the approximate k-means (AKM) algorithm allows for the best retrieval performance while the hierarchical k-means (HKM) quantizer achieves the lowest computational complexity. However, as will be discussed in the following chapter, this complexity might be still to high for the application on a mobile device. 


\section{Multiple Hypothesis Vocabulary Tree}

प

Objects recorded at different scale, pose, and background can be distinctively described by BoF vectors with the aid of a robust yet fine quantization of high dimensional descriptors into visual words. In the application to mobile location recognition, typically, only sparse reference images can be assumed. For instance, Google Street View panoramas are available online at a distance of $12.6 \mathrm{~m}$ on average. The three exemplary panoramas in Figure 3.2 illustrate the problem of wide baselines, different lighting conditions, and dynamic objects (panoramas are rectified before feature extraction, as shown in Figure 2.4).

However, most importantly, very low retrieval times are essential due to the rapidly changing field of view of the handheld caused by the constantly changing user attention. Real-time location recognition is an essential prerequisite for most location based services (LBS) and especially for SLAM algorithms [AFDM08]. The retrieval times are governed by the feature extraction on the mobile device, the retrieval of database images on the server, and in particular the delay caused by the transmission of the features from the handheld to the server. To reduce this transmission delay, the approach proposed by Chen et al. $\left[\mathrm{CTC}^{+} 09 \mathrm{~b}\right.$ is applied in this chapter. Instead of features, as illustrated in Figure 1.3, compressed BoF vectors are

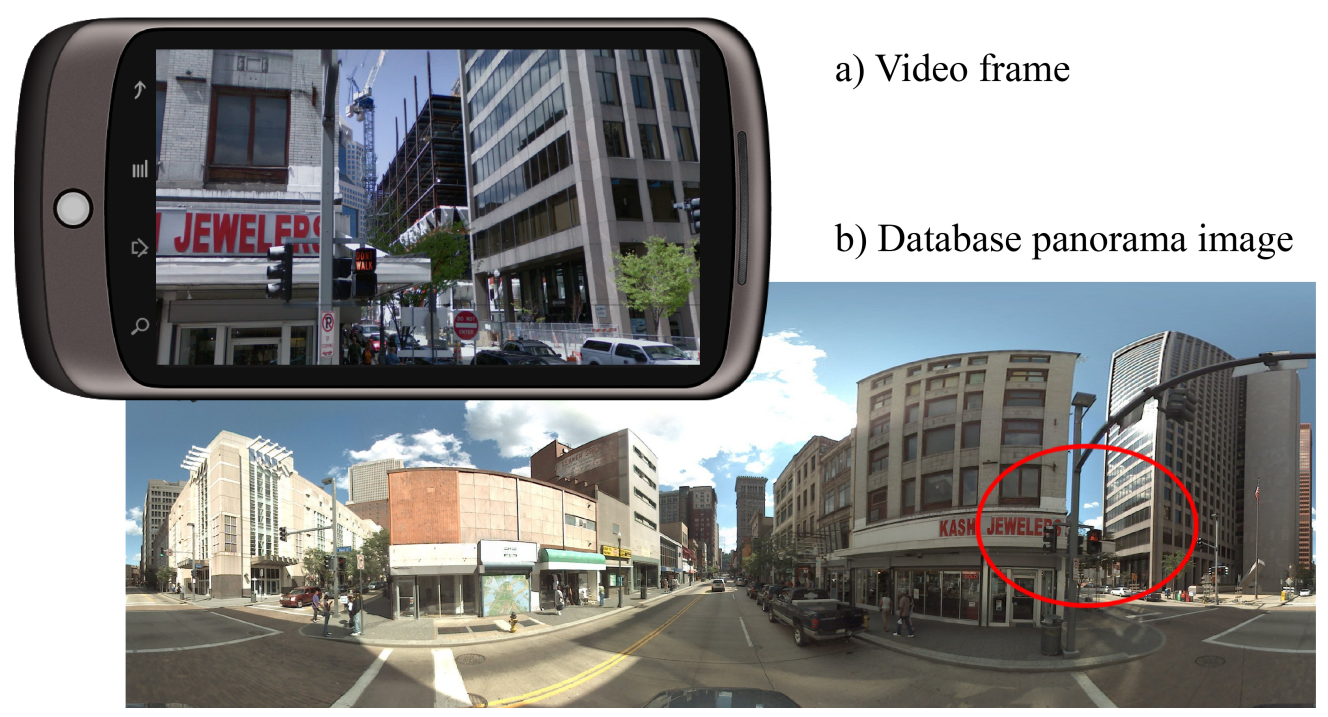

Figure 3.1: Google Street View panorama matched to a low resolution video recording (downtown Pittsburgh) using the Multiple Hypothesis Vocabulary Tree. The red ellipse indicates the parts common to both query frame and retrieved panorama. (Reproduced from [SANH 11$]$, (c) 2011 IEEE)

Parts of this chapter have been published in AN09] and $\mathrm{SANH}^{+} 11$. 


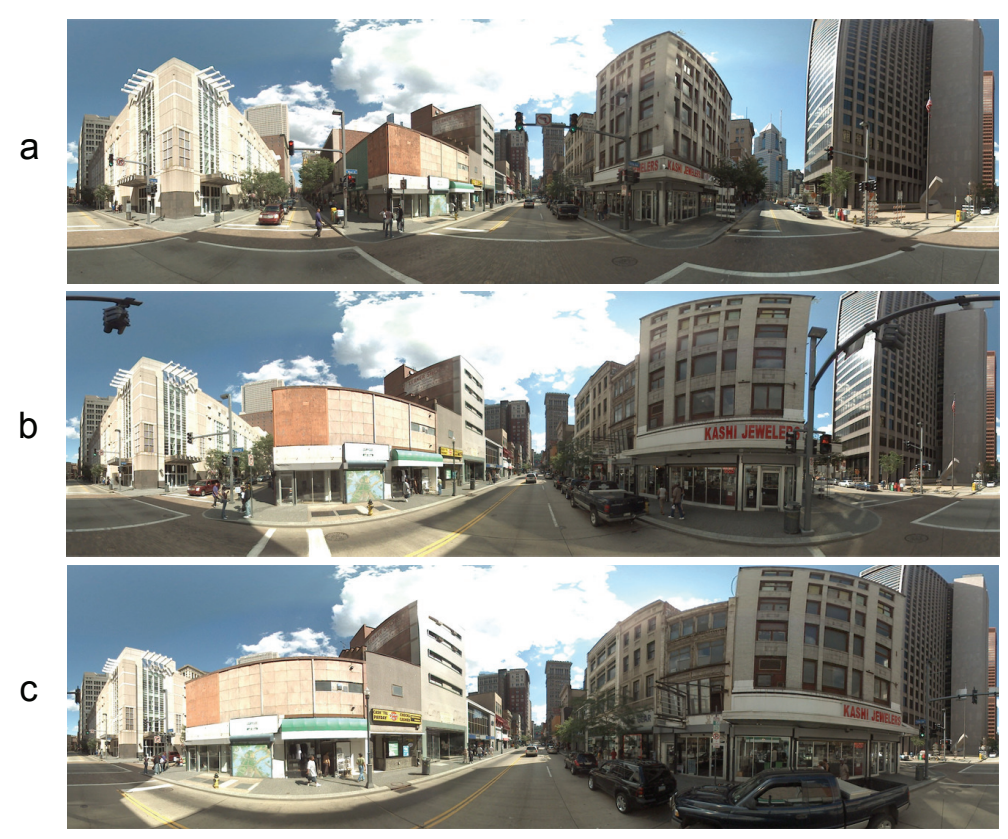

Figure 3.2: Sample images from the Google Street View dataset of Pittsburgh; panoramas are on average 12.6 $m$ apart from each other. (Reproduced from [SANH 11], (C) 2011 IEEE)

transferred to the server. This allows for a more than fivefold rate reduction when compared to compressing features as proposed in [MCCT09] and thus a significant reduction of the overall query time. However, this approach requires performing the quantization of descriptors vectors into visual words on the mobile device at very low complexity to cope with the limited processing power as well as to avoid draining the battery.

To achieve this goal, the Multiple Hypothesis Vocabulary Tree (MHVT) is introduced in this chapter, which allows for the robust quantization of 1000 feature descriptors on a stateof-the-art smart phone (Nexus One with a $1 \mathrm{GHz} \mathrm{CPU}$ ) within $12 \mathrm{~ms}$. Retrieval performance comparable to significantly more complex approaches is accomplished by applying an overlapping buffer at each quantization step, an adaptive clustering approach, and by integrating the probability of correct quantization into the BoF distance calculation.

\subsection{Related Work}

As shown in Figure 3.3 , in which the same dataset as in Figure 2.13 is used for the evaluation, the basic HKM quantization with 1 M leaves, branching factor $k=10$ and tree depth $L=6$ seems to be clearly inferior to the other approaches. However, only $k L=60 \mathrm{~L}_{2}$ distance computations are required per query, rendering the approach extremely fast. Applying a Greedy Search [SBS07] at query time significantly boosts the performance while requiring 510 distance computations in this configuration. Hamming Embedding [JDS10] (HE) requires only about one third of the computations, however, at the cost of increased memory requirements. The AKM [ $\mathrm{PCI}^{+}$08] approach is set to perform 192 distance computations as part of the 


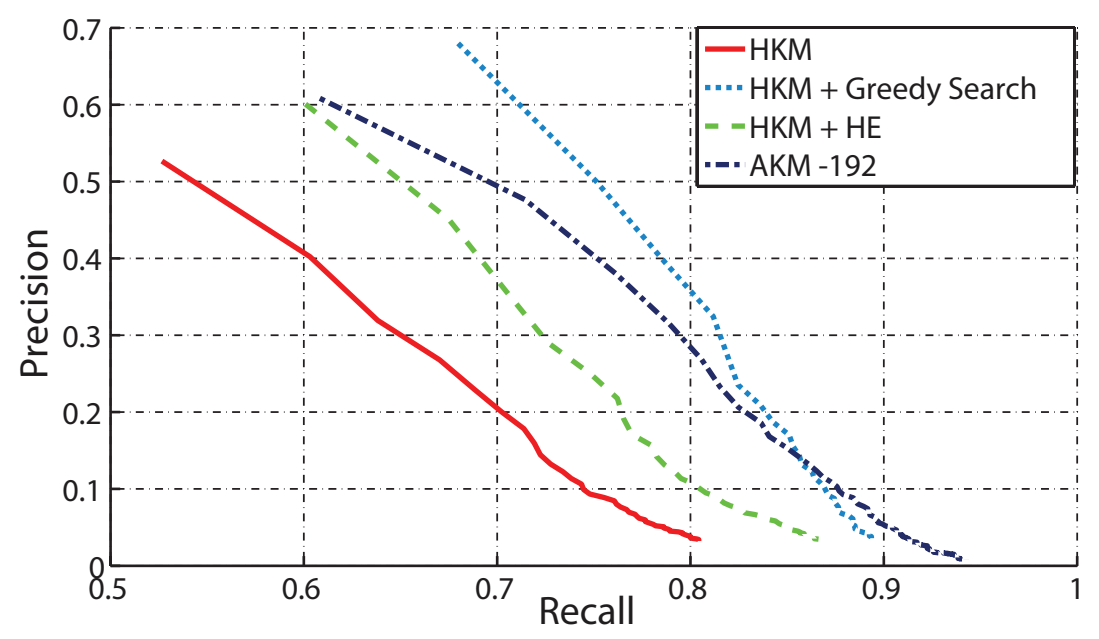

Figure 3.3: Comparison of state-of-the-art quantization and indexing structures based on a Google Street View database. Panoramas within a radius of $10 \mathrm{~m}$ around the query location are considered relevant. (Reproduced from [SANH 11], (C) 2011 IEEE)

backtracking in 8 randomized kd-trees to query a flat vocabulary of $1 \mathrm{M}$ visual words. Soft assignment is applied to the 4 closest visual words. The results of the AKM can be improved by additional backtracking, e.g., using 768 distance computations as in Figure 2.13 results in a $3 \%$ increase in recall, however we try to minimize the query time.

Hence, among the state-of-the-art algorithms, HKM, which requires about 25 ms per query image on a $2.4 \mathrm{GHz}$ desktop $\mathrm{CPU}$ and can be adaptively improved with the aid of greedy search, is most suitable for rapid quantization of descriptors. While this would be sufficient on a regular PC, the limited processing power of mobile devices calls for even faster approaches. To this end, the novel Multiple Hypothesis Vocabulary Tree (MVHT) is introduced as a step towards real-time mobile location recognition.

\subsection{Quantization structure}

Instead of applying a large branching factor $k$, intended to improve quantization in k-means trees [NS06, SBS07, the structure is limited to a binary tree to minimize the query time. Thus, the data is hierarchically organized by separating the space iteratively with hyper planes. At each node, a vector $\tilde{\mathbf{u}}$, heading in the direction of maximum variance, and the median of all data points projected onto $\tilde{\mathbf{u}}$ are determined. Thus, $\tilde{\mathbf{u}} /\|\tilde{\mathbf{u}}\|$ is the normal vector of the hyper plane that separates the node at the median, resulting in two mutually exclusive child nodes. It is essential for the performance of the algorithm that the normal vector is aligned with the direction of maximum variance. While almost optimal results can be obtained by spanning the vector between the two mutually farthest point clusters, the optimal splitting axis can also be determined by the eigenvector corresponding to the largest eigenvalue of the node's covariance matrix. However, in this case the time required to build the tree is increased.

The ratio between the query vector comparisons for a binary tree relative to a tree with 


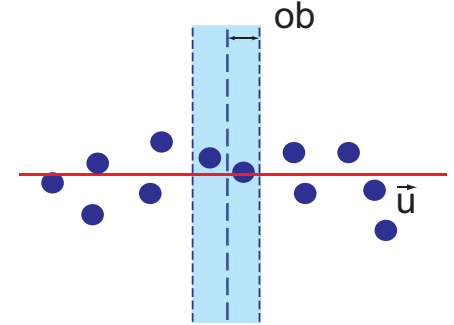

(a) Quantization step 1

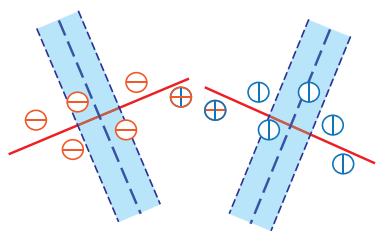

(b) Quantization step 2

Figure 3.4: Quantization with overlapping buffers. (Reproduced from [SANH 11], (C) 2011 IEEE)

branching factor $k$, at corresponding number of leaves, is given by Equation 3.1, where $L$ is the depth of the k-ary tree.

$$
\frac{C o m p_{\text {binary }}}{C o m p_{k-\text { ary }}}=\frac{\log _{2}\left(k^{L}\right)}{k \cdot L}=\frac{\log _{2}(k)}{k}
$$

While for HKM every node has to be represented by a high-dimensional centroid, no separation axes have to be stored for the leaf nodes of a binary tree. In Equation 3.2 the ratio between the number of separation axes $S_{C}$ and the node count $N_{C}$ is given, proving that the binary tree requires at most an equal amount of memory.

$$
\frac{S_{C}}{N_{C}}=\frac{k^{L}-1}{\left(k^{L+1}-1\right) /(k-1)}
$$

A query descriptor is quantized by proceeding down the tree performing high-dimensional dot products with $\tilde{\mathbf{u}}$ to evaluate on which side of the hyper plane it is located. Descriptors close to the splitting boundaries have a high probability of matching to a descriptor in the neighboring node and would require backtracking to be found. Hence, adapted from [LMGY04, an overlapping buffer around the boundary with width $o b=\tau \cdot\|\tilde{\mathbf{u}}\|$ is introduced (see Figure 3.4). All database descriptors projected inside the buffer are assigned to both child nodes. Hence, descriptors that cannot be clearly distinguished by the current decision boundary are not separated from each other at the current node. As the nodes are no longer mutually exclusive, additional quantization steps can be required, which are relative to the parameter $\tau$. However, in practice this is only a minor fraction of the overall number of quantization steps and hardly adds to the query time.

The size of the tree is mainly determined by the aspired quantization level, which can be defined by the number of features assigned to a visual word. Hence, the splitting process described above proceeds recursively until the number of descriptors is less than the defined maximum, the naive count. However, large databases result in differently sized descriptor clusters depending on the frequency of the corresponding image textures (e.g., windows in an urban environment). Thus, using a fixed naive count to stop the quantization is suboptimal. As self-contained feature clusters can be assumed to have similar variance in most directions, the percentage of features inside the overlapping buffer is very high. This allows us to evaluate the separability of a node and to stop the quantization once a certain percentage $\rho$ of features 


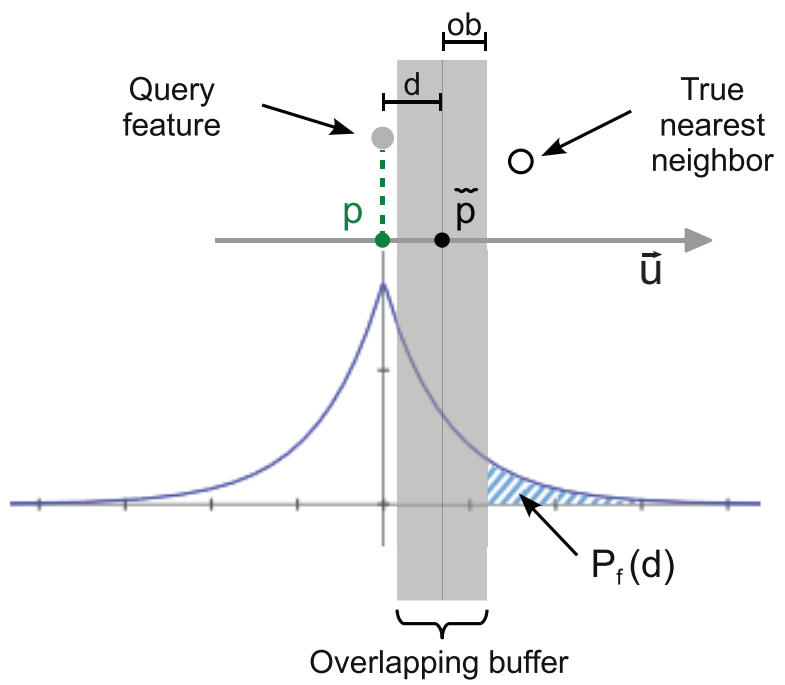

Figure 3.5: Two dimensional illustration of the separation axis $\tilde{\mathbf{u}}$, the overlapping buffer, and the determination of the false quantization probability $P_{f}(d)$ as a function of the distance d between the query feature and the separating hyper plane. (Reproduced from [SANH 11$]$, (c) 2011 IEEE)

fall into the buffer. Thus, over fitting of descriptor clusters can be effectively avoided, resulting in smaller trees with significantly increased performance.

The use of an overlapping buffer to assign descriptors to multiple leaf nodes is comparable to the soft assignment strategies described in $\left[\mathrm{PCI}^{+} 08\right.$. In contrast of assigning database descriptors to all leaf nodes whose centroids are closer than a certain threshold, however, a more sophisticated strategy is applied to increase the probability of assigning both the query as well as the matching database descriptor to the same node. The overlapping buffer ensures that database descriptors follow all hypothetical paths through the quantization tree that a slightly deviating but matching query descriptor could traverse. This allows us to select the most likely hypothetical paths and therefore limits the loss in distinctiveness of the nodes. A hierarchical scoring as applied in [NS06, which can also be interpreted as a weighted multiple assignment of descriptors to leaf nodes, should be no longer necessary. Instead of less distinctive umbrella terms, multiple hypotheses of feature descriptors that correspond to likely word spellings are considered. With the multiple hypothesis approach, the scoring energy can be concentrated on the leaf nodes and extensive time consuming random memory accesses are avoided. We do not assign multiple hypothetical paths to the query descriptor since this would clearly increase the query time.

\subsection{Weighted scoring}

As described above, the probability of assigning a descriptor to the correct child node depends on the distance $d$ between the feature and the separating hyper plane, and the size of the overlapping buffer $o b$ (see Figure 3.5). With the aid of this buffer, quantization effects are reduced. Further, the probability of assigning matching query and database descriptors to 
the same leaf node should be accounted for in the scoring process. Descriptor differences between matching SURF features have been determined to be Laplacian in each dimension, via own extensive empirical evaluation. The mean of this distribution is zero, as it is the difference of two identically distributed random variables. Thus, the probability $P_{f}$ that a matching feature is "incorrectly" quantized to the neighboring child node can be determined by the cumulative 64-dim. Laplacian distribution function. As illustrated in Figure 3.5, this probability corresponds to the integral over the shaded area beyond the overlapping buffer $(o b)$. Thus, it depends on the distance $d$ between the query feature and the separating hyper plane.

We assume that the distribution of the entries of the difference vector between a query and a matching reference descriptor are independently distributed following a Laplacian distribution. Thus, we only have to consider the one dimensional cumulative distribution to determine the probability $P_{f}$ of finding a match on the other side of the buffer (Equation 3.3). Here, $\sigma$ is relative to the variance of the matching descriptor differences $D$.

$$
P_{f}(d)=\frac{1}{2} e^{-\frac{|d+o b|}{\sigma}} ; \quad \sigma=\sqrt{\frac{\operatorname{var}(D)}{2}}
$$

The probability of assigning matching descriptors to the same visual word corresponds to the probability of quantizing matching features to the same node $\left(1-P_{f}\right)$ in all $L$ quantization steps, where $L$ is the depth of the quantization tree (Equation 3.4).

$$
\alpha_{i}=\prod_{m}\left(1-P_{f_{m}}\right)
$$

This probability $\alpha_{i}$ can be utilized to weight the individual comparisons between query (q) and reference (d) BoF vector entries in Equation 3.5, which correspond to the visual words. This allows us to reduce the influence of unconfident visual word quantizations. In this equation, the comparison between a query and a single database image is shown, with the sum iterating over all dimensions of the BoF vector.

$$
\sum_{i} \alpha_{i}\left|q_{i}-d_{i}\right|^{P}=\sum_{i \mid d_{i}=0} \alpha_{i}\left|q_{i}\right|^{P}+\sum_{i \mid q_{i}=0} \alpha_{i}\left|d_{i}\right|^{P}+\sum_{i \mid q_{i} \neq 0 \wedge d_{i} \neq 0} \alpha_{i}\left|q_{i}-d_{i}\right|^{P}
$$

This equation has to be reformulated to allow for a distance calculation via inverted files. As mentioned in Section 2.2.2, only those terms of the BoF vector comparison can be computed efficiently where both $q_{i} \neq 0$ and $d_{i} \neq 0$. The BoF vector entries where either $q_{i}=0$ or $d_{i}=0$ are inaccessible as they are not stored in the inverted file system. Since BoF vectors are normalized to unit length, we can express the sums over inaccessible entries by their complements (see Equation 3.6).

$$
\sum_{i \mid d_{i}=0} \alpha_{i}\left|q_{i}\right|^{P}=\sum_{i} \alpha_{i}\left|q_{i}\right|^{P}-\sum_{i \mid d_{i} \neq 0} \alpha_{i}\left|q_{i}\right|^{P}
$$

Since no weights $\alpha_{i}$ exist for $q_{i}=0$ in the second term of Equation 3.5, we set them to a constant value $c=1$ and perform the same substitution as in Equation 3.6 resulting in Equation 3.7 . 


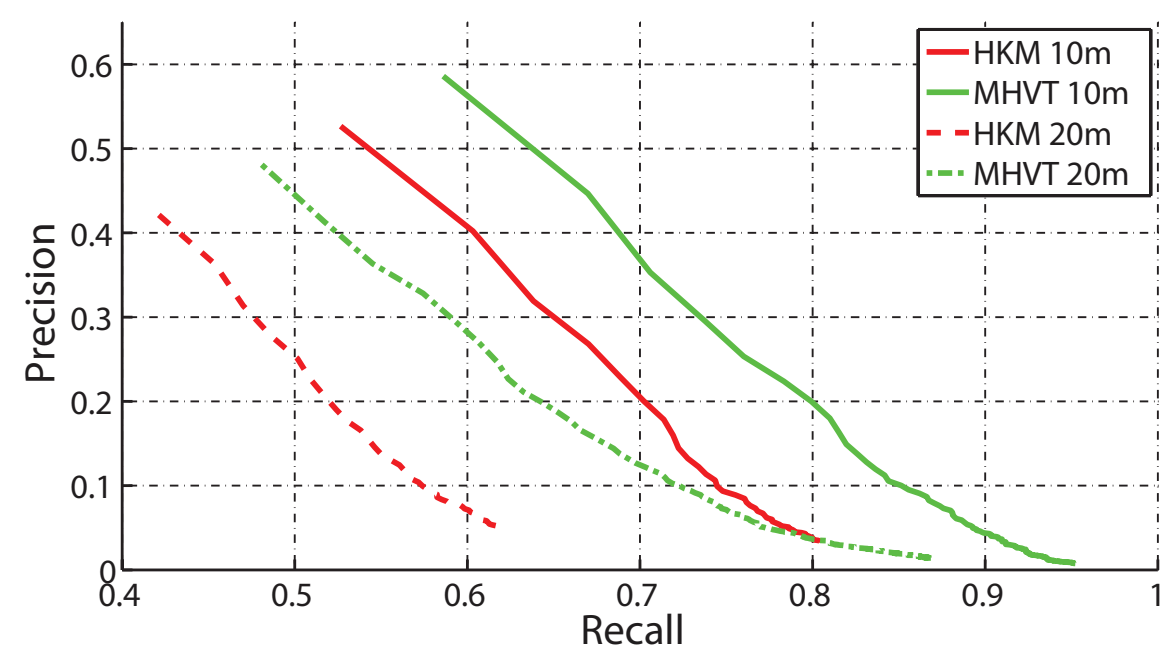

Figure 3.6: Comparison of MHVT with weighted scoring and HKM at two vicinity levels. (Reproduced from [SANH $\left.{ }^{+} 11\right]$, (c) 2011 IEEE)

$$
\sum_{i} \alpha_{i}\left|q_{i}-d_{i}\right|^{P}=\sum_{i} \alpha_{i}\left|q_{i}\right|^{P}-\sum_{i \mid d_{i} \neq 0} \alpha_{i}\left|q_{i}\right|^{P}+\sum_{i} c\left|d_{i}\right|^{P}-\sum_{i \mid q_{i} \neq 0} c\left|d_{i}\right|^{P}+\sum_{i \mid q_{i} \neq 0 \wedge d_{i} \neq 0} \alpha_{i}\left|q_{i}-d_{i}\right|^{P}
$$

Hence, only terms with $q_{i} \neq 0$ and $d_{i} \neq 0$, as well as the weighted norms of the query and the database image, remain in Equation 3.7, which can be computed efficiently within the inverted file approach.

Combining the multiple hypotheses vocabulary with the weighting based on the plausibility of the query descriptor quantization allows us to cope with the continuous feature descriptor space while hardly increasing the query time. In practice, less than 21 high-dimensional vector comparisons have to be performed per query descriptor on average. As time consuming hierarchical scoring techniques [NS06] can be avoided, an overall query time of about $2.5 \mathrm{~ms}$ per 1000 descriptors on a $2.6 \mathrm{GHz}$ single-core CPU using the same database as in [NS06] can be achieved at superior retrieval performance. In Figure 3.6, we compare the HKM approach at $1 \mathrm{M}$ leaves with the MHVT at naive count $=200$ and $\tau=0.06$ using the same database as in Figure 2.13 comprising $24 \mathrm{M}$ descriptors. Locations within $10 \mathrm{~m}$ and $20 \mathrm{~m}$, are to be retrieved, respectively. Larger values of $\tau$ increase retrieval performance further at the cost of higher memory requirements of the inverted file system, which is comparable to the AKM approach with soft-binning.

\subsection{Summary}

To achieve the required low query times, transmission delay is minimized by performing the feature quantization on the mobile device and sending compressed BoF vectors. To cope with the limited processing capabilities of mobile devices, the Multiple Hypothesis Vocabulary Tree is introduced, which allows us to perform the feature quantization at very low complexity. 
A further increase of retrieval performance is accomplished by integrating the probability of correct quantization in the distance calculation. By achieving an at least 10 fold speed up with respect to the state-of-the-art, resulting in $12 \mathrm{~ms}$ for 1000 descriptors on a Nexus One with a $1 \mathrm{GHz}$ CPU, mobile vision based real-time localization becomes feasible. In combination with the feature extraction as proposed in $\left[\mathrm{TCC}^{+} 10 \mathrm{a}\right]$, which takes $27 \mathrm{~ms}$ per frame on a mobile device, extraction and quantization of 500 features can be performed at $30 \mathrm{fps}$. 


\section{System Aspects in Mobile Visual Location Recognition}

口

In the following, the Bag-of-Feature based location recognition system described in Section 2.2 is extended by a high level feature selection and the integration of coarse prior knowledge. Feature selection in both the query image as well as the reference images is an essential prerequisite to allow for a reasonable localization performance under realistic conditions as will be shown in Section 4.3 ,

\subsection{Feature Extraction and Selection}

While local feature detectors like SIFT, SURF, or MSER are designed to find distinctive image patches, they base their decision on the texture rather than high level information like the frequency of a feature in the database. Experiments show that most detectors produce a high number of features on plants (e.g. foliage), which are unreliable due to the constantly changing texture and seasonal changes. Retrieval performance can be significantly improved by excluding those features based on the hue distribution of the corresponding image patch (compare Figures 2.4 and 4.1). Hence, not only the quantization, indexing, and verification but also the selection of features is a major issue in visual location recognition. The selection of the most relevant features allows us to increase the performance and to reduce the amount of data to be transmitted. In the following, appropriate feature extraction and selection approaches are discussed separately for query and database images.

\subsubsection{Query Image}

Important information about the reliability of features can be derived from the query itself. Tracking features across several video frames allows not only for fast feature extraction [TCGP09, DB06]. Based on the time span a feature is successfully tracked, its stability with respect to view point changes can be estimated. Further, moving objects like cars or persons can be detected as the trajectory of associated features is not consistent with the majority of the features. Most importantly, the limited view of a single mobile phone image recording can be extended by generating virtual feature panoramas. These are generated by aggregating all reliably tracked features extracted from images within a short period of time and thus a limited local range. As users tend to pan their camera while recording, a significantly more distinctive query can be composed, which can be considered as a wide-angle recording.

Tracking the features on the mobile device also allows us to send only those which have not been transmitted before and the indices of those that disappeared. This allows for a further

Parts of this chapter have been published in Hui10, AA10 and $\left[\mathrm{SHC}^{+} 11\right.$. 

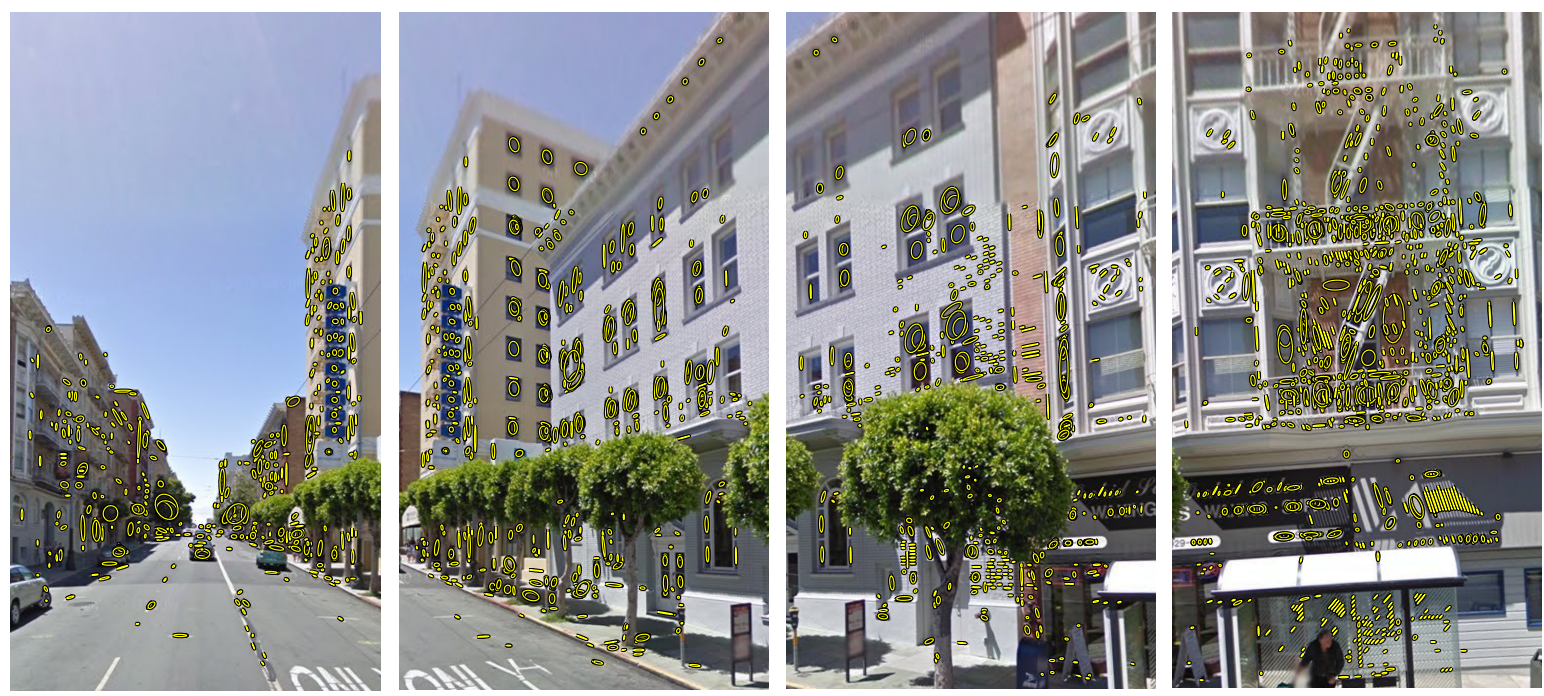

Figure 4.1: Gnomonic projections of a Google Street View Panorama recorded in San Francisco. Ellipses are fitted around affine covariant MSER [MCUP04] regions. Features attached to foliage are successfully detected and removed. (Reproduced from [SHC+11], (c) 2011 IEEE)

significant reduction of the data rate and thus network latency. This concept is also applied to reduce the computational complexity of the BoF based image retrieval. As the image scores are composed of individual feature scores (see Equation 2.11), only those features that have not been assigned to a visual word before need to be quantized. The contribution of an individual feature to the score of database image $i(s(i))$ is shown in Equation 4.1, where $w_{k}$ is the inverse document frequency (IDF), $c_{k i}$ the frequency of the corresponding visual word $k$ in database image $i$, and $\sigma_{i}, \sigma_{q}$ normalization factors for the database and query BoF vectors. As described in Section 2.2.2, the database and query BoF vectors have to be normalized to length 1 . The updated score $s_{\text {new }}(i)$ is composed of the score belonging to the last frame $s_{\text {old }}(i)$ minus the scores caused by disappeared features plus the scores caused by newly assigned features, as shown in Equation 4.2 .

$$
\begin{aligned}
s_{\text {feat }}(i) & =\frac{w_{k}^{2} c_{k i}}{\sigma_{i} \sigma_{q}} \\
s_{\text {new }}(i) & =s_{\text {old }}(i)-\sum_{\text {disappeared }} s_{\text {feat }, d}(i)+\sum_{\text {appeared }} s_{\text {feat }, a}(i)
\end{aligned}
$$

As a result, for limited motion, the overall latency caused by transmission delay and feature quantization is significantly reduced.

\subsubsection{Database}

In contrast to product recognition applications, we match regular images to spherical panoramas, which depict the complete $360^{\circ}$ scene. Thus, only a fraction of the features that appear 
at a given location (usually around 3000) can be found in the query. Further, as panoramas are usually provided in form of equirectangular projections, objects are considerably distorted as shown in Figures 3.1 and 2.3. Thus, 12 rectilinear, partially overlapping views are generated via gnomonic projection as illustrated in Figure 4.1. Features extracted from these views are fused to represent a single location.

The selection of database features is based on the information gain of each visual word with respect to a certain location [SBS07]. This gain is maximized if the visual word appears in neighboring locations but rarely at any other location. This approach implicitly filters unreliable features like those resulting from moving objects and too generic textures like windows. Experiments on the above described datasets show that comparable retrieval performance can be achieved at about $20 \%$ of the original database size. Thus, a location can be represented with less than 1000 features, which is comparable to the number of features required for a single query image.

For location recognition in dynamic environments that include cars, pedestrians, advertisements, etc., the use of static reference data is suboptimal. A dynamic update of the database can be achieved by integrating new features recorded by users. This requires that the position of the user has been estimated with sufficient reliability, which is determined by the use of probabilistic filtering like particle filters including motion models. Further, a weighting of features based on how often the feature has been matched at a given location or pruning of features that do not get matched is performed. Altogether, the database can be adapted to local changes and hence the influence of dynamic objects reduced.

\subsection{Coarse Prior Knowledge on Query Location}

Ideally, a system for mobile location recognition based on visual data should not be bounded to a confined area but allow for continuous and precise pose estimation at any place.

However, the scalability of state-of-the-art BoF based location recognition systems is limited to a few square kilometers (assuming 12 rectilinear images per panorama and one panorama per 30 square meters on average) and performance decreases notably with increasing database size [ $\left.\mathrm{PCI}^{+} 07\right]$. Further, the size of the inverted file system scales linearly with the number of reference images and would require large amounts of RAM on the server. Covering a city like San Francisco with more than $100 \mathrm{~km}^{2}$ would be an enormous challenge, however, is not necessary in practice as some coarse prior knowledge on the location usually exists. This information could for instance stem from the Cell-ID of the network provider allowing us to determine a position estimate in the range of some hundred meters at most. Integrating this prior knowledge into the location recognition process reduces the required resources with respect to memory as well as query time and increases precision.

In $\left[\mathrm{TXG}^{+} 08\right]$, GPS information is integrated into the localization process by transmitting features found in nearby database images to the mobile device. Query features are matched to these selected features on the client via an approximate nearest neighbor search (ANN) to determine the exact pose of the mobile device. While this strategy efficiently reduces the network latency as prior information is preloaded to the mobile device, it can hardly cope with weak prior knowledge based on, e.g., Cell-IDs. The amount of features to be downloaded to the client would result in significant initialization times as well as high computational complexity on the mobile device. 


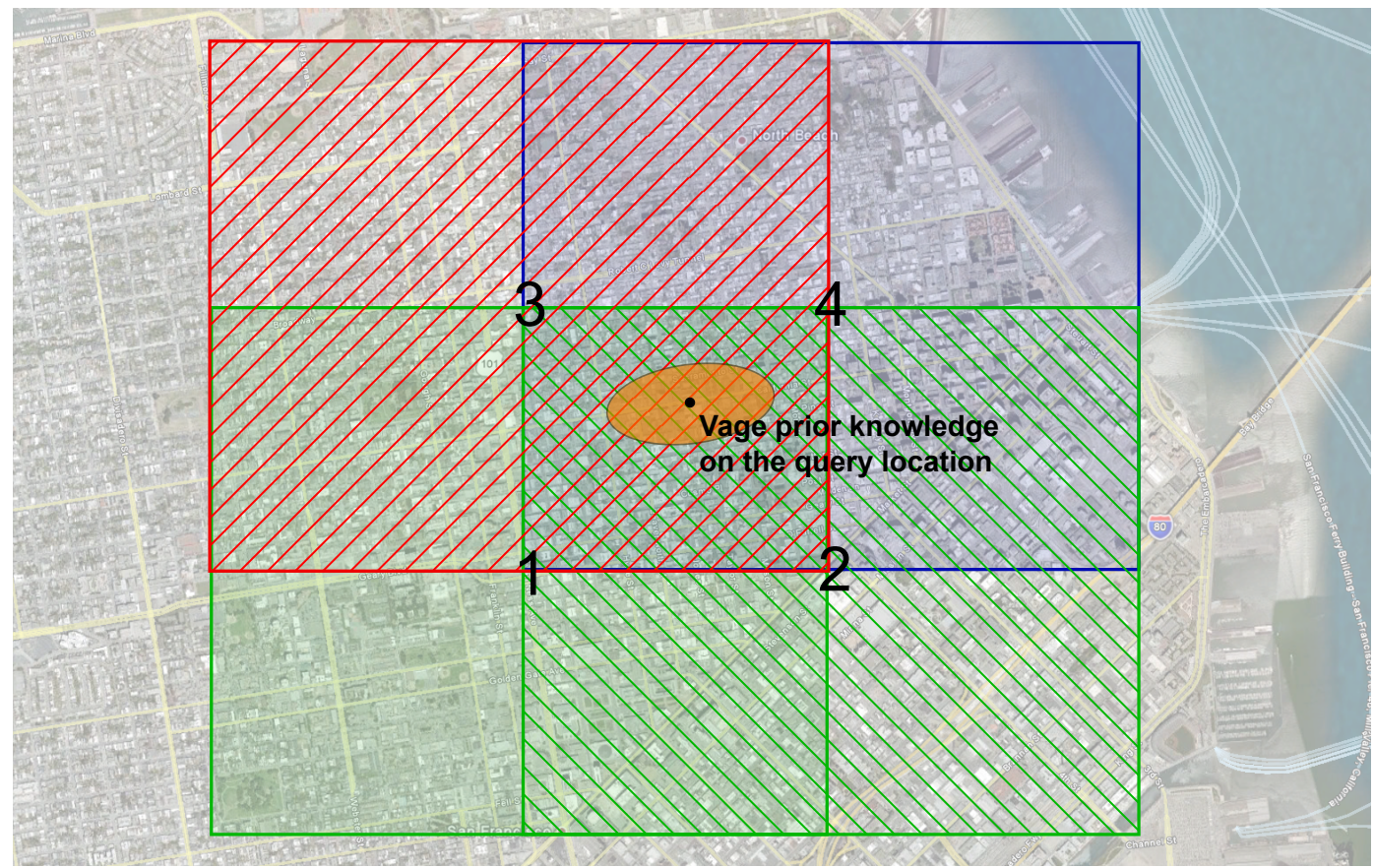

Figure 4.2: Spatial partitioning of the search space in four regions / trees, which overlap by $50 \%$ in horizontal and vertical direction; The double shaded area in the middle is covered by four trees. (Reproduced from [SHC+11], (C) 2011 IEEE)

Chen et al. propose a different approach in $\mathrm{CBK}^{+} 11$, where the GPS information is used to filter the image retrieval results as part of a post processing step. While this approach is efficient and easy to integrate, prior information can be integrated deeper into the matching process to not only improve the precision but also reduce complexity, memory usage, as well as network delay.

Hence, the search area, i.e., a large environment like a city, should be segmented into several overlapping subregions for which individual quantization structures and associated inverted file sets are generated. The size of the subregions can be set to approximately $4 \mathrm{~km}^{2}$ depending on the expected accuracy of the prior location information. In the example in Figure 4.2, the area is covered by four subregions, overlapping with horizontally and vertically adjacent regions by $50 \%$. Thus, every distinct location in the double shaded center is covered by four quantization structures. The pattern is continued accordingly across the whole city or even larger areas, such that for every location in the search area four separate vocabularies exist.

In the localization process only those, usually four, vocabularies are queried that cover locations within the area of the current uncertainty about the position of the user (see Figure 4.2). However, in case of large areas of uncertainty or overlaps with the borders of a subarea, at most nine vocabularies have to be queried, where at least one covers the whole area of uncertainty.

For one database image in the area of uncertainty, this always results in four redundant similarity estimates. Since these similarity estimates are determined by quantization structures built with a significantly differing image set, the quantization results of each are slightly 
different from the others. Nevertheless, the similarity scores are comparable and can be fused for the actual retrieval as described in the following. This is similar to techniques like Locality Sensitive Hashing [DIIM04] or randomized kd-trees [SAH08, where the results of multiple random quantization structures are fused. However, we do not randomize our quantization structure directly, instead we compute tailored quantization structures but for different image sets. Thus, combining the redundant results allows us to significantly increase the precision at the cost of increasing the query time by the number of considered subareas.

In addition to reduced complexity and improved precision, this approach further allows us to provide a seamless handover to adjacent regions. Once a user is approaching the border of the currently covered search space, i.e., the area covered by four quantization structures per subregion, adjacent structures and associated inverted files are preloaded to the RAM of the server. Thus, the position of the user is always close to the center of one of the trees.

The fusion of the retrieval results can be based on the returned similarity scores and the spatial relationship of the retrieved locations. In a first step, the top $K$ results for each query are selected and fused similarity scores are calculated by concatenating the BoF vectors of all queried vocabularies. This is efficiently done by simply summing over all individual similarity scores.

Even though database images are typically more than $12 \mathrm{~m}$ apart, adjacent images are likely to share parts of the scene. Thus, the similarity scores of reference images close to the selected result images indicate whether query features are also matched at different viewing angles. The information implied in local clusters of high similarity is utilized by weighting the scores with the similarity scores of reference images in the direct vicinity.

Finally, at most $K$ locations sorted with respect to the final score are returned. This algorithm effectively increases the precision of the returned results by fusing the information of multiple quantization structures at very low computational complexity.

\subsection{Experimental Evaluation of the Basic Location Recognition Architecture}

To illustrate the performance of a BoF-based mobile location recognition system based on the proposed feature selection and integration of prior knowledge, we query a Google Street View database of San Francisco comprising over $60 \mathrm{k}$ images at a spatial resolution ranging between 12 and $17 \mathrm{~m}$. The realistic query video is recorded along a $0.7 \mathrm{~km}$ long section of the track in Figure 1.1 at a resolution of $800 \times 480$ pixels and an opening angle of $70^{\circ}$. Please note that in some video frames only trees or mostly clutter are visible. Further, occasional motion blur impairs the video quality.

A query is composed of tracked features within a time frame of 5 seconds. We conservatively assume an area of location uncertainty of a radius of $1 \mathrm{~km}$. After $0.5 \mathrm{~km}$ the track crosses the boundary of a subregion and a handover occurs. The top 10 results of the fusion of four subareas with a size of $4 \mathrm{~km}^{2}$ each are considered. In this experiment the retrieval is performed on the server and every query is assumed to have the same coarse prior knowledge about the location of the user. The approach of spatial partitioning, as discussed above, is combined with the AKM algorithm $\left[\mathrm{PCI}^{+} 07\right.$.

Figure 4.3 shows the number of retrieved locations within a given radius around the true 


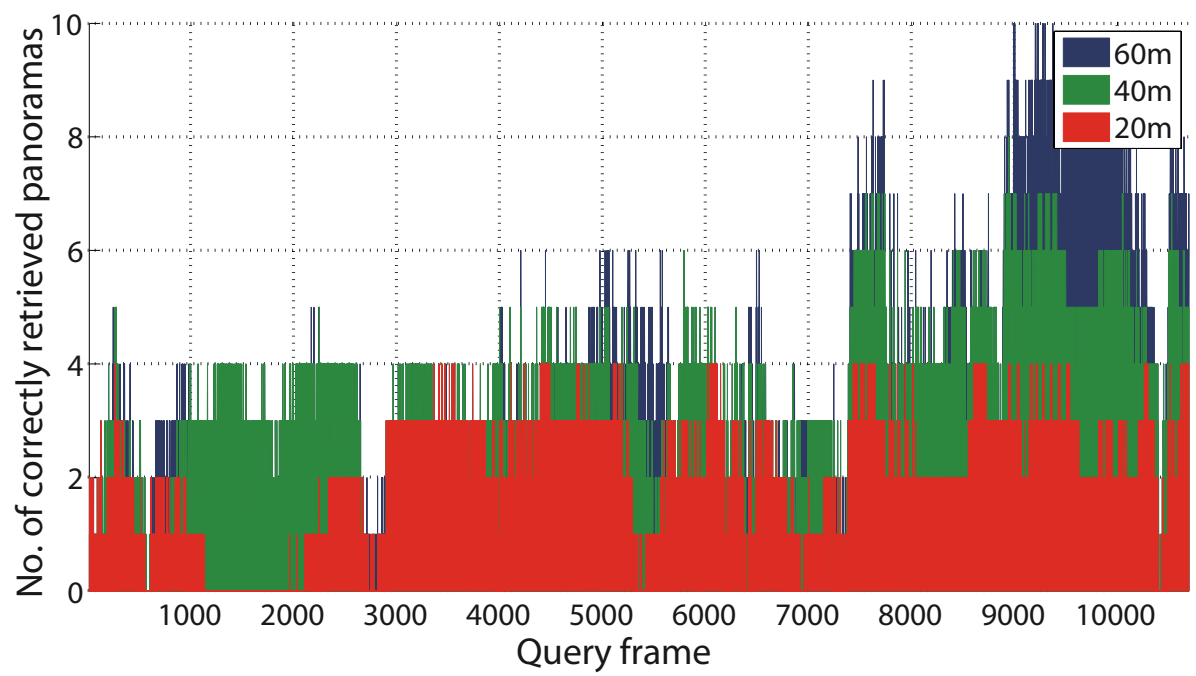

Figure 4.3: Evaluation of retrieval performance for realistic query video recordings. The number of retrieved locations within a given radius around the true query location is plotted against the frame number. (Reproduced from [SHC 11], (C) 2011 IEEE)

query location for the individual video frames. For $79.6 \%$ of the track at least one panorama has been retrieved within $20 \mathrm{~m}$ and for $93.2 \%$ of the track within $40 \mathrm{~m}$. Without the use of virtual feature panoramas and without the removal of green features, as discussed in Section 4.1 . the performance degrades significantly. Only for $34.4 \%$ of the track at least one panorama is retrieved within $20 \mathrm{~m}$. Please note that on average three panoramas are located within a radius of $20 \mathrm{~m}$, except for street junctions. Increasing the radius further results in a marginal increase in correct matches as the visual similarity is local. At frames 7500 to 10500 the video recordings show a square, such that also distant locations are visually similar. Unsuccessful localizations, which occur if the query feature set is degenerated, can be overcome with the aid of probabilistic filtering and visual odometry as described in Section 1.3. Subsequently an image registration can be performed to determine the pose of the mobile device with respect to neighboring panoramas.

With the above described extensions of the basic system architecture in Figure 2.11 the localization performance with respect to precision and recall can be significantly improved. Further, by transferring only selected tracked features from the client to the server, the overall network latency can be reduced to 2-3 seconds depending on the motion of the mobile device. This system forms the basis for the following chapters. 


\section{Elimination of Network Delay and Integration of Prior Knowledge on the Location}

प

Ideally, a user is notified about an LBS in the very moment the camera records the corresponding store, exhibit, trademark, etc. Due to the rapidly changing field of view and dynamically changing user attention, very low system latency is essential for LBS to be perceived as useful. As described above, the latency is mainly determined by the time required for the feature extraction, the quantization into visual words, and the delay caused by the transmission of the data from the mobile device to the server. Several approaches have been proposed to minimize the amount of data to be transmitted to the server. Chandrasekhar et al. $\mathrm{CTC}^{+}$09a] introduced a Compressed Histogram of Gradients (CHoG) descriptor, which exploits the non-uniform distribution of gradients to describe an image patch using only 60 bits. Hence, an image can be represented by $3-4 \mathrm{kB}$ requiring approximately 3 seconds to be uploaded using a $3 \mathrm{G}$ network. Clearly, this is not sufficient for most location based services.

With the approach described in Chapter 3 , the amount of data to be transmitted is reduced by a factor of 5 with respect to $\mathrm{CHoG}$ descriptors $\left[\mathrm{CTC}^{+} 09 \mathrm{~b}\right]$ by quantizing features directly on the mobile device into visual words. As illustrated in Figure 5.1, instead of sending compressed features, visual word indices are transmitted. This, however, requires downloading the quantization structure to the mobile device, which results in a time consuming initialization or to use a generalized quantizer which would lead to inferior retrieval performance. While this approach would be sufficient using a Wi-Fi network, the proposed visual localization system should not require additional infrastructure.

With the described system architecture in Figure 5.1 the response time always suffers from

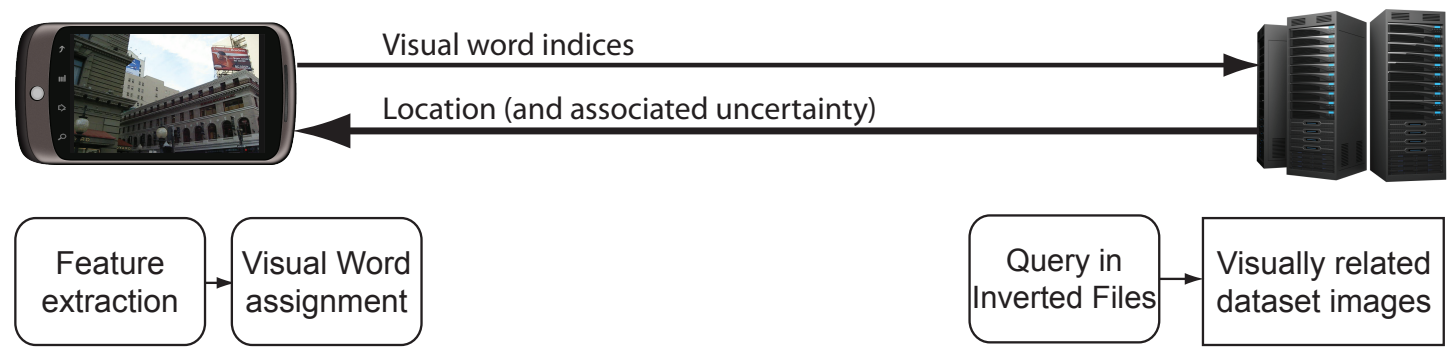

Figure 5.1: Overview of the visual location recognition system architecture of Chapter 3 . (Reproduced from [SHC+11], (C) 2011 IEEE)

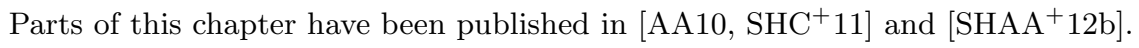




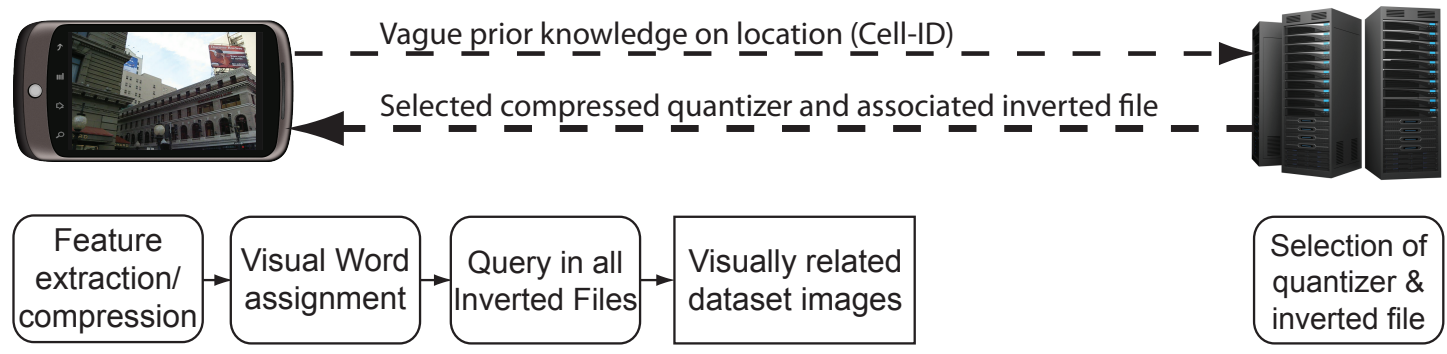

Figure 5.2: Overview of a visual location recognition system where the retrieval task is shifted from the server to the client by transferring the quantization structure and compressed inverted file system of the most suitable subregion. Dashed lines indicate the data transmission during initialization. Once the transfer of the quantization structure and the inverted file is finished, no further network delay is introduced. (Reproduced from [SHC 11], (C) 2011 IEEE)

the round trip time, which ranges between 0.3 and 0.9 seconds on $3 \mathrm{G}$ networks, and timeouts in addition to the time required to actually transfer the data $\left[\mathrm{GCC}^{+} 11\right]$. The delay caused by the communication between the client and the server using a typical $3 \mathrm{G}$ network has been studied in $\left[\mathrm{GCC}^{+} 11\right]$. The transmission of $10 \mathrm{kB}$ using the uplink of an indoor $3 \mathrm{G}$ connection takes about 5 to 8 seconds on average depending on the signal quality. This does not include timeouts, which happen in about $6 \%$ of the connections. In case of dead zones, the localization will instantaneously break down. Further, the architecture in Figure 5.1 almost exclusively uses the slower uplink channel for uploading query features, whereas the downlink is usually more than 5 times faster.

Recently, Ji et al. [JDC $\left.{ }^{+} 11\right]$ proposed to integrate weak prior knowledge on the location to adapt the allowed set of visual word indices to be sent to the server. Based on a generalized visual vocabulary the subset is selected based on ground truth data of previously selected distinctive landmarks. To distinguish among the approximately 60 landmarks per city, less than 32 bits are needed in $\left[\mathrm{JDC}^{+} 11\right.$ ] to represent an image. This approach, however, can be hardly adopted to continuous location recognition at arbitrary query locations as representative exemplary query images can not be generated. Further, even with very limited amount of data to be transmitted to the server, the roundtrip delay of about 0.3 to $0.9 \mathrm{~s}$ can hardly be avoided in this client-server architecture.

In the subsequent sections novel approaches to cope with the two central challenges of mobile visual location recognition are introduced, which are the computationally complex retrieval task and the communication delay.

\subsection{Big Client Architecture}

A possible approach to solve the above described problems is to transfer the quantizer as well as the inverted file to the client and perform the location retrieval exclusively on the mobile device as shown in Figure 5.2. With the approach described in Section 4.2 to integrate coarse prior knowledge, we limit the size of the inverted file system to about 5000 panoramas and 
send a tailored quantization structure. This could be the MHVT quantizer described in the previous chapter or, for example, the approximate k-means algorithm (AKM) [ $\mathrm{PCI}^{+} 08$ ], which uses a flat vocabulary where descriptors are assigned to k-means centroids via randomized kd-trees, as described in Section 2.2.3. For the latter, the centroids of the vocabulary are represented by $\mathrm{CHoG}$ descriptors requiring 60 bits each, to reduce the download time. With the size of a kd-tree being less than $100 \mathrm{~KB}$ for 1 Million centroids, the overall memory requirements range between 3.75 to $7.5 \mathrm{MB}$ depending on the size of the vocabulary.

Additionally, the inverted file needs to be transferred, which can be efficiently compressed following the $\mathrm{BoF}$ compression approach in $\left[\mathrm{CTC}^{+} 09 \mathrm{~b}\right]$. This allows us to reduce the size of the inverted file to about 12.5 MB. Further, spatially neighboring panoramas overlap with respect to the associated visual words by about $18 \%$ depending on their distance and the size of the vocabulary. This is exploited by transferring differentially encoded binary BoF vectors, which include only the difference to the visually most similar panorama. This allows for an additional reduction of the size of the inverted file by more than $10 \%$.

Using the AKM with $4 \mathrm{kd}$-trees and 40 checks on a vocabulary of $500 \mathrm{k}$ words, the assignment of 1000 descriptors to visual words requires about $180 \mathrm{~ms}$ on a Nexus S phone. This query time is reduced to about $50 \mathrm{~ms}$ by quantizing only the newly appearing descriptors depending on the amount of motion as described in Section 4.1.1. An even faster quantization $(12 \mathrm{~ms}$ per 1000 descriptors) is achieved using the MHVT approach introduced in Chapter 3.

Although this approach eliminates the network delay by performing the location retrieval on the mobile device, the amount of data to be transmitted via the downlink would result in a time consuming initialization of the system. During this phase, localization would need to be performed according to Figure 1.3 , where compressed features are sent to the server and the position can be updated approximately every 3 seconds.

\subsection{Partial Visual Vocabularies}

With compressed features being uploaded every 3 seconds, a periodic update of the client position is available at the server. Clearly, the client does not require the full quantization structure and inverted files to allow for a real-time orientation and position estimation until the next update from the server arrives. Rather a dynamically adapted quantizer that integrates the prior knowledge on the position should be employed. In the following the partial vocabulary approach is described, which exploits the typically 5 times faster downlink to preload selected reference data to the mobile device. This allows us to integrate prior knowledge, reduce computational complexity and to perform the localization within a limited area without waiting for responses from the server.

As shown in Figure 5.3, the features of every $k$ th frame are periodically transmitted to the server. To minimize the time required to upload the data, compressed $\mathrm{CHoG}\left[\mathrm{CTC}^{+} 09 \mathrm{a}\right]$ descriptors are used. Multiple vocabularies, each covering an area of about $5 \mathrm{~km}^{2}$ comprising approximately 6000 panoramas, are available at the server. Based on the weak prior knowledge a tailored vocabulary is selected and used to quantize the uploaded features into

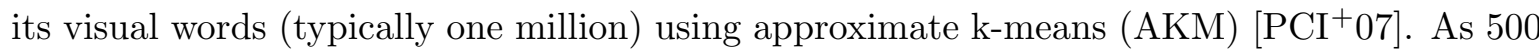
CHoG descriptors can be uploaded in about 3 seconds, the server can determine the visually most similar panorama and thus the location of the mobile device typically every 90th frame assuming $30 \mathrm{fps}$. With these periodic location estimates, we can limit the reference data re- 


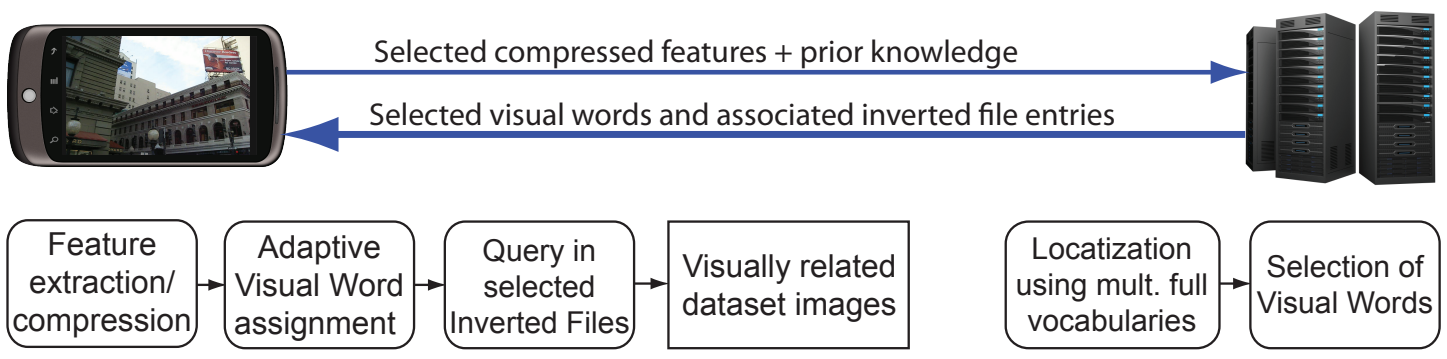

Figure 5.3: Overview of a visual location recognition system where relevant parts of the vocabulary are periodically pushed to the client. The selection of the transmitted visual words is based on the location retrieval performed at the server using the query features uploaded from the client. The pose estimation is performed on the mobile device utilizing these preloaded adaptive partial vocabularies. Blue lines indicate that the network delay does not influence the time required for the location recognition. (Reproduced from [SHC 11], (C) 2011 IEEE)

quired at the client to localize itself until the next server-based estimate to a fraction of the full vocabulary and inverted file system.

If we consider $F=\left\{f_{1}, f_{2}, \ldots, f_{N}\right\}$ to be the set of features of one query frame and $V=$ $\left\{v_{1}, v_{2}, \ldots, v_{L}\right\}$ to be the set of visual words (i.e., the full vocabulary), the quantization function $q_{V}\left(f_{i}\right)=v_{j}$ assigns each feature $f_{i}$ to the closest visual word $v_{j}$ in the full vocabulary $V$. This is typically done by performing a nearest neighbor search among all visual words. The subset of visual words, which represents a particular video frame, is defined as:

$$
Q(F \mid V)=\left\{v=q_{V}(f) \mid f \in F\right\}=V_{F} \subseteq V .
$$

The result of exact nearest neighbor quantization remains unchanged if only the subset of visual words representing the frame itself $V_{F}=Q(F \mid V)$ is used instead of the full vocabulary:

$$
Q\left(F \mid V_{F}\right)=Q(F \mid V)
$$

Figure 5.4 sketches the quantization of descriptors into the subset $V_{F}$ using a Voronoi diagram. In Figure 5.4a the full vocabulary with the centroids drawn in black dots is used for the quantization resulting in the Voronoi cells indicated by the blue vertices. The distance to the closest centroids is identical for points lying on vertices of Voronoi cell. Selecting a subset of the full vocabulary marked as green dots in Figure 5.4b results in enlarged Voronoi cells and thus a coarser quantization in Figure 5.4c. However, due to the quantization via nearest neighbor assignment, the enlarged cells are guaranteed to completely contain all descriptors assigned to the respective centroid using the full vocabulary (illustrated by the yellow areas in Figure 5.4d).

Hence, only the subset $V_{F}$ of the full vocabulary needs to be available at the client to obtain the same results. However, this equation only holds for a specific set of features $F$. A partial vocabulary $V_{F}$ would need to be sent to the client for each frame. Ideally, we would 


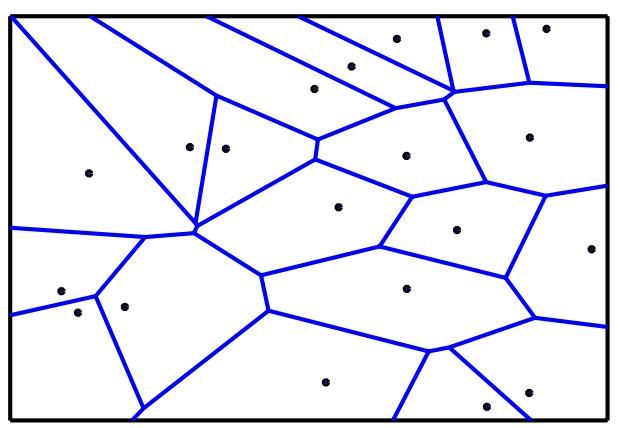

(a)

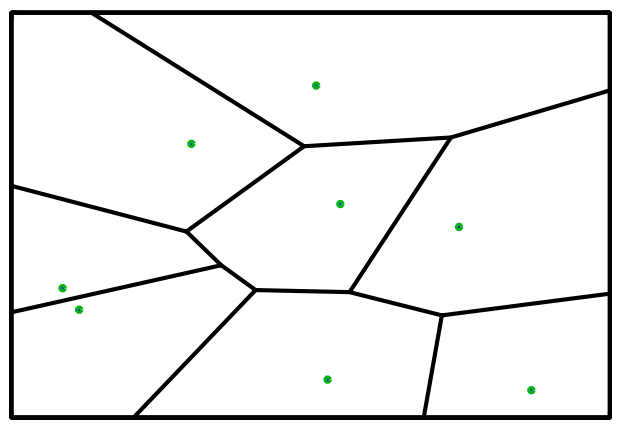

(c)

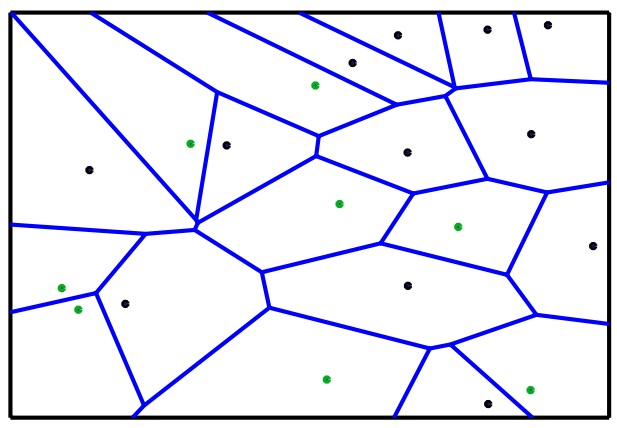

(b)

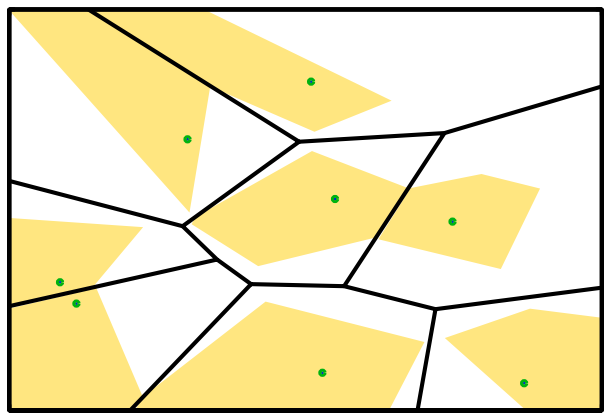

(d)

Figure 5.4: Quantization using partial vocabularies. Limiting the quantization to a subset (green dots) of the full vocabulary results in enlarged Voronoi cells that fully contain the cells of the full vocabulary (yellow areas).

like to identify a partial vocabulary that includes the correct visual words to process multiple consecutive frames without the need to know their features a priori. Since $V_{F}$ can be extended by other subsets of the full vocabulary $(S \subseteq V)$ without changing the quantization result, as shown in Equation 5.3, we can use partial vocabularies at the client that have a sufficiently high probability of including $V_{F}$ for the next upcoming frames.

$$
Q\left(F \mid V_{F} \cup S\right)=Q\left(F \mid V_{F}\right)=Q(F \mid V)
$$

To limit the amount of data to be transferred to the client, we seek for the smallest partial vocabulary that includes the unknown $V_{F}$ for the next video frame(s) with high probability.

\subsubsection{Composing the Partial Visual Vocabularies}

While the field of view of the mobile device may change rapidly due to the varying user attention, the location typically changes only at a walking pace of about $1.2 \mathrm{~m} / \mathrm{s}$. Thus, visual words representing successive frames are found with a high probability in the same reference image, i.e., a panorama, at the corresponding location, which is periodically retrieved at the server every $k$ th frame. As the distance between neighboring panoramas ranges between 12 to $17 \mathrm{~m}$, the visual words of two panoramas are sufficient for the client to localize itself for about $10 \mathrm{~s}$. To increase the time the client can navigate based on the partial vocabulary, visual 
words of panoramas that are adjacent to the top $K$ location estimates are added. Thus, the set $S$ in Equation 5.3 is composed of the visual words from the top ranked $K$ panoramas retrieved by the server and their neighboring panoramas within a radius $R$.

While this approach does not ensure to include the complete sets $V_{F}$ of the next video frames in the partial vocabulary, this interestingly improves performance as it effectively integrates prior knowledge on the location into the quantization process at the client. Features can only be quantized into visual words located in the area of the current location uncertainty. Further, due to the small size of partial vocabularies, the quantization of features can be performed at high rates on the mobile device, achieving $10 \mathrm{fps}$ including feature extraction on a state-of-the-art phone.

As described above, features that vote for one of the preselected locations are matched to the same visual words as if using the full vocabulary. Only those features that refer to other locations with the full vocabulary can happen to be matched to a different visual word when considering a partial vocabulary. Applying a threshold on the maximally accepted distance between the feature and the visual word does not lead to an improvement. The enlarged Vornoi cells of partial vocabularies (see Figure 5.4 rather increase robustness with respect to descriptor variances. Assuming the prior knowledge on the location is correct, partial vocabularies ensure that the query descriptors are assigned to one of the visual words occurring in the current location and are less likely to be incorrectly quantized to one of the neighboring centroids in descriptor space.

The number of visual words to be downloaded depends on the currently available data rate and the time until the next localization update at the server (termed step size). As the visual words and their associated inverted files are progressively downloaded and added to the quantization structure, the visual words included in the closest panoramas are transmitted first. The radius $R$ is set according to the expected distance travelled until the next server update. Less time has to be spent on downloading the visual words from neighboring panoramas as they typically share about $20 \%$ of their visual words.

To compare the performance of this system, we use the very same Google Street View dataset as in Figure 4.3. The realistic video recording along the $0.7 \mathrm{~km}$ long track is processed at 10 fps. Every 3 seconds a new set of tracked query features is uploaded to the server and used to query the full vocabularies to retrieve the visually most similar locations. Based on these recognized location candidates, the partial vocabulary is updated and at most 5000 selected visual words and their associated inverted files entries are downloaded to the client within 3 seconds. The pose estimation is continuously performed on the mobile device at $10 \mathrm{fps}$ utilizing the adaptive partial vocabulary as illustrated in Figure 5.3 .

As in Figure 4.3, we plot the number of retrieved locations within a given radius around the true query location for the individual video frames in Figure 5.5. The use of adaptive partial vocabularies on the mobile device allows us to retrieve at least one panorama within $20 \mathrm{~m}$ for $82.3 \%$ of the track and within $40 \mathrm{~m}$ for $91.0 \%$ of the track when considering the top 10 retrieved locations. As shown in Figure 5.5 the number of retrieved locations within the given radius is significantly increased by adaptive partial vocabularies. No temporal smoothing approaches like particle filters or geometric verification techniques are applied in this evaluation. The composition of the partial vocabulary is exclusively based on the most recent set of features uploaded to the server.

Using a partial vocabulary composed of the top ranked $K$ panoramas and their neighbors 


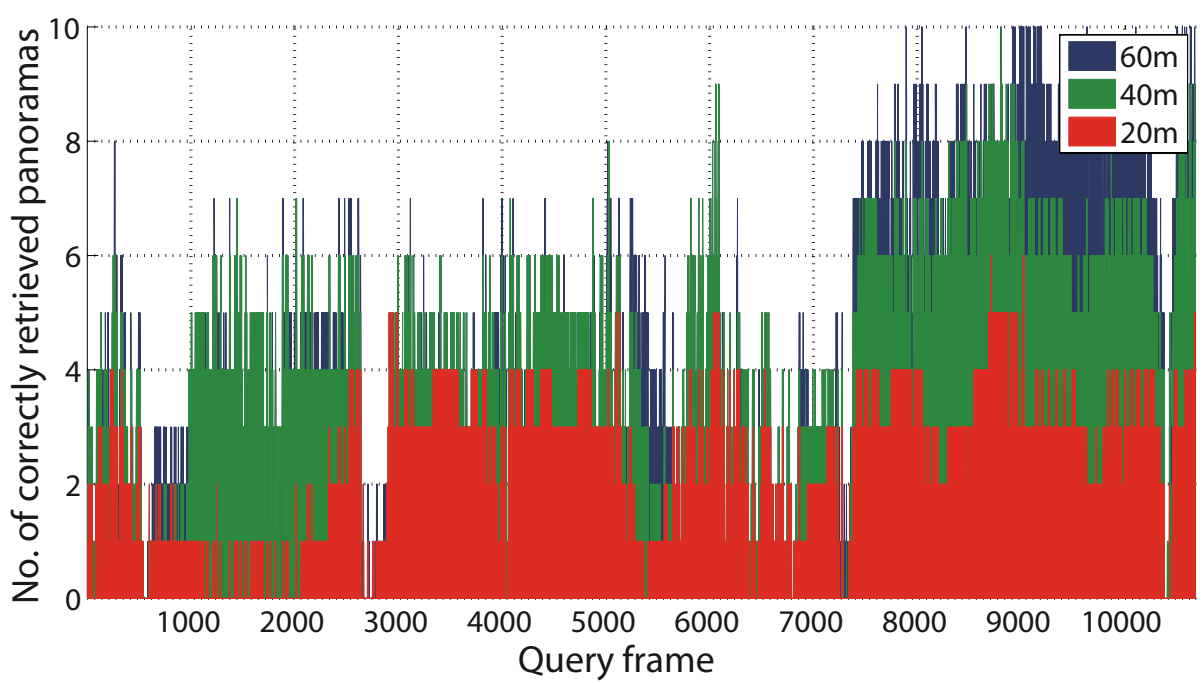

Figure 5.5: Evaluation of the location recognition performance of adaptive partial vocabularies using the same dataset and metrics as in Figure 4.3. A significant increase in the number of correctly retrieved panoramas per query location can be observed. (Reproduced from [SHC 11], (C) 2011 IEEE)

within a radius $R$ yields very good results but exploits only the information obtained from a single set of features uploaded to the server. The area of location uncertainty can be better estimated using a particle filter which fuses the information obtained from consecutive query sets.

\subsubsection{Particle Filter Based Partial Vocabularies}

By tracking multiple particles, i.e., hypotheses, on the pose and velocity of the mobile device at the server, we can fuse consecutive retrieval results by exploiting a constant velocity model or even integrating visual odometry as described in Section 1.3. As the client can be assumed to have a zero centered Gaussian distributed acceleration, a given maximum velocity and rate of turn, the motion model allows us to predict possible locations until the next retrieval update becomes available. Based on the state vectors of the last set of particles, new hypotheses are generated, which are samples of the probability density function (pdf) of the estimated client location. In addition, single particles are added at the location of the last retrieved panoramas. This allows us to reduce the number of particles and to account for distant but consistent retrieval results.

Every time a new set of features arrives at the server, the particles are weighted according to the probability that the retrieval results are obtained at their location. Using a ground truth dataset a pdf that specifies the probability of retrieving a given panorama at a given distance has been learned. This pdf is specific to a given retrieval pipeline. As subsequent updates occur typically every 5 to 25 seconds, the temporal correlation of the noise in the retrieval results is small. Thus, false retrieval results are ruled out by the filter as their associated locations do not comply with the motion model. Based on this low complexity particle filter, 


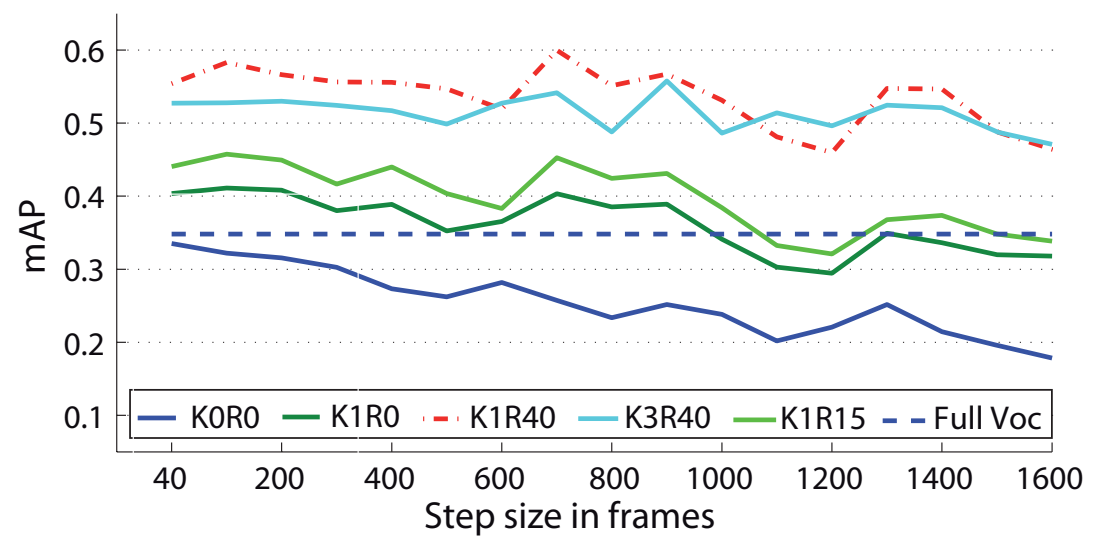

Figure 5.6: $m A P$ scores for multiple partial vocabulary configurations over the update rate (step size). (Reproduced from [SHAA+12b], (C) 2012 IEEE)

we can avoid time consuming geometric verification of the retrieval results at the server.

Based on the resulting pdf over the estimated client location the partial vocabulary of visual words that best covers this area is composed. As additionally the direction of motion as well as the velocity is estimated, significantly fewer visual words have to be transmitted to achieve the same or better retrieval results at the client.

Further, by utilizing database statistics those visual words are identified that provide the maximum amount of information to distinguish individual locations [SBS07] and, at the same time, are most frequently found at a specific location. This allows us to reduce the number of visual words representing a panorama by at least a third at hardly any loss in retrieval performance. This way, less than 1000 visual words are sufficient to represent a full panorama.

Visual words are represented by compressed CHoG descriptors (60 bit) and inverted file entries are encoded differentially $\left[\mathrm{CTC}^{+} 09 \mathrm{~b}\right]$ to further reduce the amount of data to be downloaded. On average a total of 140 bit per new visual word and associated inverted file entries (typically 6 in a database of 6 thousand images $\left(5 \mathrm{~km}^{2}\right)$ and 1 million visual words) has to be downloaded. However, as more and more visual words are already available at the client, only their index (20 bits) has to be transmitted to form the partial vocabulary at the client. The data transmission will be discussed in more detail in the following section.

\subsection{Experimental Evaluation}

In the following we evaluate the performance of our approach in detail using the same dataset as in Figures 4.3 and 5.5. In this evaluation, however, the query recordings are processed at $30 \mathrm{fps}$ to better display the performance over time. The camera motion during recording ranges between 0.5 to $1.9 \mathrm{~m} / \mathrm{s}$. Recordings are impaired by motion blur and a severe amount of clutter like cars and foliage. Prior knowledge derived from Cell-IDs allows us to select one of the overlapping $5 \mathrm{~km}^{2}$ subareas at the server which includes 6 thousand Google Street View panoramas with an inter panorama distance ranging from 12 to $17 \mathrm{~m}$.

Every $k$ frames (k is called step-size) features are sent from the mobile device to the server. We extract MSER features [MCUP04] from the least blurry frames within the last $10 \%$ of the 


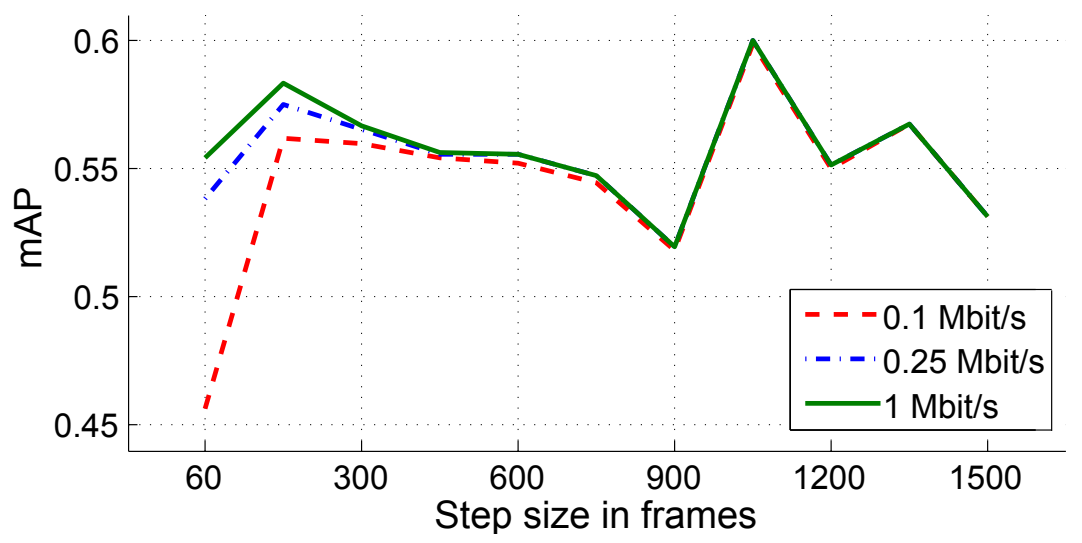

Figure 5.7: $m A P$ score of the K1R40 partial vocabulary configuration at three different data rates over the update rate (step size). (Reproduced from [SHAA+12b], (C) 2012 IEEE)

step size. Based on the retrieval results at the server, which employs an $\mathrm{AKM}\left[\mathrm{PCI}^{+} 07\right]$ with 1 million visual words, the partial vocabularies are formed as described in Section 5.2.1. The client performs feature extraction and retrieval in the partial vocabulary at $10 \mathrm{fps}$ to continuously determine its pose without interference by the server. Query features are matched to the visual words using a forest of four so-called extremely randomized kd-trees GEW06 as a quantization structure that have to be only rarely updated and downloaded from the server. This can be done by replacing one of them at a time, each requiring about $10 \mathrm{kB}$. Visual words are continuously mounted to and unmounted from the leafs of the four kd-trees to adapt the partial vocabulary.

The retrieval and thus localization performance at the client is measured using the widely used mean average precision (mAP) score, where all relevant panoramas have to be ranked first in the list of retrieval results to obtain a score of 1 . It can be interpreted as the area under the precision recall curve introduced in Section 2.2.3. Combining the performance measures precision and recall into a single metric eases the evaluation of parameters with respect to their influence on the performance. In the following experiments we define all panoramas within a radius of $25 \mathrm{~m}$ around the query location to be relevant. Ground truth has been recorded using a state-of-the-art GPS receiver with manual post processing to correct for errors caused by multipath effects.

In Figure 5.6 we evaluate the performance of different configurations of composing the partial vocabulary. As described in Section 5.2.1 $K$ refers to the number of top ranked panoramas and radius $R$ defines the maximum distance of panoramas whose visual words should be downloaded to the client. As neighboring panoramas overlap significantly with respect to the visual words, they are transmitted before sending the visual words of the next best ranked panorama. In this experiment the data rate is not limited and thus all selected visual words are transmitted. The step size defines the number of frames until the server receives the next set of features from the client and updates the list of visual words to be downloaded. With increasing step size, the knowledge about the location of the client, which has to localize itself during this time period based on the partial vocabulary, decreases. Thus, when sending only the visual words that represent the feature set sent to the server (K0R0), the performance decreases 


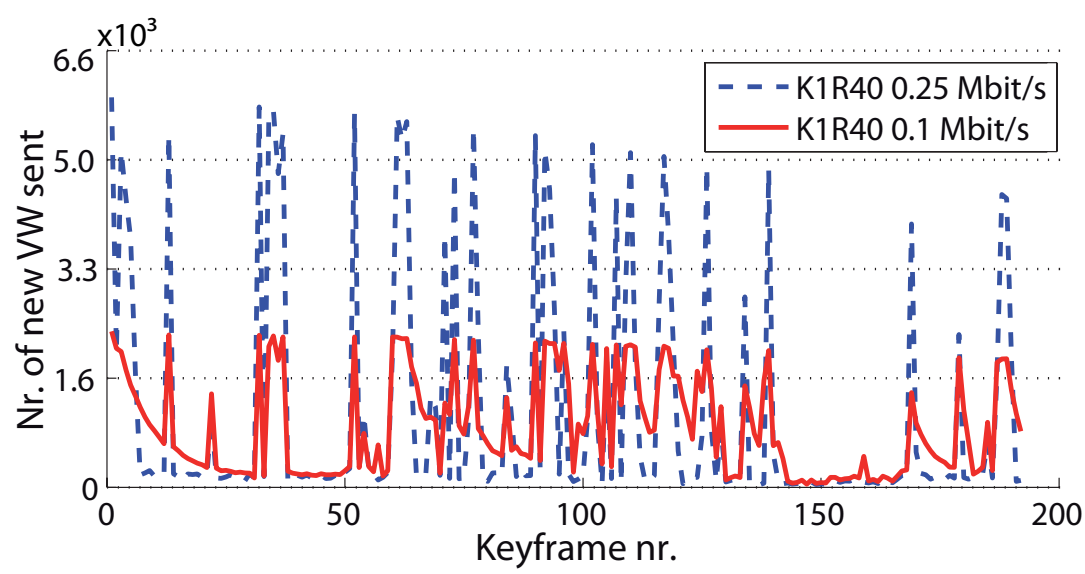

Figure 5.8: Number of new visual words sent to the client per update (key frame) at two different data rates and a step size of 100 frames. (Reproduced from [SHAA+12b], (c) 2012 IEEE)

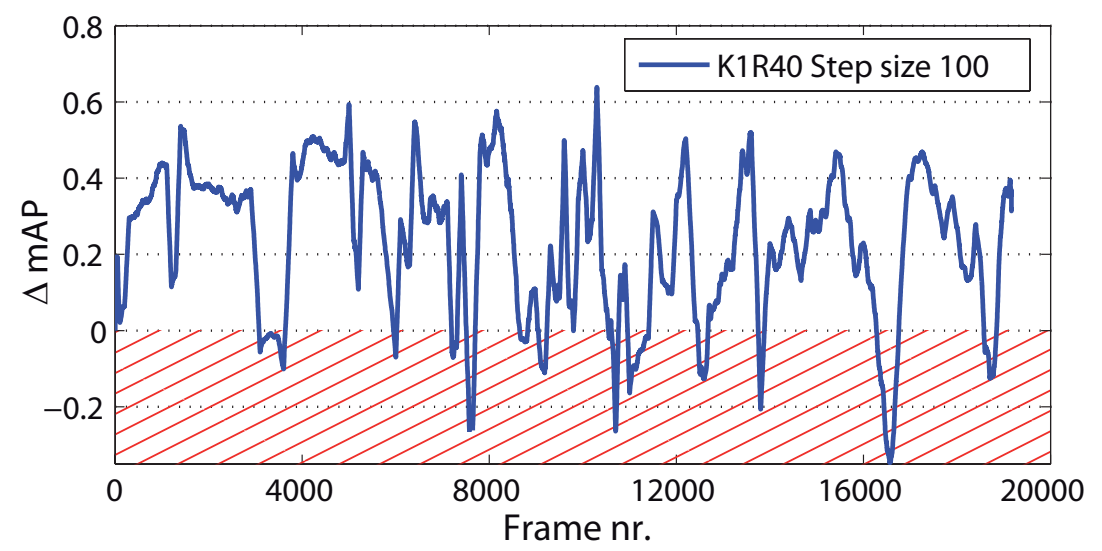

Figure 5.9: Change in $\mathrm{mAP}$ for the K1R40 configuration at a data rate of 0.25 Mbit/s w.r.t. using the full vocabulary at individual frames. (Reproduced from [SHAA+12b], (c) 2012 IEEE)

already at the next frame. The best performance at almost all step sizes is achieved when sending the visual words of the best ranked retrieval result and the neighboring panoramas within a radius of $40 \mathrm{~m}$ (K1R40). Even at a step size of 1000 frames, where the client has to localize itself for 33 seconds based on one update from the server, we achieve a mAP of 0.53 which clearly outperforms the case when using a full vocabulary achieving an mAP of 0.34. A further increase of the partial vocabulary (K3R40) reduces the integration of prior knowledge and would ultimately lead to the performance of the full vocabulary. When including panoramas within a radius of $40 \mathrm{~m}$ the client can localize itself for about $55 \mathrm{~m}$ or about 1400 frames. After this point the performance drops for all configurations.

Assuming multiple clients sharing the bandwidth of a $3 \mathrm{G}$ network, we restrict the downlink data rate to 0.1, 0.25 and $1 \mathrm{Mbit} / \mathrm{s}$ in Figure 5.7. At larger step sizes the client has sufficient 


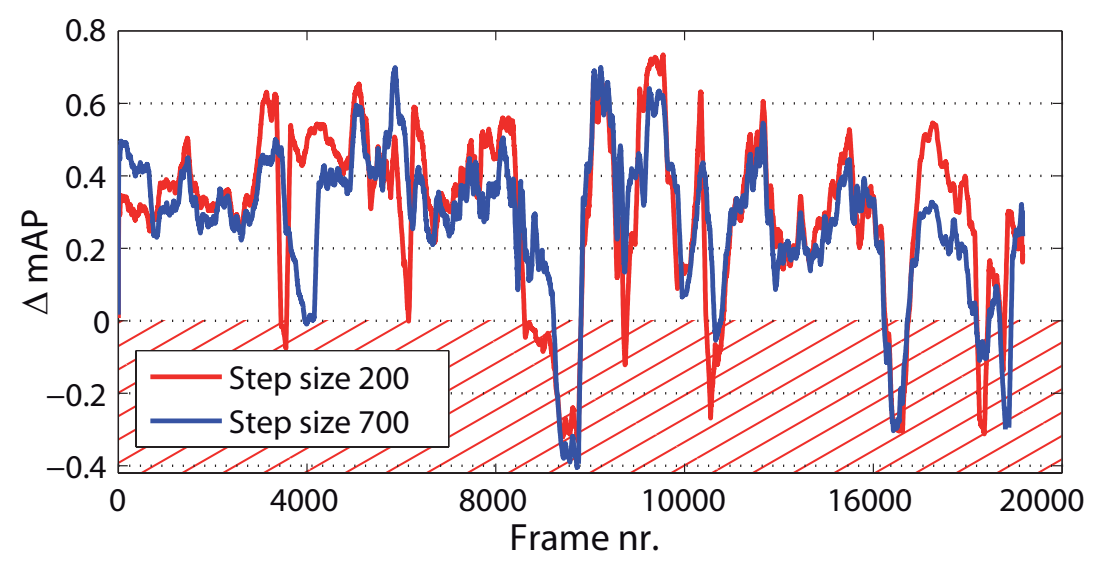

Figure 5.10: Change in $\mathrm{mAP}$ using particle filter based partial vocabularies at $0.25 \mathrm{Mbit} / \mathrm{s}$ with respect to using the full vocabulary at individual frames. (Reproduced from [SHAA+12b], (c) 2012 IEEE)

time to continuously download the visual words and to integrate them into the retrieval system. However, for very short step sizes the rapid change of partial vocabularies can not be transmitted at the limited data rate. On average 9 panoramas can be found within a radius of $40 \mathrm{~m}$ resulting in about 6000 distinctive visual words. As shown in Figure 5.8, this number of visual words only has to be transmitted if the location estimate at the server differs significantly from the last update. Thus, usually only the ids of the already sent visual words have to be transmitted. Further, with increasing time the number of visual words and associated inverted file entries increase which can be observed in the decay of the peaks in Figure 5.8. Visual words that cannot be sent within the time of one update step might be required in the subsequent update and need to be transmitted as can be well observed when limiting the data rate to $0.1 \mathrm{Mbit} / \mathrm{s}$.

To evaluate the performance of the approach over time we compute the mAP scores using a sliding window of 100 frames and compare them to the corresponding mAP scores of the full vocabulary in Figure 5.9. For most frames a significant performance improvement can be observed. A reduced mAP score can occur when the retrieval results of the set of features uploaded to the server were poor and thus affect the partial vocabulary at the client.

When using the particle filter based composition of the partial vocabulary, as described in Section 5.2.2, a more stable and reliable estimate of the location can be computed at the server. This results in an additionally improved (overall mAP of 0.65 ) and less varying performance as shown in Figure 5.10. Further, the number of visual words to be downloaded is significantly reduced to about 3000 as the area of location uncertainty is determined more precisely. This approach also facilitates very infrequent updates by the server with a step size of more than 700 frames, which results in an overall mAP of 0.62 .

\subsection{Summary}

The system proposed enables a close to real-time pose estimation on the mobile device by eliminating the network delay, which would otherwise result in response times of 3-4 seconds 
when using the system architecture in Figure $5.1\left[\mathrm{GCC}^{+} 11\right]$. This allows LBS to react to the constantly changing field of view and user attention. While still obtaining a location update by the server every 3-4 seconds, this architecture additionally exploits the faster downlink to periodically transfer the vocabulary required to navigate within a limited area. Partial vocabularies are limited to the area of uncertainty about the location of the mobile device, which can be derived from features that are infrequently uploaded to the server. This not only reduces the amount of data to be downloaded but also significantly increases the retrieval performance as prior knowledge is integrated into the quantization process.

Thus, the idea of partial vocabularies can also be used in other applications where prior knowledge should be integrated. Database statistics are exploited to send only those visual words to the client that provide most information about the location. Using a particle filter to fuse successive retrieval results at the server allows us to determine the area of uncertainty more precisely and thus increase the performance while reducing the amount of data to be downloaded.

Further, as humans usually move with about $1.2 \mathrm{~m} / \mathrm{s}$ but rapidly change their orientation, the set of query features alters significantly over time, whereas the vocabulary required to cover the change in position is almost constant. Experiments show that after a few minutes a significant fraction of the vocabulary has been downloaded and more and more visual words do not need to be transferred anymore. As selected features are uploaded to allow the server to select the right partial vocabulary, these features can be also used to update the database as part of the online map learning process described in Section 1.2 


\section{Visual Phrase Based Content Based Image Retrieval}

In the preceding chapters novel approaches to overcome major challenges of visual location recognition are presented. These include the reduction of the computational complexity especially on the mobile device, the elimination of the network delay, and the integration of prior knowledge on the user location. Further, system aspects like the composition of the database as well as the queries are shown to be critical for the performance. This is particularly important if only few features are available in the environment, in which we want to navigate. Clearly, the most interesting application of the proposed visual localization system is indoor navigation, where hardly any GPS is available. Especially indoor environments, however, typically provide only few distinctive features, which are to a large extent located on direction signs, door signs, posters, etc. Most of them include some kind of writing. Text is a major source of information for humans to orient and to obtain contextual knowledge about their environment. The combination of characters into strings provides virtually unlimited distinctiveness and thus information.

So far, text in natural images has been largely ignored as a source of information in visual localization. This is mostly due to the high requirements with respect to the image resolution and the computational complexity of state-of-the-art optical character recognition systems (OCR) like Tesseract [Smi07]. While in product recognition applications more time can be spent on the recognition task and complete images can be uploaded to a powerful server farm, location recognition should be performed for the most part on the mobile phone to avoid network delays as described in Chapter 5. Hence, only limited computational power can be spent on OCR. Further, while in product recognition close-to-frontal images at high resolution with hardly any occlusions are available, in location recognition tasks only parts of a store sign might be visible.

To make use of text in natural images, we do not need to translate captured writings into letters. We actually even lose information by converting writings into letters as the specific and unique appearance and thus distinctiveness is lost. In Figures 6.1 a and 6.1 b, one and the same text "cafe" has been recorded at two different locations. Only the unique appearance (different font, color, background, etc.) allows us to differentiate them. It is the variety of fonts that makes character recognition a tough problem. However, when considering the appearance, it adds a substantial amount of additional distinctiveness.

By describing individual characters using local feature descriptors like SIFT or SURF [Low04, BETG08, we can avoid the complex task of text recognition and require only the detection of text locations in the image, which not only reduces the computational complexity but also reduces requirements on the image resolution. Detecting a character is less complex and more robust than differentiating for example a "D" from an "O". Writings are now matched by comparing the individual character (feature) descriptors with each other.

Parts of this chapter have been published in [Hil11, $\left.\mathrm{SHH}^{+} 11, \mathrm{CTS}^{+} 11\right]$ and $\left.\mathrm{TCC}^{+} 11\right]$. 


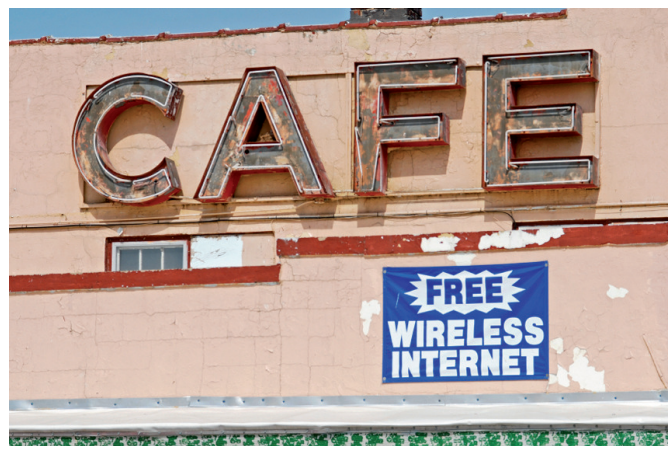

(a)

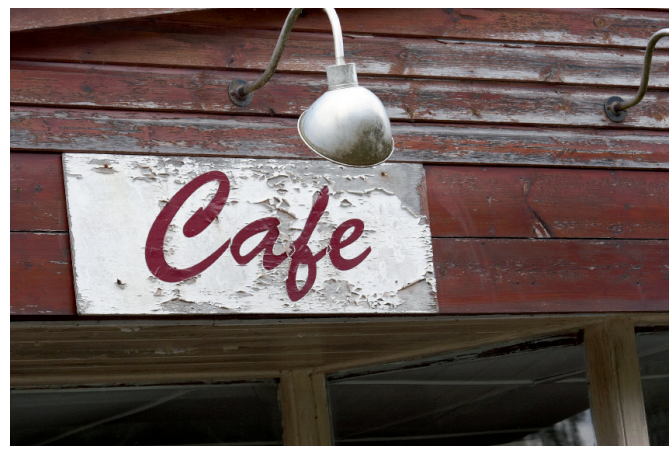

(b)

Figure 6.1: Exploiting not only the information given by the text but also its unique appearance by describing characters as visual words allows us to differentiate the two cafe signs. (Reproduced from [SHH 11], (C) 2011 IEEE)

As writings can be assumed to be on planar surfaces, a strict linear ordering of the individual characters represented by feature descriptors can be derived, which allows us to combine individual character descriptors into visual phrases. Analogous to regular text, this exponentially increases the distinctiveness with the number of characters within the phrase. By using feature descriptors instead of an alphabet of 30 letters, the amount of information is significantly increased.

The remainder of this chapter is structured as follows. The following section introduces a novel MSER based text detector, which is the basis for the Bag-of-Phrases based image retrieval approach. Section 6.2 describes how the proposed approach exploits text related features for CBIR in detail and provides an analysis of the two main parameters of the system. In Section 6.2.1, an overview of related work with respect to text related image and document retrieval is provided. The novel approach is tested and evaluated on multiple datasets in Section 6.3. Finally, we conclude with an outlook to future work and possible extensions for the system in Section 6.4

\subsection{Text Detection in Natural Images}

As described above, the robust detection of text within an image is an essential prerequisite for text-based image search. However, this is a challenging task due to the wide variety of text appearances, such as variations in font and style, geometric and photometric distortions, partial occlusions, and different lighting conditions. Text detection has been considered in many recent studies and numerous methods are reported in the literature LDL05, JKJ04, ZZJ00, YHGZ05, CY04, CY05, Luc05, EOW10, SPT11, LW02. These techniques can be classified into two categories: texture-based and connected component (CC)-based.

Texture-based approaches view text as a special texture that is distinguishable from the background. Typically, features are extracted over a certain region and a classifier trained, using machine learning techniques or by heuristics, is employed to identify the existence of text. In [ZZJ00], Zhong et al. assume that text has certain horizontal and vertical frequencies and extract features to perform text detection in the discrete cosine transform domain. Ye 


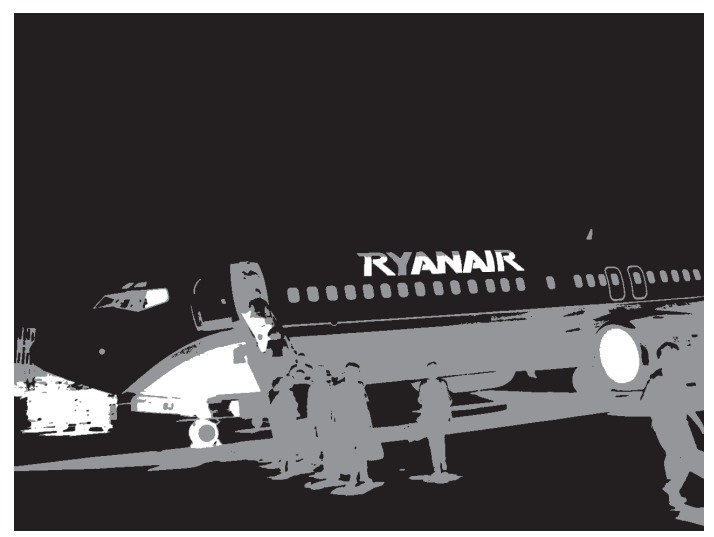

(a) Detected MSER

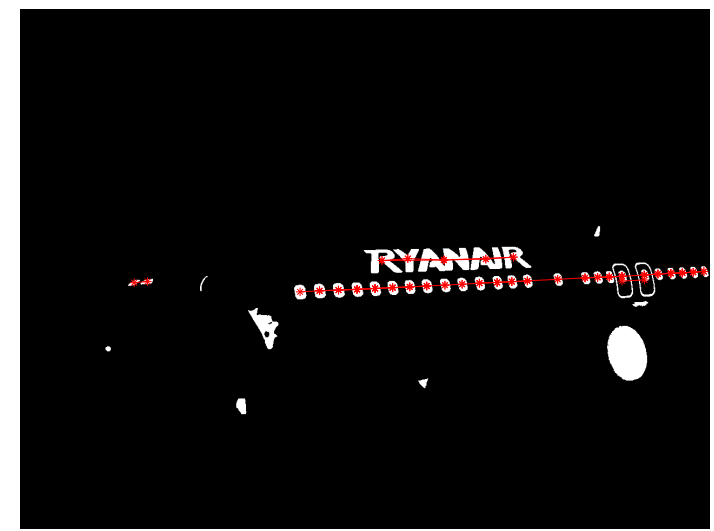

(b) Text candidates

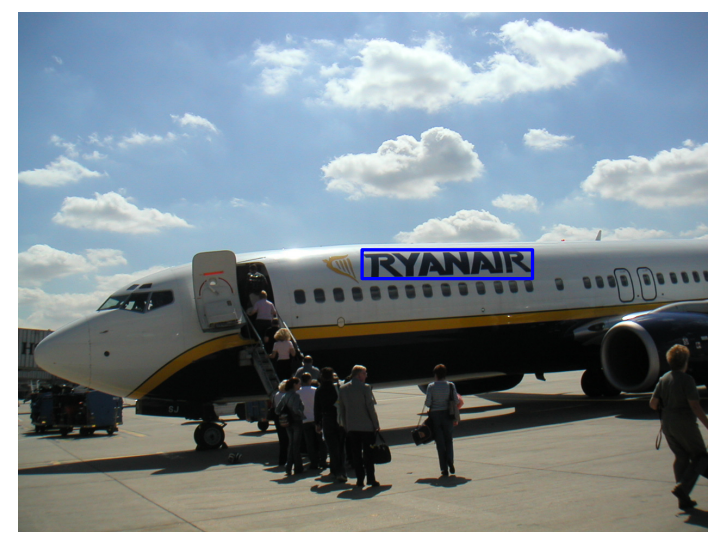

(c) Detected text

Figure 6.2: Extracting text from a natural image. (a): Detected MSER for dark objects on bright background. (b): After geometric and stroke width filtering, text candidates are pairwise grouped to form text lines. The text lines are shown by the red lines. (c): Text line verification rejects false positives and the detected text is highlighted by the blue box. (Reproduced from [CTS 11], (C) 2011 IEEE)

et al. extract features from wavelet coefficients and classify text lines using SVM [YHGZ05]. Chen et al. feed a set of weak classifiers to the Adaboost algorithm to train a strong text classifier [CY04, CY05].

As opposed to texture-based methods, the CC-based approach extracts regions from the image and use geometric constraints to rule out non-text candidates. The top scoring contestant in [Luc05] applies an adaptive binarization method to find CCs. Text lines are then formed by linking the CCs based on their geometric properties. Recently, Epshtein et al. [EOW10] proposed using the CCs in a stroke width transformed image, which is generated by shooting rays from edge pixels along the gradient direction. Shivakumara et al. extract CCs by performing k-means clustering in the Fourier-Laplacian domain, and eliminate false positives by using text straightness and edge density [SPT11].

This section presents a novel CC-based text detection algorithm, which employs Maximally Stable Extremal Regions (MSER) [MCUP02] as basic letter candidates. Despite their favor- 


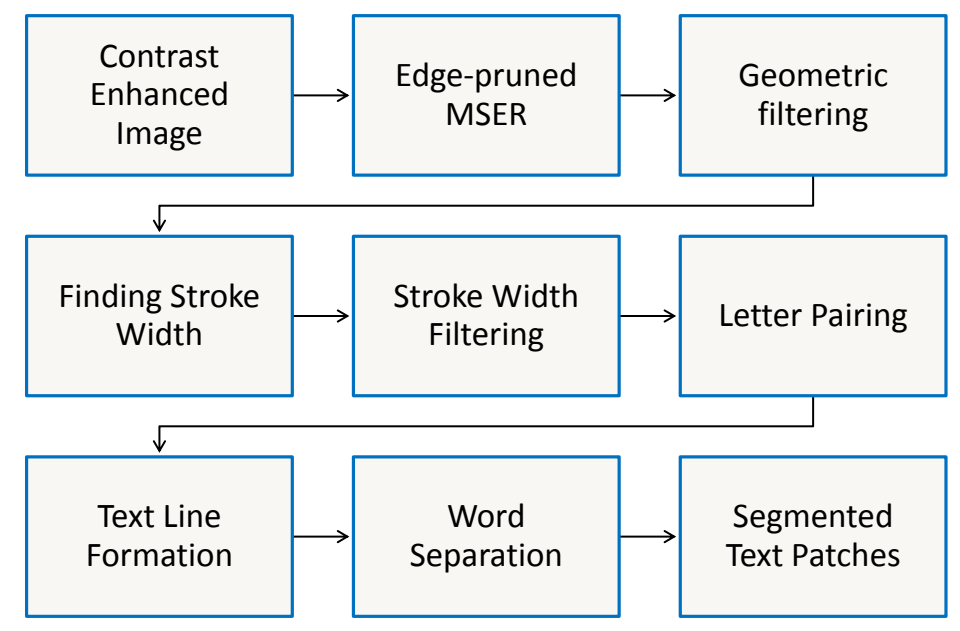

Figure 6.3: System flowchart. (Reproduced from [CTS 11], (C) 2011 IEEE)

able properties, MSER have been reported to be sensitive to image blur [MTS ${ }^{+}$]. To allow for detecting small letters in images of limited resolution, the complimentary properties of canny edges and MSER are combined in the edge-enhanced MSER. Further, to generate the stroke width transform image of these regions, the distance transform is applied to efficiently obtain more reliable results.

The geometric as well as stroke width information are then applied to perform filtering and pairing of connected components. Finally, letters are clustered into lines and additional checks are performed to eliminate false positives. The overall process of the text detection is illustrated in Figure 6.2. In comparison to previous text detection approaches, this algorithm offers the following major advantages. First, the edge-enhanced MSER detected in the query image can be used to extract feature descriptors like SIFT and SURF [Low04, BETG08] for visual search. Hence this text detection can be combined with visual search systems without further computational load to detect interest regions. Second, the system provides a reliable binarization for the detected text, which can be passed to OCR for text recognition. Finally, the proposed algorithm is simple and efficient. MSER as well as the distance transform can be very efficiently computed [NS08, Bai04] and determining the stroke width requires only a lookup table (Section 6.1.3).

The flowchart of this text detection algorithm is illustrated in Figure 6.3. At the input of the system, the image intensities are linearly adjusted to enhance the contrast. Subsequently, MSER regions are efficiently extracted from the image [NS08] and enhanced using Canny edges obtained from the original gray-scale image (Section 6.1.1). As a next step, the resulting CCs are filtered using geometric constraints on properties like aspect ratio and number of holes (Section 6.1.2). The stroke width information is robustly computed using a distance transform (Section 6.1.3) and objects with high variation in stroke width are rejected. Text candidates are grouped pairwise and form text lines. Finally, words within a text line are separated, 
giving segmented word patches at the output of the system.

In the following sections the individual steps of this novel text detection algorithm are described. Section 6.1.5 demonstrates the robust performance of the proposed system and Section 6.1.6 summarizes the central properties of this text detector.

\subsubsection{Edge-enhanced Maximally Stable Extremal Regions (EMSER)}

As the intensity contrast of text to its background is typically significant and a uniform intensity or color within every letter can be assumed, MSER is a natural choice for text detection. While MSER has been identified as one of the best region detectors $\mathrm{MTS}^{+} 05$ due to its robustness against view point, scale, and lighting changes, it is sensitive to image blur. Thus, small letters cannot be detected or distinguished in case of motion or defocus blur by applying plain MSER to images of limited resolution. Figure 6.4a shows an example where multiple letters are identified as a single MSER region. To cope with blurred images we propose to combine the complimentary properties of Canny edges [Can86] and MSER. The outline of extremal regions can be enhanced by applying the precisely located but not necessarily connected Canny edges. As shown in Figure 6.4a, the MSER pixels outside the boundary formed by the Canny edges are removed. This is achieved by pruning the MSER along the gradient directions (indicated by the blue arrows) computed from the original grayscale image. A cone with an aperture of $30^{\circ}$ is applied for the pruning. As a rule of thumb, for this approach character need to be at least 10 pixels in height to be detected as such. Since the type of the letter (bright or dark) is known during the MSER detection stage, the gradient directions can be adapted to guarantee that they point towards the background. Figure 6.4b shows the edge-enhanced MSER, which provides a significantly improved representation of the text where individual letters are separated. This not only improves the performance of geometric filtering (Section 6.1.2), but also increases the repeatability of MSER based feature matching under different image blur conditions.

\subsubsection{Geometric Filtering}

With the extraction of edge-enhanced MSER, we obtain a binary image where the foreground CCs are considered as letter candidates. As in most state-of-the-art text detection systems, a set of simple and flexible geometric checks on each CCs is performed to filter out non-text objects. First of all, very large and very small objects are rejected. Then, since most letters have aspect ratio being close to 1 , we reject CCs with very large and very small aspect ratio. A conservative threshold on the aspect ratio is selected to make sure that some elongated letters such as 'i' and 'l' are not discarded. Lastly, objects which contain a large number of holes are eliminated, because CCs with many holes are unlikely to be letter candidates.

\subsubsection{Finding Stroke Width by Distance Transform}

The importance of stroke width information has been emphasized in several recent studies including [SK08, SNDC07, EOW10]. Motivated by Epshtein's work on Stroke Width Transform (SWT) [EOW10, a robust image operator to transform the binary image into its stroke width image is developed. The stroke width image is of the same resolution as the original image, with the stroke width value labeled for every pixel. The stroke width is determined 


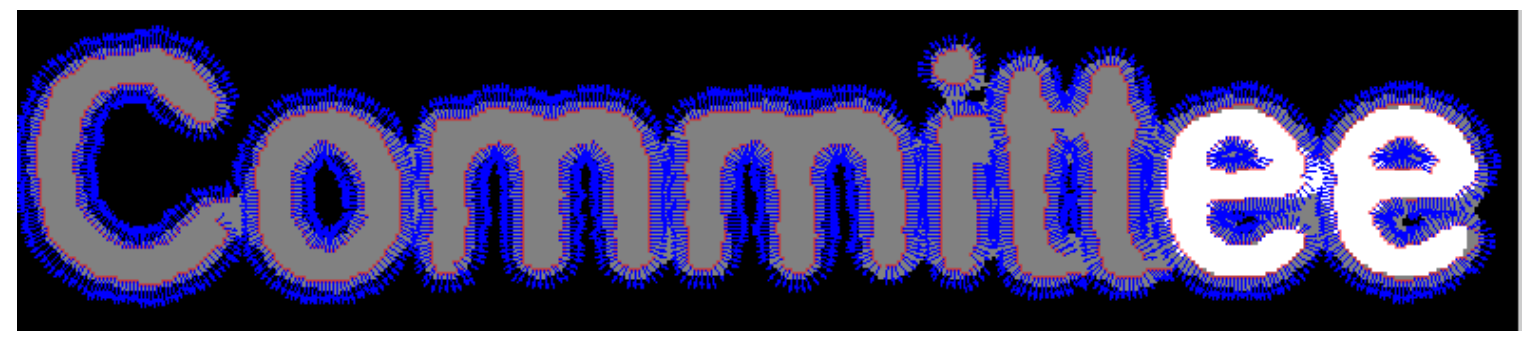

(a)

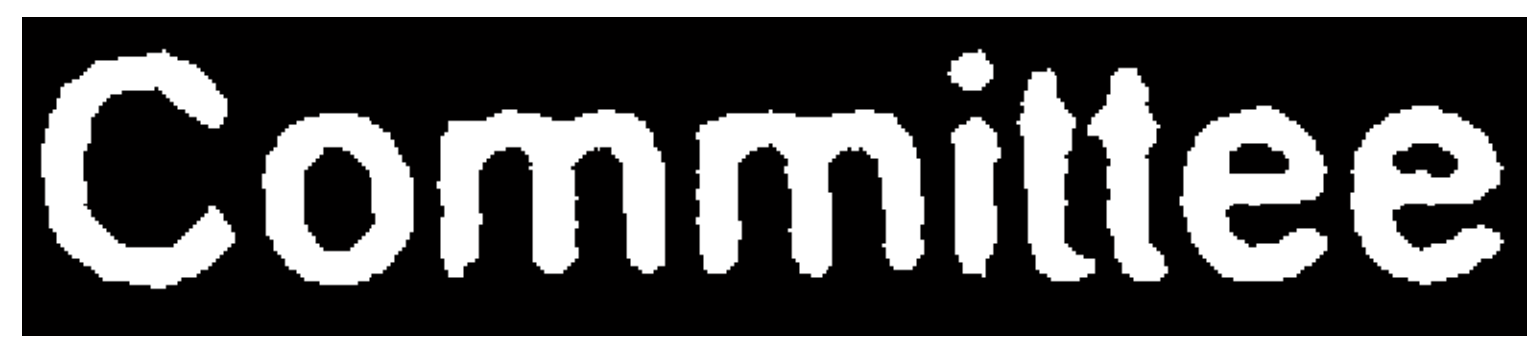

(b)

Figure 6.4: Edge-enhanced MSER. (a) Detected MSER for blurred text. Canny edges are shown in red lines and the blue arrows indicate gradient directions. (b) MSER after pruning along the gradient. (Reproduced from [CTS 11], (C) 2011 IEEE)

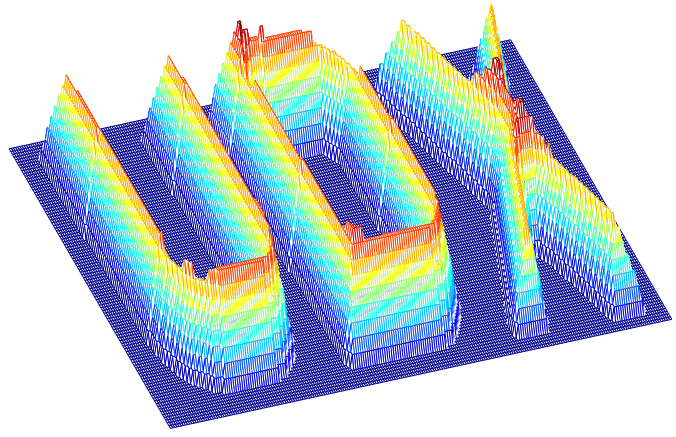

(a) Distance map

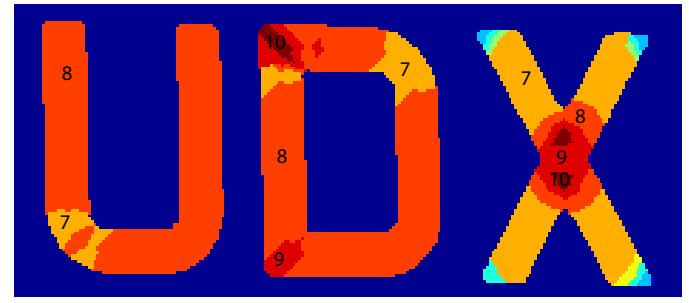

(b) stroke width image

Figure 6.5: Finding the stroke width information. (a) Mesh plot of the distance transformed image. (b) Stroke width image formed by propagating the stroke width information from the ridge to the boundary. The numbers label half of the stroke width since the ridge values correspond to half of the actual width. (Reproduced from [CTS 11], (c) 2011 IEEE) 
using a novel approach based on the distance transform, which is different from the SWT proposed in [EOW10]. Epshtein's SWT forms CCs by shooting rays from the edge pixels along the gradient, and only keeps the rays if they are terminated by another edge pixel having the opposite gradient direction. This method does not work well for complex fonts, as the directed rays cannot recover strokes if the two stroke edges are not in parallel.

Consequently, the stroke width CCs formed by the SWT often have undesirable holes appearing in curved strokes or stroke joints. In contrast to Epshtein's SWT, the method proposed in this section guarantees that the SW information is provided at every pixel of the original $\mathrm{CC}$ with any stroke shape. The Euclidean distance transform is applied to label each foreground pixel with the distance to its nearest background pixel. As can be seen in Figure 6.5a, the ridge values of the distance map correspond to half the width of the stroke. Then, we propagate the stroke width information from the ridge to the boundary of the object, along the "downhill" direction. The stroke width image is shown in Figure 6.5b, Our method bypasses the need to locate ridge pixels by iteratively propagating the stroke width information, starting from the maximum value to the minimum value of the distance map.

The output of the algorithm is an image where each pixel is assigned a value equal to half of the stroke width. Experiments demonstrate the robust stroke width estimation even for complex fonts. Assuming that the stroke width of characters has a low variation, CCs with a large standard deviation are excluded. The rejection criterion is std/mean $>0.5$, which is invariant to scale changes. The threshold is learned from the training set of the ICDAR $2003\left[\mathrm{LPS}^{+}\right.$03] competition database.

\subsubsection{Text Line Formation and Word Separation}

Text lines are important cues for the existence of text, as text almost always appears in the form of straight lines or slight curves. To detect these lines, we first pairwise group the letter candidates using the following rules. As letters belonging to the same text line are assumed to have similar stroke width and character height, two letter candidates are paired if the ratio of their stroke width medians is lower than 1.5 and their height ratio is lower than 2 (taking upper and lower case letters into account). Additionally, two CCs should not be paired if they are very distant.

Subsequently, text lines are formed based on clusters of pairwise connected letter candidates. A straight line is fitted to the centroids of pairs of letter candidates within each cluster and the line that intersects with the largest number of text candidates is accepted. The process is iterated until all text candidates have been assigned to a line, or if there are less than three candidates available within the cluster. A line is declared to be a text line if it contains three or more text objects.

Improbable text lines are filtered out by two additional validation steps. As shown in Figure 6.2b, a false text line is formed along the repetitive windows. Repeating structures such as windows and bricks are commonly seen in urban images, resulting in a large number of false positives. This can be avoided by applying template matching among the letter candidates. A text line is rejected if a significant portion of the objects are repetitive. Also, based on the observation that most letters have low solidity (proportion of the object pixels in the convex hull), a text line is rejected if most of the objects within that line have a very large solidity. 


\section{Conference Centre}

(a)

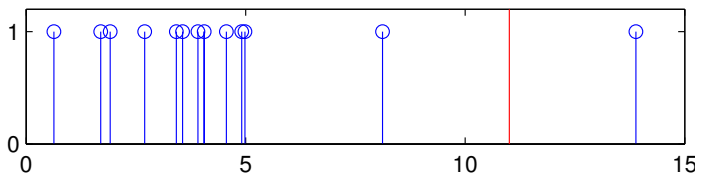

(b)

Figure 6.6: Word separation via binary letter distance classification. (a) The text line is separated into words, based on the vertical projection distances. (b) The distribution of the spacing is classified into two regions using Otsu's method. (Reproduced from [CTS $\left.{ }^{+} 11\right]$, (c) 2011 IEEE)

As a final step, text lines are splitted into individual words by classifying the inter letter distances into two classes: the character spacings and the word spacings. We calculate the distance between the vertical projections of each character along the text line and perform a two class classification using Otsu's method Ots75] as shown in Figure 6.6.

\subsubsection{Experimental Results}

To evaluate this text detection algorithm, it is applied to two different test sets. As a primary test the well-known ICDAR text detection competition data set $\left[\mathrm{LPS}^{+} 03\right.$, Luc05] is used, which was also used as a benchmark for [EOW10, $\mathrm{MTC}^{+}$10, FCM09]. Further, the algorithm is applied to a document database, which has been created to test a document retrieval system based on text as well as low bit rate features in [ $\left.\mathrm{TCC}^{+} 11\right]$. The results are shown in the following subsections.

\section{ICDAR Text Detection}

Two competitions (ICDAR 2003 and 2005) have been held to evaluate the performance of various text detection algorithms $\left.\mathrm{LPS}^{+} 03, \mathrm{Luc05}\right]$. To validate the performance of the proposed system, the metrics defined in [Luc05] are used and the algorithm is run on the ICDAR competition dataset. The precision and recall are defined as $p=\sum_{r_{e} \in E} m\left(r_{e}, T\right) /|E|$ and $r=\sum_{r_{t} \in T} m\left(r_{t}, E\right) /|T|$, where $m(r, R)$ is the best match for a rectangle $r$ in a set of rectangles $R$. $E$ and $T$ are our estimated rectangles and the ground truth rectangles respectively. An $f$ metric is used to combine the precision and recall into one single measure: $f=1 /(\alpha / p+\alpha / r)$, where $\alpha=0.5$ gives equal weights to precision and recall.

Since it is unlikely to produce estimated rectangles which exactly align with the manually labeled ground truth, the $f$ metric can vary from $0.8-1.0$ even when all text is correctly localized.

The text detection performance on the dataset is shown in Table6.1. The results in the lower half include the contestants in $\mathrm{LPS}^{+} 03$, Luc05, where Hinnick Becker's approach achieves the highest $f$ score of 0.62 . The upper half contains the results of the text detection system proposed in this thesis and the state-of-the-art algorithms. The EMSER-based algorithm achieves a $f$ score similar to [EOW10, outperforming all results from the text detection competition. The complexity of the overall detection system is mainly driven by the MSER extraction stage, which is $O(n)$, and requires less than $200 \mathrm{~ms}$ for an image resolution of 


\begin{tabular}{lccc}
\hline \hline Algorithm & precision & recall & $\mathrm{f}$ \\
\cline { 1 - 3 } Our system & $\mathbf{0 . 7 3}$ & 0.60 & $\mathbf{0 . 6 6}$ \\
Epshtein [EOW10] & $\mathbf{0 . 7 3}$ & 0.60 & $\mathbf{0 . 6 6}$ \\
Minetto [MTC ${ }^{+}$10] & 0.63 & 0.61 & 0.61 \\
Fabrizio [FCM09] & 0.46 & 0.39 & 0.43 \\
\hline Hinnerk Becker & 0.62 & $\mathbf{0 . 6 7}$ & 0.62 \\
Alex Chen & 0.60 & 0.60 & 0.58 \\
Ashida & 0.55 & 0.46 & 0.50 \\
HWDavid & 0.44 & 0.46 & 0.45 \\
Wolf & 0.30 & 0.44 & 0.35 \\
Qiang Zhu & 0.33 & 0.40 & 0.33 \\
Jisoo Kim & 0.22 & 0.28 & 0.22 \\
Nobuo Ezaki & 0.18 & 0.36 & 0.22 \\
Todoran & 0.19 & 0.18 & 0.18 \\
Full & 0.10 & 0.06 & 0.08 \\
\hline \hline
\end{tabular}

Table 6.1: Evaluation of text detection algorithms.
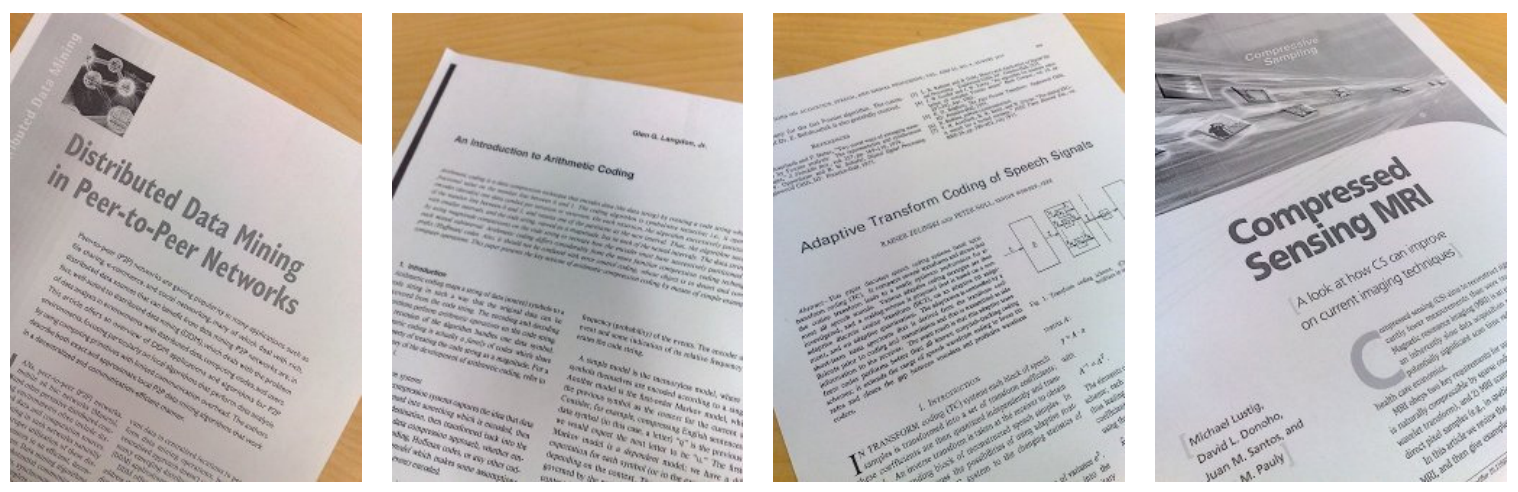

Figure 6.7: Document images under various view points. (Reproduced from [TCC 11$]$, (C) 2011 IEEE)

1280x960 pixels on a $2.5 \mathrm{GHz}$ CPU.

\section{Document Title Text Detection}

As a second test the proposed text detection system is applied to perform a mobile paper search by recognizing the document title in images recorded with a camera-phone and querying databases like Google Scholar in $\left[\mathrm{TCC}^{+} 11\right]$. The first step for such a mobile paper search system is to detect the title text within the document images as shown in Figure 6.7. The performance of the text detection algorithm is evaluated by checking the correctly detected bounding boxes around the title text. We use a stringent criterion and declare a title to be correctly detected only when all letters within the title are detected. Out of 501 SVGA size images, 477 titles were correctly identified, achieving a performance score of $95 \%$. The cases where the detection fails are due to excessive blur and out of focus. 


\subsubsection{Summary}

To exploit text as an important source of information in the context of CBIR, a novel low complexity text detection algorithm is proposed, which employs Maximally Stable Extremal Regions (MSER) as basic letter candidates. To overcome the sensitivity of MSER with respect to image blur and to detect even very small letters (minimum of approximately 10 pixels), an edge-enhanced MSER which exploits the complimentary properties of MSER and Canny edges is developed. Further, a novel image operator to accurately determine the stroke width of binary CCs is presented. The proposed method has demonstrated state-of-the-art performance for localizing text in natural images. The detected text are binarized letter patches, which can be directly used for text recognition purposes. Additionally, the system can be efficiently combined with standard visual search systems by sharing MSER as interest regions. 


\subsection{Visual Phrase based CBIR}

With text being robustly detected in natural images at low complexity, now the information provided has to be efficiently integrated into the contend based image retrieval engine. As described in the motivation of this chapter, the visual appearance of individual characters combined with their spatial order provides a very high amount of distinctiveness, which could allow us to enable visual location recognition even in feature poor environments. Instead of performing a complex and failure prone OCR to convert characters into letters of an alphabet, the unique appearance of characters should be encoded using existing local feature descriptors like SIFT or SURF [Low04, BETG08]. Ideally, the information provided by the combined feature descriptors of a text line, should be seamlessly integrated into existing CBIR systems with minimal increase in computational complexity. In the following subsection related work on exploiting text-related information is discussed.

\subsubsection{Related Work}

Recently, Posner et al. PCN10 proposed a system which allows robots to read writings in natural images and to interpret the content semantically based on recognized text. Images are recorded using a stereo camera mounted on a pan-tilt head on top of the robot. Text is detected and subsequently recognized using the open source software Tesseract as the OCR system [Smi07]. After performing a probabilistic error correction using a dictionary, recognized text is associated to a list of places and views that relate semantically to the search term. The authors state to be the first to exploit text-spotting in natural images in a robotic context.

In the context of document retrieval several approaches have been developed to efficiently and effectively retrieve documents including a queried text body under variations with respect to rotation, resolution, distortion and incompleteness. Most state-of-the-art approaches try to avoid complex and time consuming OCR which would also fail for low resolution query images. In [LYK $\left.{ }^{+} 09\right]$, the authors use SIFT as a detector and an adapted SIFT descriptor with increased distinctiveness for text documents. Retrieval is performed by searching for documents with the largest number of matching feature descriptors using a standard approximate nearest neighbor search.

Li et al. [LFWL09] perform document retrieval using geometric features. To overcome the problem of recognizing the shape of a character at low resolution (required for OCR based methods) they go to higher level features by measuring the relative word length in pixels. A single feature sequence is used to describe a document. Due to the loss in information, comparably long text passages are required to accumulate enough distinctiveness. Unfortunately, reliable word separation turns out to be a tough problem in natural images of, e.g., store signs, etc. Furthermore, approximate matching of the sequences requires time consuming dynamic programming.

Lu et al. [LLT08] use topological shape features including character ascenders, descenders, holes, and water reservoirs to annotate detected words in images with a so called word shape code. Hence, the character set of 62 Roman letters and numbers is transferred to 35 character codes, which are concatenated to a word shape code. While this allows for more robustness against distortions and limited resolution, the extraction of the shape features is time consuming. 


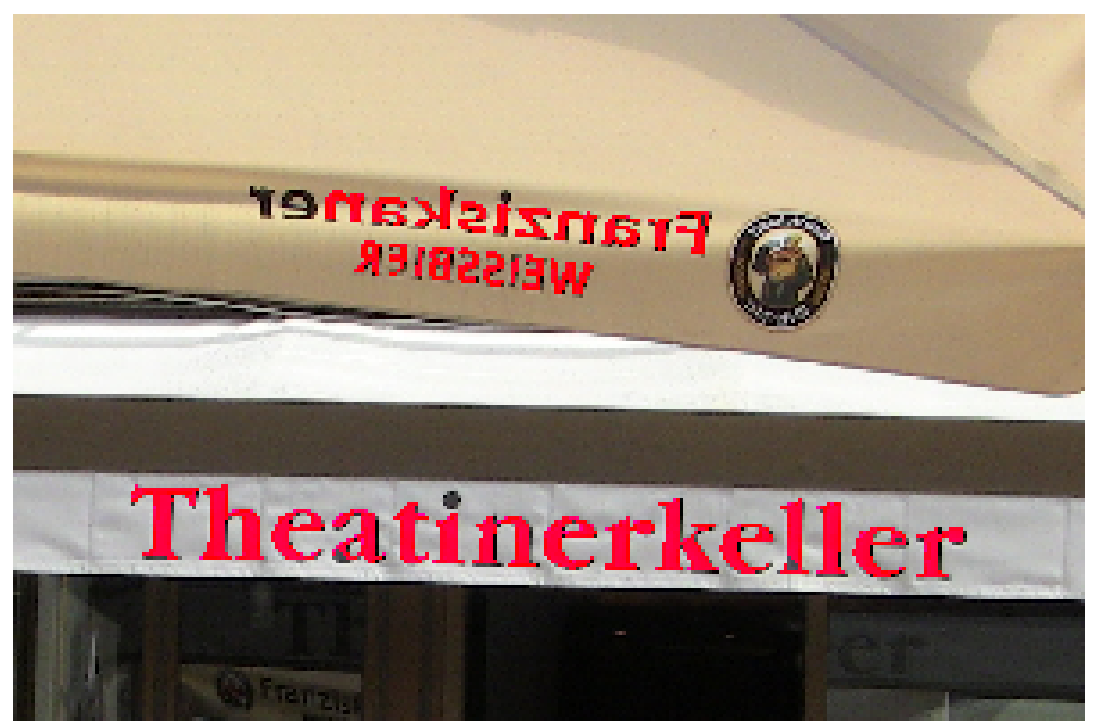

Figure 6.8: Text detection using the EMSER approach described in Section 6.1. Detected character regions are shown in red color. (Reproduced from [SHH 11], (C) 2011 IEEE)

OCR and local feature descriptors are combined in the mobile document retrieval system proposed by Tsai et al. [ $\mathrm{TCC}^{+} 11$. As described above, relevant documents are rapidly retrieved from the web by applying OCR on the larger and distinctive document title and by performing a text search in on-line databases. Local feature descriptors are used for an image based comparison of the returned documents. Fitting an affine model using RANSAC allows for the verification of matched descriptor pairs.

In fact, applying a geometric verification of descriptor matches implicitly enforces the character ordering on a document image and thus significantly increases the performance of the retrieval system. This post processing step, however, only removes false matches and does not increase the distinctiveness of features. Hence, a large number of features is required to reliably fit a model. Further, the fitting process requires significant computational complexity and is only applied to rerank the top results of the retrieval system. To reduce complexity, usually models with fewer degrees of freedom and hence simplified assumption with respect to the projective transformations are applied [ $\left.\mathrm{PCI}^{+} 07\right]$. However, fast geometric reranking approaches like [ $\left.\mathrm{TCC}^{+} 10 \mathrm{~b}\right]$ and the integration of global geometric relations into the retrieval process $\left[\mathrm{CWL}^{+} 10\right.$, LSP06 hardly increase the performance in location recognition, due to the complex 3-dimensional structure of the scenes.

Hence, we need to enforce geometry constraints on local patches where features can be assumed to be on a plane. Wu et al. [WKIS09] bundle SIFT features within MSER regions [MCUP04] for partial-duplicate web image search. Here, the order of the SIFT features along the image $\mathrm{x}$ and $\mathrm{y}$ axes within an MSER is used to enforce a weak geometric verification of a match. While the verification is integrated into the scoring process, every associated database feature has to be individually checked with respect to the order of the feature bundle, which increases computational complexity. This approach relies on the assumption that an MSER region is completely contained within the borders of a single rigid object, which 


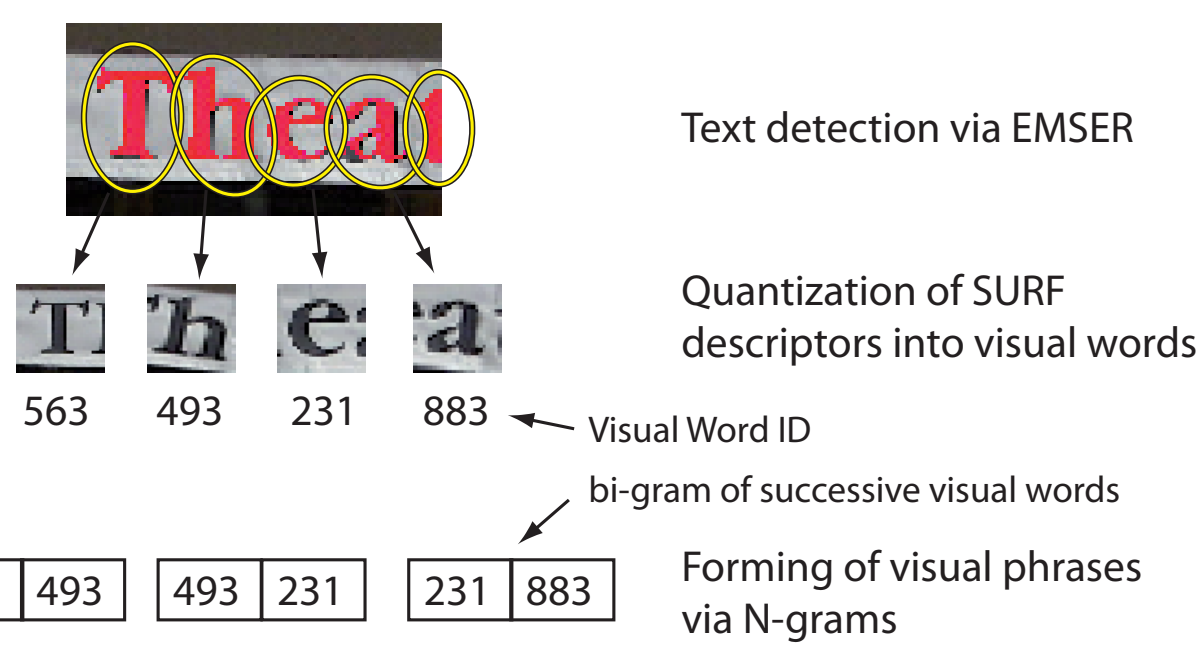

Figure 6.9: Schematic illustration of the text based image retrieval approach. Characters are detected via EMSER and represented using SURF descriptors. A quantization of the descriptors into visual words allows us to assign a visual word ID to each detected character. Strings of characters are represented by subsequences ( $N$ grams) of successive visual word IDs. Image retrieval is performed by identifying those images that include the same $N$-grams using an inverted file structure. (Reproduced from [SHH+11], (C) 2011 IEEE)

can be confirmed for most cases. Only few corners or blobs, however, can be found within the detected region due to the nature of MSER regions (detecting clearly delimited homogeneous regions). Thus, most detected features are found in the outer areas of the detected regions, close to the object boundaries, which leads to a low repeatability of the corresponding descriptors.

\subsubsection{Detecting Visual Phrases}

We propose to improve CBIR by exploiting the coplanarity of characters within writings in natural images. Based on the assumption that detected characters lie on a plane, the visual features of a possibly large area of the scene can be combined to significantly increase the distinctiveness. Further, the distinct gradients of characters provide considerably more information than features describing textures of windows or foliage, which rather impair retrieval performance.

As described above, the first step in our approach is to detect text and individual characters in natural images using the EMSER text detection approach proposed in Section 6.1. In Figure 6.8, detected character regions are drawn in red color on top of the original image. Since no OCR or dictionary is used, also mirrored text (upper writings in Figure 6.8) and false positives can get accepted. However, regions that share very similar geometric properties and are at the same time arranged along a straight line are likely to be found on a planar surface as well.

As a next step, ellipses are fitted around detected character regions. The transformation from the ellipse to a circle is applied to the enclosed texture to normalize the patch and 


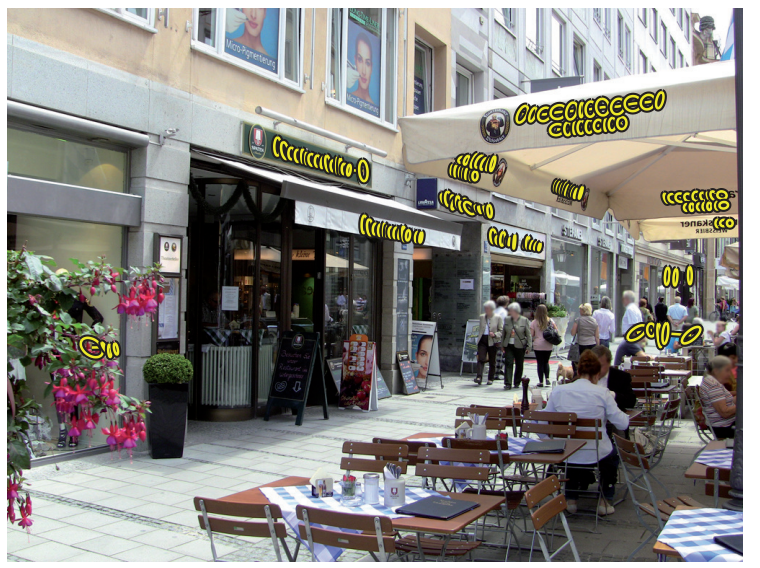

(a)

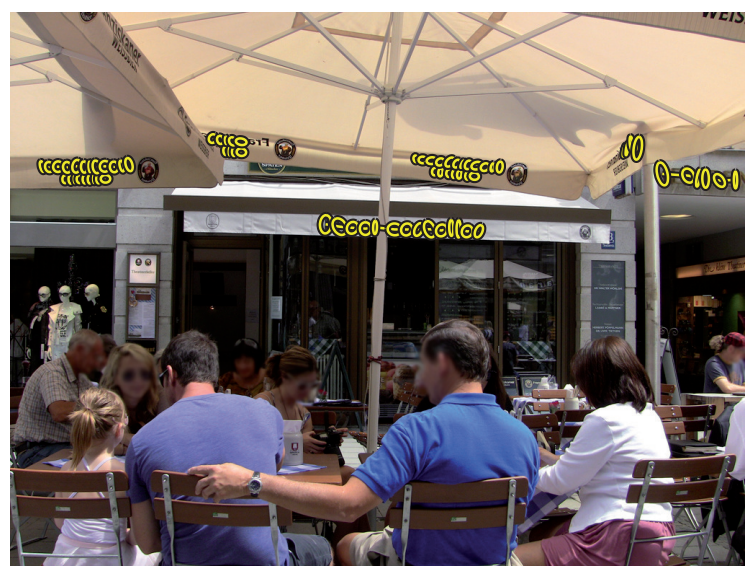

(b)

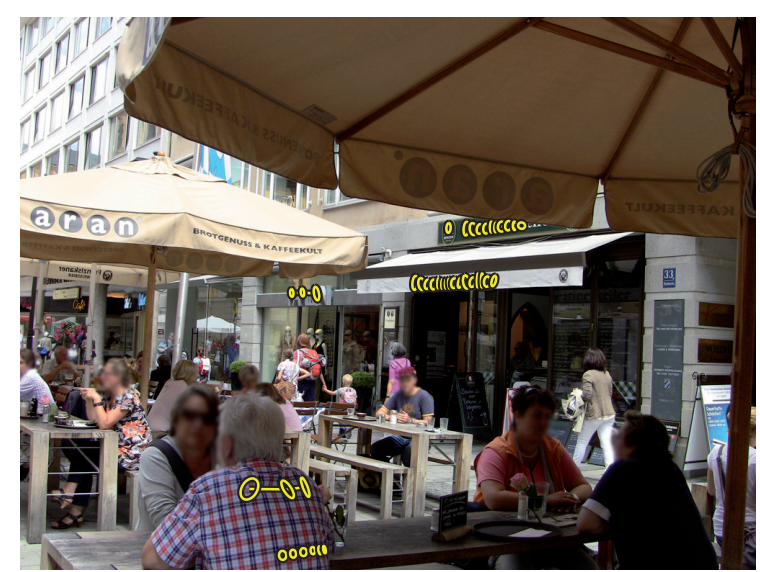

(c)

Figure 6.10: Image recordings in Munich using a camcorder. Despite the presence of the dynamic objects, the complex 3-dimensional scene and the large baselines, the images a-c are reliably matched using the proposed text based image retrieval engine. Detected letters are highlighted by yellow ellipses. (Reproduced from [SHH 11], (c) 2011 IEEE)

thus increase the robustness against affine transformations. As shown in Figure 6.9, the size of the ellipse is multiplied by a constant factor such that additional background is included but can be safely assumed to be on the same plane. As a particular character shape can appear at different locations, the background pattern extends the distinctiveness. To describe the appearance of the characters, SURF [BETG08] descriptors are extracted on each of the patches. To be robust against in-plane rotations, the rotation of the patches is determined by the detected baseline. By replacing the standard orientation assignment of SURF based on the dominant gradient within a patch, in-plane rotation invariance is removed resulting in an efficient increase in the distinctiveness of the descriptor. 


\subsubsection{Visual Phrase based Retrieval}

In order to be able to efficiently deal with large databases, Sivic and Zisserman [SZ03] proposed to reformulate the CBIR problem into a text retrieval problem by clustering descriptors into so called visual words with the aid of the $k$-means algorithm. At fine quantization, descriptors associated with a visual word follow a texture pattern that is sufficiently represented by their mean (see Section 2.2.2). A pairwise comparison between query and database descriptors can be avoided as images including the same visual word can be efficiently determined within an inverted file structure. As the spatial layout of features within query and database image is ignored in the matching process, this approach is called Bag-of-Visual-Words or Bag-ofFeatures (BoF). In Section 2.2.2 the so called approximate $k$-means (AKM) [PCI ${ }^{+} 07$ ], which accelerates the assignment of a descriptor to a visual word via approximate nearest neighbor search, is shown to perform well when matching images at wide baselines and can be flexibly adjusted with respect to the query time.

In state-of-the-art BoF based approaches, a vocabulary of about one million visual words is used to achieve sufficient distinctiveness per descriptor to differentiate the database images [ $\mathrm{PCI}^{+} 07$ ], each typically represented by about 1000 visual words. Due to this large vocabulary and descriptor variations caused by different viewing directions, only about $10 \%$ of the visual words of two corresponding images are actually matched.

In contrast to using all features to differentiate the database images (including frequent textures like windows or foliage), in the proposed visual phrase based approach only the distinctive features on text patterns, i.e., about 30 features per image are exploited. This allows us to reduce the size of the database significantly, i.e., by two orders of magnitude, and to use a vocabulary in the range of only about 600 visual words in total. These small vocabulary sizes are sufficient when combining visual words into visual phrases as will be explained in the following. This considerably increases the chance that two matching descriptors are successfully assigned to the same visual word and reduces the computational complexity of the feature quantization on the mobile device drastically.

As with standard BoF based CBIR systems, the descriptor of each letter region is quantized to a visual word using the approximate $k$-means approach $\left[\mathrm{PCI}^{+} 07\right]$ to transform each writing in a natural image into a string of visual words. Now, the retrieval of relevant images via visual words is shifted to searching for images that include the same visual phrases.

The distinctiveness of a single visual phrase grows with the number $S^{N}$ of possible phrases of the same length. Here, $S$ is the size of the vocabulary and $N$ is the number of visual words in the string. While the distinctiveness increases exponentially with the string length, the probability that not a single quantization error, i.e., quantization into the wrong visual word, occurs decays exponentially with the length $\left(p_{q}{ }^{N}\right.$, with $p_{q}$ as the probability of correct quantization).

Figures 6.11, 6.12, 6.13, show image patches of five distinct visual words for a 10, 100, and 1000 -word visual vocabulary. Clearly, with increasing vocabulary size, the variance within a visual word is reduced and thus distinctiveness is increased. However, with large vocabulary sizes, also the probability of correctly assigning a feature descriptor to a visual word decreases. Thus, the probability of correct quantization of individual features is a function $p_{q}(S)$ that decays with the vocabulary size $S$. While the probability of correct quantization $p_{q}$ is high due to the small vocabulary, additionally all $N$ characters have to be detected $\left(p_{d}{ }^{N}\right.$, with $p_{d}$ as the probability of correct text detection) to allow for an exact match between the visual 


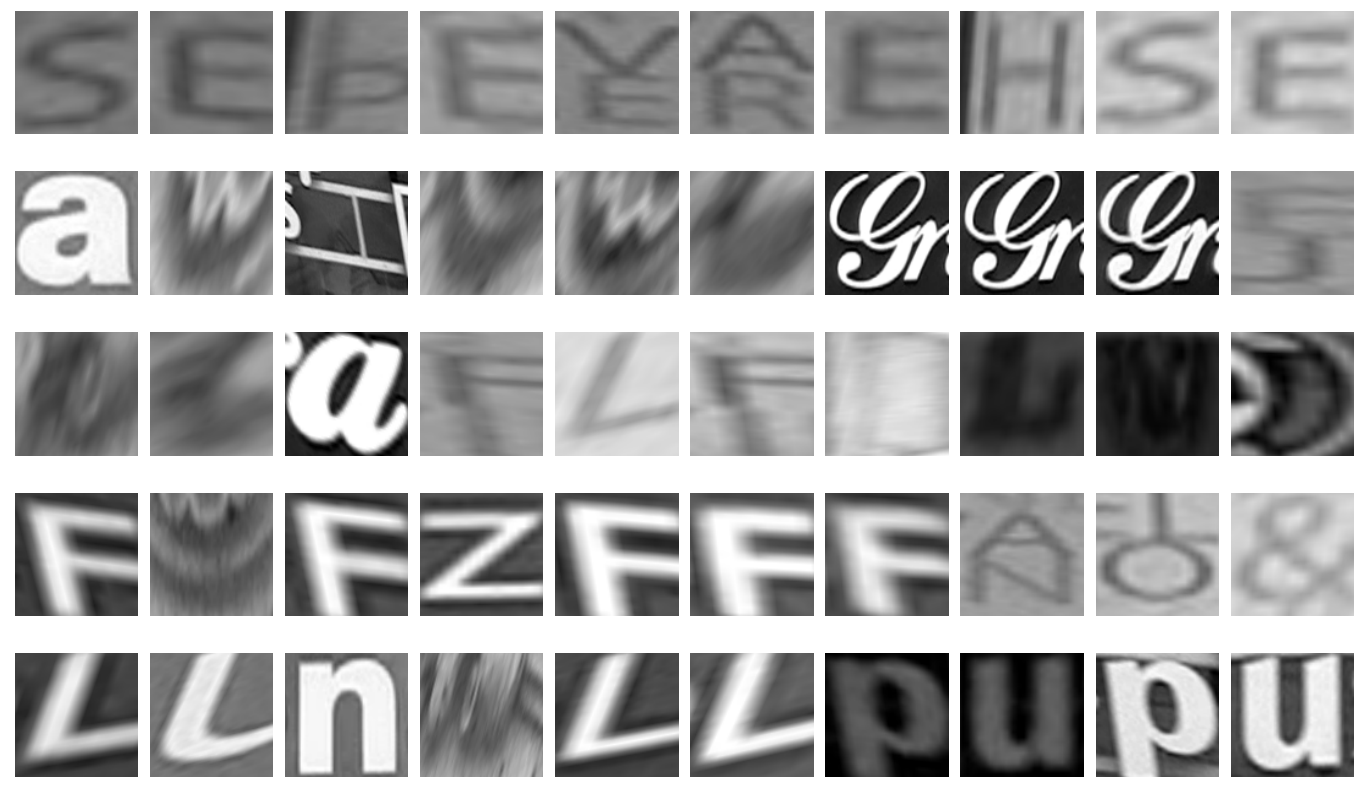

Figure 6.11: Sample image patches of five distinct visual words for a 10-word visual vocabulary. Each row represents a different visual word category.

phrases in the database and query images. Thus, the probability that a visual phrase is correctly detected and all letters are correctly quantized is given as:

$$
p_{c}=p_{d}^{N} \cdot\left(p_{q}(S)\right)^{N}
$$

Due to the imperfections in the detection and quantization process we face three typical types of errors when matching visual phrases. A mismatch (also termed substitution) occurs when a letter was (in part) detected but assigned to a different visual word. Insertions or deletions are provoked by incorrect text detection. Deviating character detection results between the query and the reference can have multiple causes including occlusions by foreground or dynamic objects, defocus or strong motion blur, or simply only a detail of the reference view was recorded.

To cope with these imperfections an approximate matching has to be performed. Being a fundamental problem in a large range of applications, numerous approaches to compute string similarity have been developed and can be characterized as either edit-based or tokenbased. The former rely on computing the minimum number of edit-operations that distinguish strings from each other. Very frequently used is the Levenshtein-Distance where a valid edit-operation is the substitution, deletion, or insertion of a single character [Lev66]. Other distance measures are, for instance, the Damerau-Distance where also transpositions, i.e., swapping of two adjacent characters, belong to the set of valid operations [Dam64, and the well known Hamming-Distance which considers substitutions only Ham50]. Edit-based methods usually lead to dynamic programming which is time consuming and does not easily allow for acceleration through preprocessing Ukk92.

Token-based approaches measure how much strings have in common. Tokens may be chosen as words, phrases, or N-grams, i.e., substrings of fixed length $N$ [Sha48, Ull77]. While words 


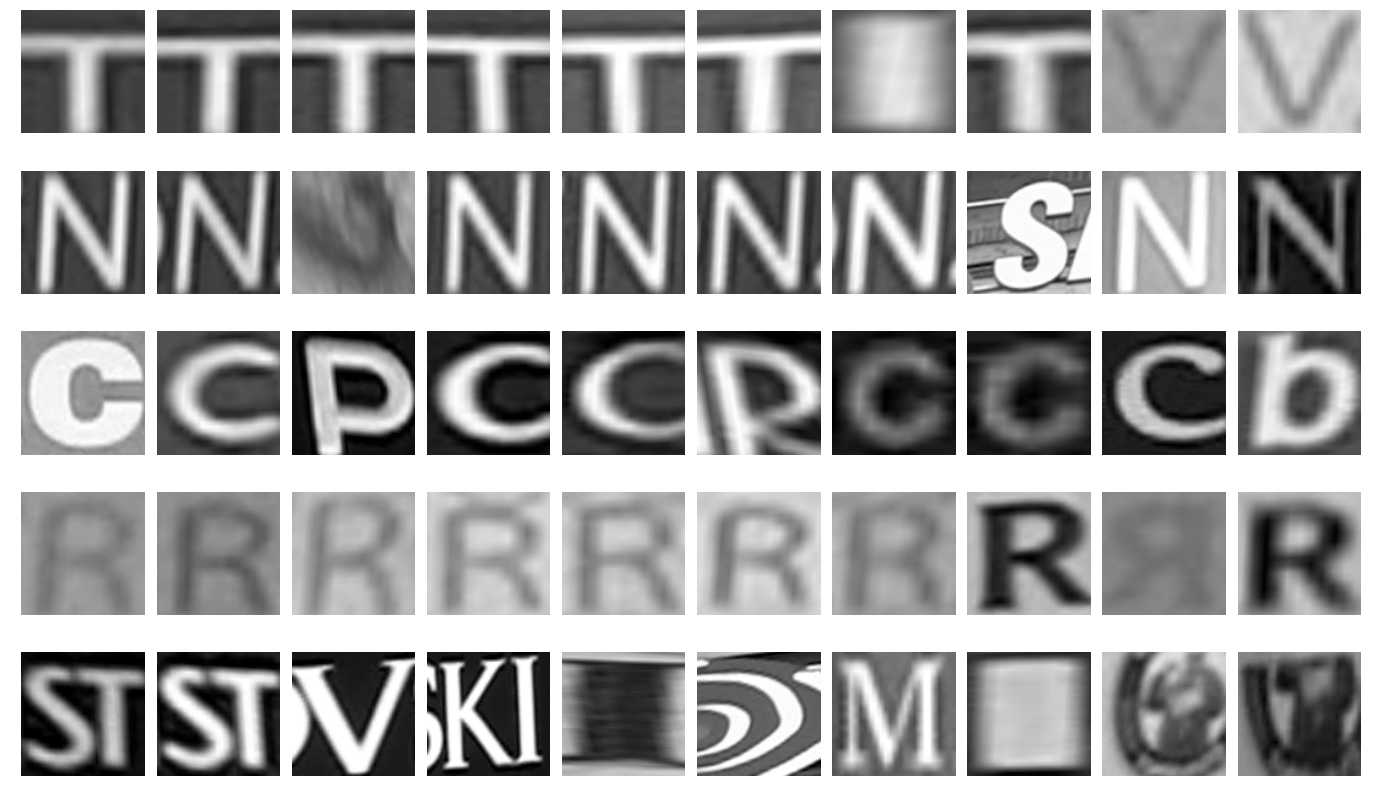

Figure 6.12: Sample image patches of five distinct visual words for a 100-word visual vocabulary. Each row represents a different visual word category.

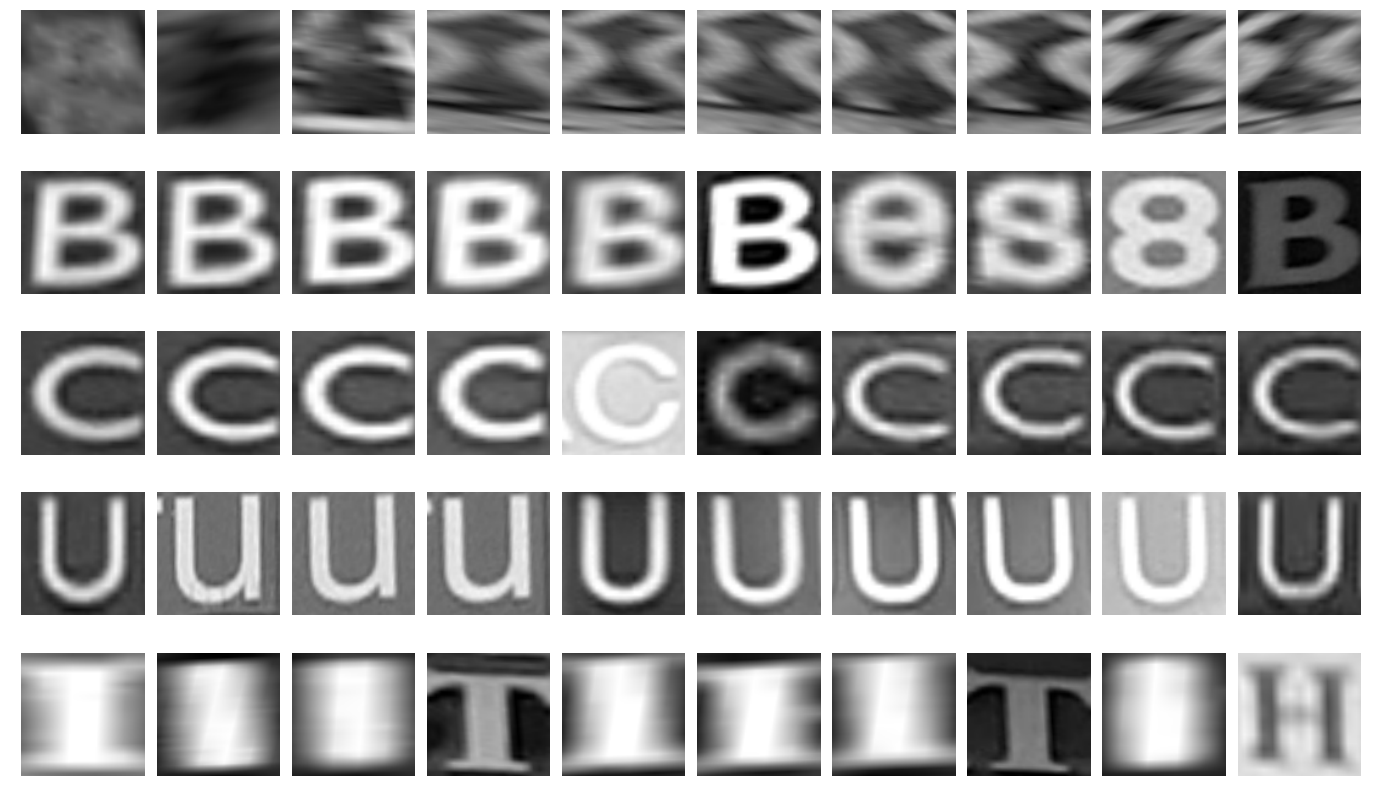

Figure 6.13: Sample image patches of five distinct visual words for a 1000-word visual vocabulary. Each row represents a different visual word category. 


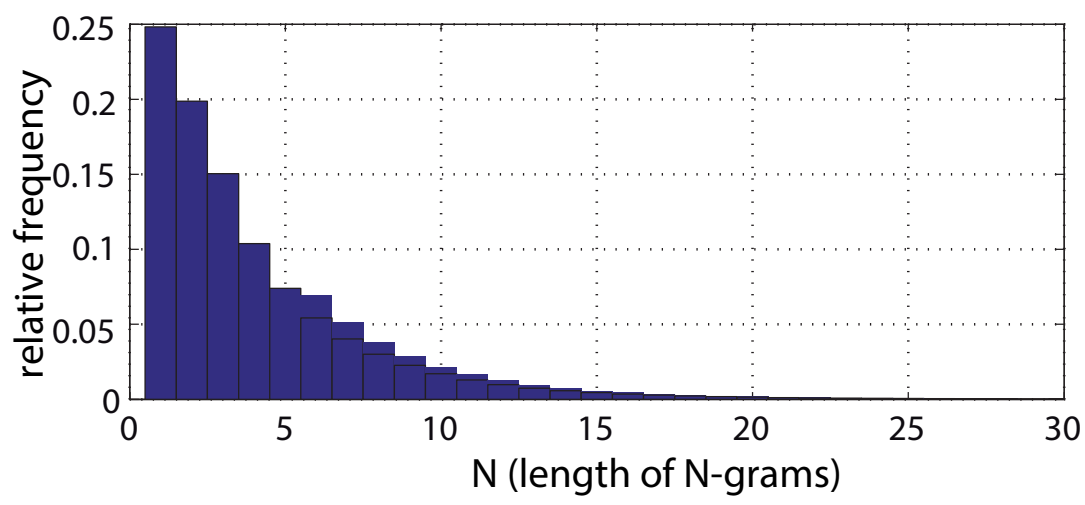

Figure 6.14: Relative $N$-gram occurrence frequencies in the dataset recorded in downtown Munich. (Reproduced from [SHH 11$]$, (c) 2011 IEEE)

and phrases are natural choices, the widely used N-grams offer increased flexibility since they are language independent [NST05], which is a vital property for applications where no well-defined concept of a word exists. Token-based approaches accumulate the number of (exactly) matching tokens between strings and, due to their discrete nature, are well suited for set-theoretic similarity measures which can be computed very efficiently. Examples are the Jaccard-coefficient, Dice's coefficient, the overlap-coefficient (also Simpson-coefficient), and the cosine-similarity [RL08]. The decomposition of a string into tokens does not depend on any queries and so a large amount of computation can be carried out during preprocessing of the data. Moreover, the actual pattern matching step is reduced from approximate to exact matching for which optimal retrieval structures providing constant time complexity already exist. In particular, if the set of features representing a query is sparse compared to the set of features that are present in the database, an inverted index achieves significant reduction of index lookups and thus unmatched performance.

Hence, a string of visual words is represented by its corresponding N-grams as shown in Figure 6.9 (bi-grams in this example). For each, the inverted file stores references to all images in which they occur. Considering a vocabulary of about 500 visual words and trigrams as visual phrases, the set of distinguishable features amounts to 125 million visual phrases, which is large compared to the 1 million visual words in state-of-the-art BoF based approaches.

The retrieval score for the images referenced by the visual phrases occurring in the query is incrementally computed using the Dice's-coefficient $s_{D}$ as shown in Equation 6.2. The number of matched $\mathrm{N}$-grams $M$ is normalized by the sum of $\mathrm{N}$-grams occurring in the query $N_{q}$ and the reference image $N_{r}$. This allows us to cope with databases where the amount of writings differs significantly between the database images.

$$
s_{D}=\frac{2 M}{N_{q}+N_{r}}
$$




\subsubsection{Information Theoretic Model}

As we represent strings of visual words by their substrings, i.e., N-grams, we are interested in the optimal length $N$ and size $S$ of the used visual vocabulary. To avoid determining these parameters via tedious experiments and to understand the N-gram-based matching between a query and a reference image in more depth, we require a measure to estimate the retrieval performance at a given parameter set. Modeling the matching between a query and a reference image as a communication channel where the quantization and detection processes introduce errors, the mutual information expressed in Equation 6.12 describes the dependence between the visual phrases in the query and corresponding reference image. To achieve the best possible performance of the retrieval, the dependence and thus the mutual information should be maximized. The channel source draws symbols from the finite domain of possible visual phrases (indexed by $i=1, \ldots, S^{N}$ ). The probability in Equation 6.1 for correct extraction of an $\mathrm{N}$-gram defines the symbol independent transmission probability that symbol $y=i$ is received given that symbol $x=i$ was sent by the source:

$$
p(y=i \mid x=i)=p_{c}=p_{d}^{N} \cdot p_{q}(S)^{N}=f(S, N)
$$

In simplified notation:

$$
p(y=x \mid x)=p_{c}
$$

For determining the channel capacity, i.e., the optimal correlation between source and receiver, the mutual information needs to be maximized. The optimization parameters in this respect are the visual vocabulary size, $S$, and the visual phrase length, $N$, as these impact the transmission probability.

\section{Mutual Information}

Let $\mathcal{X}=\left\{i \mid i=1, \ldots, S^{N}\right\}$ be the domain of source symbols, and $\mathcal{Y}=\left\{i \mid i=1, \ldots, S^{N}\right\}$ be the domain of receivable symbols. Then the mutual information which describes the dependence between two visual phrases, $x$ and $y$, in a query and a reference image, respectively, is defined as

$$
I_{v p}=\sum_{\substack{x \in \mathcal{X} \\ \mathcal{X}=\left\{i \mid i=1, \ldots, S^{N}\right\}}} \sum_{\substack{y \in \mathcal{Y} \\ \mathcal{Y}=\left\{i \mid i=1, \ldots, S^{N}\right\}}} p(x, y) \cdot \log _{2} \frac{p(x, y)}{p(x) \cdot p(y)}
$$

Using the identity $p(x, y)=p(x) \cdot p(y \mid x)$, this can be written as

$$
\begin{aligned}
I_{v p} & =\sum_{x \in \mathcal{X}} \sum_{y \in \mathcal{Y}} p(x) \cdot p(y \mid x) \cdot \log _{2} \frac{p(y \mid x)}{p(y)} \\
& =\sum_{x \in \mathcal{X}} p(x) \cdot \sum_{y \in \mathcal{Y}} p(y \mid x) \cdot \log _{2} \frac{p(y \mid x)}{p(y)} .
\end{aligned}
$$


Dividing the sum over all possible symbols in $\mathcal{Y}$ into a correct and an incorrect quantization part, Equation 6.7 can be expanded as

$$
I_{v p}=\sum_{x \in \mathcal{X}} p(x) \cdot\left[p(y=x \mid x) \cdot \log _{2} \frac{p(y=x \mid x)}{p(y=x)}+p(y \neq x \mid x) \cdot \log _{2} \frac{p(y \neq x \mid x)}{p(y \neq x)}\right] .
$$

Inserting Equation 6.1 into Equation 6.8 and assuming a uniform distribution of source symbols $\left(p(x)=\frac{1}{S^{N}}\right)$, this becomes

$$
I_{v p}=\sum_{x \in \mathcal{X}} \frac{1}{S^{N}} \cdot\left[p_{c} \cdot \log _{2} \frac{p_{c}}{\frac{1}{S^{N}}}+\left(1-p_{c}\right) \cdot \log _{2} \frac{\left(1-p_{c}\right)}{\frac{S^{N}-1}{S^{N}}}\right] .
$$

There are $S^{N}$ possible symbols for $x$ and the transmission probability is modeled symbol independent, so the remaining sum in Equation 6.9 can be further expanded as

$$
\begin{aligned}
I_{v p} & =S^{N} \cdot \frac{1}{S^{N}} \cdot\left[p_{c} \cdot \log _{2} \frac{p_{c}}{\frac{1}{S^{N}}}+\left(1-p_{c}\right) \cdot \log _{2} \frac{\left(1-p_{c}\right)}{\frac{S^{N}-1}{S^{N}}}\right] \\
& =p_{c} \cdot \log _{2} \frac{p_{c}}{\frac{1}{S^{N}}}+\left(1-p_{c}\right) \cdot \log _{2} \frac{\left(1-p_{c}\right)}{\frac{S^{N}-1}{S^{N}}} .
\end{aligned}
$$

Finally, approximating $\frac{S^{N}-1}{S^{N}} \approx 1$ for large $S^{N}$, Equation 6.11 becomes

$$
I_{v p} \approx p_{c} \cdot \log _{2}\left(p_{c} \cdot S^{N}\right)+\left(1-p_{c}\right) \cdot \log _{2}\left(1-p_{c}\right) .
$$

As becomes evident, the expected information provided by a detected $\mathrm{N}$-gram is determined by its distinctiveness, i.e., the number of possible phrases, $S^{N}$, and the probability $p_{c}$ that a visual phrase is correctly quantized and detected. While the distinctiveness increases with vocabulary size $S$ and N-gram length $N$, the probability of correct detection decreases as shown in Equation 6.3 .

In order to use the result in Equation 6.12 for determining optimal values for $S$ and $N$, a model of the probability of correct quantization $p_{q}(S)$ is required. Up to now, a basic model $p_{q}(S)=\frac{\alpha}{S-\beta}$ is applied where the parameters $\alpha$ and $\beta$ have to be derived from training data and are distinct to a given quantizer. More elaborate models could be established using a reasonably large database with ground truth which could be addressed in future work.

In Figure 6.15 the mutual information is plotted against the vocabulary size $S$ and the $\mathrm{N}$-gram length $N$. In this example, the detection probability $p_{d}$ is assumed to be 0.7 and the parameters $\alpha$ and $\beta$ of the quantization probability function $p_{q}(S)$ are defined by the constraints $p_{q}(1)=1$ and $p_{q}(1 e 6)=0.05$. Thus, we assume that a feature is quantized to the correct visual word with a probability of $5 \%$ when using a vocabulary size of 1 million visual words. These values have been learned using a ground truth dataset and result in a maximum mutual information of 8.22 at $N=3$ and $S=800$.

Further, not only the information obtained from a single N-gram should be considered to find the optimal length $N$ but also the average number of available $\mathrm{N}$-grams per image has to 
be taken into account. Clearly, not every image includes N-grams of length 10, for instance, as shown in Figure 6.14. This N-gram distribution has been generated from image recordings in downtown Munich (example images are shown in Figure 6.10), which will be explained in detail in Section 6.3. To determine the average information per image, the information per $\mathrm{N}$-gram has to be weighted with the expected number of N-grams of a specific length.

\subsubsection{Combined N-grams}

However, it is actually not necessary to constrain the approach to a single N-gram length. Rather multiple N-grams should be combined to exploit all information available. While short visual words, e.g., uni-grams, and bi-grams lead to a high recall, i.e., most of the relevant documents are within the top ranked results, precision may be low since non relevant documents could include the features of the query image and are also listed among the top results. This is typically the case in large databases where the distinctiveness of individual features may be insufficient. N-grams of larger length are less likely to be correctly detected and thus result in a lower recall but provide a higher precision due to their increased distinctiveness. Combining these complimentary properties can result in improved performance in large databases, as will be shown in Section 6.3.

The contributions of each type of N-gram should be weighted according to the information which they are expected to provide. This is achieved by utilizing the mutual information (see Equation 6.12 weighted with the expected number of the respective N-grams. The weighting is integrated into the Dice's-coefficient (see Equation 6.2 as shown in Equation 6.13 providing a combined score $s_{w}$ of multiple N-grams (see Figure 6.14). Here, $w_{i}$ corresponds to the weight of an N-gram of length $i$ and $M_{i}$ is the number of matched $\mathrm{N}$-grams, which is normalized by the sum of $\mathrm{N}$-grams occurring in the query $N_{q}, i$ and the reference image $N_{r}, i$. The score is

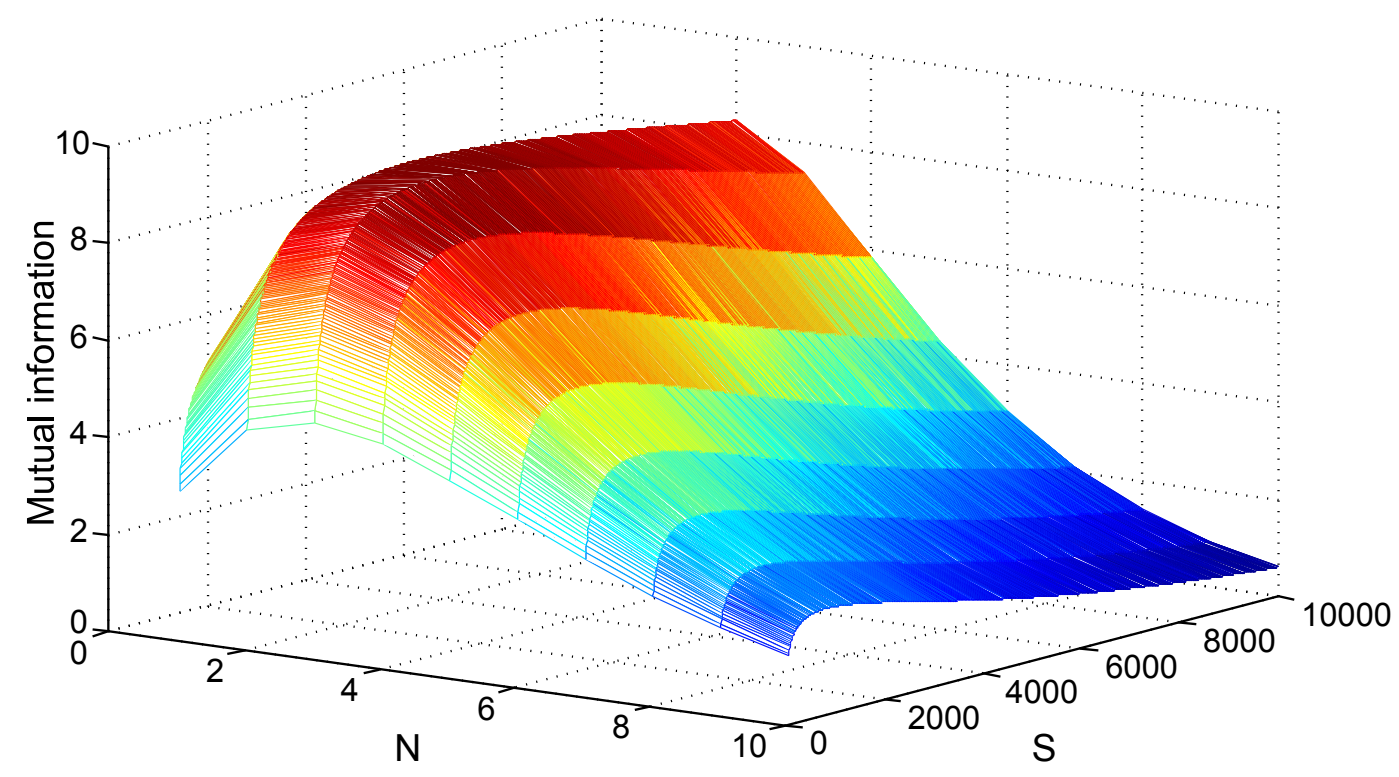

Figure 6.15: Exemplary plot of the mutual information against vocabulary size $S$ and $N$ gram length $N$. 
incrementally computed using an inverted file structure that includes the employed N-gram types.

$$
s_{w}=\frac{2\left(w_{1} M_{1}+w_{2} M_{2}+\ldots\right)}{w_{1} N_{q, 1}+w_{2} N_{q, 2}+w_{1} N_{r, 1}+w_{2} N_{r, 2}+\ldots}
$$

In future work, gapped N-grams should be integrated, which are reported to significantly increase the performance in the context of approximate string matching [BK03]. Here, single characters are skipped to increase robustness against scattered errors. 


\subsection{Experimental evaluation}

In this section, the retrieval performance of the proposed approach is evaluated with respect to four different aspects. In a first experiment, the approach is applied to a large scale outdoor location recognition scenario where query images have to be matched to the closest reference image in an area of approximately $4 \mathrm{~km}^{2}$. The second experiment evaluates the robustness against clutter and occlusions. Query images are matched to reference images that have been recorded along a $5 \mathrm{~km}$ long track in downtown Munich. The scalability of the approach is tested using the Amazon Book Cover dataset, which contains an overall of 203000 images. To demonstrate the advantages of this visual phrase based approach in the context of indoor localization, a door sign recognition system is designed and evaluated.
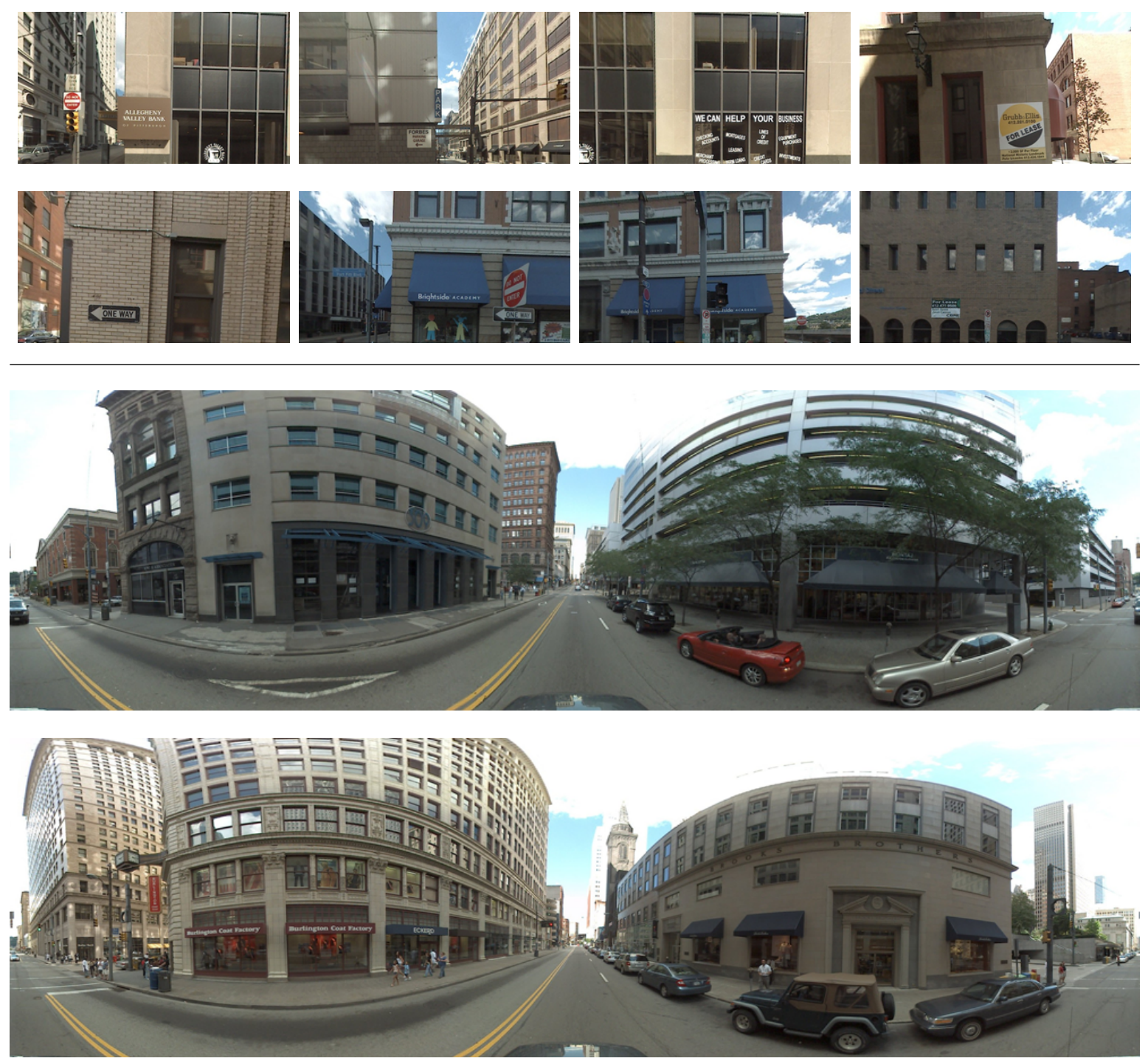

Figure 6.16: Random samples from the Pittsburgh Street View dataset. The two top rows show query images, the two bottom rows database panoramas. 


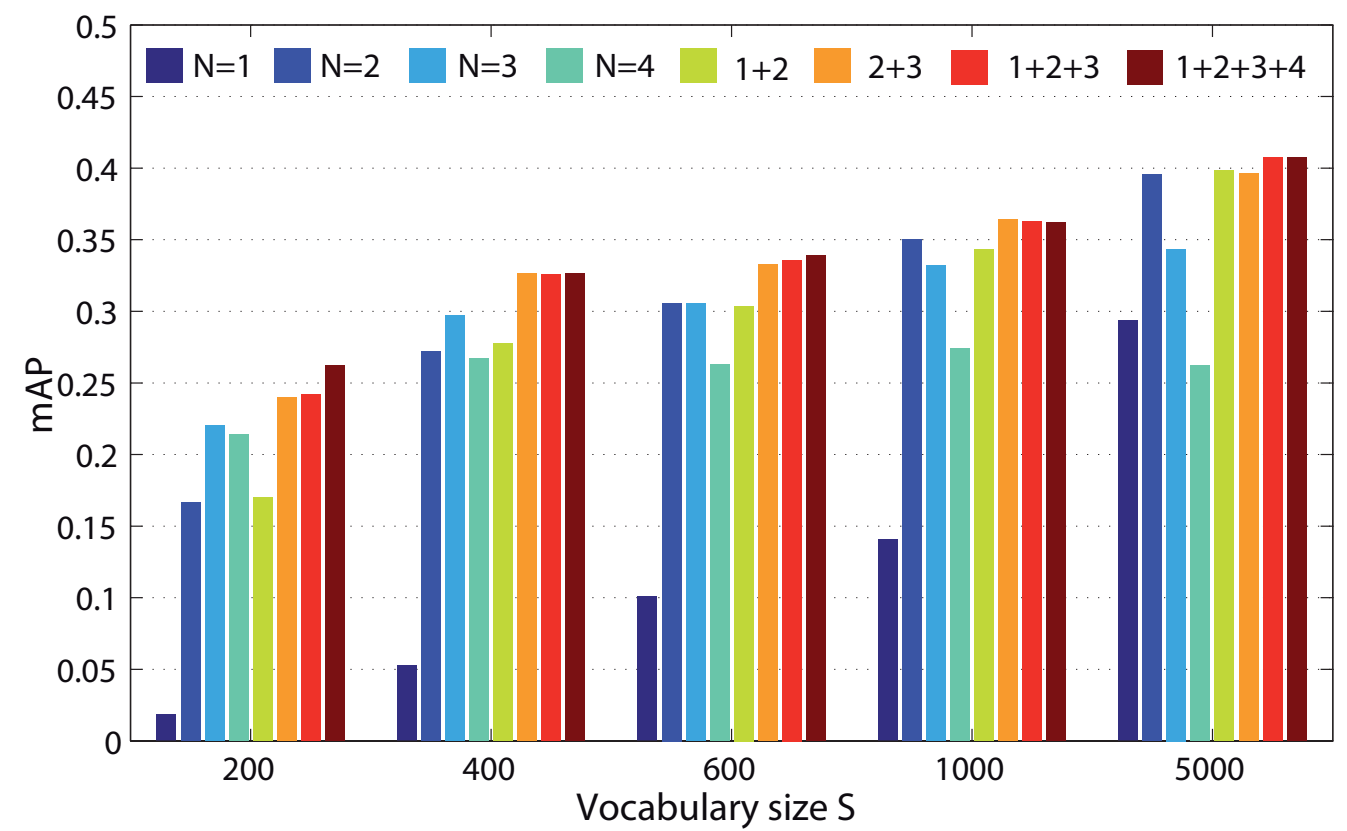

Figure 6.17: $m A P$ score of selected $N$-gram configurations at varying vocabulary size $S$ using the Pittsburgh Street View dataset. (Reproduced from [SHH 11], C) 2011 IEEE)

While the approach can be easily combined with plain BoF based retrieval systems to cope with databases where writings are only occasionally included, we consider only text-related features to illustrate the specific properties of this approach. As evaluation metric the widely used mean average precision (mAP) score is again employed. To recapitulate, the mAP can be interpreted as the area under the precision recall curve and peaks at 1.

In the first experiment, a Google Street View database of downtown Pittsburgh covering an area of approximately $4 \mathrm{~km}^{2}$ is employed, where panoramas are recorded every $2 \mathrm{~m}$ on average. Two rectified images are extracted from each $360^{\circ}$ panorama heading $90^{\circ}$ to the left and right. The resolution of these reference images is limited $(800 \times 600$ pixels) and in several images only few and tiny characters are visible. Query images are recorded at 104 locations that include at least some kind of visible writing as shown in Figure 6.16. To achieve an $\mathrm{mAP}$ of 1 , all reference images within a given radius around the query location have to be ranked first in the result list. By evaluating the approach with this tough retrieval problem the system can be pushed to the limits to illustrate its strengths and weaknesses.

As part of the retrieval process we extract on average 32 text related features per image, which is about two orders of magnitude less then the number of features used in state-ofthe-art BoF-based retrieval systems. As described above, this not only significantly reduces memory requirements of the database but also allows us to employ much smaller vocabularies. In Figure 6.17, the mAP scores for the individual N-gram configurations are plotted against the size of the used visual vocabulary. All images within 6 meters around the query locations have to be retrieved. The first four bars at the respective vocabulary sizes show the performance of uni-, bi-, tri-, and four-grams. Further, four combined N-gram configurations are evaluated as described in the legend of the figure. A significant performance 


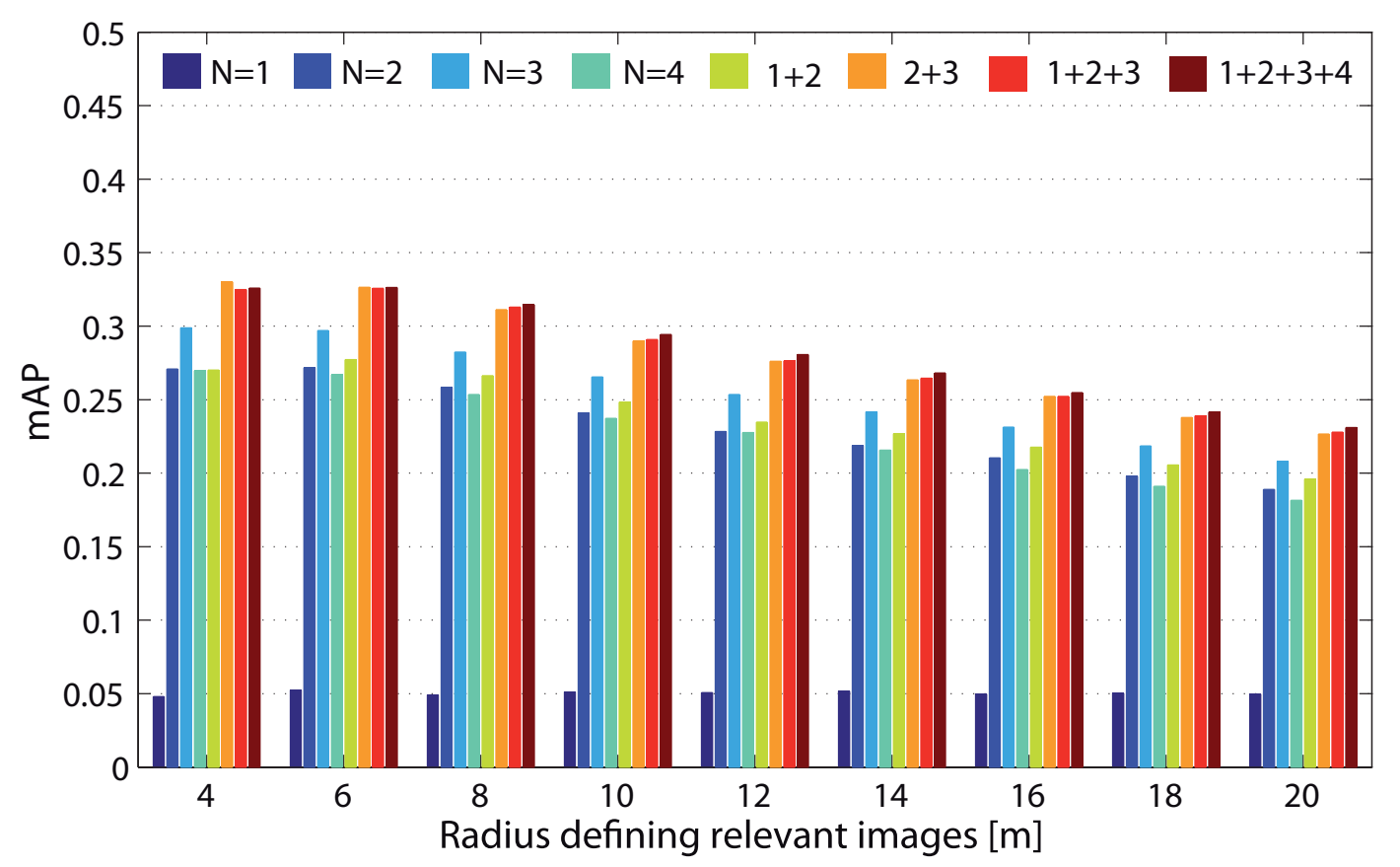

Figure 6.18: $m A P$ score of selected $N$-gram configurations at vocabulary size 400 and varying radius defining the relevant images in the Pittsburgh Street View dataset. (Reproduced from [SHH 11], (c) 2011 IEEE)

increase is observed when comparing uni-grams with bi-grams at all vocabulary sizes, which is explained by the enhanced distinctiveness of the latter. While tri- and four-grams can outperform bi-grams at low vocabulary sizes, their mutual information begins to degrade at higher values of $S$ due to the decreasing probability of correct quantization. The best overall performance of single $\mathrm{N}$-grams is achieved at 5000 visual words by bi-grams. Increasing the visual vocabulary further to 10000 visual words results in a reduced or stagnant performance at all N-grams. Even though characters on store signs are usually very individual, it is hard to reliably distinguish more than 10000 different character descriptors in an affine invariant manner. Thus, increasing the visual vocabulary further results in over quantization, which impairs the performance.

The approach is compared to plain BoF-based CBIR applied to regular MSERs, where we extract approximately twice the number of features (67 on average). The features are selected with respect to the variance of the MSERs to choose those with the highest repeatability. The best performance is achieved at a vocabulary size of 15000 visual words resulting in an mAP of 0.251. As no higher level selection of the features is applied, features are possibly detected on dynamic objects and object borders impairing the performance. In comparison, the unigrams in Figure 6.17 consider exclusively text related features, attaining a maximum mAP of 0.29 already for a vocabulary size of 5000 visual words.

Reducing the size of the inverted file, the time required to quantize features with smaller vocabularies, as well as the number of features to represent an image, the proposed approach provides beneficial properties for several image retrieval applications. As the size of the inverted file scales linearly with the number of features in the database, the memory require- 

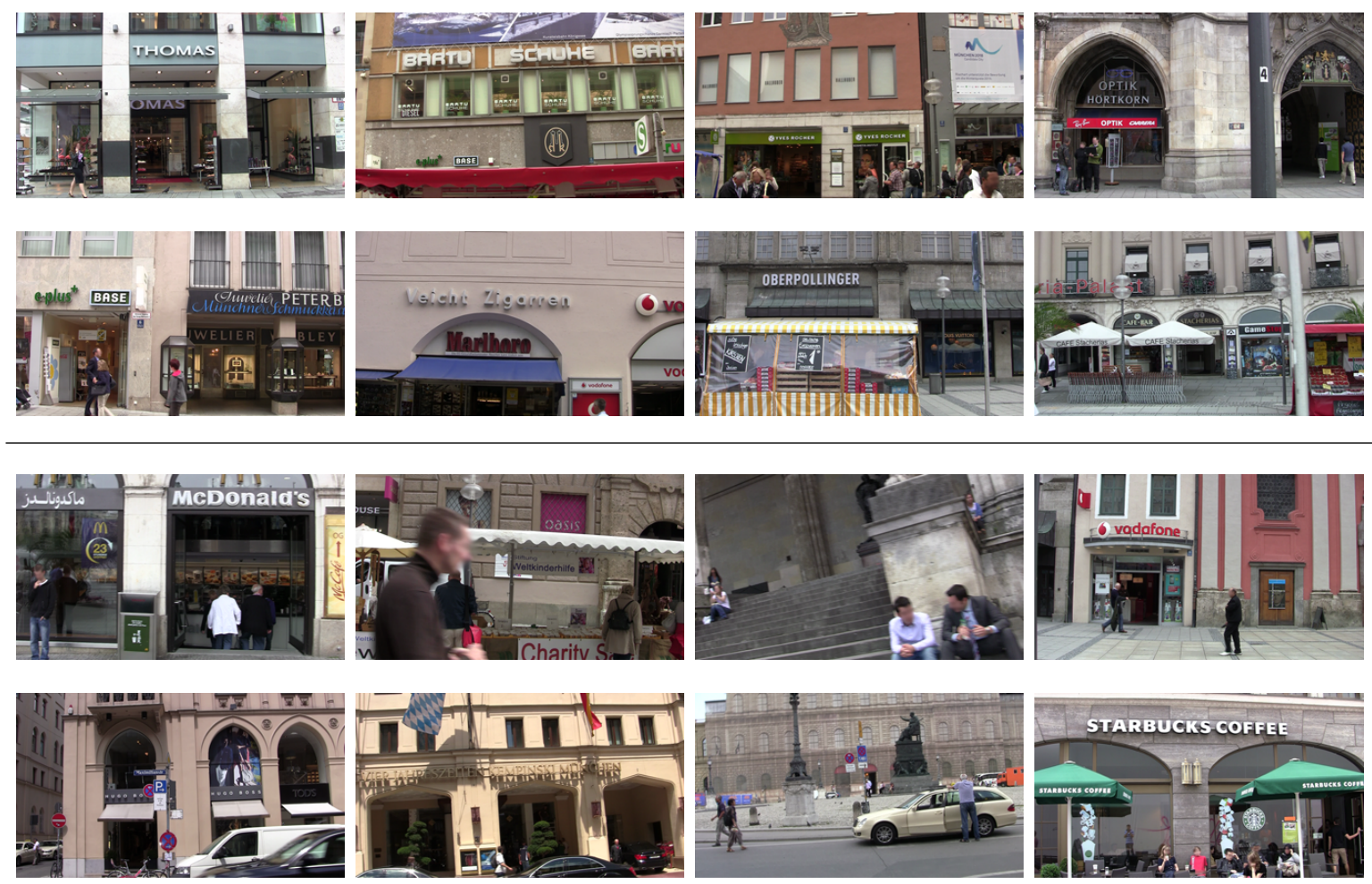

Figure 6.19: Random samples from the Munich City Center dataset. The two top rows show queries, the two bottom rows database images.

ments can be significantly reduced, which is of high interest in large scale product recognition applications. In mobile applications, the quantization of features into visual words can be performed on the device in real time due to the small vocabularies. Further, transmission delays are reduced as significantly less features have to be sent to a server.

Combining individual N-grams, as described by Equation 6.13, allows us to exploit their complimentary properties and results in increased performance as shown in Figure 6.17. In most cases, maximum performance is reached when combining all $\mathrm{N}$-grams with each other. We only considered N-grams up to an order of 4 as the gain in performance comes at the cost of increased memory requirements of the inverted file. Combining uni-, bi-, tri, and four-grams, the size of the inverted file is 4 times larger than with a single N-gram type, which is less than $3 \mathrm{MB}$ for 20000 images. At larger vocabulary sizes, the performance gain is less pronounced, which can be explained by the basic model to determine the probability of correct feature quantization.

In Figure 6.18, the mAP scores for the individual N-grams and their combinations are plotted against the radius defining the database images to be retrieved. Here, the vocabulary size is fixed to 400 visual words. Due to the comparably small vocabulary, which increases the chance that descriptors are assigned to the same visual word even for significant affine transformations, and the performance of the text detector, matching can be achieved across wide baselines.

To evaluate the robustness of the approach against highly dynamic and cluttered scenes, we 


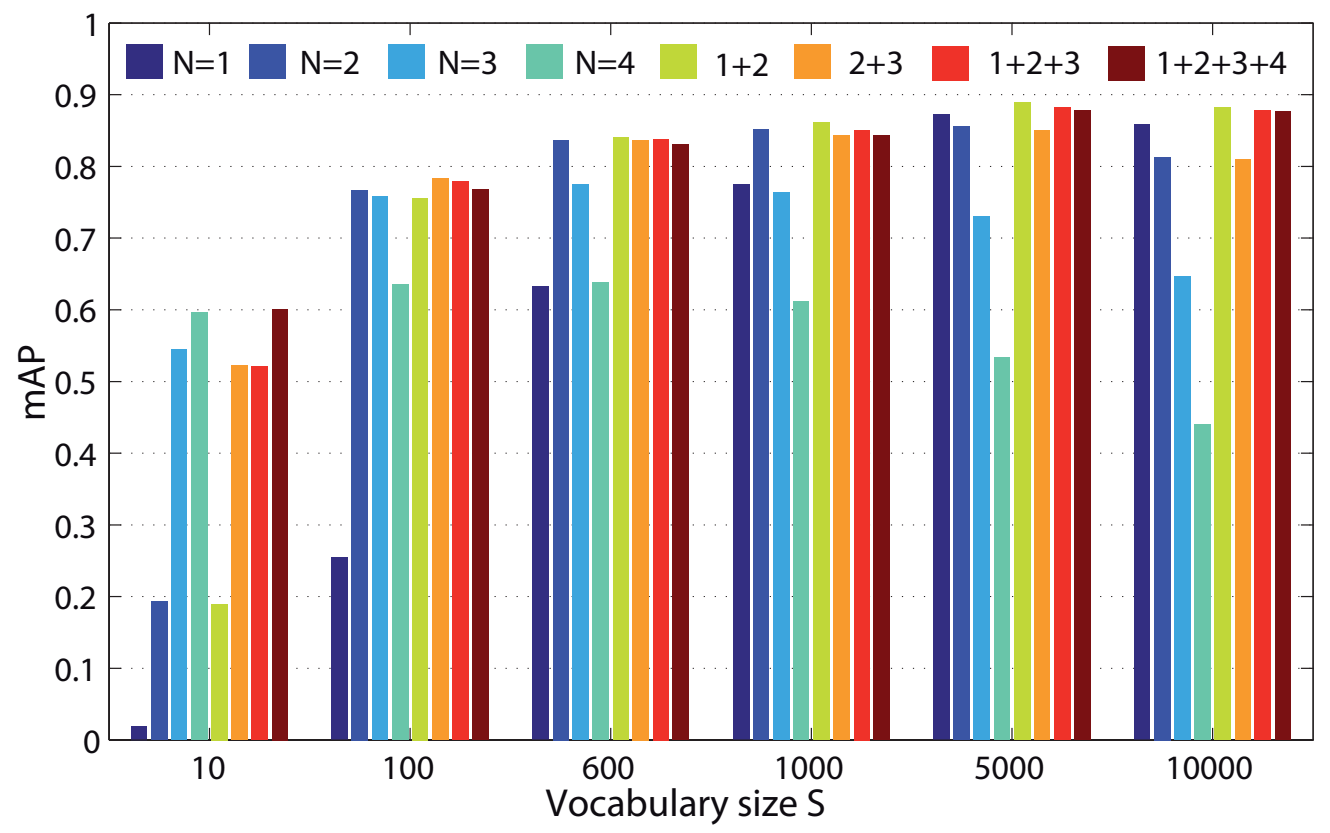

Figure 6.20: $m A P$ score of selected $N$-gram configurations at varying vocabulary size $S$ using the Munich dataset. (Reproduced from [SHH+11], (C) 2011 IEEE)

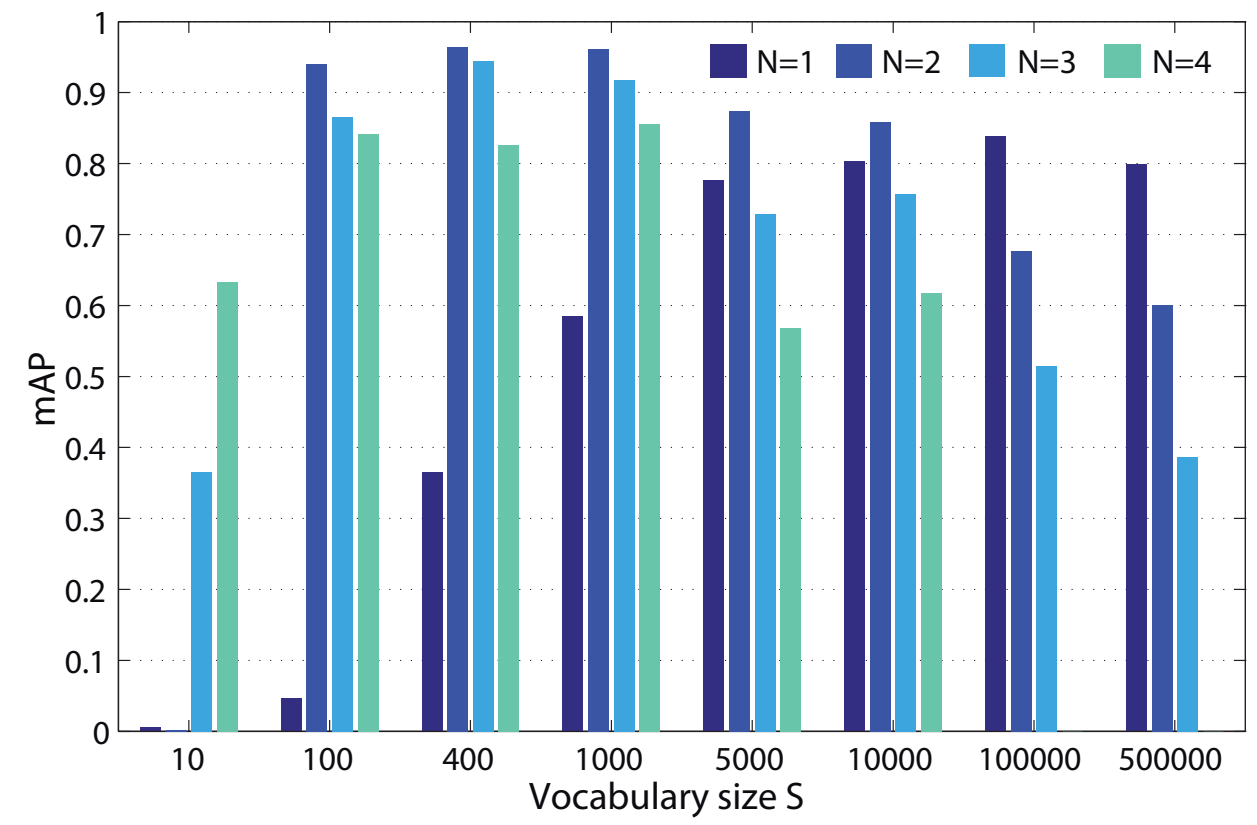

Figure 6.21: $m A P$ score of single $N$-grams at varying vocabulary size $S$ using the Amazon books dataset. (Reproduced from [SHH+11], (C) 2011 IEEE) 
extracted 7000 frames recorded with a camcorder along a $5 \mathrm{~km}$ long track on a shopping mile in downtown Munich for the second experiment. Query images are recorded at 109 locations and database images within a radius of 15 meters have to be retrieved. Exemplary database frames are shown in Figure 6.10 and 6.19 illustrating the amount of dynamic objects and clutter in this database. Plain BoF based approaches without higher level feature selection achieve an mAP of 0.73 at best. As mentioned in Section 6.2.1, a geometric verification using an affine model to rerank the top results does not increase the performance due to the clutter and the complex 3-dimensional scene. A significantly higher retrieval performance can be achieved when exploiting text related features only, as shown in Figure 6.20. Here, the mAP of single and combined N-grams is plotted against the vocabulary size $S$. Due to the frequent and large writings, we achieve an mAP of 0.89 by combining uni- and bi-grams
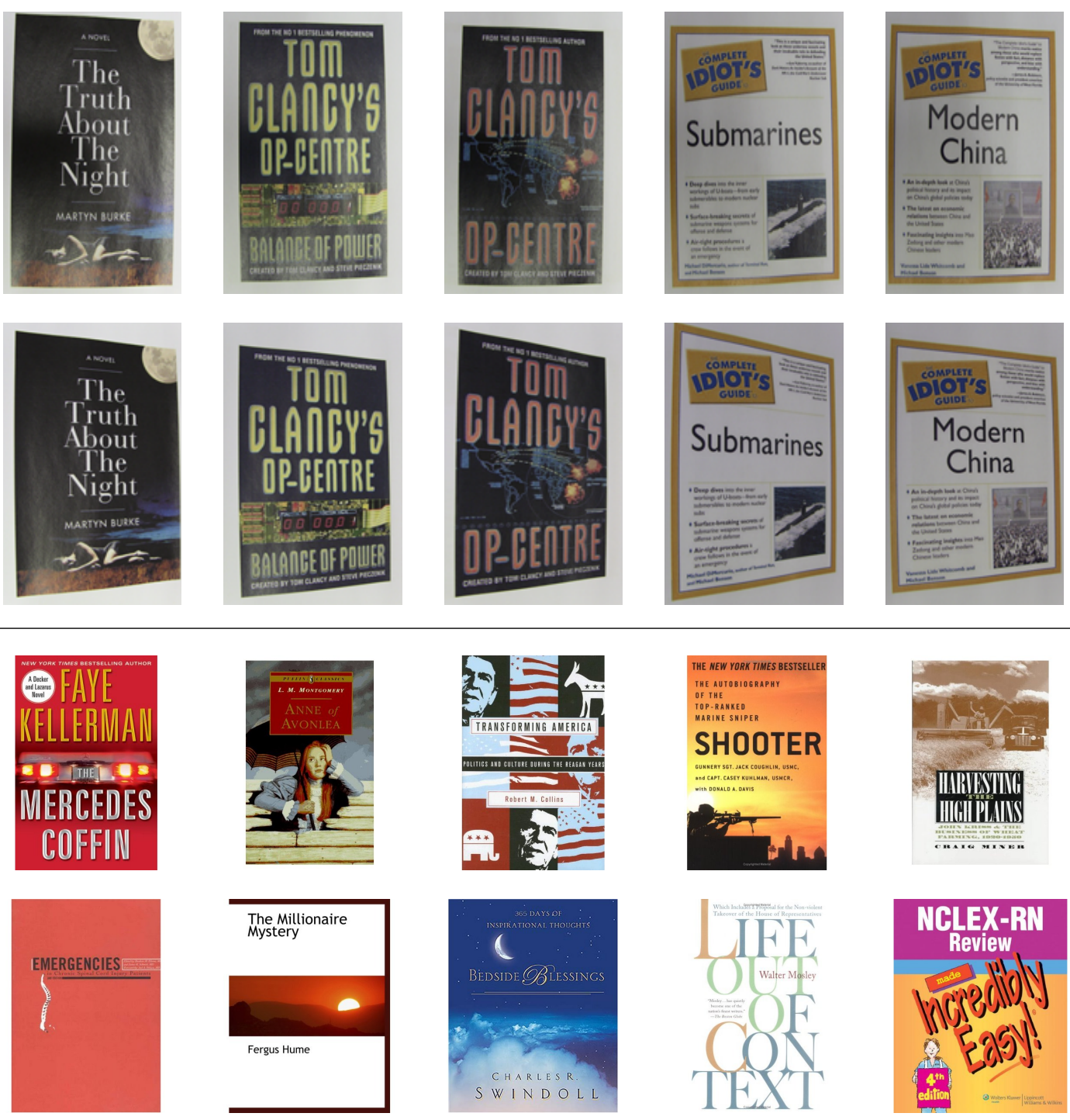

Figure 6.22: Random samples from the Amazon Book Covers dataset. The two top rows show query photographs, the two bottom rows database images. 
at a vocabulary size of 5000. In this dataset uni-grams can outperform bi-grams at larger vocabularies due to the high distinctiveness of store sign characters and the comparably small size of the dataset. It is worth noting that even at extremely small vocabularies with only 10 visual words, four-grams achieve a reasonable performance. The quantization complexity here is very low, which is of great benefit for mobile applications.

To evaluate the approach with respect to large scale datasets, we applied it to a product recognition task where the ID of a book has to be determined based on an image recording of its cover. The dataset comprises 203000 book covers provided by Amazon at a resolution of $500 \times 500$ pixels with noticeable compression artefacts. 60 query images are captured from different angles, varying between $0^{\circ}$ and $60^{\circ}$. Light reflections and slight motion blur complicate the retrieval as shown in Figure 6.22. Figure 6.21 shows the mAP scores of single N-grams for varying vocabulary sizes $S$. The performance of uni-grams peaks at an mAP of 0.83 using a vocabulary of 100000 visual words which is almost equivalent to plain BoF-based CBIR as mostly text related features can be found on book covers. Due to the size of the dataset and thus the variety of characters, uni-grams profit from large vocabularies. However, uni-grams are significantly outperformed by bi-grams achieving an impressive score of 0.97 at just 400 visual words.

Combined $\mathrm{N}$-grams did not provide a significant increase in performance as the results of the individual N-grams are very similar. Four-grams are not computed for vocabulary sizes of 100000 and 500000 as visual phrases are indexed with 8 byte in our current implementation. However, hashing techniques allow us to handle these large but extremely sparse feature sets.

\subsubsection{Door Sign Recognition}

As door signs are artificially created to enable humans to localize and orientate themselves in an unknown environment, recognizing them can be of high interest when considering an indoor navigation system. Identifying a sign allows us to obtain a precise estimate of the current location and orientation. As door sign typically follow a strict layout, a template can be used to generate virtual reference views of all doors signs existing in a building or even a whole company. With door signs being placed at known locations an indoor location recognition system can be installed at ease and without the need for mapping the environment.

In order to evaluate the retrieval performance of the visual phrase based approach to the task of indoor localization, an extensive door sign dataset has been created. As a query real images of actual door signs are recorded using a smart phone camera.

The TUMsigns dataset comprises 10100 computer generated door signs whereof 149 images are replicas of existing door signs at the main site of the university. The other 9951 images are generated with increasing door numbers and using random text. The resolution of the dataset is $2560 \times 2558$ pixels. The artificially generated door sign images have perfect edges and no blur is applied. The query dataset consists of 135 images recorded with a consumer smart phone at a resolution of 2560 x 1920 pixels. Again the MSER detector combined with the SURF descriptor is employed.

Three test cases are evaluated based on this dataset. Whereas in the first, no modification is applied, in the second case a fixed rotation of $30^{\circ}$ around the vertical axis is applied to evaluate the performance at slant viewing angles. In the third case, a random pan between 

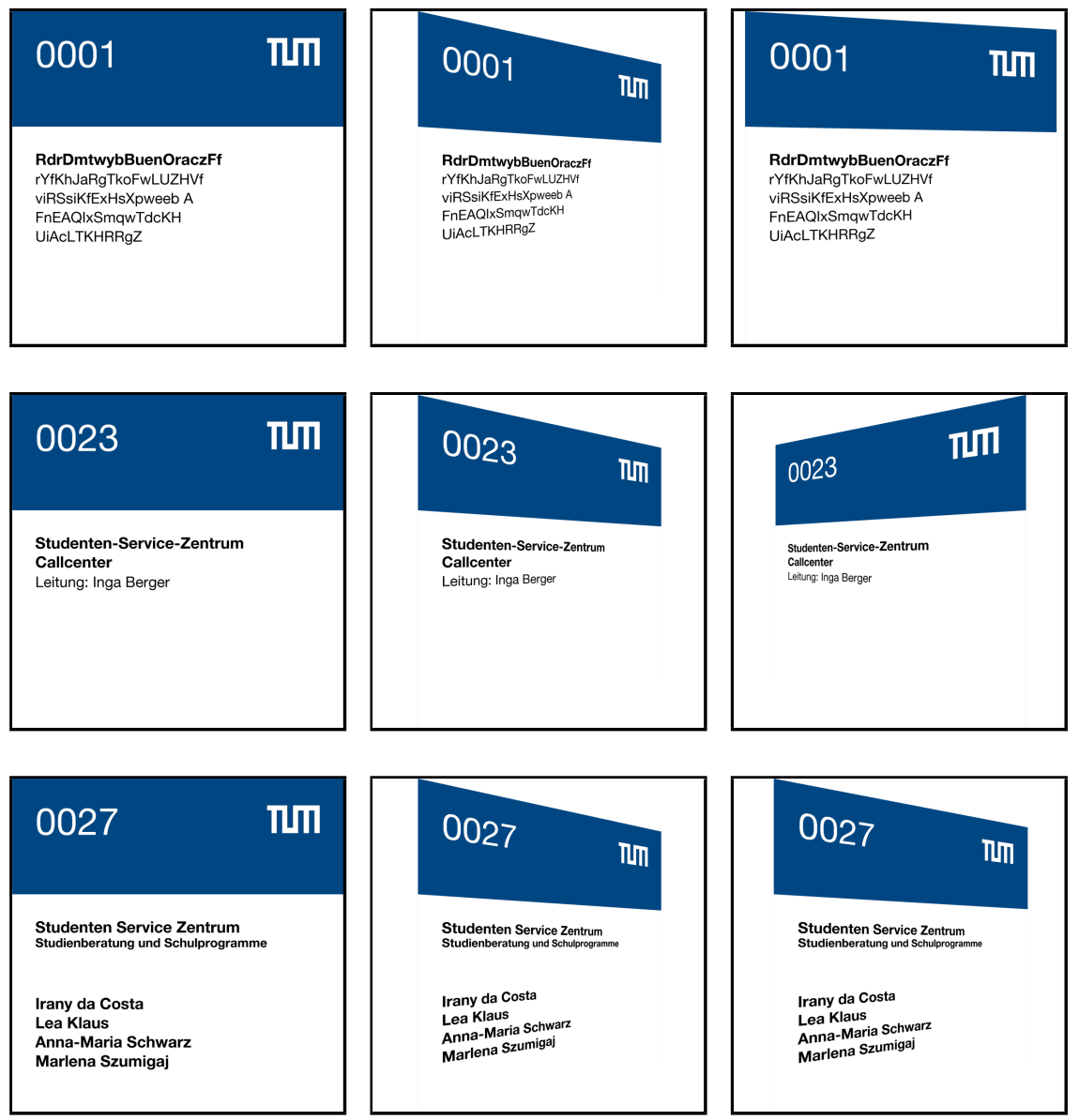

Figure 6.23: TUM Signs dataset examples (normal, fixed pan, random pan).

$-30^{\circ}$ and $+30^{\circ}$ around the vertical axis is applied.

Examples of the datasets care shown in Figure 6.23, where every column shows three examples from the same database. The door signs share the common shape with the logo on the upper-right side, an unique room number on the upper-left side and the names of the people in the room in the white area.

Figure 6.24 illustrates samples from the query dataset used for the evaluation. The query images are subject to different distortions, such as blur and varying light conditions.

As a baseline result, standard BoF based image retrieval is applied to the door sign recognition problem. To evaluate the performance, again the mAP score is employed and plotted against the vocabulary size $S$ in Figure 6.25. To achieve an mAP of 1 the corresponding artificial door sign has to be ranked first in the results for all queries. In this experiment the unmodified dataset without rotations has been used. As can be observed, the performance of the BoF based recognition peaks at a vocabulary size of 200. This corresponds with intuition as we have a very limited set of possible features that consists of letters, numbers, edges of the template and the TUM logo. Hence, the visual vocabulary very well captures the clusters of occurring image patches within the door signs. Performance, however, is very limited achieving a maximum $\mathrm{mAP}$ of 0.45 , which is clearly insufficient of the application to 

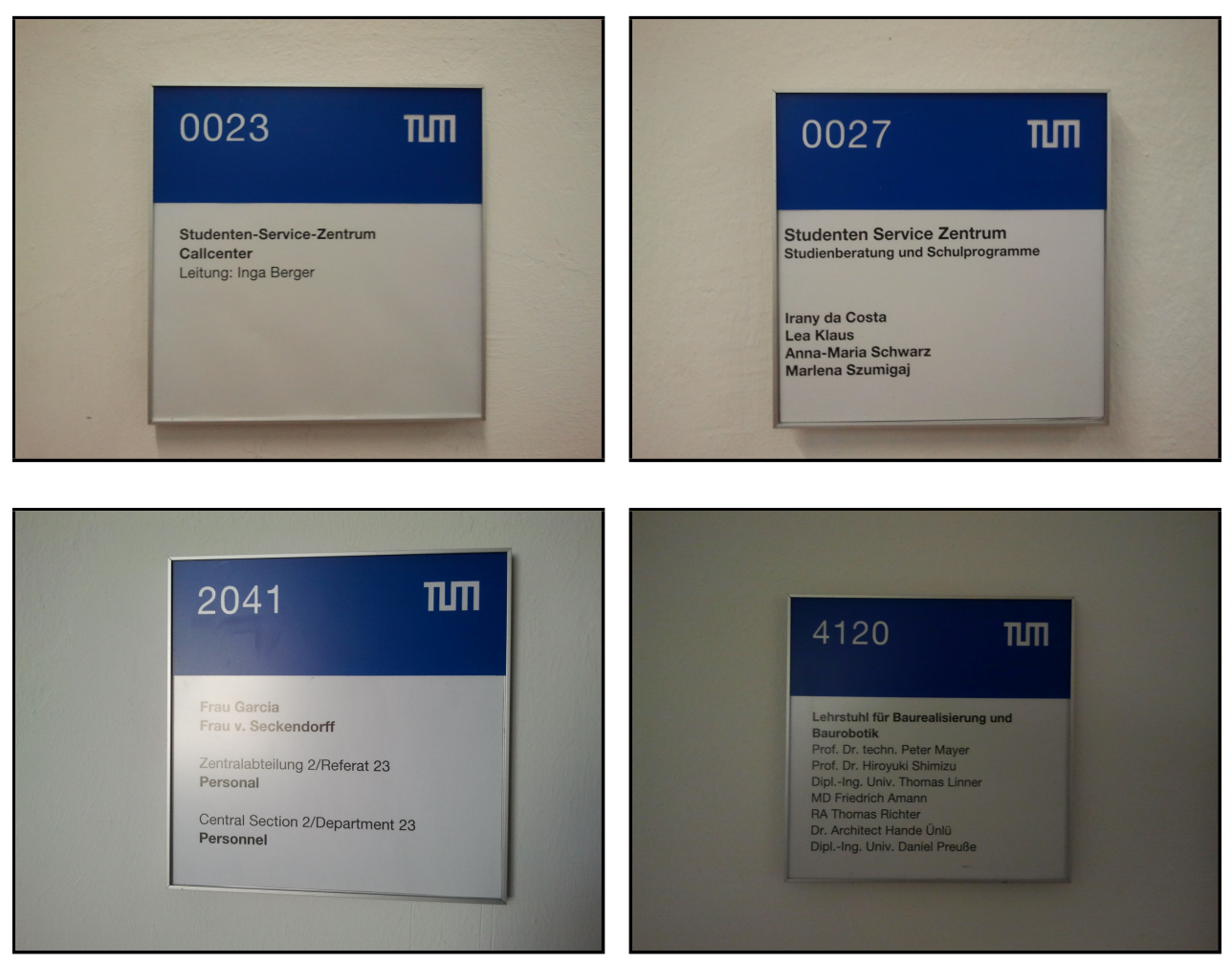

Figure 6.24: Examples of query images for the TUM Signs dataset.

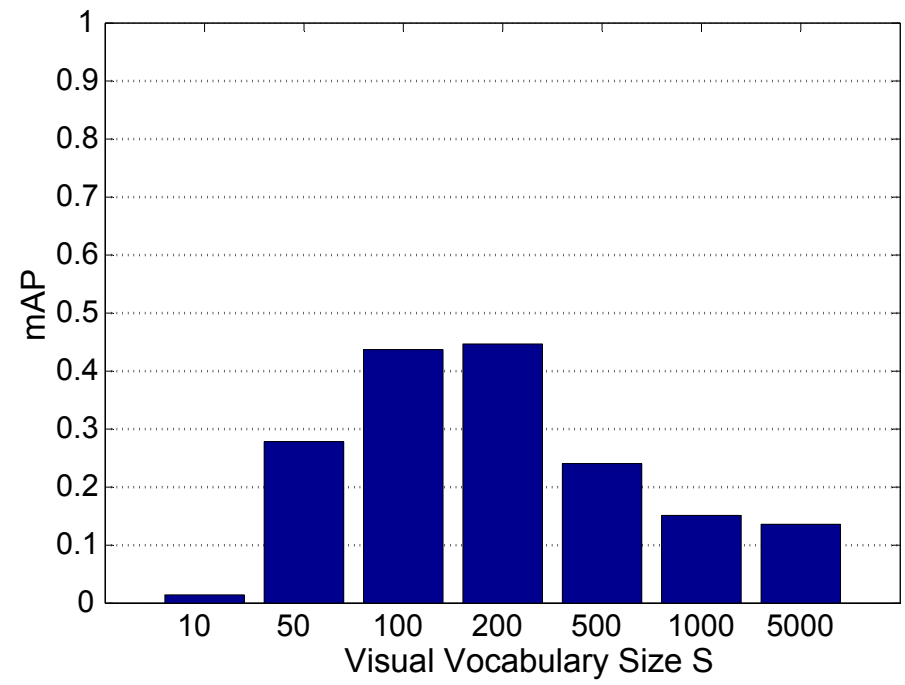

Figure 6.25: $m A P$ for the unmodified dataset using Bag-of-Feature retrieval over visual vocabulary size $S$. 


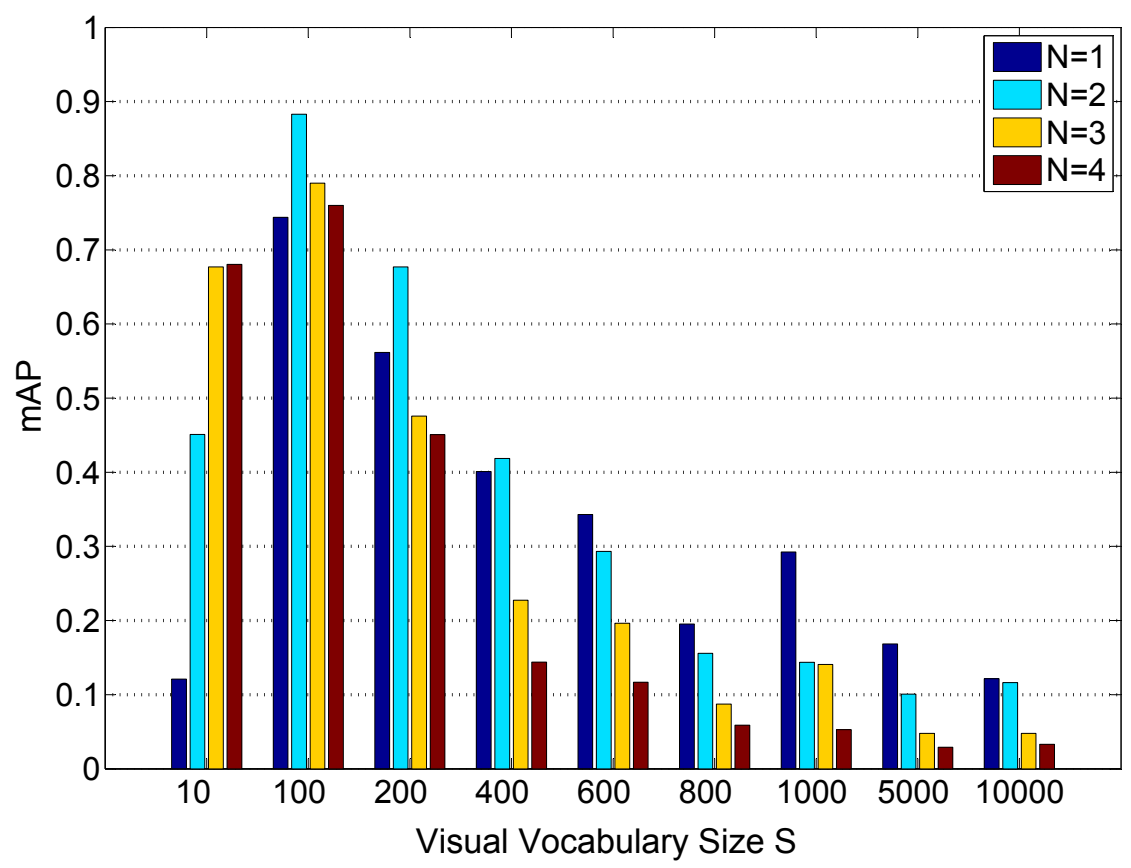

Figure 6.26: $m A P$ for normal dataset using text-related retrieval over visual vocabulary size $S$ and $N$-gram length $N$.

be perceived as useful.

To evaluate the performance of the visual phrase based approach, it is first applied to the unmodified dataset. The results in Figure 6.26 indicate a significant enhancement compared to the BoF based approach. As a first observation, it can be recognized that the performance of all N-gram configurations peak at a vocabulary size of 100 , which is lower than with the previously described BoF based approach. This is due to the fact that only text-related features are used to form N-grams and thus the set of image patches represented by visual words is now limited to characters. This is illustrated in Figure 6.27, where an artificially generated door sign is overlaid with the detected MSER features (shown as ellipses) and textrelated features respectively. Further, the edge-enhanced MSER, which is employed as part of the text detection engine described in Section 6.1.1, increases the repeatability of the detected features. This is achieved by combining MSER with the complimentary Canny edge detector, which significantly reduces the susceptibility against blur. These modifications result in the increased performance of N-grams with length one, which should otherwise perform similarly to standard BoF as mostly text is visible within the signs.

The maximum performance is achieved with $\mathrm{N}$-grams of length two, resulting in an mAP of 0.883. These results confirm that including geometric dependencies is vitally important in this context. This performance can fairly be assumed to be sufficient for a door sign recognition based localization system to be perceived as useful.

These results, however, are achieved when the viewing angle between the query and reference image is only slightly tilted (up to a maximum of $20^{\circ}$ ). To evaluate the stability with respect to strong perspective changes, the modified dataset with a fixed pan of $30^{\circ}$ is used. As the query images stay the same, this experiment determines the impact of severe viewpoint 


\begin{tabular}{|l|}
\hline 1119 \\
Studenten Service Zentrum \\
- Organisation und IT - \\
Angelika Müller \\
Irene Proniewick \\
\hline
\end{tabular}

(a)

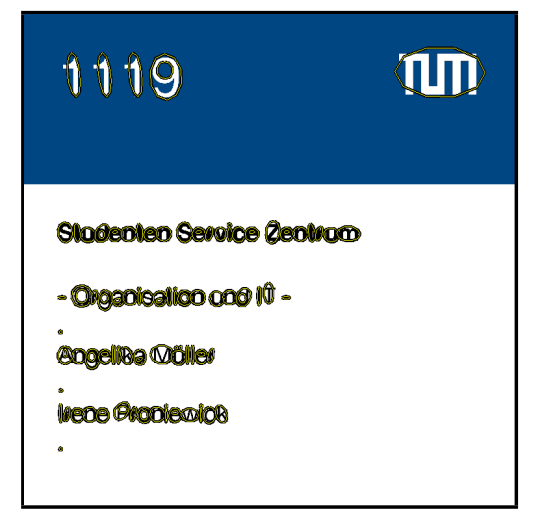

(b)

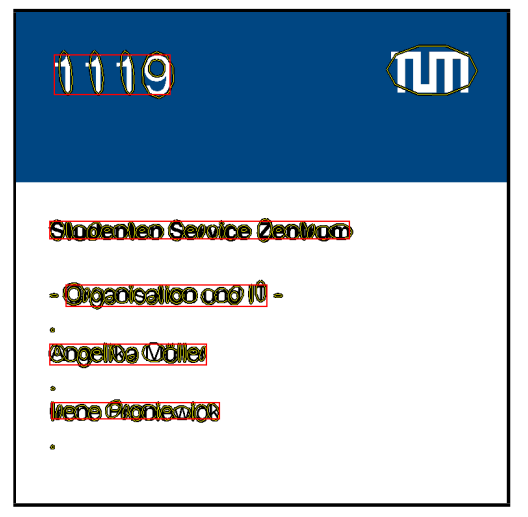

(c)

Figure 6.27: Comparison of MSER and text-related features. An artificially generated door sign is overlaid with MSER features in (b) and with text-related features in (c) respectively.

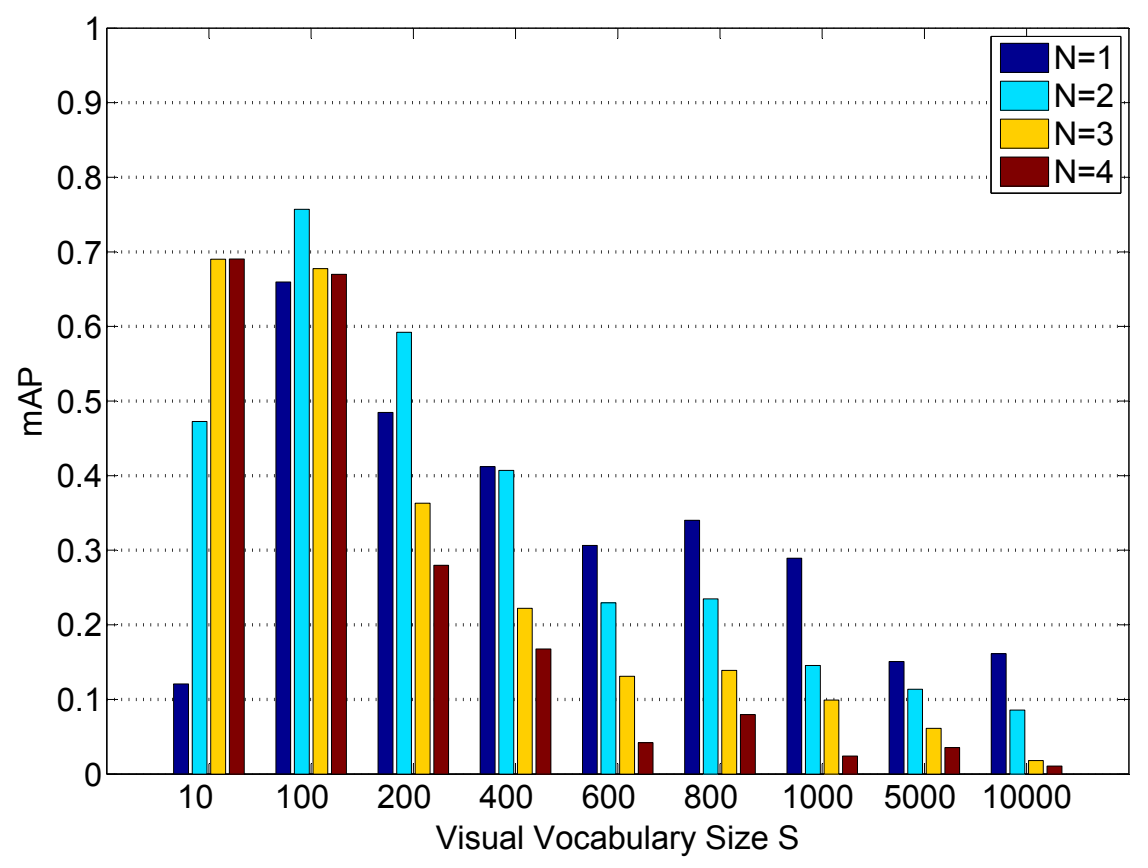

Figure 6.28: $m A P$ for fixed panned dataset using text-related retrieval over visual vocabulary size $S$ and $N$-gram length $N$.

changes and thus allows us to determine the performance in the case the user is viewing the door sign from the side.

When comparing the results shown in Figure 6.28 to those in Figure 6.26, only a slight degradation in mAP can be observed. This is mainly due to the affine invaiance of the MSER 


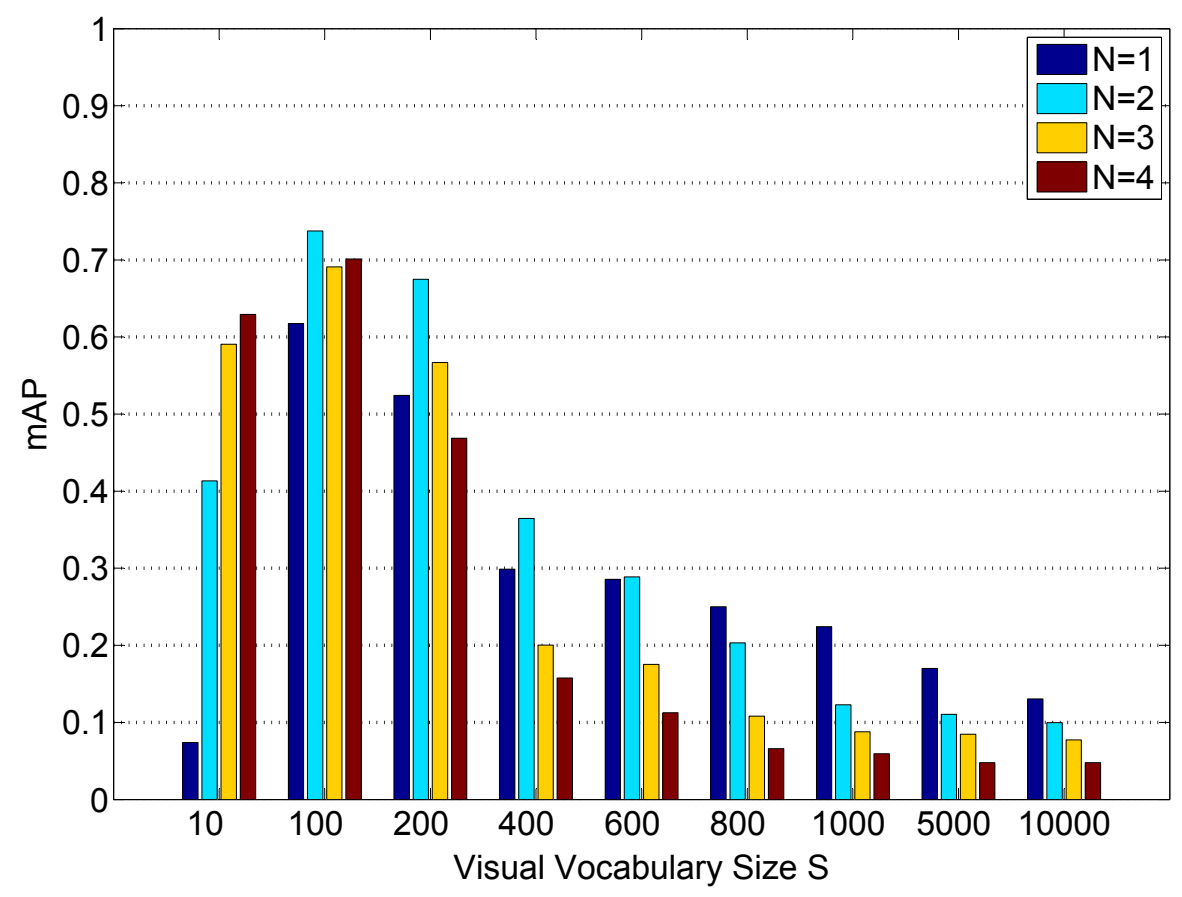

Figure 6.29: $m A P$ for randomly panned dataset using text-related retrieval over visual vocabulary size $S$ and $N$-gram length $N$.

detector as well as the performance of the text detection engine, which can handle also very slant views. The last modification to the door sign dataset with random pans between $+30^{\circ}$ and $-30^{\circ}$ shows comparable results in Figure 6.29 .

\subsection{Summary}

In this section, a novel approach to exploit text-related features for image retrieval applications is presented. In contrast to optical character recognition (OCR) based retrieval engines, in this visual phrase based approach writings only need to be detected and localized rather than recognized. This allows us to be significantly more robust to low resolution and blur.

The detection and localization of text is performed using the so-called edge-enhanced MSER (EMSER) approach, which is introduced in Section 6.1.1. This text detection engine achieves state-of-the-art performance, while requiring very low computational complexity, which makes it particularly suited for mobile applications. This is achieved by generating basic letter candidates using the MSER interest region detector (requiring 30ms for $640 \mathrm{x} 480$ at $3 \mathrm{GHz}$ ), which is already part of state-of-the-art image retrieval approaches.

Detected and localized characters are described using robust feature descriptors like SURF [BETG08 and quantized into visual words using an approximate k-means algorithm. In contrast to an alphabet of about 30 characters in OCR-based approaches, this visual phrase based approach differentiates between approximately 600 visual words by considering the 
appearance of the individual character font. Characters within a writing are combined to Ngrams (i.e. substrings of length $\mathrm{N}$ ) to exploit the geometric relationships while being robust against detection and quantization errors. The approximate string matching is efficiently performed using inverted files. Estimating the average information obtained from specific $\mathrm{N}$-grams allows us to choose the optimal length $\mathrm{N}$ as well as to combine different $\mathrm{N}$-gram types in order to exploit their complimentary properties, hence increasing the overall retrieval performance.

Compared to state-of-the-art BoF-based retrieval systems, the number of required features as well as the size of the visual vocabulary is significantly reduced. Thus, the overall memory requirements of the database are reduced by two orders of magnitude. With the increased distinctiveness of $\mathrm{N}$-grams, a significantly increased retrieval performance, especially for large scale databases, is achieved at the same time. Due to the high level feature selection based on text, the amount of features on clutter and dynamic objects is reduced as shown in Figure6.10.

In Section 6.3.1, the application of this visual phrase based retrieval engine to the indoor location recognition task is demonstrated. Door signs at the campus of the Technical University of Munich have to be retrieved based on the recordings of a mobile device. The use of visual phrases based on text-related features clearly outperforms BoF-based retrieval approaches. This experiment is also a good example for an indoor localization system that does not require a tedious mapping of the environments as the template of the door signs as well as the associated location as usually known.

Further, the visual phrase based approach can be efficiently combined with traditional BoFbased retrieval systems, to cope with databases where little or no text is available as the same features (MSER) and descriptors (SURF) can be shared.

The basic idea of bundling features to visual phrases is not limited to text-related features but can be applied with alternative methods that ensure planar structures. A possible approach would be to detect planar areas in the image via collinear features, which can be found on buildings and in hallways as argued by Rajashekhar et al. in [CN07]. In indoor environments, where spatially close features often lie on a plane, it could be sufficient to simply combine features within a given radius to N-grams. Preliminary experiments, however, show that it requires very stable and repeatable features for the bundling to outperform classic BoF. Whereas text-related features go through a sophisticated detection engine and are shown to be stable with respect to perspective transformations (see Section 6.1.1), regular features selected by interest point or region detectors provide a comparably low repeatability, which is insufficient when combined in a feature bundle. 


\section{Virtual Views for Meter-Accurate Localization and Orientation Assignment}

प

In the system architectures presented in the previous chapters the location of the mobile device is estimated by determining the visually most similar geo-tagged database image, e.g., a $360^{\circ}$ panorama. These reference images are recorded at various locations during the mapping of the environment, while corresponding map coordinates are stored. In the case of indoor environments, this is achieved by a mapping trolley, which is described in the following Section 7.1. Although automated to a large degree, mapping buildings on a large scale is a time-consuming and tedious endeavor, which has to be minimized in order to allow for a widespread adoption of visual localization. Hence, in practice, reference images are captured along a single trajectory only. This results in two major limitations.

First, the actual location of the mobile device can only be mapped to the closest location on the mapping trajectory, drastically limiting the resolution of position estimates as returned by the image retrieval process. Further, without recording reference images in multiple directions, no orientation estimation can be performed, which is essential for location based services to be perceived as useful.

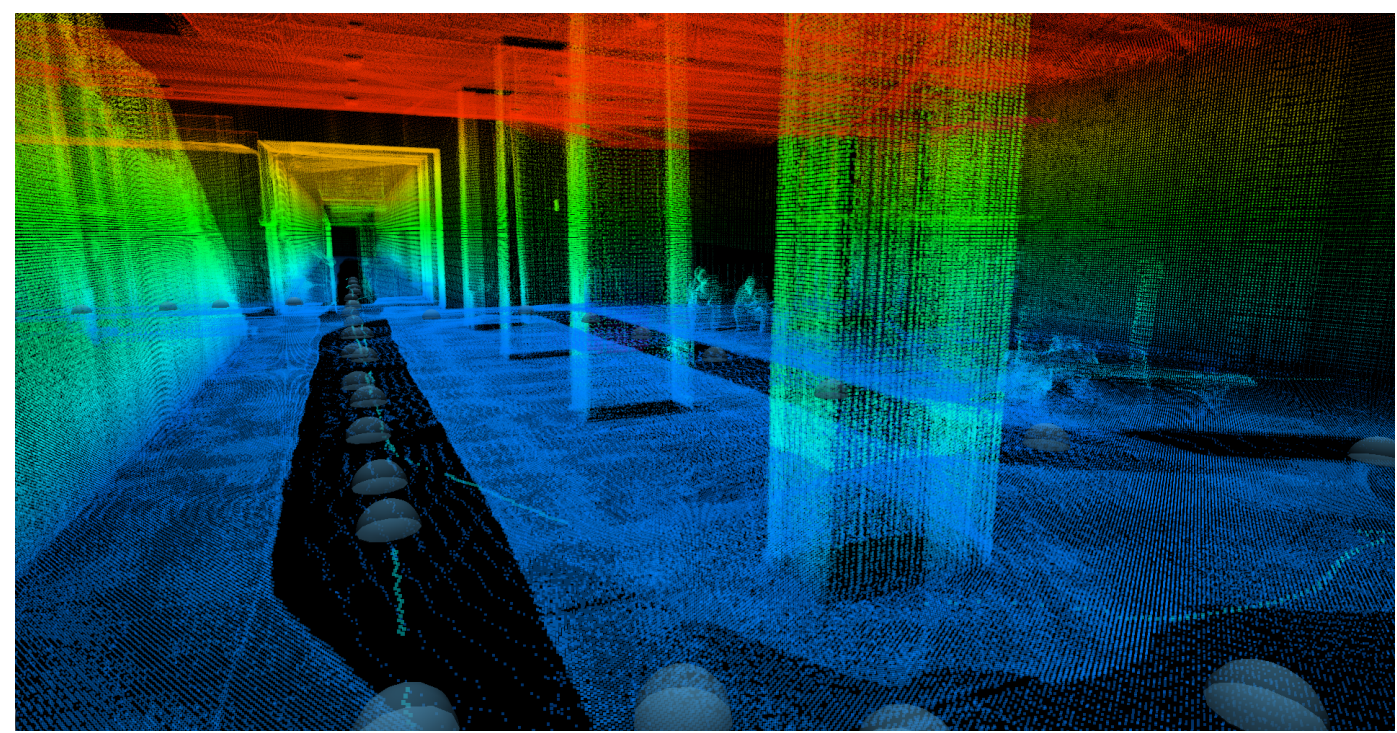

Figure 7.1: Point cloud acquired by registering scans from the vertically mounted laser scanner. By casting rays from camera positions into the point cloud, world coordinates of local image features are determined. (Reproduced from [HSH+12b], (c) 2012 IEEE)

Parts of this chapter have been published in $\mathrm{HSH}^{+} 12 \mathrm{a}$, and $\mathrm{HSH}^{+} 12 \mathrm{~b}$. 
Second, the limited affine and perspective invariance of feature descriptors is a severe problem, as a location can be recognized only if a reference image with a pose similar enough to the query image exists. There has been extensive work on improving the robustness of feature descriptors under perspective distortion. However, usually robustness is gained at the expense of distinctiveness, hence, these approaches tend to increase recall only, but not precision.

In Section 7.2, the so called virtual view based localization is introduced, which generates virtual reference images at all possible locations and orientations within a mapped environment. This is achieved by exploiting planar structures detected in the 3D point cloud recorded by the mapping trolley introduced in Section 7.1. Virtual views enable us to determine camera poses far off the mapper trajectory solely based on image retrieval results.

With the increased number of reference images, the visual similarity between the query image and the spatially closest database image is increased. Hence, it can be assumed that a high invariance of feature descriptors with respect to affine transformations is no longer required but rather reduces the distinctiveness. Recently, the so called Binary Robust Elementary Features (BRIEF) [CLSF10] have been proposed, which are reported to be up to 40-fold faster than SURF KCS11] at the cost of limited invariance and repeatability. In Section 7.3. an adapted quantization structure is proposed to integrate binary features into the visual localization system described in the preceding chapters. Section 7.4 evaluates the virtual view based localization system using fast binary features.

\subsection{Mapping Platform}

The basis for vision based indoor location and orientation recognition is the mapping of the environment we want to navigate in. For this purpose, images have to be recorded and their corresponding pose needs to be determined. As will be described in Section 7.2 the generation of virtual views requires measuring the 3D geometry of the environment as well. Figure 7.2 shows a picture of the built mapping trolley including close-ups of the major sensors. To determine the pose of the trolley in previously unseen environments, a simultaneous localization and mapping (SLAM) has to be performed. This could be done using vision only via Structure-from-Motion (SfM) approaches (see Sections 2.1.1 and 2.1.2). As in buildings, areas (e.g., hallways) with very few visual features can be found, visual SLAM would result in an ill-conditioned problem.

In contrast to the localization of the user, however, we do not face the constraints of being limited to sensors existing in today's mobile devices. Thus, it is reasonable to make use of a laser range finder that scans the environment along the horizontal axis with a $190^{\circ}$ field-ofview. Mostly flat surfaces are assumed indoors, which allows us to use a trolley. This not only reduces the pose estimation problem to translations in $x, y$, and a rotation along the $z$ plane (yaw) but also allows for the use of wheel odometry. Established SLAM algorithms from the robotics community, which fuse laser scans with wheel odometry, like GMapping can be used to obtain precise pose estimates [GSB07. In GMapping a Rao- Blackwellized particle filter is employed to learn occupancy grid maps, shown in Figure 7.4, of the environment. White areas indicate free space that was traversed by a laser beam, whereas black areas indicate a wall at which a laser beam was reflected. In Figure 7.4a, the occupancy grid map of a long mapping run $(1169 \mathrm{~m})$ is overlaid with an aerial image of the main campus of the Technische Universität 

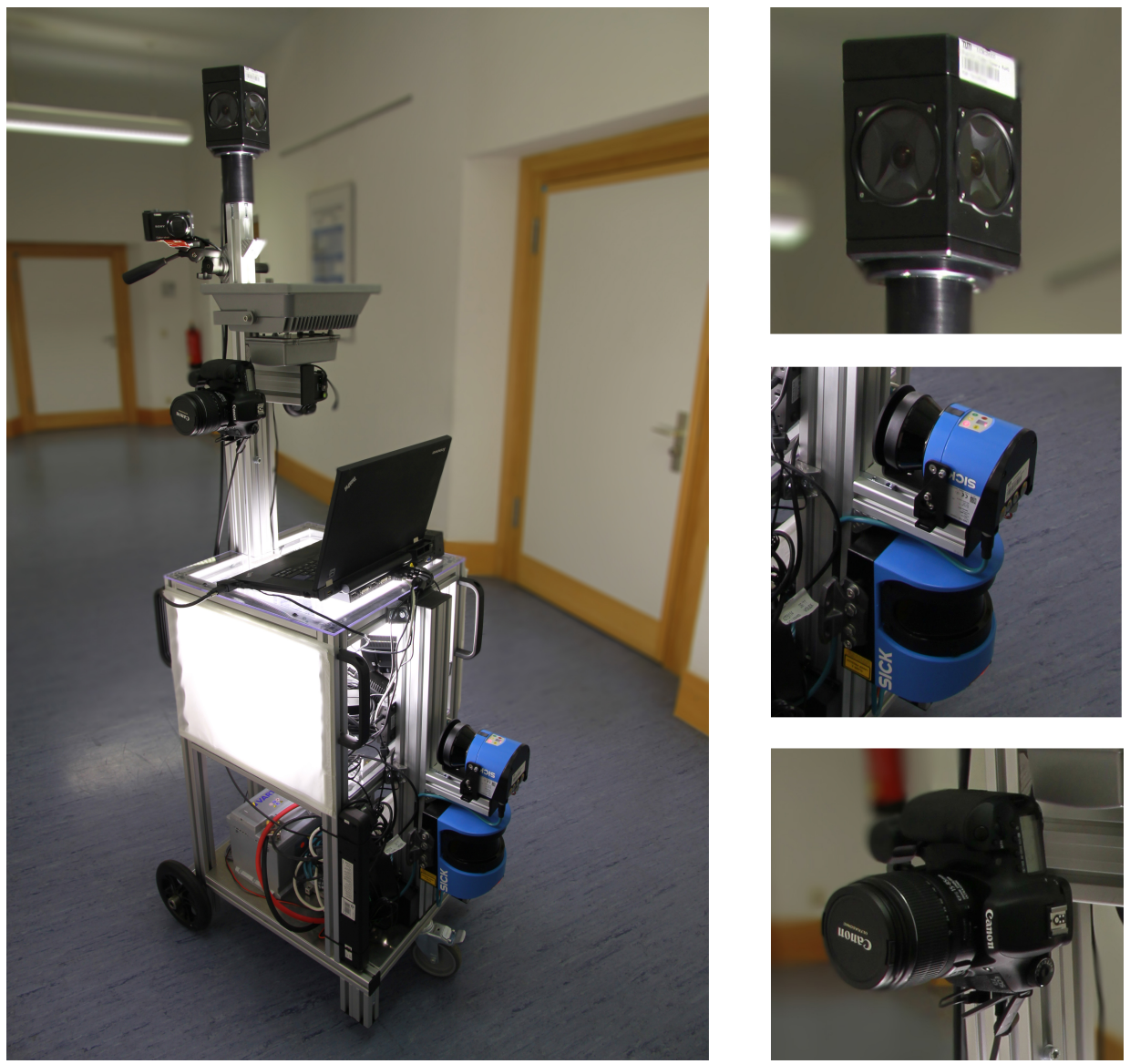

Figure 7.2: The mapping trolley used for dataset acquisition. (Reproduced from [HSH+12a], (c) 2012 IEEE)

München. Although no loop closure was performed, the grid map almost perfectly matches the true layout of the hallways of the buildings. This is mainly due to the well calibrated sensors as well as the wide range $(80 \mathrm{~m})$ of the used horizontal laser scanner.

To measure the 3D geometry of the environment, a second vertically mounted laser range finder with a $270^{\circ}$ field-of-view incrementally constructs a model as the trolley is pushed through the hallways. Due to the use of a wheel odometry, the relative pose changes between successive measurements of the second laser range finder is very precise and smooth such that the pose estimation errors are below the noise of the laser scans $(2 \mathrm{~cm})$. Hence, impressive $3 \mathrm{D}$ point clouds, as the one shown in Figure 7.1 can be generated for large environments.

To obtain a complete visual recording of the environment a panoramic camera (PTgray Ladybug3) takes an overall of 6 overlapping wide angle images, which can be used to stitch a $360^{\circ}$ spherical panorama. Two Canon 7D DSLR cameras are recording high resolution images to exploit text-related features as described in Section 6 and to generate virtual views. Further, two soft LED lights to the side and one LED uplight pointed at the ceiling are indirectly lighting up the vicinity to reduce motion blur and image noise. All cameras are triggered synchronously approximately every $1.2 \mathrm{~m}$ and the corresponding pose is stored. Exemplary images recorded at a single location are shown in Figure 7.3 . 
While the LEDs have a high efficiency, they still consume the most power. Thus a $100 \mathrm{Ah}$ battery allows us to operate the trolley for approximately 3-4 hours. As slow walking speed is required to avoid motion blur in the images (typically a shutter time of $1 / 80 \mathrm{~s}$ is applied), about $1.5 \mathrm{~km}$ of hallways can be easily mapped with one battery.

Table 7.1 shows the datasets that have been recorded in November and December 2011. In total, more than 40000 high resolution images have been captured over a distance of $4.5 \mathrm{~km}$. The floor plans included in these datasets are shown in Figure 7.4. Further, 42 query video snippets with a resolution of 1920x1080 pixels have been recorded four months later with a Canon Legria HFS 21 camcorder. Each video snippet is a pan of about $60^{\circ}$ around a fixed location. Six frames are extracted from the individual snippets, which form the query for the following experiments. The ground truth locations are shown as red crosses in Figure 7.6 and query frames are illustrated in Figure 7.5. Only the first of the six frames per snippet is considered in these figures. The datasets are publicly available at the NAVVIS project website (http://www.navvis.de) and can be interactively browsed with the TUMindoor Viewer online.

\subsubsection{Outlook to Upcoming Mapping Platform}

The presented mapping trolley allows us to efficiently map large scale indoor environment both visually and spatially. However, there are still shortcomings which should be improved when designing the next mapping platform. Currently, the trolley is limited to flat surfaces and cannot be lifted on staircases due to its heavy weight. The $20 \mathrm{~kg}$ battery is currently required to power the LED lights used to reduce the shutter times of the panoramic camera (Ladybug3). While the camera allows for $10 \mathrm{fps}$, the resolution is insufficient to read text on walls and provides only a low photosensitivity. Currently, the camera system is augmented by

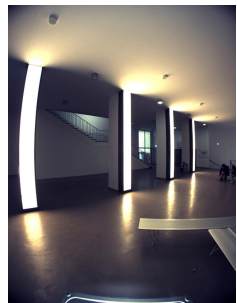

(a) Ladybug 0

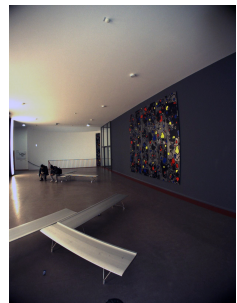

(b) Ladybug 1

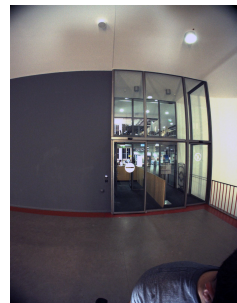

(c) Ladybug 2

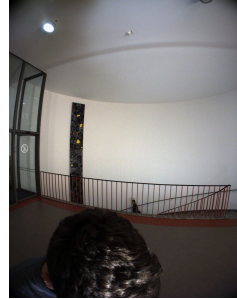

(d) Ladybug 3

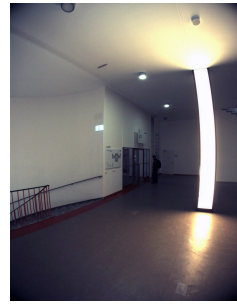

(e) Ladybug 4

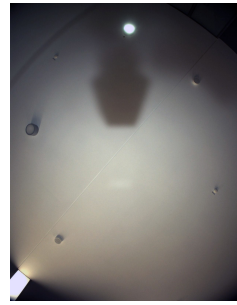

(f) Ladybug 5

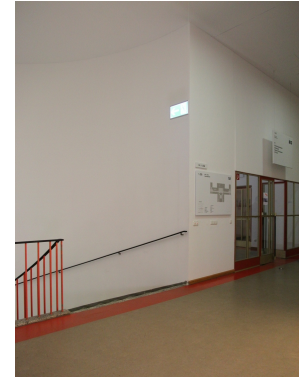

(g) Left DSLR

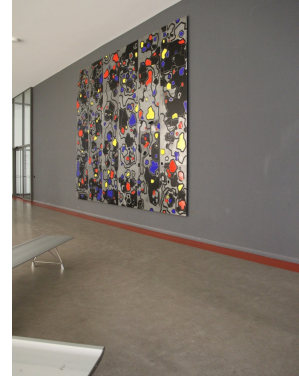

(h) Right DSLR

Figure 7.3: The eight images captured by panoramic camera and the two DSLRs every 1.2 $\mathrm{m}$. (Reproduced from [HSH ${ }^{+}$12a], (C) 2012 IEEE) 


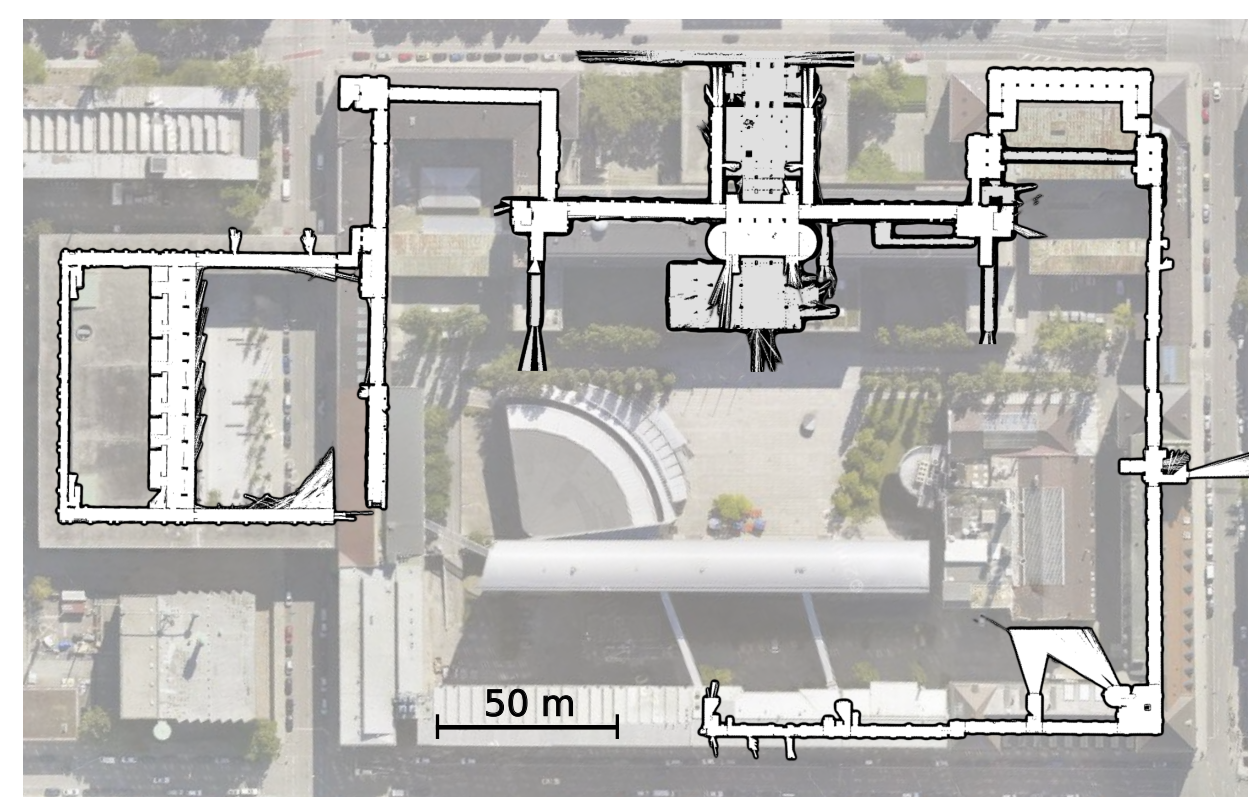

(a) TUM campus with plans of ground (gray) and 1st floor (white) ${ }^{1}$
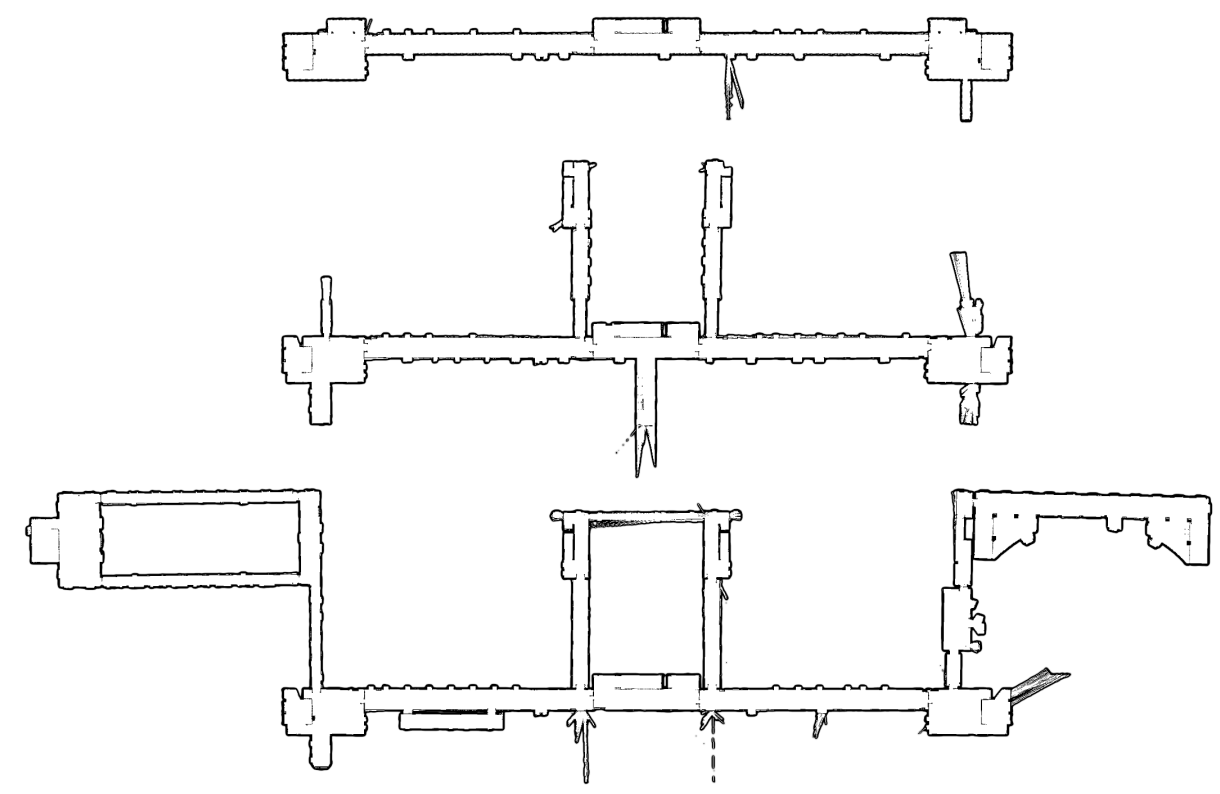

(b) 2nd, 3rd and 4th floor (from bottom to top)

Figure 7.4: Maps built with data from horizontally mounted laser scanner. The total distance traveled during acquisition is $4.5 \mathrm{~km}$; the length of mapped corridors is about $2.3 \mathrm{~km}$. (Reproduced from [HSH+12a], (C) 2012 IEEE) 

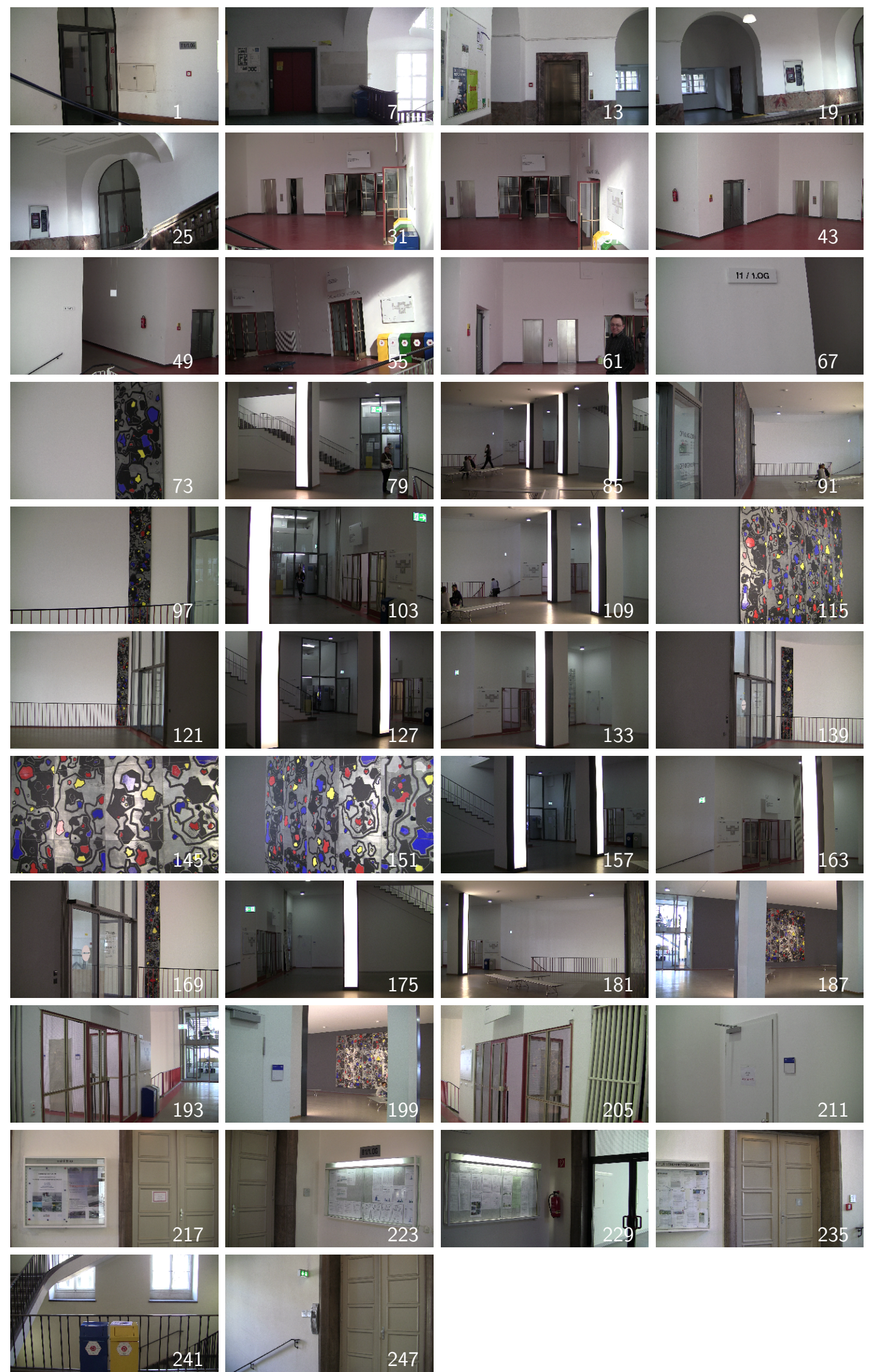

Figure 7.5: Sample images and frame numbers from query video snippets. 


\begin{tabular}{l|l|r|r|r} 
Run & Location & Images & Length & Points \\
\hline \hline $11-11-28$ & 1st floor & 12584 & $1169 \mathrm{~m}$ & $59.7 \mathrm{M}$ \\
\hline $11-12-13$ & 1st floor N1 & 7512 & $838 \mathrm{~m}$ & $34.0 \mathrm{M}$ \\
\hline $11-12-17 \mathrm{a}$ & 4th floor & 2312 & $246 \mathrm{~m}$ & $10.1 \mathrm{M}$ \\
\hline $11-12-17 \mathrm{~b}$ & 3rd floor & 3376 & $396 \mathrm{~m}$ & $14.7 \mathrm{M}$ \\
\hline $11-12-17 \mathrm{c}$ & Ground (I) & 5144 & $569 \mathrm{~m}$ & $23.3 \mathrm{M}$ \\
\hline $11-12-18 \mathrm{a}$ & Ground (II) & 5008 & $523 \mathrm{~m}$ & $16.9 \mathrm{M}$ \\
\hline $11-12-18 \mathrm{~b}$ & 2nd floor & 5952 & $781 \mathrm{~m}$ & $25.7 \mathrm{M}$ \\
\hline Total & & 41,888 & $4522 \mathrm{~m}$ & $184.5 \mathrm{M}$ \\
\hline
\end{tabular}

Table 7.1: Overview of mapping runs. Two 18 Megapixel digital single-lens reflex images and six 2 Megapixel Ladybug images are captured at each location and are tagged with the corresponding 6-DOF pose.

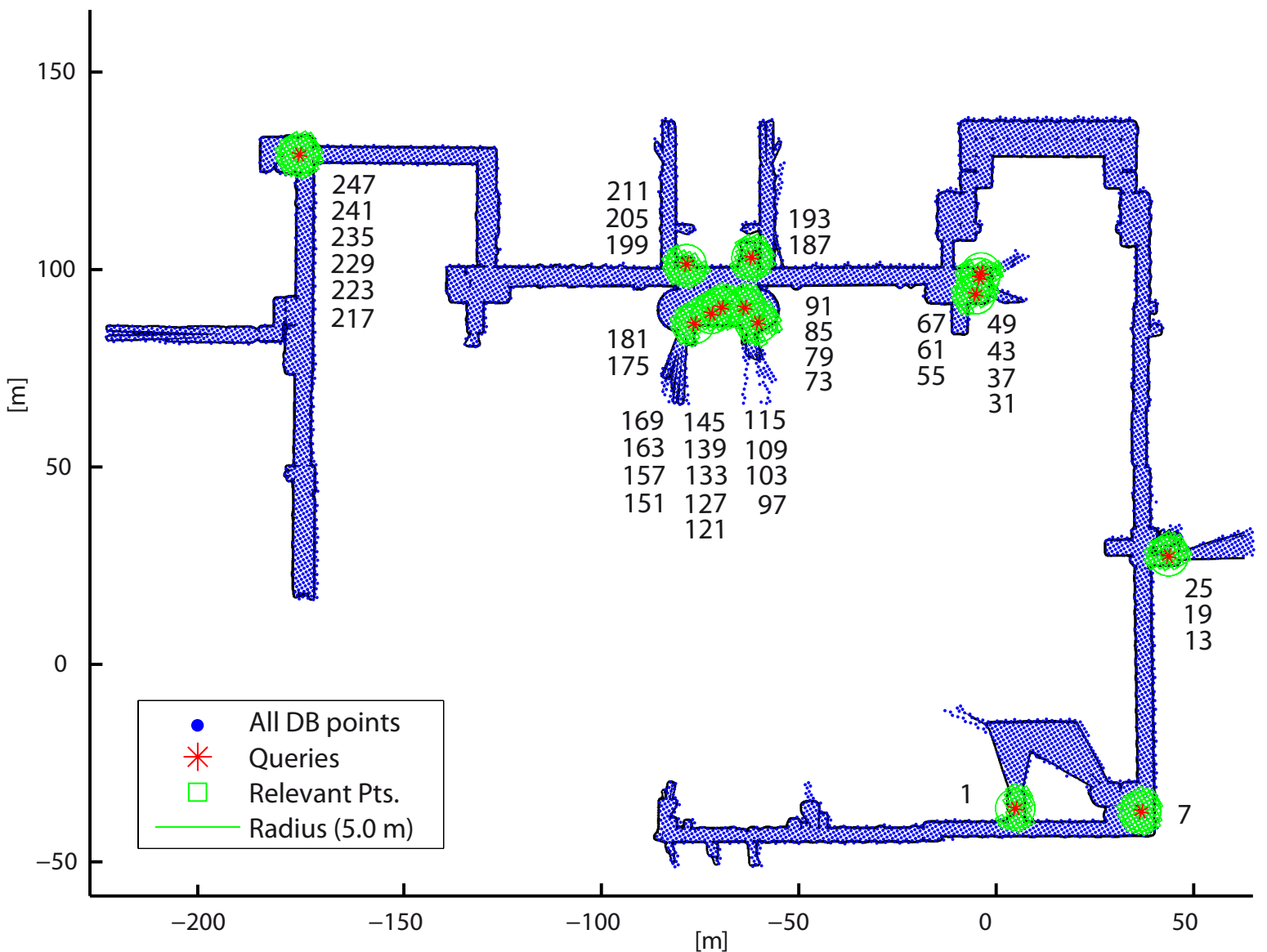

Figure 7.6: Locations of the generated virtual views (blue dots), the query frames (red crosses), as well as the radius around the query location defining the relevant virtual view points. A radius of $5 \mathrm{~m}$ is applied. 

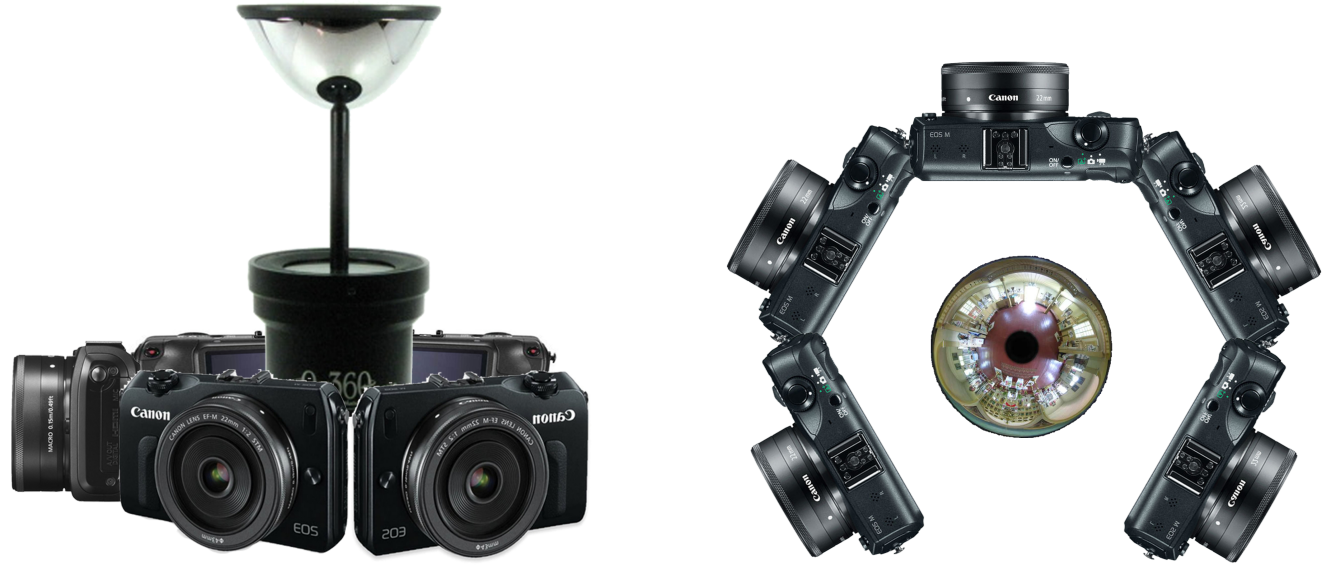

Figure 7.7: Illustration of the camera setup for the upcoming mapping platform.

two additional DSLR cameras pointed to the side. The vertically mounted laser scanner used to incrementally build a 3D point cloud of the environment is a very efficient solution to avoid heavy and expensive Velodyne $360^{\circ}$ laser range finders. However, when moving the trolley down a hallway, obstacles like columns occlude the area behind. To avoid the generation of incorrect 3D models, the trolley is usually turned from time to time to cast laser beams from different directions and to resolve these occlusions.

The group of Avideh Zakhor at the Video and Image Processing Lab at UC Berkeley recently developed an indoor mapping platform, which is described in [ $\left.\mathrm{LCC}^{+} 10\right]$. Their goal is to reconstruct a complete 3D model of building interiors including staircases. Hence, a backpack based platform was designed and consequently all six degrees of freedom have to be estimated, which is significantly more complex then the three degrees of freedom required when using a trolley. The orientation and translation estimation is based on four $360^{\circ}$ laser range finders and an inertial measurement unit. Nevertheless, the drift, especially in staircases, is comparably large and a loop closure is required to allow for reasonable mapping performance. Closing loops, however, usually increases the mapping time for a given building as some hallways have to be traversed twice. Finally, due to the additional degrees of freedom, motion blur is added by the gait specific movements.

Instead of designing the mapping platform around the requirements of approximately $10 \%$ of building interiors, it is preferrable to obtain the best mapping performance for $90 \%$. Thus, the upcoming mapping platform should again be designed as a trolley that is light enough to be carried on staircases. Localization on staircases can be performed by registering the images into the map via Structure-from-Motion approaches.

Using the virtual view approach, described in Section 7.2, panoramic images will no longer be necessary for localization purposes. Thus, the heavy and expensive Ladybug3 can be replaced by five Canon EOS M cameras. This mirrorless interchangeable lens camera has a significantly higher photosensitivity. Hence, no illumination equipment has to be mounted on the trolley, which drastically reduces battery drain and therefore weight. An illustration of the proposed new camera layout is shown in Figure 7.7. In addition to the cameras pointed sideways, one further Canon EOS M is pointing upwards onto a parabolic mirror to record $360^{\circ}$ panoramas. While this is not required for localization, it will be useful for pixel exact 
alignment of the recorded images using SfM approaches and for navigation interfaces on the mobile device, showing the directions overlaid in a panorama.

The currently mounted two laser scanners should be replaced by lightweight three Hokuyo laser range finders. In addition to one horizontal scanner, mainly used for localization purposes, two vertically mounted scanners, each rotated by $45^{\circ}$ to the side, incrementally construct a model as the trolley is pushed through the hallways. With two laser scanners twisted by $90^{\circ}$ to each other, significantly less occlusions can occur, which is important to build a detailed model of the interior. 


\subsection{Virtual View generation}

To overcome the limitations of the system architectures presented in the above chapters, the virtual view based approach is introduced in this section. As described above, estimating the location of the mobile device by determining the visually most similar geo-tagged reference image, results in a resolution of position estimates that is limited to the mapping trajectory. Further, the limited affine and perspective invariance of feature descriptors is a severe problem, as a location can be recognized only if a reference image with a pose similar enough to the query image exists.

With the 3D point cloud of the environment (see Figure 7.1) recorded by the vertical laser scanner as part of the mapping, virtual reference views at arbitrary locations and orientations can be generated via image based rendering. Considering indoor navigation as the main application of this system, the precision of a location estimate has to be in the range of one meter. A higher precision, e.g. down to a $\mathrm{cm}$, as required in robotic applications, is actually not necessary for LBS to be perceived as useful. The same is true for orientation estimation, where a resolution of around $20^{\circ}$ is sufficient for most applications. Hence, virtual reference views are placed every meter and at 16 orientations at every location, as illustrated in Figure 7.8. This allows us to determine the pose of the query image far of the mapper trajectory solely based in image retrieval results. Further, with reference images densely sampling possible locations and orientations, the visual similarity between the query image and the spatially closest database image is increased, which significantly improves retrieval performance. Due to the efficient inverted file structure of the image retrieval engine, the increased number of reference images does hardly increase the computational complexity.

The generation of photo realistic virtual views involves image based rendering techniques. There exists extensive literature in this research field, in which most approaches base upon complex 3D models of the environment $\left[\mathrm{LCC}^{+} 10\right]$. In the case of indoor environments with

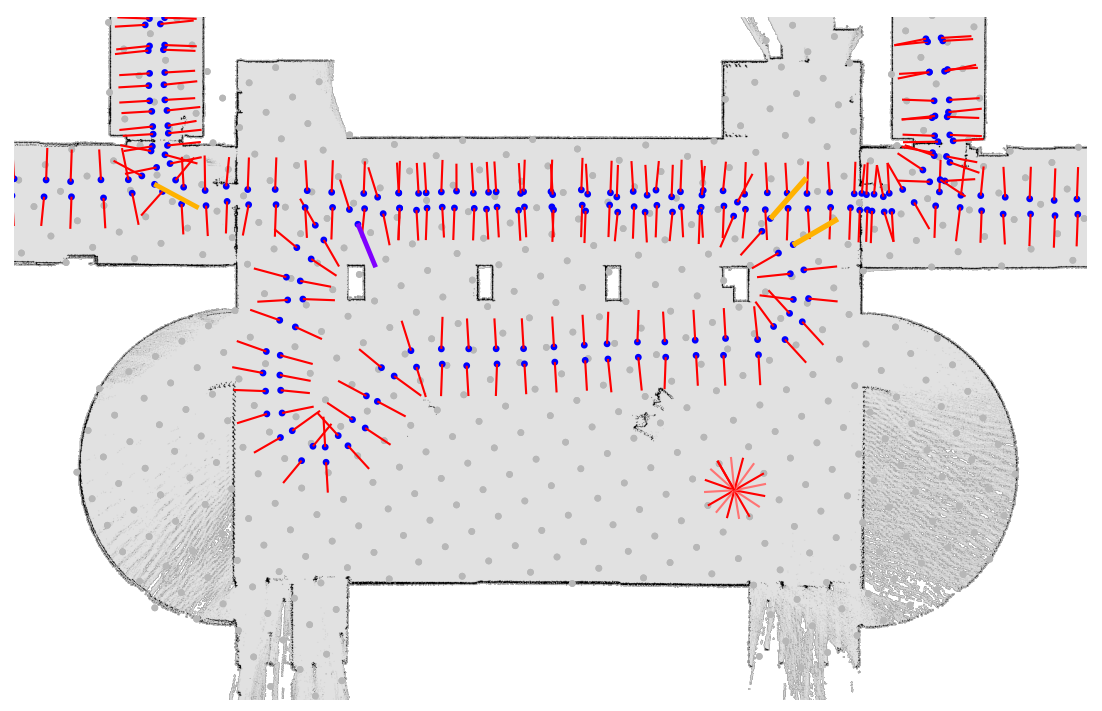

Figure 7.8: Images captured during mapping (blue dots, heading in red) and the virtual viewpoints created (grey dots). At each location, 16 views are computed (red compass rose). (Reproduced from [HSH $\left.{ }^{+} 12 b\right]$, (C) 2012 IEEE) 


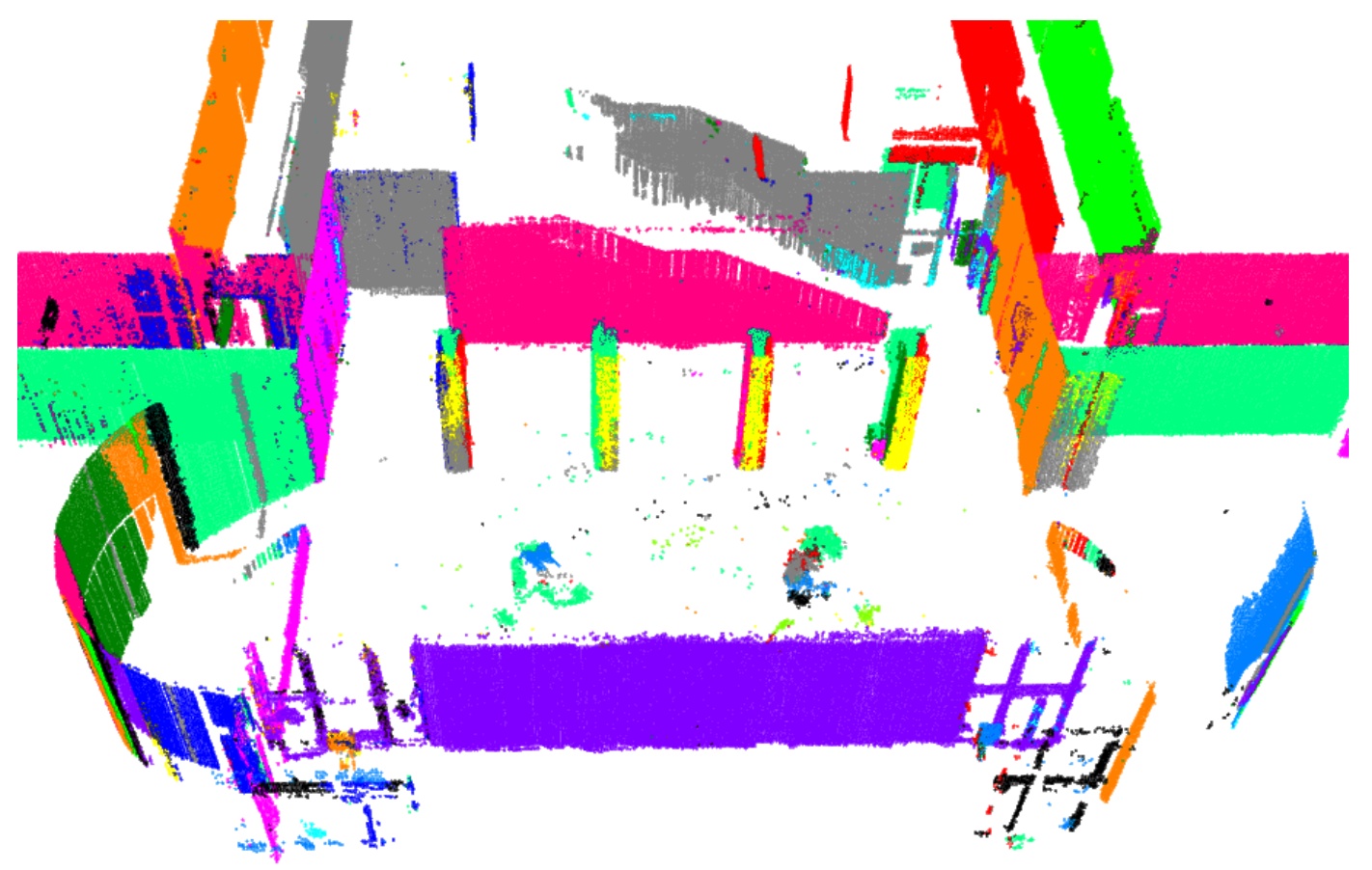

Figure 7.9: The point cloud acquired during mapping is segmented into planes (indicated by different colors) that provide the models for projective transforms. (Reproduced from [HSH+12b], (c) 2012 IEEE)

mostly planar structures, it might be sufficient to transform actually recorded images into the virtual views using planes detected in the point cloud as will be described in the following.

As a pre-processing step to generate virtual views, the 3D point cloud of the environment is segmented into planes as shown in Figure 7.9. In this figure, a different color has been assigned to each plane and the corresponding points in the point cloud are drawn. The ground and ceiling planes are not considered as usually only very few visual features can be found on them. Segmentation is performed by generating plane hypotheses based on the direction of minimum variance of points in a given vicinity. These hypotheses are validated by a minimum amount of points that lie on that plane. Points assigned to the plane are removed from the point cloud and the process iterates until a maximum number of points remain in the point cloud.

Based on this segmentation, for each virtual view, the visible planes are identified via ray casting. In a second step, all recorded images that cover a part of the visible planes are selected. To maximize the quality of the virtual view, only those images are used to generate the virtual view that provide a sufficient spatial resolution. Further, it is important to ensure that each used reference image covers a significant part of the virtual view, to reduce the number of artificially introduced borders. Due to the resolution of the point cloud, small details in the 3D structure of the environment can get lost. However, if the viewing directions of a reference image and virtual view are similar, the required transformations are small and thus, the errors are less pronounced. Hence, reference views with similar viewing direction to the virtual view are preferred. 


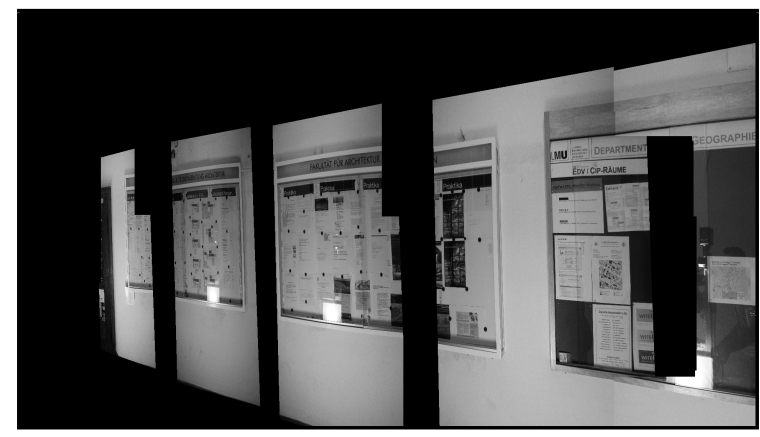

(a)

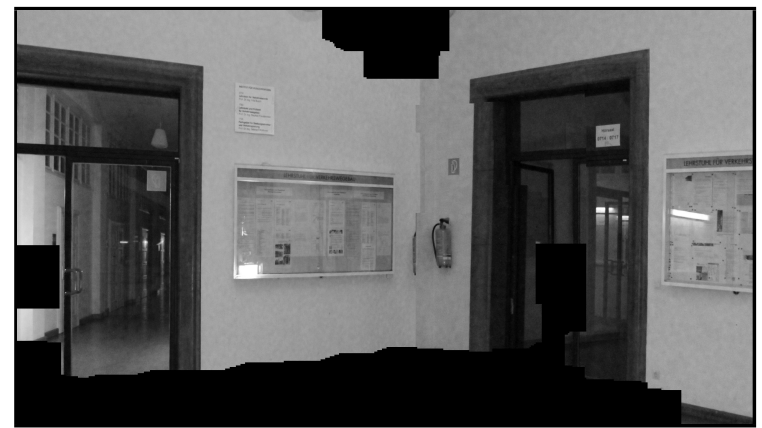

(b)

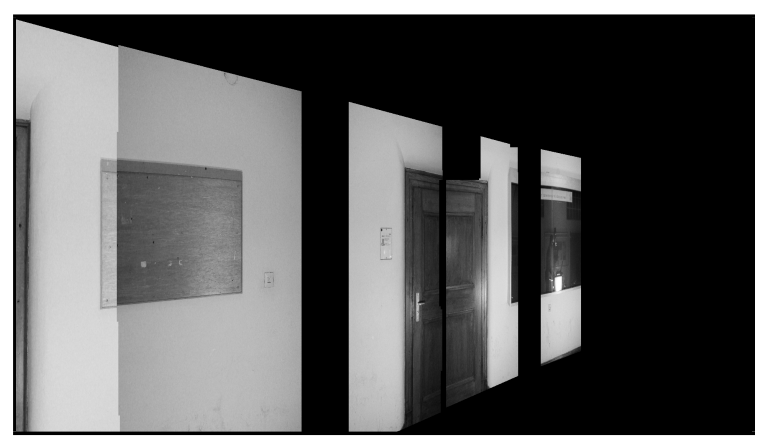

(c)

Figure 7.10: Exemplary virtual views at three different locations. Misalignment is caused by imperfect calibration of the cameras as well as localization errors. Features are extracted only on the warped image patches and not on artificial borders.

To warp a reference image into a virtual view, a homographic transformation has to be determined, which is discussed in the following. The camera pose of the reference image is denoted by a homogeneous $4 \times 4$ matrix $\mathbf{T}_{r e f}$, the pose of the virtual image is denoted by $\mathbf{T}_{\text {virt. }}$ The relative transformation between both views follows as

$$
\mathbf{T}=\mathbf{T}_{\text {ref }}^{-1} \cdot \mathbf{T}_{\text {virt }}=\left[\begin{array}{ll}
\mathbf{R} & \mathbf{t} \\
\mathbf{0}^{T} & 1
\end{array}\right] .
$$

Here, $\mathbf{R}$ is the three dimensional rotation matrix and $\mathbf{t}$ is the three dimensional translation vector that relates the reference and virtual view. With a plane defined in Hessian normal form $\mathbf{x}^{T} \cdot \mathbf{n}=d$, the distance between the plane and the reference image is

$$
\Delta=\left|\mathbf{T}_{r e f}^{T} \cdot \mathbf{n}-d\right| .
$$

The homography $\mathbf{H}$ relating coordinates in the reference image to coordinates in the virtual image is then given by

$$
\mathbf{H}=\mathbf{K}_{\text {virt }}\left(\mathbf{R}-\mathbf{t} \cdot\left(\mathbf{T}_{\text {ref }}^{-1} \cdot \mathbf{n}\right)^{T} \cdot \frac{1}{\Delta}\right) \mathbf{K}_{r e f}^{-1},
$$

where $\mathbf{K}_{r e f}$ and $\mathbf{K}_{\text {virt }}$ are the camera calibration matrices for the reference image and the virtual image, respectively. 
Using Equation 7.3 , the reference image is warped to the virtual viewpoint and local image features are extracted from the resulting image patch. For any non-trivial scene, the generated patch contains areas that are not part of the considered plane. For this reason, all features outside the corresponding pixel mask are discarded. Figure 7.10 shows three exemplary virtual views. Misalignments between the individual warped image patches are caused by imperfect calibration of the cameras as well as localization errors. Especially errors in the orientation of the reference images are amplified in the transformation. A structure from motion approach to allow for pixel accurate calibration and reference image localization should be applied in the future.

Finally, the features extracted from all the planes in a virtual view are combined into a single bag-of-features vector that is indexed by a CBIR system for retrieval during localization.

\subsection{Integration of Binary Features}

With the proposed virtual view based approach, providing a reference view at every meter and at 16 orientations each, it can be assumed that a high invariance of feature descriptors with respect to affine transformations is no longer required. Rather the distinctiveness is reduced by the invariance. Further, with eliminated network latency, low complexity quantization (see Chapter 5), the major computational bottleneck can be found in the feature extraction.

Recently, several low complexity features have been proposed CLSF10, RRKB11, LCS11, AOV12, which all have in common that a binary vector is used to describe an image patch around an interest point detected by the FAST [RD06] feature detector. The Oriented FAST and Rotated BRIEF (ORB) [RRKB11], the Binary Robust Invariant Scalable Keypoints (BRISK) [LCS11, as well as the Fast Retina Keypoint (FREAK) [AOV12], are derivatives of the Binary Robust Independent Elementary Features (BRIEF) [CLSF10. While BRIEF is the least complex and hence fastest to extract descriptor (approximately 40 times fast than SURF), ORB, BRISK, and FREAK provide an improved invariance with respect to affine transformations.

Thus, it stands to reason to evaluate the performance of BRIEF in combination with the proposed virtual reference view approach. The BRIEF descriptor is computed by performing a series of image intensity comparisons within the image patch. The positions for those comparisons are sampled from a normal distribution once and are then fixed for all descriptor extractions. Figure 7.11 shows the image intensity comparison pattern used for the BRIEF descriptor extraction in the experiments in Section 7.4. For each comparison an entry is added in the binary descriptor vector.

A smoothing using a Gaussian kernel of size $9 \times 9$ pixels with a variance of 2 pixels is performed on the patch to enhance the robustness against small errors in the interest point detection. This smoothing can be done efficiently using integral images. While theoretically any number of comparisons and thus number of bits $D$ for the BRIEF descriptor would be possible, Calonder et al. [CLSF10] found that $D=256$ offers a good trade-off between recognition rates and storage efficiency. Therefore, in this work the 256 bit version of BRIEF is used. Further, compared to SURF, which uses 512 bit (quantizing each dimension to 8 bit), this is a significant reduction in terms of memory consumption. 


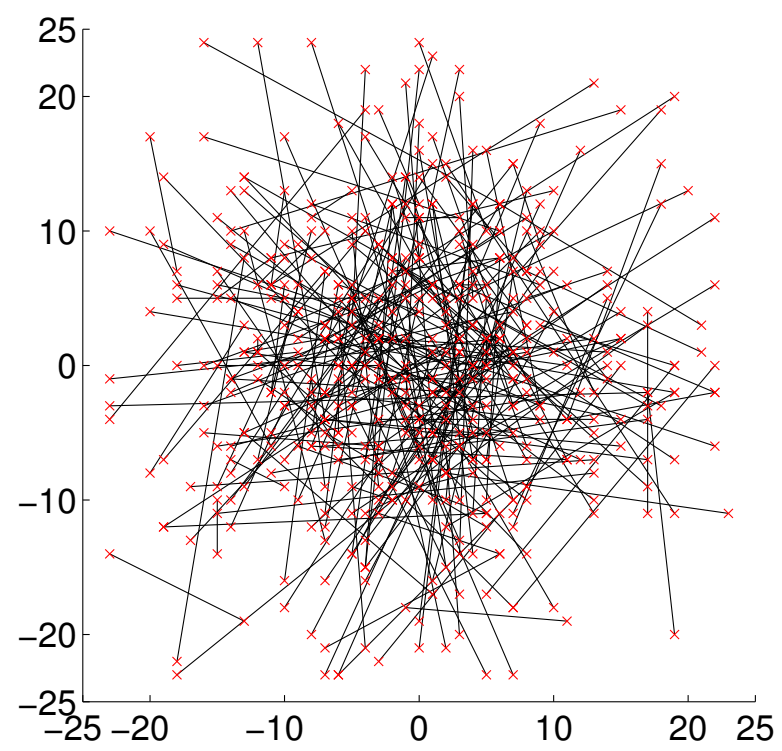

Figure 7.11: Intensity comparison pattern of BRIEF

As the comparison positions are sampled independently from a normal distribution, bits in the descriptor are independent and their ordering is arbitrary. This means the BRIEF descriptor is a point in 256 dimensional Hamming space. Hence, the Hamming distance can be used as a similarity measure between two BRIEF features. This distance measure is defined as the number of bits in two bit strings that differ. This is an important property as it allows us to apply locality sensitive hashing (LSH) [DIIM04 to perform an approximate nearest neighbor search.

To apply binary features to the existing image retrieval framework described above, the k-means algorithm needs to be adapted. K-means clustering, as described in Section 2.2.2, is applied to determine centroids, called visual words, in the high dimensional descriptor space, that best describe all detected features in the training dataset. In a first step, $K$ random centroids $\mu_{\mathbf{k}}$ are chosen to initialize the iterative k-means algorithm. Second, the training data is separated into $K$ sets $P_{k}$ by assigning all features $\left(\mathbf{x}_{\mathbf{j}}\right)$ to their closest centroid:

$$
P_{k}=\left\{\mathbf{x}_{\mathbf{j}}:\left\|\mathbf{x}_{\mathbf{j}}-\mu_{\mathbf{k}}\right\|_{H}<\left\|\mathbf{x}_{\mathbf{j}}-\mu_{\mathbf{i}}\right\|_{H}\right\} ; i=1 \ldots K, i \neq k
$$

Third, based on the subsets $P_{k}$ new centroids are computed as the means of the set $P_{k}$. This process iterates until the mean distance between the centroids and their set members does no longer decrease.

However, in the binary descriptor space of BRIEF it is hardly possible to compute a mean to determine the updated centroid. Thus, instead, a voting approach is proposed in [Str12]. Every feature in a cluster votes for bit positions which are ones in its own bit string. These votes are accumulated in one scoring vector with elements score $_{i}$ for each bit position: 


$$
\text { score }_{i}=\left\{\begin{array}{rl}
\text { score }_{i}+1 & \text { if bit } \\
\text { score }_{i}=1 & \text { otherwise }
\end{array} .\right.
$$

A threshold of half the number of features $N_{C l}$ in the cluster is applied to the resulting votes score $_{i}$ to obtain a binary string for the centroid:

$$
\text { bit }_{i}=\left\{\begin{array}{ll}
1 & \text { if } \text { score }_{i}>N_{C l} / 2 \\
0 & \text { otherwise }
\end{array} .\right.
$$

This voting approach comes up with the same result as the naive implementation which would convert the binary vectors to a floating point number, compute the mean, round and back convert the resulting centroids. Clearly, the voting strategy needs less computational effort and memory than the naive implementation.

In order to facilitate the above described binary k-means (BKM) clustering of large datasets in the order of $10 \mathrm{M}$ to $100 \mathrm{M}$ features into about $200 \mathrm{k}$ clusters an exhaustive search for the closest centroid needs to be replaced by an approximate nearest neighbor (ANN) search. As mentioned above, the similarity between two BRIEF descriptors can be efficiently computed using the hamming distance, which allows us to apply Locality Sensitive Hashing (LSH) to assign each feature to the approximately nearest centroid.

In order to tune the parameters of LSH for the binary k-means clustering, the performance of matching descriptors to 200k centroids is evaluated. The results were collected on an Intel(R) Xeon(R) CPU X5660 running at 2.80GHz. For each parameter configuration 20 different randomly sampled query sets of 100 BRIEF features each are matched against 200k centroids obtained by BKM clustering of a virtual views dataset.

In Figure 7.12 four major properties of the LSH are evaluated with respect to the hash function length $m$ and the number of hash functions $l$. The computation time per query, the probability of finding the true nearest neighbor, as well as the percentage of query features that actually find a match at all are plotted in Figures 7.12a, 7.12b, and 7.12d, respectively. In the case of long hash functions, only very few nearest neighbor candidates are returned. Ultimately, no candidate at all might be returned and thus also no matching centroid can be found.

As the quantization structure should easily fit into the RAM of a mobile device, the number of hash tables and thus the memory requirements has to be considered as a constraint. Clearly, the optimal LSH configuration would result in maximum precision and minimum computation time. By looking at the joint plot of timing and precision performance in Figure 7.12c), $m=19$ and $l=31$ is found to be a good parameter set. This configuration has $90 \%$ retrieval precision at matching times of about $560 \mu$ s per query. Compared to $5.3 \mathrm{~ms}$ for exhaustive NN search, this presents more than a nine-fold speedup. The contour line corresponding to exhaustive nearest neighbor search is overlaid in red for easy comparison. The memory footprint of the 31 hash tables storing 200k centroids each amounts to $138.8 \mathrm{MB}$.

In contrast to SURF that uses at least 8 bits per dimension, it is important to note that binary features represent points in a highly quantized space. This significantly increases the probability, that two features have exactly the same distance to a third feature. This is considered in the image retrieval framework by scoring for all centroids, i.e., visual words, that share the same minimum distance to the query feature. 


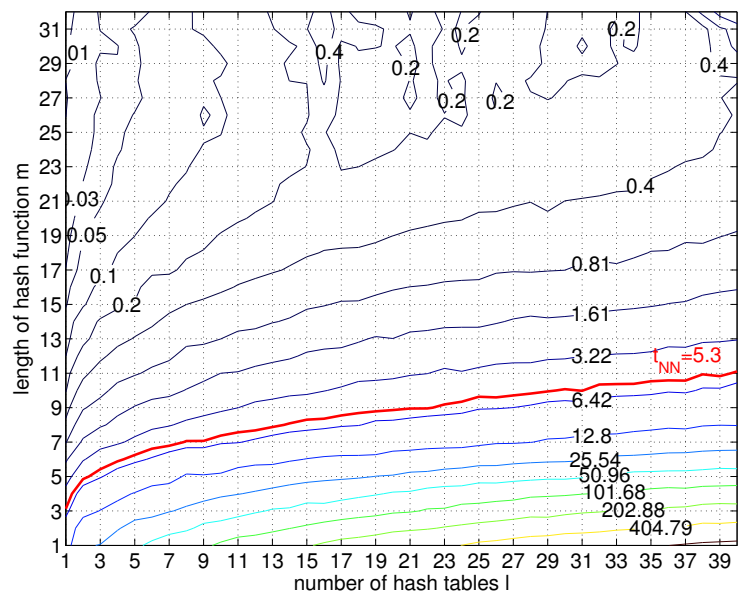

(a) LSH timing per query $[\mathrm{ms}]$

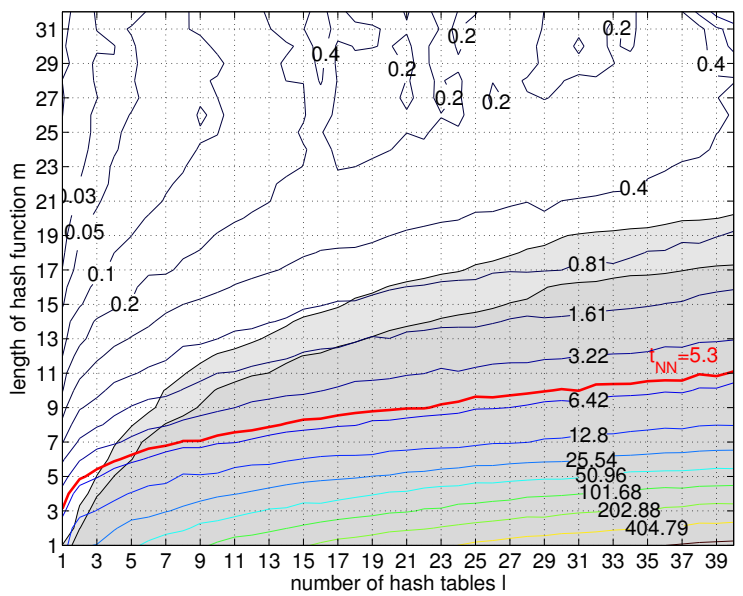

(c) LSH timing per query [ms] with $90 \%$ and $95 \%$ precision areas

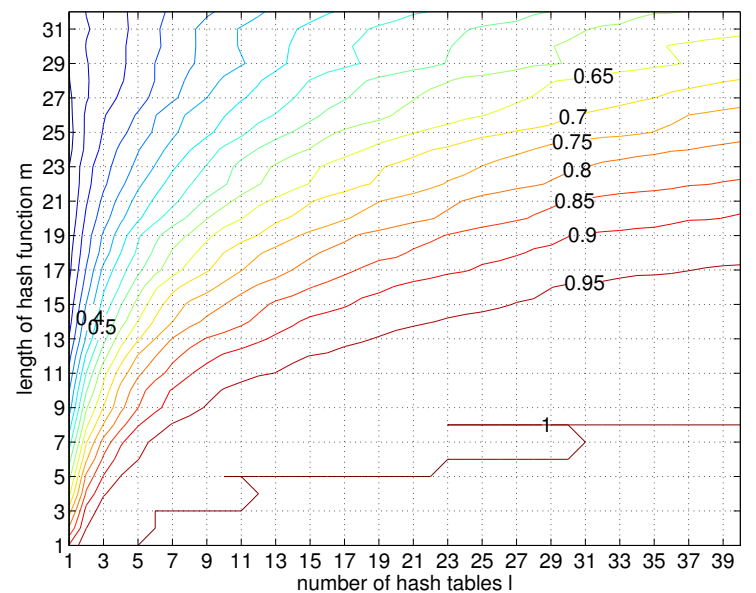

(b) LSH precision

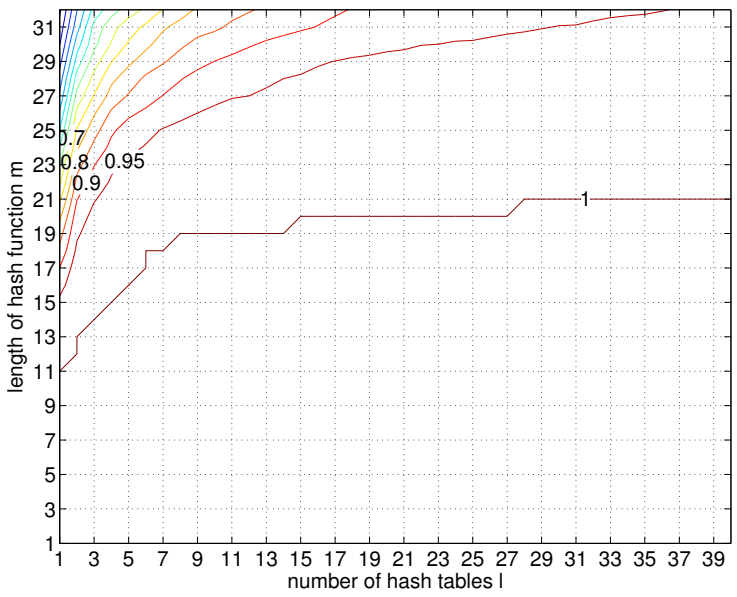

(d) LSH percentage of matched features

Figure 7.12: Timing, precision and percentage of matched features for LSH over $m$ and $l$. The timings are given for the LSH search of the approximate $N N$ of one feature in the database. The contour line corresponding to the timing of exhaustive NN search is plotted in red to allow for easy comparison.

Finally the binary k-means clustering seemlessly integrates with the low latency approach proposed in Chapter 5. Employing SURF as a feature descriptor requires the use of a randomized forest of kd-trees to efficiently quantize features to visual words (kd-tree was shown to perform best among state-of-the-art ANN algorithms). However, randomized kd-trees adapt, although only to a limited extend, to the database statistics. Hence, with changing partial vocabularies, also the kd-trees need to be updated from time to time as mentioned in Chapter 5 .

This is different with LSH that replaces the forest of randomized kd-trees in the case of binary features. The hash functions are completely random and thus not dependent on the database. Further, with BRIEF features requiring only half the memory of SURF, the network load for the client server connection is reduced. 


\subsection{Localization Results}

The performance of the virtual view based indoor localization approach, is evaluated using the publicly accessible image and point cloud TUMindoor dataset $\left[\mathrm{HSH}^{+} 12 \mathrm{a}\right]$, described in Section 7.1. A simple scheme is used to determine locations where virtual views are created. The floor plan is sub-sampled to a resolution of one meter per pixel, and a virtual location is created for each "free" pixel. The height of the virtual camera is fixed at $1.50 \mathrm{~m}$ above ground. In total 101,632 views at 6,352 locations are generated.

First, the approach is evaluated using the widely adopted SIFT features, which provide invariance with respect to scale, rotation and are robust against affine transformations. The image retrieval system is trained on 24.8 million SIFT features extracted from the image patches from the virtual views. An approximate k-means (AKM) quantizer with a vocabulary size of 200,000 visual words and TF-IDF weighting is used. The query time per image on a single thread is around $200 \mathrm{~ms}$, however AKM can easily be configured to perform considerably faster.

The system is queried using images captured at various locations in the mapped environment. To demonstrate that the system is capable of inferring the appearance at arbitrary locations, attention is paid to keep distance to the mapper trajectory. Four query images and the corresponding results are shown in Figure 7.13. The proposed method is able to robustly determine the correct orientation (quantized to intervals of $\frac{\pi}{8}$ ). The top-ranked image retrieval results concentrate in the immediate vicinity of the query location in almost all cases, only the rank-2 location result for the lower-right query image is a bit off. Nevertheless, the orientation is determined correctly in all cases.

The virtual camera is using the same calibration matrix as the query camera to ensure that the virtual views match what the query camera would see. If the field of view (FOV) between the cameras differs too much, a localization error along the camera's z-axis occurs. For the application of mobile device localization, it can be assumed that the FOVs don't vary too much between phone models. Further, the focal length of query cameras may be artificially lengthened simply by cropping the region of interest for feature extraction.

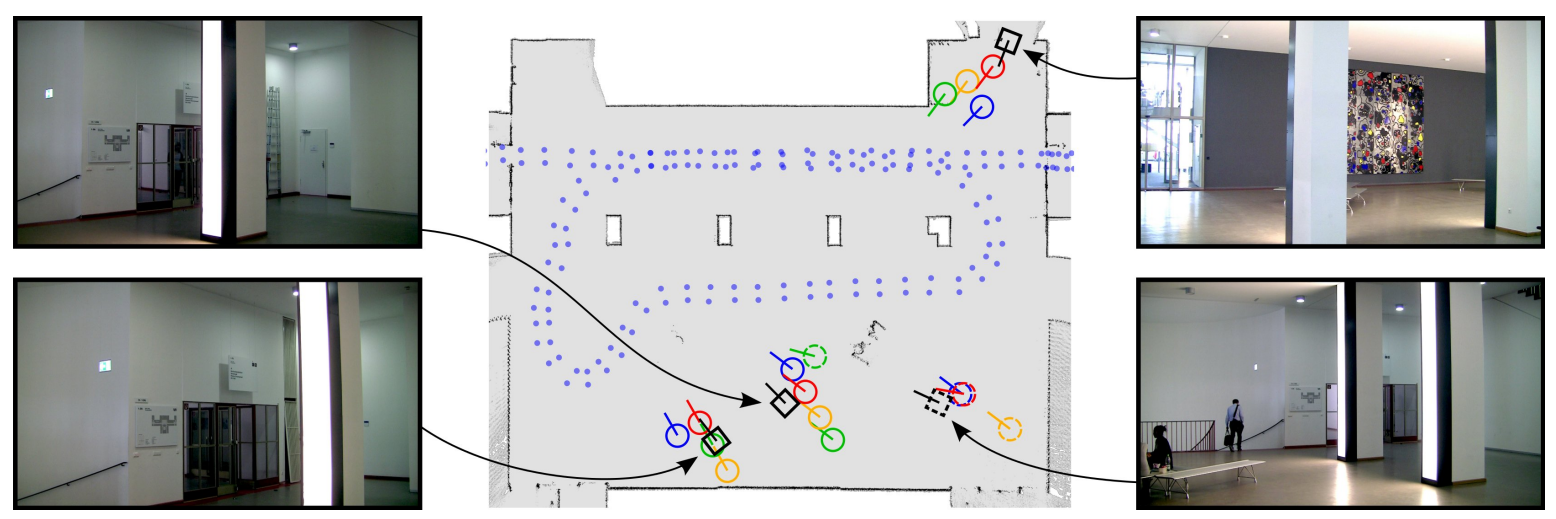

Figure 7.13: Top-ranked retrieval results for 4 query images (black square is the ground truth pose). Location and orientation are drawn as circles (rank 1: red, 2: green, 3: blue, 4: orange). No post-processing of image retrieval results has been applied. (Reproduced from [HSH $\left.{ }^{+} 12 b\right]$, (c) 2012 IEEE) 


\begin{tabular}{r|c|c|c} 
& P @ 1 & P @ 3 & P @ 5 \\
\hline Reference Views $(r=5 m)$ & 0.33 & 0.28 & 0.25 \\
Virtual Views $(r=3 m)$ & 0.46 & 0.43 & 0.41 \\
Virtual Views $(r=5 m)$ & 0.57 & 0.57 & 0.56 \\
\hline
\end{tabular}

Table 7.2: Mean precision at cutoff ranks 1, 3 and 5. Relevant views are within radius $r$ around the query location.

Table 7.2 shows the mean precision over 252 queries (six frames at 42 locations) achieved by the first result, by the top-3 results, and by the top- 5 results, respectively. A precision of 1.0 is achieved if all top-ranked results are relevant. Clearly, the virtual view approach outperforms the unprocessed reference images. In $57 \%$ of all cases, the top-ranked result is a correct location with our virtual view approach, compared to $33 \%$ when only reference images are used.

In a second step the performance of virtual views in combination with the low complexity BRIEF features is evaluated. As described above, the binary BRIEF feature descriptor does not provide invariance with respect to scale and view point changes. Further, BRIEF employs the FAST interest point detector, which offers a low repeatability compared to the more complex SIFT and SURF features. While an invariance to strong view point changes can be assumed to be no longer required in the virtual view based approach, we still require a high probability, that the same interest point is detected in matching images.

To achieve this, the interest point candidates returned by the FAST feature detector are evaluated using the Shi-Thomasi cornerness measure [ST94]. This measure evaluates the interest points using the eigenvalues of the surrounding intensity gradients and thus allows us to select the most stable and thus repeatable features. This post-selection can be perceived as an augmentation of the feature detection process, which increases the repeatability with very little additional computational complexity.

At each detected interest point, BRIEF describes an image patch of size $48 \times 48$ pixels. As mentioned in Section 2.2.1, the size of the image patch used to generate a descriptor is a tradeoff between the distinctiveness and the robustness against occlusions and overlaps in the $3 \mathrm{D}$ scene. In this experiment, BRIEF features are extracted on a resolution pyramid, which includes the virtual view in its original resolution and at two additional resolutions downsized by factor of 0.5 and 0.75 . At these low resolution versions an image patch of $48 \times 48$ pixels covers an enlarged area of the scene geometry. With BRIEF descriptors covering small details and broad areas, the resolution pyramid results in complimentary features.

The quantization of extracted BRIEF descriptors into visual words is performed using the proposed binary k-means clustering with Locality Sensitive Hashing (LSH) as an approximate nearest neighbor algorithm. For LSH, 31 hash tables with a length of 19 bits each are used as described above. The size of the visual vocabulary is again set to 200,000 visual words, which proofed to be a good compromise between discriminative power and probability of correct quantization.

To compare the performance of the approach when applying SIFT and BRIEF features respectively, the mean precision scores are given in Table 7.3. It is important to note that the random initialization of the k-means algorithm and the approximate nearest neighbour search lead to small performance variations. Thus, only the first two digits of the precision score 


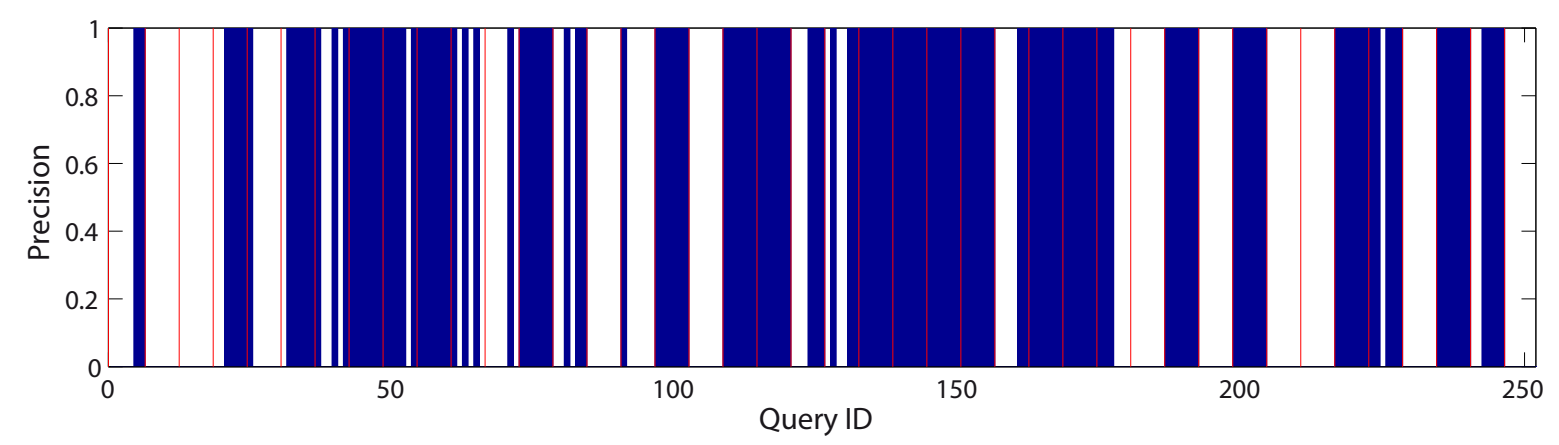

(a) Precision@1

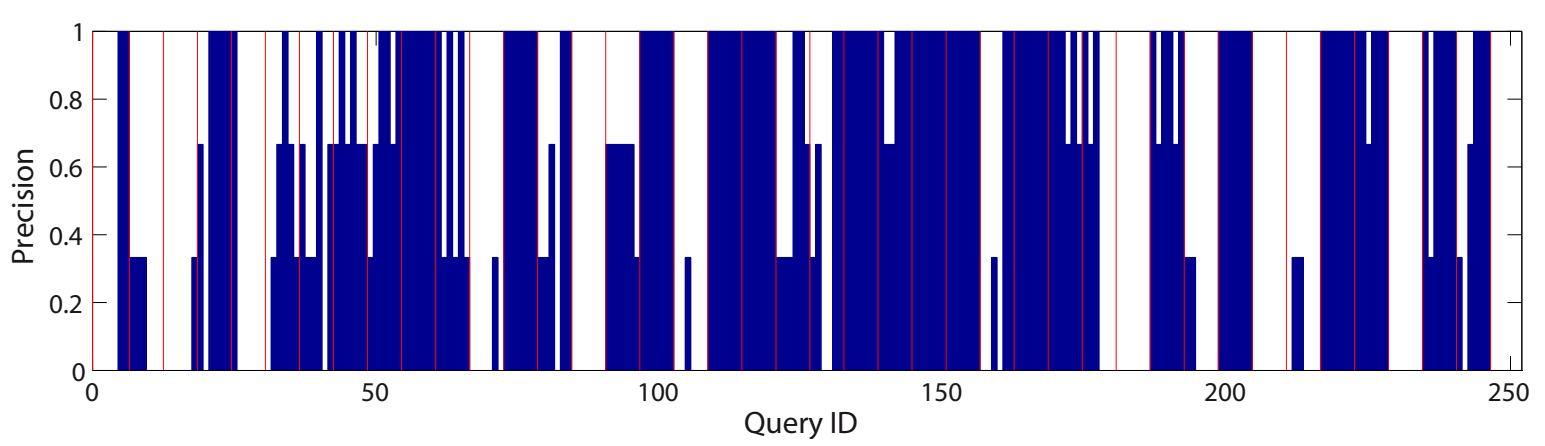

(b) Precision@3

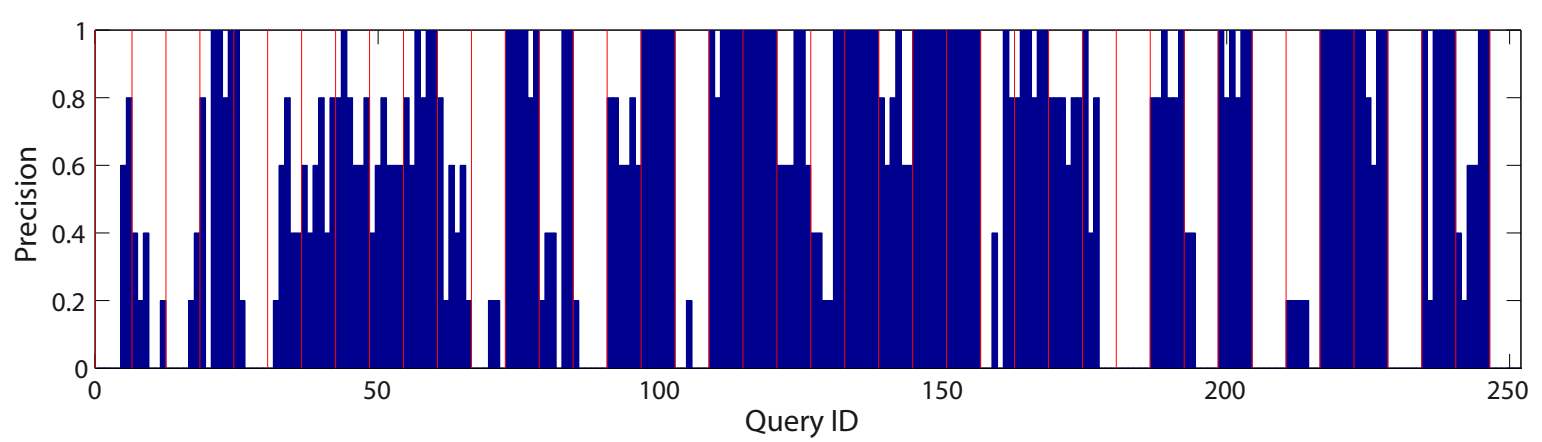

(c) Precision@5

Figure 7.14: Precision score for each individual query with respect to the first ranked retrieval result, the top-3, and top-5 results respectively. The precision and thus the percentage of retrieved relevant results can be either 1 or 0 when considering the first ranked result only. 


\begin{tabular}{r|c|c|c} 
& P @ 1 & P @ 3 & P @ 5 \\
\hline Virtual Views (SIFT) $(r=5 \mathrm{~m})$ & 0.574 & 0.571 & 0.562 \\
Virtual Views (BRIEF) $(r=5 \mathrm{~m})$ & 0.572 & 0.579 & 0.556 \\
\hline
\end{tabular}

Table 7.3: Comparison of SIFT and BRIEF feature based location recognition. Mean precision scores are provided at cutoff ranks 1, 3 and 5. Relevant views are within $5 \mathrm{~m}$ around the query location.

should be considered. With the results in Table 7.3 , it can be concluded that, despite the lack of invariance with respect to view point changes, the BRIEF feature based approach achieves approximately the same performance as the significantly more complex SIFT features. This confirms the assumption that dense virtual reference views reduce the invariance requirements on feature descriptors. In fact, the lack in invariance of BRIEF features adds additional distinctiveness as described above.

To evaluate the results in more depth, the precision score for each individual query is shown in Figure 7.14. The precision score is the percentage of correct locations (those within a radius of $5 \mathrm{~m}$ around the ground truth) among the top-1, top-3, or top-5 results, respectively. When considering the first ranked result only (precision@1), the precision and thus the percentage of retrieved correct results can be either 1 or 0 . The used query frames and their IDs are illustrated in Figure 7.5 to allow for a comparison with the results. At each location, 6 query frames are used. Groups of frames captured at the same location are indicated by the red vertical lines in Figure 7.14

When examining the results for frames 103 - 108, the performance is very low and hardly any correct locations can be found among the top- 5 results. This is caused by the fine structured geommetry and glass surfaces. The current virtual view rendering requires large planes to warp reference image into the virtual view. In case of small planar patches, artificial borders are introduced in the virtual view generation, which affect the detected features. Nevertheless, the assumption of mostly planar structures in indoor environments holds. Query frames 109-114, capturing a complex three-dimensional and sparsely textured scene, result in an impressive location recognition performance. Almost all returned locations are within $5 \mathrm{~m}$ around the ground truth location.

\subsection{Summary}

This chapter introduces the novel virtual view approach, which allows for a meter level localization accuracy and orientation assignment in fractions of a second. In addition to actually recorded reference images, virtual reference views at every meter and at 16 orientations are added to the database. By matching the current query image to the virtual view database, the location and orientation of the mobile device can be directly determined without the need for computationally complex post-processing approaches. The rendering of photo realistic virtual views requires information about the 3D geometry of the scene, which is recorded by the introduced mapping platform using a laser range finder.

With reference images densely sampling possible locations and orientations, the visual similarity between the query image and the spatially closest database image is increased. Thus, the virtual view approach does not only allow us to determine camera pose far off the mapper 
trajectory but also leads to a significantly improved retrieval performance.

Further, it can be assumed that a high invariance of feature descriptors with respect to view point changes is no longer required but rather reduces their distinctiveness. Therefore, the low complexity Binary Robust Elementary Features (BRIEF), which provide hardly any invariance with respect to affine transformations, are integrated into the location retrieval framework. To this end, a Locality Sensitive Hashing based quantization structure is introduced. Experimental evaluation shows that with dense virtual reference views, BRIEF features can match the performance of SIFT features despite the lower repeatability and no invariance with respect to view point changes. With eliminated network latency, low complexity quantization (see Chapter 5), feature extraction poses the major computational bottleneck of the current system architecture. With feature extraction times 40-fold faster than SURF, the computational complexity at the mobile device can be significantly reduced. 


\section{Conclusions and Outlook}

With the ever increasing interest of global companies, research institutes, and various new start-ups, it can be well assumed that indoor positioning technology will be a major innovation in next generations' mobile devices. While most existing positioning solutions rely on a costly infrastructure, which states a major hurdle for a widespread adoption, this thesis presents a novel vision based approach. Image recordings of the current environment are used as a visual fingerprint and matched to a previously recorded and geo-tagged reference database. This basic concept, however, implicates several challenges which are addressed in this thesis.

The ability to recognize the location and orientation within fractions of a second is a fundamental design criterion. A user needs to know his position in the very moment he pulls out his smart phone. To this end, the proposed system is based upon content based image retrieval techniques, which are typically applied to product recognition tasks. With complex three-dimensional scenes, clutter in the field of view, reflections on windows and show cases, the location recognition task is complicated. This thesis introduces several concepts to cope with the associated challenges. A high level feature selection to exclude unreliable features in both the query as well as in the database is shown to be of major importance for the retrieval performance. Virtual feature panoramas, combining the information of multiple consecutively recorded frames, virtually increase the field of view and thus allow us to distinguish visually similar locations.

In mobile visual location recognition a client server architecture is required, which minimizes the transmission delay and thus the overall system latency. This thesis proposes an approach that eliminates the network delay by preloading those parts of a flexible location recognition engine to the mobile device that are required to perform a localization within a limited area. Hence, for a given time period, localization can be performed locally without waiting for responses from the server. Preloading information to the mobile device can be very efficient due to the typically 5 times faster downlink. Further, retrieval performance is significantly increased by identifying relevant parts of the recognition engine based on the uncertainty about the location of the client. This way, prior knowledge is efficiently integrated into the image matching process.

Text, a major source of information for humans to localize and navigate, is exploited to cope with sparsely textured indoor environments. With today's camera phones, however, the resolution is hardly sufficient for reliable text recognition. Thus, a novel approach is developed that requires text to be detected and localized only. This allows us to integrate the information on the arrangement of the characters into an appearance based matching process, which compares the recorded text to previously recorded signs, posters, and others, to reveal the current location. The resulting increase in distinctiveness enables applications like a door sign recognition which reliably determines the location in fractions of a second without the use of error-prone optical character recognition.

Meter level localization and orientation estimation is essential, when it comes to navigational and augmented reality applications. Reference images, however, cannot be recorded at every 
possible location and orientation to keep the mapping of a building in reasonable time limits. Thus, virtual reference views are introduced, which are computed at every meter and at 16 orientations via image based rendering techniques. This novel concept allows for positioning at places where no reference images have actually been captured. Further, the dense sampling of views within an environment relaxes the requirements of the image descriptors with respect to their invariance against view point changes. This in turn allows for the use of low complexity visual features which reduces the overall system latency.

The mobile visual location recognition system presented in this thesis is designed to work on today's smart phones. Low complexity visual features and retrieval algorithms are not only required to minimize the system latency but also to reduce battery drain. This is especially important, when considering the use of visual positioning in augmented reality interfaces like the upcoming Google Glass Goo12b. With these camera integrated glasses the use of visual data as the basis for localization systems seems almost obvious.

The experimental results of this thesis show that vision based mobile indoor localization is feasible under certain assumptions which form the basis for future research directions. These can be subdivided into the major components of the visual localization and mapping system.

This thesis mainly concentrated on the image retrieval based global position and orientation estimation. While the concept of virtual reference views proofed to be an important step towards meter level localization and orientation estimation, up to now, only planar parts like walls are reconstructed in the artificially created views. To improve the performance of the localization system, it will be important to enhance the quality of the computed virtual views. Computing a complete, photo realistic view at arbitrary locations is a tough problem and several approaches exist in the active research field of image based rendering. It can be assumed, that approaches, which do not require a complex and fine structured 3D model of the environment but just an approximation will be superior in their application to visual localization.

In the current state of the localization system, a number of distinct visual cues are required to determine the current location. In locations like hospitals with alike looking plain corridors, it will be necessary to capture the geometric relation among the features and to integrate them into the matching process. With enhanced virtual views and the resulting dense sampling of references in the environment, global instead of local image descriptors should be investigated to include these geometric relations. This cloud allow us to cope with very sparsely textured environments.

In addition to enhancements to the global visual localization, new concepts for a robust visual odometry need to be conceived. Under certain assumptions (e.g., abundance of visual features, static environment, smooth motion), state-of-the-art visual odometry systems achieve remarkable efficiency as well as accuracy. In real world application, however, it is inevitable that these assumptions are violated which causes tracking failures. With the assumption of salient visual features comes the requirement that the user is forced to hold up the smart phone at any time, which can be very inconvenient. Thus, new concepts should allow the user to hold the device in an accustomed manner, i.e., the camera is pointing towards the floor, for most of the time. With the floor as a single planar structure to be tracked from an approximately constant height and known orientation very efficient algorithms can be designed to exploit the limited available information. Fusing this information with a pedometer, floor plans, and signals of opportunity like WiFi, could allow for a robust relative 
pose estimate. As the proposed indoor navigation system is designed to assist persons to find their way from A to B, the major goal of relative pose estimates is to determine whether he or she is still on the right track or took a wrong turn. This stands in contrast to the primarily robotic applications of visual odometry where a precise position is required. Addressing specifically this difference may allow us to identify novel approaches.

As no infrastructure is required in the case of vision based indoor localization systems, the rate of adoption of is mainly driven by the ease of the mapping process. Hence, no expert knowledge, no expensive hardware should be required and the mapping of a building structure should take a minimum amount of time. Until now, the mapping trolley developed as part of the NAVVIS project is still cumbersome, heavy and the costs for the used laser scanners are comparably high. The upcoming version, described in Section 7.1.1 will be significantly more flexible, of lower weight, and allow for the mapping of difficult to access areas. Further improvements, however, can be achieved by removing laser scanners and perform purely vision based SLAM to determine the image locations. While autonomously driving trolleys would render a human operator unnecessary, this, however, would require the robotic trolley to also be able to open doors. It might be more reasonable to allow users to map small buildings on their own using their smart phones. With increasing quality of build-in cameras, the recorded videos could be pre-processed on a local pc and results uploaded to a server. This would ultimately allow for a ubiquitous availability of vision based localization.

Further, the visual appearance of building interiors is typically not static but gradually changing over time. New posters, furniture, and others are added and removed. As an intermediate step to a completely crowd sourced mapping of buildings, images, recorded by the users to localize themselves, should be used to also update the database. This would allow us to avoid mapping a building over and over. This further allows for adapting to the environment and to weight reliable visual cues.

While there are still challenges that need to be addressed in future work, the developed system has been shown to allow for a meter level localization and orientation estimation under realistic conditions at its current state. Thus, it stands to reason, not only to continue research, but also to advance the system to a commercial product. 


\section{List of Figures}

1.1 Track of video recordings in downtown San Francisco. Ground truth is shown as a colored path (color corresponds to elevation level), whereas the GPS recordings of the iPhone 4 are shown in black. (Reproduced from [SHC $\left.{ }^{+} 11\right]$, (c) 2011

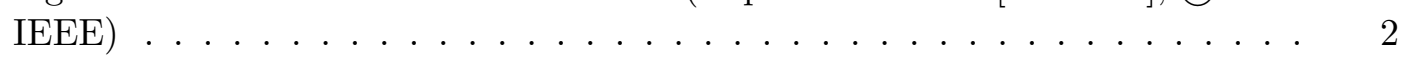

1.2 Mockup of a mobile visual localization system in an indoor environment. . . . 4

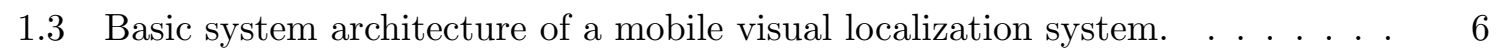

2.1 Left: A frame with feature points that are tracked by PTAM. The color of a point indicates the size of the feature in the image. Right: The corresponding three-dimensional point cloud as it appears in the reconstructed map. The 3D positions of the points are very noisy. The estimated ground plane is indicated by the grid lines. (Reproduced from $\left[\mathrm{HMH}^{+}\right.$12], (c) 2012 IEEE) $\ldots$. . . . . . . . 11

2.2 Google Street View panorama matched to a low resolution video recording (Union Square, San Francisco) using the MHVT-based location recognition system proposed in Chapter 3 . (Reproduced from $\left[\mathrm{SHC}^{+} 11\right]$, (c) 2011 IEEE)].

2.3 Sample images from the Google Street View dataset of San Francisco; available panoramas are on average $12.6 \mathrm{~m}$ apart from each other. . . . . . . . . . . . . 13

2.4 Sections of a Google Street View Panorama recorded in San Francisco. Yellow ellipses are fitted around detected MSER [MCUP04] regions. (Reproduced from $\left[\mathrm{SHC}^{+} 11\right]$, (c) 2011 IEEE) $] \ldots \ldots \ldots \ldots \ldots$. . . . . . . . . . . . . . 14

2.5 Schematic illustration of the SURF descriptor computation adopted from [BETG08]. 16

2.6 Descriptor entries of a SURF descriptor sub-region for three different image intensity distributions. Image reproduced from [BETG08]. . . . . . . . . . . 16

2.7 Example of a kd-tree structure in a two dimensional space. Each plane, indicated by their respective number, separates the set of data points, indicated by a letter, into two approximately equally sized subsets. After a few subdivisions, every leave node consists of only one data point each (in this example). A nearest neighbor (NN) to a new data point is found by traversing the tree $\begin{array}{ll}\text { structure to identify the corresponding leave and thus the NN candidate(s). . } & 18\end{array}$

2.8 Iteration steps of the k-means algorithm. $K=3$ centroids (red stars) are trained to represent the blue data points. The initial step is shown in (a) where random points are selected as centroids. All blue data points are assigned to their closest centroid forming three sets. The means of these three sets define the new centroids shown in (b). This process continues until the positions of the centroids converge (e). Red lines indicate equal distances to the closest centroids and are the borders of the so-called Voronoi cells defining the area covered by a centroid. . . . . . . . . . . . . . . . 19 
2.9 Summary of the Bag-of-Feature vector computation. Interest points / regions are detected in an image and the corresponding texture is robustly summarized in a feature descriptor vector (see Section 2.2.1 for details). Descriptor vectors are quantized into representatives termed visual words via, e.g., k-means clustering. Ultimately the BoF vector is formed as a visual word frequency histogram where the entries are weighted in accordance to their Inverse Document

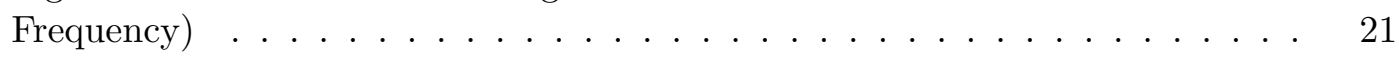

2.10 Illustration of a tree based BoF quantization and indexing structure. Image patches assigned to a single node decrease in diversity with increasing descriptor quantization. The leaf nodes are defined as visual words with associated inverted files. These reference the images to be scored when a query image patch is quantized to the respective visual word. (Reproduced from $\left[\mathrm{SHC}^{+} 11\right]$,

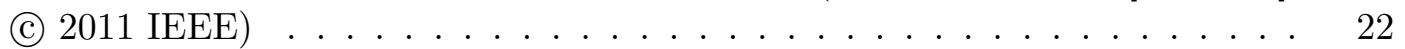

2.11 Illustration of a BoF-based image retrieval system. The gray modules have to be performed only once to setup (train) the system. . . . . . . . . . . 23

2.12 Schematic illustration of the individual training stages of the hierarchical kmeans quantizer (HKM). Colored dots are trained centroids, the edges indicate the borders of the corresponding Voronoi cells. Image reproduced from [NS06]. 25

2.13 Comparison of state-of-the-art quantization and indexing structures based on a Google Street View database. Panoramas within a radius of $10 \mathrm{~m}$ around the query location are considered relevant. (Reproduced from [SHC $\left.{ }^{+} 11\right]$, (c) 2011

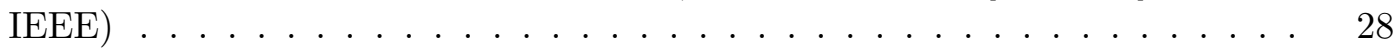

2.14 Evaluation of soft assignment $\mathrm{PCI}^{+} 08$ applied to AKM and HKM quantization structures. Panoramas within a radius of $20 \mathrm{~m}$ around the query location are considered relevant. (Reproduced from $\left[\mathrm{SHC}^{+} 11\right]$, (c) 2011 IEEE) $\ldots$. . . . 29

3.1 Google Street View panorama matched to a low resolution video recording (downtown Pittsburgh) using the Multiple Hypothesis Vocabulary Tree. The red ellipse indicates the parts common to both query frame and retrieved panorama. (Reproduced from $\left[\mathrm{SANH}^{+} 11\right]$, (c) 2011 IEEE) $] \ldots \ldots$. . . . . . . . 33

3.2 Sample images from the Google Street View dataset of Pittsburgh; panoramas are on average $12.6 \mathrm{~m}$ apart from each other. (Reproduced from $\left[\mathrm{SANH}^{+} 11\right]$,

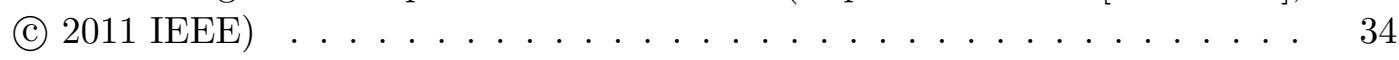

3.3 Comparison of state-of-the-art quantization and indexing structures based on a Google Street View database. Panoramas within a radius of $10 \mathrm{~m}$ around the query location are considered relevant. (Reproduced from $\left[\mathrm{SANH}^{+} 11\right]$, (c)

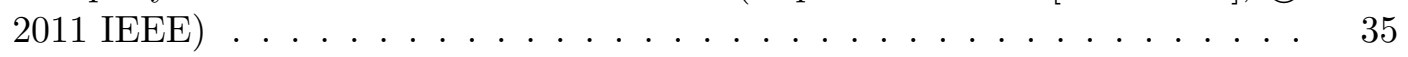

$3.4 \quad$ Quantization with overlapping buffers. (Reproduced from [SANH $\left.{ }^{+} 11\right]$, (c) 2011

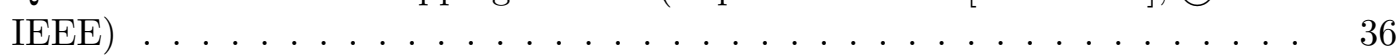

3.5 Two dimensional illustration of the separation axis $\tilde{\mathbf{u}}$, the overlapping buffer, and the determination of the false quantization probability $P_{f}(d)$ as a function of the distance $d$ between the query feature and the separating hyper plane. (Reproduced from $\left[\mathrm{SANH}^{+} 11\right]$, (c) 2011 IEEE) $\ldots \ldots \ldots$. . . . . . . . . . 37

3.6 Comparison of MHVT with weighted scoring and HKM at two vicinity levels. (Reproduced from $\left[\mathrm{SANH}^{+} 11\right]$, (c) $\left.\left.2011 \mathrm{IEEE}\right)\right] \ldots \ldots \ldots \ldots$. . . . . . . 
4.1 Gnomonic projections of a Google Street View Panorama recorded in San Francisco. Ellipses are fitted around affine covariant MSER [MCUP04] regions. Features attached to foliage are successfully detected and removed. (Reproduced from $\left[\mathrm{SHC}^{+} 11\right]$, (c) 2011 IEEE) $] \ldots \ldots \ldots \ldots$. . . . . . . . . . . . . . . 42

4.2 Spatial partitioning of the search space in four regions / trees, which overlap by $50 \%$ in horizontal and vertical direction; The double shaded area in the middle is covered by four trees. (Reproduced from $\left[\mathrm{SHC}^{+} 11\right]$, (c) 2011 IEEE)]

4.3 Evaluation of retrieval performance for realistic query video recordings. The number of retrieved locations within a given radius around the true query location is plotted against the frame number. (Reproduced from $\left[\mathrm{SHC}^{+} 11\right]$, (c)

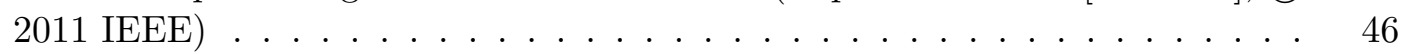

5.1 Overview of the visual location recognition system architecture of Chapter 3 , (Reproduced from $\left[\mathrm{SHC}^{+} 11\right]$, (c) 2011 IEEE) $] \ldots \ldots \ldots \ldots \ldots$

5.2 Overview of a visual location recognition system where the retrieval task is shifted from the server to the client by transferring the quantization structure and compressed inverted file system of the most suitable subregion. Dashed lines indicate the data transmission during initialization. Once the transfer of the quantization structure and the inverted file is finished, no further network delay is introduced. (Reproduced from $\left[\mathrm{SHC}^{+} 11\right]$, (c) 2011 IEEE) $] \ldots . . .248$

5.3 Overview of a visual location recognition system where relevant parts of the vocabulary are periodically pushed to the client. The selection of the transmitted visual words is based on the location retrieval performed at the server using the query features uploaded from the client. The pose estimation is performed on the mobile device utilizing these preloaded adaptive partial vocabularies. Blue lines indicate that the network delay does not influence the time required for the location recognition. (Reproduced from [SHC+11$]$, (c) 2011 IEEE)] . .

5.4 Quantization using partial vocabularies. Limiting the quantization to a subset (green dots) of the full vocabulary results in enlarged Voronoi cells that fully contain the cells of the full vocabulary (yellow areas). $\ldots \ldots \ldots \ldots$. . . .

5.5 Evaluation of the location recognition performance of adaptive partial vocabularies using the same dataset and metrics as in Figure 4.3 . A significant increase in the number of correctly retrieved panoramas per query location can

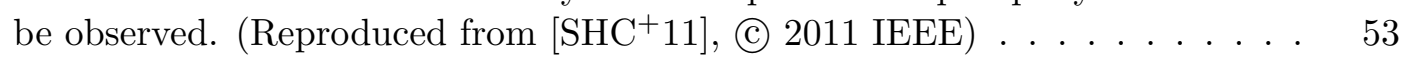

5.6 mAP scores for multiple partial vocabulary configurations over the update rate

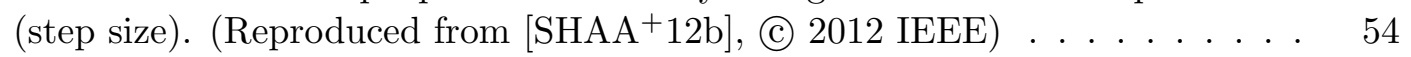

5.7 mAP score of the K1R40 partial vocabulary configuration at three different data rates over the update rate (step size). (Reproduced from $\left[\mathrm{SHAA}^{+} 12 \mathrm{~b}\right]$,

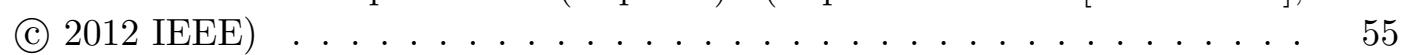

5.8 Number of new visual words sent to the client per update (key frame) at two different data rates and a step size of 100 frames. (Reproduced from $\left[\mathrm{SHAA}^{+} 12 \mathrm{~b}\right]$,

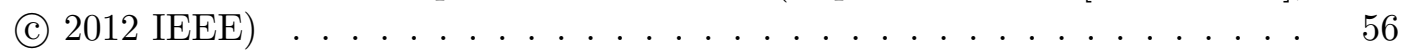

5.9 Change in mAP for the K1R40 configuration at a data rate of $0.25 \mathrm{Mbit} / \mathrm{s}$ w.r.t. using the full vocabulary at individual frames. (Reproduced from [SHAA $\left.{ }^{+} 12 \mathrm{~b}\right]$,

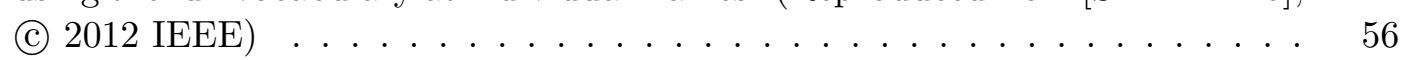


5.10 Change in mAP using particle filter based partial vocabularies at $0.25 \mathrm{Mbit} / \mathrm{s}$ with respect to using the full vocabulary at individual frames. (Reproduced

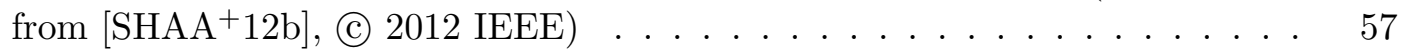

6.1 Exploiting not only the information given by the text but also its unique appearance by describing characters as visual words allows us to differentiate the two cafe signs. (Reproduced from $\left[\mathrm{SHH}^{+} 11\right]$, (c) 2011 IEEE) $]$. . . . . . . . . 60

6.2 Extracting text from a natural image. (a): Detected MSER for dark objects on bright background. (b): After geometric and stroke width filtering, text candidates are pairwise grouped to form text lines. The text lines are shown by the red lines. (c): Text line verification rejects false positives and the detected text is highlighted by the blue box. (Reproduced from $\left[\mathrm{CTS}^{+} 11\right]$, (c)

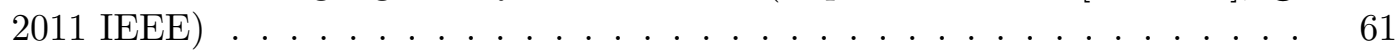

6.3 System flowchart. (Reproduced from $\left[\mathrm{CTS}^{+} 11\right]$, (c) 2011 IEEE) . . . . . . . . 62

6.4 Edge-enhanced MSER. (a) Detected MSER for blurred text. Canny edges are shown in red lines and the blue arrows indicate gradient directions. (b) MSER after pruning along the gradient. (Reproduced from $\left[\mathrm{CTS}^{+}\right.$11], (c) 2011 IEEE) 64

6.5 Finding the stroke width information. (a) Mesh plot of the distance transformed image. (b) Stroke width image formed by propagating the stroke width information from the ridge to the boundary. The numbers label half of the stroke width since the ridge values correspond to half of the actual width. (Reproduced from $\left[\mathrm{CTS}^{+} 11\right]$, (c) 2011 IEEE) $\ldots \ldots \ldots$. . . . . . . . . . 64

6.6 Word separation via binary letter distance classification. (a) The text line is separated into words, based on the vertical projection distances. (b) The distribution of the spacing is classified into two regions using Otsu's method. (Reproduced from $\left[\mathrm{CTS}^{+} 11\right]$, (c) 2011 IEEE) $\ldots \ldots \ldots \ldots$. . . . . . . . 66

6.7 Document images under various view points. (Reproduced from [TCC $\left.{ }^{+} 11\right]$, (C)

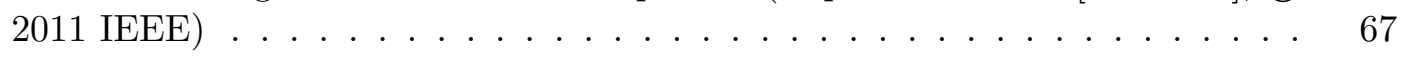

6.8 Text detection using the EMSER approach described in Section 6.1. Detected character regions are shown in red color. (Reproduced from [SHH $\left.{ }^{+} 11\right]$, (C) 2011

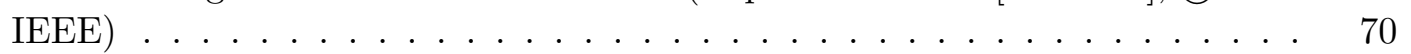

6.9 Schematic illustration of the text based image retrieval approach. Characters are detected via EMSER and represented using SURF descriptors. A quantization of the descriptors into visual words allows us to assign a visual word ID to each detected character. Strings of characters are represented by subsequences (N-grams) of successive visual word IDs. Image retrieval is performed by identifying those images that include the same N-grams using an inverted file structure. (Reproduced from $\left[\mathrm{SHH}^{+} 11\right]$, (c) 2011 IEEE) $] \ldots$. . . . . . . . 71

6.10 Image recordings in Munich using a camcorder. Despite the presence of the dynamic objects, the complex 3-dimensional scene and the large baselines, the images a-c are reliably matched using the proposed text based image retrieval engine. Detected letters are highlighted by yellow ellipses. (Reproduced from $\left[\mathrm{SHH}^{+} 11\right]$, (c) 2011 IEEE) $] \ldots \ldots \ldots \ldots$. . . . . . . . . . . . . . . 72

6.11 Visual Word Samples for $S=10$. . . . . . . . . . . . . . . . 74

6.12 Visual Word Samples for $S=100 \ldots \ldots \ldots$. . . . . . . . . . 75

6.13 Visual Word Samples for $S=1000 \ldots \ldots \ldots \ldots$. . . . . . . . . 75 
6.14 Relative N-gram occurrence frequencies in the dataset recorded in downtown

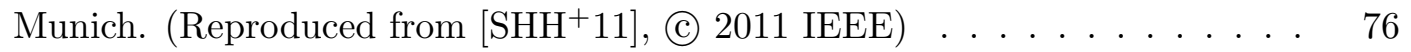

6.15 Exemplary plot of the mutual information against vocabulary size $S$ and N-

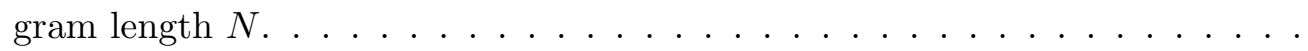

6.16 Example Images of Pittsburgh Street View Dataset . . . . . . . . . . . . . . 81

$6.17 \mathrm{mAP}$ score of selected N-gram configurations at varying vocabulary size $S$ using the Pittsburgh Street View dataset. (Reproduced from [SHH ${ }^{+}$11], (c) 2011 IEEE) 82

$6.18 \mathrm{mAP}$ score of selected N-gram configurations at vocabulary size 400 and varying radius defining the relevant images in the Pittsburgh Street View dataset.

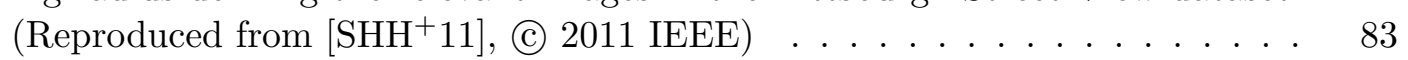

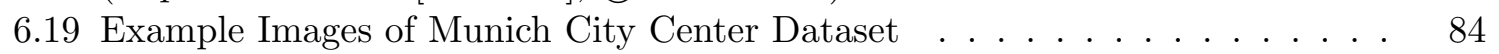

$6.20 \mathrm{mAP}$ score of selected N-gram configurations at varying vocabulary size $S$ using

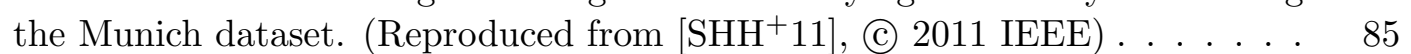

$6.21 \mathrm{mAP}$ score of single N-grams at varying vocabulary size $S$ using the Amazon

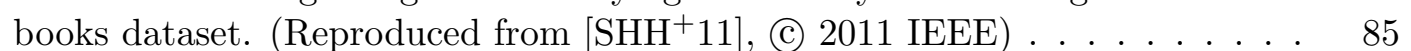

6.22 Example Images of Amazon Book Covers Dataset . . . . . . . . . . . . . . 86

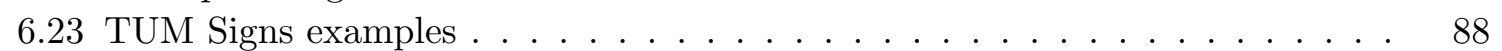

6.24 TUM Signs query examples $\ldots \ldots \ldots$. . . . . . . . . . . . . . . . . . 89

$6.25 \mathrm{mAP}$ for TUM Signs normal dataset BoF $\ldots \ldots \ldots \ldots$. . . . . . . . 89

$6.26 \mathrm{mAP}$ for TUM Signs normal dataset text-related . . . . . . . . . . . . . . 90

6.27 TUM Signs text-related and BoF Features . . . . . . . . . . . . . . . . . . . 91

$6.28 \mathrm{mAP}$ for TUM Signs fixed panned dataset text-related . . . . . . . . . . . . . 91

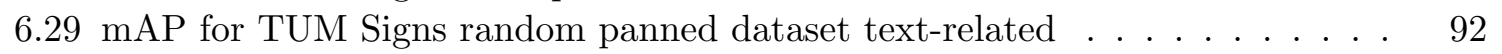

7.1 Point cloud acquired by registering scans from the vertically mounted laser scanner. By casting rays from camera positions into the point cloud, world coordinates of local image features are determined. (Reproduced from $\left[\mathrm{HSH}^{+} 12 \mathrm{~b}\right]$,

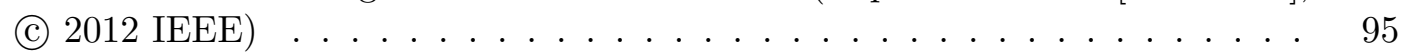

7.2 The mapping trolley used for dataset acquisition. (Reproduced from $\left[\mathrm{HSH}^{+} 12 \mathrm{a}\right]$,

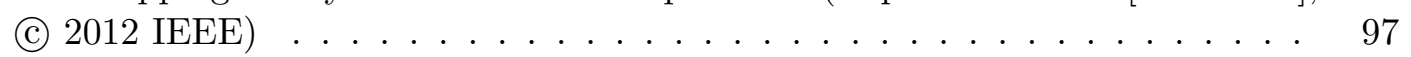

7.3 The eight images captured by panoramic camera and the two DSLRs every

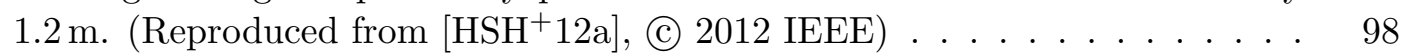

7.4 Maps built with data from horizontally mounted laser scanner. The total distance traveled during acquisition is $4.5 \mathrm{~km}$; the length of mapped corridors is about $2.3 \mathrm{~km}$. (Reproduced from $\left[\mathrm{HSH}^{+} 12 \mathrm{a} \mid\right.$, (c) 2012 IEEE) $\ldots . . . . .999$

7.5 Sample images and frame numbers from query video snippets. . . . . . . . . . 100

7.6 Locations of the generated virtual views (blue dots), the query frames (red crosses), as well as the radius around the query location defining the relevant virtual view points. A radius of $5 \mathrm{~m}$ is applied. . . . . . . . . . . . . 101

7.7 Illustration of the camera setup for the upcoming mapping platform. . . . . . 102

7.8 Images captured during mapping (blue dots, heading in red) and the virtual viewpoints created (grey dots). At each location, 16 views are computed (red compass rose). (Reproduced from $\left[\mathrm{HSH}^{+} 12 \mathrm{~b}\right]$, (c) 2012 IEEE) $\mid \ldots \ldots$. . . . . . 104

7.9 The point cloud acquired during mapping is segmented into planes (indicated by different colors) that provide the models for projective transforms. (Reproduced from $\left[\mathrm{HSH}^{+} 12 \mathrm{~b}\right]$, (c) 2012 IEEE) $] \ldots \ldots \ldots \ldots$. . . . . . . . . . . . . . . . . . . 105

7.10 TUM Signs text-related and BoF Features . . . . . . . . . . . . . 106 
7.11 Intensity comparison pattern of BRIEF $\ldots \ldots \ldots \ldots$. . . . . . . 108

7.12 LSH timing, precision and percentage of Mmtched features . . . . . . . . . . 110

7.13 Top-ranked retrieval results for 4 query images (black square is the ground truth pose). Location and orientation are drawn as circles (rank 1: red, 2: green, 3: blue, 4: orange). No post-processing of image retrieval results has been applied. (Reproduced from $\left[\mathrm{HSH}^{+}\right.$12b], (c) 2012 IEEE) $] \ldots \ldots$. . . . . . . 111 


\section{List of Tables}

$6.1 \quad$ Evaluation of text detection algorithms. . . . . . . . . . . . . . 67

7.1 Overview of mapping runs. Two 18 Megapixel digital single-lens reflex images and six 2 Megapixel Ladybug images are captured at each location and are tagged with the corresponding 6-DOF pose. . . . . . . . . . . . . . 101

7.2 Mean precision at cutoff ranks 1,3 and 5. Relevant views are within radius $r$ around the query location. . . . . . . . . . . . . . . . 112

7.3 Comparison of SIFT and BRIEF feature based location recognition. Mean precision scores are provided at cutoff ranks 1,3 and 5. Relevant views are within $5 \mathrm{~m}$ around the query location. . . . . . . . . . . . . . . . . 114 


\section{Bibliography}

\section{Publications by the author}

[CTS $\left.{ }^{+} 11\right] \quad$ H. Chen, S. Tsai, G. Schroth, D. Chen, R. Grzeszczuk, and Bernd Girod. Robust Text Detection in Natural Images with Edge-Enhanced Maximally Stable Extremal Regions. In International Conference on Image Processing, Brussels, September 2011.

$\left[\mathrm{HMH}^{+} 12\right] \quad$ S. Hilsenbeck, A. Möller, R. Huitl, G. Schroth, M. Kranz, and E. Steinbach. Scale-preserving long-term visual odometry for indoor navigation. In International Conference on Indoor Positioning and Indoor Navigation (IPIN), Sydney, Australia, 2012.

[HSH $\left.{ }^{+} 12 \mathrm{a}\right] \quad$ R. Huitl, G. Schroth, S. Hilsenbeck, F. Schweiger, and E. Steinbach. TUMindoor: an extensive image and point cloud dataset for visual indoor localization and mapping. In International Conference on Image Processing, 2012.

$\left[\mathrm{HSH}^{+} 12 \mathrm{~b}\right] \quad$ R. Huitl, G. Schroth, S. Hilsenbeck, F. Schweiger, and E. Steinbach. Virtual reference view generation for cbir-based visual pose estimation. In ACM Multimedia, short paper, Nara, Japan, 2012.

[NK09] Patrick Nepper and Nikolaus Konrad, editors. The Future of Social Commerce - Trend Report 2007/2008. CDTM, 2009. ISBN 978-3-9812203-1-5.

[RSBM09] M. Rippl, G. Schroth, B. Belabbas, and M. Meurer. A probabilistic assessment on the Range Consensus (RANCO) RAIM Algorithm. In ION International Technical Meeting (ITM), Anaheim, California, January 2009.

$\left[\mathrm{SANH}^{+} 11\right]$ G. Schroth, A. Al-Nuaimi, R. Huitl, F. Schweiger, and E. Steinbach. Rapid Image Retrieval for Mobile Location Recognition. In International Conference on Acoustics, Speech and Signal Processing, Prague, May 2011.

[Sch11] G. Schroth. Method of operating a satellite navigation receiver, 2011. Patent: US20090273511A1, EP000002120060B1, AT000000474233E.

[Sch13] Georg Schroth. Mobile Visual Location Recognition. PhD thesis, Technische Universität München, July 2013.

[SCSG11] G. Schroth, N. Cheung, E. Steinbach, and B. Girod. Synchronization of presentation slides and lecture videos using bit rate sequences. In International Conference on Image Processing, Brussels, September 2011.

$\left[\mathrm{SEB}^{+} 08\right] \quad$ G. Schroth, A. Ene, J. Blanch, T. Walter, and P. Enge. Failure Detection and Exclusion via Range Consensus. In European Navigation Conference GNSS, Toulouse, France, April 2008.

$\left[\mathrm{SES}^{+} 12\right] \quad$ F. Schweiger, M. Eichhorn, G. Schroth, E. Steinbach, and M. Fahrmair. Method and an apparatus for performing a cross-calculation, 2012. Patent: US20120155777. 
[SgWD09] G. Schroth, I. Stork genannt Wersborg, and K. Diepold. A cognitive system for autonomous robotic welding. In International Conference on Intelligent Robots and Systems, IROS, St. Louis, Missouri, October 2009.

[SHAA ${ }^{+}$12a] G. Schroth, R. Huitl, M. Abu-Alqumsan, A. Al-Nuaimi, F. Schweiger, and E. Steinbach. Visual Localization Method, 2012. Patent: PCTEP2011057901.

$\left[\mathrm{SHAA}^{+} 12 \mathrm{~b}\right]$ G. Schroth, R. Huitl, M. Abu-Alqumsan, F. Schweiger, and E. Steinbach. Exploiting prior knowledge in mobile visual location recognition. International Conference on Acoustics, Speech and Signal Processing, 2012.

$\left[\mathrm{SHC}^{+} 11\right] \quad$ G. Schroth, R. Huitl, D. Chen, M. Abu-Alqumsan, A. Al-Nuaimi, and E. Steinbach. Mobile Visual Location Recognition. In IEEE Signal Processing Magazine; Special Issue on Mobile Media Search, volume 28, pages 77-89, July 2011.

$\left[\mathrm{SHH}^{+} 11\right] \quad$ G. Schroth, S. Hilsenbeck, R. Huitl, F. Schweiger, and E. Steinbach. Exploiting text-related features for content-based image retrieval. In International Symposium on Multimedia (ISM), Dana Point, CA, USA, December 2011.

$\left[\mathrm{SHH}^{+}{ }^{12}\right] \quad$ G. Schroth, S. Hilsenbeck, R. Huitl, F. Schweiger, and E. Steinbach. Verfahren zur Suche von visuell ähnlichen Bildern in einer Sammlung von Referenzbildern, 2012. Patent: DE102011119941.5.

[SPH07] G. Schroth, C. Preusche, and P. Hinterseer. Astronautic Communication for Telepresence Applications. In International Conference on Robotics and Applications and Telematics, Würzburg, Germany, August 2007.

[SRBM08] G. Schroth, M. Rippl, B. Belabbas, and M. Meurer. Enhancements of the Range Consensus Algorithm (RANCO). In 4th ESA Workshop on Satellite Navigation User Equipment Technologies, Noordwijk, The Netherlands, December 2008.

$\left[\mathrm{SRE}^{+} 08\right] \quad$ G. Schroth, M. Rippl, A. Ene, J. Blanch, B. Belabbas, T. Walter, and P. Engeand M. Meurer. Enhancements of the Range Consensus Algorithm (RANCO). In Institute of Navigation, Savannah, GA, USA, September 2008.

[SSE $\left.{ }^{+} 10 \mathrm{a}\right]$ G. Schroth, F. Schweiger, M. Eichhorn, E. Steinbach, M. Fahrmair, and W. Kellerer. Method and apparatus for synchronizing video data, 2010. Patent: US020110122315A1, EP000002326091A1, JP002011109656A.

[SSE $\left.{ }^{+} 10 \mathrm{~b}\right] \quad$ G. Schroth, F. Schweiger, M. Eichhorn, E. Steinbach, M. Fahrmair, and W. Kellerer. Video Synchronization using Bit Rate Profiles. In International Conference on Image Processing (ICIP), Hong Kong, September 2010.

$\left[\mathrm{SSE}^{+} 11\right] \quad$ F. Schweiger, G. Schroth, M. Eichhorn, E. Steinbach, and M. Fahrmair. Consensus-based Cross-correlation. In ACM Multimedia, short paper, Scottsdale, AZ, November 2011.

[SSE $\left.{ }^{+} 12\right] \quad$ F. Schweiger, G. Schroth, M. Eichhorn, A. Al-Nuaimi, B. Cizmeci, M. Fahrmair, and E. Steinbach. Fully automatic and frame-accurate video synchronization using bitrate sequences. IEEE Transactions in Multimedia, 2012.

$\left[\mathrm{SZG}^{+} 09\right] \quad$ F. Schweiger, B. Zeisl, P. Georgel, G. Schroth, E. Steinbach, and N. Navab. Maximum Detector Response Markers for SIFT and SURF. In Vision, Modeling and Visualization Workshop (VMV), Braunschweig, November 2009.

$\left[\mathrm{TCC}^{+} 11\right] \quad$ S. S. Tsai, H. Chen, D. M. Chen, G. Schroth, R. Grzeszczuk, and B. Girod. Mobile visual search on papers using text and low bit-rate features. In International Conference on Image Processing, Brussels, September 2011. 


\section{General publications}

[AA10] M. Abu-Alqumsan. Low latency mobile vision-based localization. Master's thesis, Technische Universität München, 2010. Supervisor: Georg Schroth.

[AFDM08] A. Angeli, D. Filliat, S. Doncieux, and J.A. Meyer. A fast and incremental method for loop-closure detection using bags of visual words. IEEE Trans. on Robotics, Special Issue on Visual SLAM, 24:1027-1037, 2008.

[Ama12] Amazon SnapTell. http://a9.com/-/company/visualsearch.jsp, Last accessed in 2012 .

[AN09] A. Al-Nuaimi. Rapid feature matching. Master's thesis, Technische Universität München, 2009. Supervisor: Georg Schroth.

[AOV12] A. Alahi, R. Ortiz, and P. Vandergheynst. FREAK: Fast retina keypoint. In IEEE Conference on Computer Vision and Pattern Recognition (CVPR) (To Appear), Providence, Rhode Island, 2012. IEEE.

[Bai04] D.G. Bailey. An efficient euclidean distance transform. In Combinatorial Image Analysis, IWCIA, pages 394-408, 2004.

[BETG08] H. Bay, A. Ess, T. Tuytelaars, and L.V. Gool. Speeded-up robust features (surf). Computer Vision and Image Understanding, 110(3):346 - 359, 2008.

[BK03] S. Burkhardt and J. Kärkkäinen. Better Filtering with Gapped-q-Grams. Fundamenta Informaticae, 23:1001-1018, 2003.

[BL97] Jeffrey S. Beis and David G. Lowe. Shape Indexing Using Approximate NearestNeighbour Search in High-Dimensional Spaces. In IEEE Int. Conf. Comp. Vision Pattern Recognition, San Juan, August 1997.

[Can86] J. Canny. A computational approach to edge detection. IEEE Trans. Pattern Anal. Mach. Intell., 8:679-698, 1986.

$\left[\mathrm{CBK}^{+} 11\right]$ David Chen, Georges Baatz, Kevin Köser, Sam Tsai, Ramakrishna Vedantham, Timo Pylvänäinen, Kimmo Roimela, Xin Chen, Jeff Bach, Marc Pollefeys, Bernd Girod, and Radek Grzeszczuk. City-scale landmark identification on mobile devices. In IEEE Int. Conf. Comp. Vision Pattern Recognition, Colorado Springs, CO, June 2011.

[CDM08] J. Civera, A.J. Davison, and J. Montiel. Inverse depth parametrization for monocular SLAM. Robotics, IEEE Transactions on, 24(5):932-945, 2008.

[CLSF10] M. Calonder, V. Lepetit, C. Strecha, and P. Fua. BRIEF: Binary robust independent elementary features. European Conference on Computer Vision (ECCV), pages $778-792,2010$.

[CN07] S. Chaudhuri and V.P. Namboodiri. Retrieval of images of man-made structures based on projective invariance. Pattern Recognition, 40(1):296-308, 2007.

[CP06] S.Y. Cho and C.G. Park. MEMS based pedestrian navigation system. Journal of Navigation, 59(01):135-153, 2006.

$\left[\mathrm{CPS}^{+} 07\right]$ Ondrej Chum, James Philbin, Josef Sivic, Michael Isard, and Andrew Zisserman. Total Recall: Automatic Query Expansion with a Generative Feature Model for Object Retrieval. In Int. Conf. on Comp. Vision, Rio de Janeiro, October 2007.

[CTC ${ }^{+}$09a] V. Chandrasekhar, G. Takacs, D. Chen, S. Tsai, R. Grzeszczuk, and B. Girod. 
Chog: Compressed histogram of gradients. a low bit-rate feature descriptor. In CVPR, pages $2504-2511,2009$.

$\left[\mathrm{CTC}^{+}\right.$09b] D.M. Chen, S.S. Tsai, V. Chandrasekhar, G. Takacs, J. Singh, and B. Girod. Tree histogram coding for mobile image matching. In IEEE Data Compression Conference, Snowbird, March 2009.

[CTS $\left.{ }^{+} 11\right] \quad H$. Chen, S. Tsai, G. Schroth, D. Chen, R. Grzeszczuk, and Bernd Girod. Robust Text Detection in Natural Images with Edge-Enhanced Maximally Stable Extremal Regions. In International Conference on Image Processing, Brussels, September 2011.

$\left[\mathrm{CWL}^{+} 10\right]$ Y. Cao, C. Wang, Z. Li, L. Zhang, and L. Zhang. Spatial-Bag-of-Features. In IEEE Int. Conf. on Comp. Vision and Pattern Recognition, San Francisco, June 2010.

[CY04] X. Chen and A.L. Yuille. Detecting and reading text in natural scenes. In CVPR, volume 2, pages II-366 - II-373 Vol.2, 2004.

[CY05] X. Chen and A.L. Yuille. A time-efficient cascade for real-time object detection: With applications for the visually impaired. In CVPR - Workshops, page 28, 2005.

[Dam64] Fred J Damerau. A technique for computer detection and correction of spelling errors. Communications of the ACM, 7(3):171-176, 1964.

[Dav03] A.J. Davison. Real-time simultaneous localisation and mapping with a single camera. In ICCV, pages 1403-1410, 2003.

[DB06] M. Donoser and H. Bischof. Efficient maximally stable extremal region (MSER) tracking. In IEEE Int. Conf. on Comp. Vision and Pattern Recognition, pages 553 - 560, New York, June 2006.

[DIIM04] Mayur Datar, Nicole Immorlica, Piotr Indyk, and Vahab S. Mirrokni. Localitysensitive hashing scheme based on p-stable distributions. In Symp. on Computational Geometry, New York, June 2004.

[DJLW08] R. Datta, D. Joshi, J. Li, and J.Z. Wang. Image retrieval: Ideas, influences, and trends of the new age. ACM Computing Surveys (CSUR), 40(2):1-60, 2008.

[DRMS07] A.J. Davison, I. Reid, N. D. Molton, and O. Stasse. MonoSLAM: Real-time single camera SLAM. IEEE Trans. Pattern Analysis and Machine Intelligence, 29(6):1052-1067, June 2007.

[EOW10] B. Epshtein, E. Ofek, and Y. Wexler. Detecting text in natural scenes with stroke width transform. In CVPR, pages $2963-2970,2010$.

[FB81] M.A. Fischler and R.C. Bolles. Random sample consensus: A paradigm for model fitting with applications to image analysis and automated cartography. Communications of the ACM, 24(6):381-395, 1981.

[FBF77] Jerome H. Friedman, Jon Louis Bentley, and Raphael Ari Finkel. An Algorithm for Finding Best Matches in Logarithmic Expected Time. ACM Trans. Mathematical Software, 3(3):209-226, September 1977.

[FCM09] J. Fabrizio, M. Cord, and B. Marcotegui. Text extraction from street level images. pages 199-204, 2009.

$\left[\mathrm{GCC}^{+} 11\right]$ B. Girod, V. Chandrasekhar, D. M. Chen, N. M. Cheung, R. Grzeszczuk, 
Y. Reznik, G. Takacs, S. S. Tsai, and R. Vedantham. Mobile Visual Search. In IEEE Signal Processing Magazine; Special Issue on Mobile Media Search, volume 28, pages 61-76, July 2011.

[GEW06] P. Geurts, D. Ernst, and L. Wehenkel. Extremely randomized trees. Machine Learning, 63(1):3-42, 2006.

[Goo12b] Google Glass Project. https://plus.google.com/+projectglass/posts, Last accessed in 2012.

[Goo12c] Google Goggles. http://www.google.de/mobile/goggles/, Last accessed in 2012.

[Goo12d] Google Street View. http://maps.google.com/streetview, Last accessed in 2012.

[GSB07] G. Grisetti, C. Stachniss, and W. Burgard. Improved techniques for grid mapping with rao-blackwellized particle filters. Robotics, IEEE Transactions on, 23(1):3446, 2007.

[Ham50] Richard W Hamming. Error detecting and error correcting codes. Bell System Technical Journal, 29:147-160, 1950.

[Hil11] S. Hilsenbeck. Exploiting text-related features for content-based image retrieval. Master's thesis, Technische Universität München, 2011. Supervisor: Georg Schroth.

[Hui10] R. Huitl. Fast image retrieval for mobile location recognition. Master's thesis, Technische Universität München, 2010. Supervisor: Georg Schroth.

$\left[\mathrm{JDC}^{+} 11\right]$ Rongrong Ji, Ling-Yu Duan, Jie Chen, Hongxun Yao, and Wen Gao. A lowbit rate vocabulary coding scheme for mobile landmark search. In Intl. Conf. on Acoustics, Speech and Signal Proc., Prague, 2011.

[JDS10] H. Jégou, M. Douze, and C. Schmid. Improving bag-of-features for large scale image search. Int. Journal of Comp. Vision, 87(3):316-336, February 2010.

[JDSP10] Hervé Jégou, Matthijs Douze, Cordelia Schmid, and Patrick Pérez. Aggregating local descriptors into a compact image representation. In IEEE Conf. on Comp. Vision 83 Pattern Recognition, San Francisco, June 2010.

[JEGE04] Joseph O'Rourke Jacob E. Goodman and Piotr Indyk (Ed.). Handbook of Discrete and Computational Geometry. CRC Press, 2nd edition, 2004.

[JKJ04] K. Jung, K.I. Kim, and A.K. Jain. Text information extraction in images and video: a survey. Pattern Recognition, 37(5):977 - 997, 2004.

[JL06] W.Y. Jeong and K.M. Lee. Visual slam with line and corner features. In Intelligent Robots and Systems, 2006 IEEE/RSJ International Conference on, pages 2570-2575. IEEE, 2006.

[KA08] Kurt Konolige and Motilal Agrawal. FrameSLAM: From bundle adjustment to real-time visual mapping. IEEE Transactions on Robotics, 24(5):1066-1077, 2008.

[KCS11] L. Kneip, M. Chli, and R. Siegwart. Robust real-time visual odometry with a single camera and an imu. measurements, 23(12):1157, 2011.

[KM07] Georg Klein and David W. Murray. Parallel tracking and mapping for small AR workspaces. In ISMAR, pages 225-234. IEEE, 2007.

[KM08] Georg Klein and David Murray. Improving the agility of keyframe-based SLAM. In $E C C V$, pages II: 802-815, 2008. 
[KRD08] M. Kaess, Ananth Ranganathan, and F. Dellaert. iSAM: Incremental smoothing and mapping. IEEE Transactions on Robotics, 24(6):1365-1378, 2008.

[KS11] J. Kelly and G. S. Sukhatme. Visual-inertial sensor fusion: Localization, mapping and sensor-to-sensor self-calibration. The Int'l Journal of Robotics Research, 30(1):56-79, 2011.

[KWS11] L. Kneip, S. Weiss, and R. Siegwart. Deterministic initialization of metric state estimation filters for loosely-coupled monocular vision-inertial systems. In 2011 IEEE/RSJ Int'l Conference on Intelligent Robots and Systems (IROS), pages 2235-2241, September 2011.

$\left[\mathrm{LCC}^{+} 10\right]$ T. Liu, M. Carlberg, G. Chen, J. Chen, J. Kua, and A. Zakhor. Indoor localization and visualization using a human-operated backpack system. In Proc. Intl. Conf. on Indoor Positioning and Indoor Navigation (IPIN), Zürich, Switzerland, pages 1-10, 2010.

[LCS11] S. Leutenegger, M. Chli, and R.Y. Siegwart. BRISK: Binary robust invariant scalable keypoints. In IEEE International Conference on Computer Vision (ICCV), pages 2548-2555, Barcelona, Spain, 2011. IEEE.

[LDBL07] H. Liu, H. Darabi, P. Banerjee, and J. Liu. Survey of wireless indoor positioning techniques and systems. IEEE Transactions on Systems, Man, and Cybernetics, Part C: Applications and Reviews, 37(6):1067-1080, 2007.

[LDL05] J. Liang, D. Doermann, and H.P. Li. Camera-based analysis of text and documents: a survey. 7(2-3):84-104, 2005.

[Lev66] Vladimir Levenshtein. Binary codes capable of correcting deletions, insertions, and reversals. Soviet Physics Doklady, 10(8):707-710, 1966.

[LFWL09] J. Li, Z.G. Fan, Y. Wu, and N. Le. Document image retrieval with local feature sequences. In Int. Conf. on Document Analysis and Recognition, Barcelona, July 2009 .

[LLT08] Shijian Lu, Linlin Li, and Chew Lim Tan. Document Image Retrieval through Word Shape Coding. IEEE Transactions on Pattern Analysis and Machine Intelligence, 30:1913-1918, 2008.

[LMGY04] T. Liu, A.W. Moore, A. Gray, and K. Yang. An investigation of practical approximate nearest neighbor algorithms. In Neural Information Processing Systems, Vancouver, May 2004.

[Low04] D.G. Lowe. Distinctive image features from scale-invariant keypoints. International Journal of Computer Vision, 60:91-110, 2004.

[LPS ${ }^{+}$03] S.M. Lucas, A. Panaretos, L. Sosa, A. Tang, S. Wong, and R Young. Icdar 2003 robust reading competitions. In ICDAR, volume 2, page 682, 2003.

[LSP06] S. Lazebnik, C. Schmid, and J. Ponce. Beyond bags of features: Spatial pyramid matching for recognizing natural scene categories. In IEEE Int. Conf. Comp. Vision Pattern Recognition, New York, June 2006.

[Luc05] S.M. Lucas. Icdar 2005 text locating competition results. In ICDAR, pages $80-$ 84 Vol. 1, 2005.

[LW02] Rainer Lienhart and Axel Wernicke. Localizing and segmenting text in images 
and videos. Circuits and Systems for Video Technology, IEEE Transactions on, 12(4):256-268, 2002.

$\left[\mathrm{LYK}^{+}\right.$09] Q. Liu, H. Yano, D. Kimber, C. Liao, and L. Wilcox. High accuracy and language independent document retrieval with a fast invariant transform. In Int. Conf. on Multimedia and Expo, New York, June 2009.

[MCCT09] M. Makar, C. Chang, D. Chen, and S. Tsai. Compression of Image Patches for Local Feature Extraction. In ICASSP, Taipeh, April 2009.

[MCUP02] J. Matas, O. Chum, M. Urban, and T. Pajdla. Robust wide baseline stereo from maximally stable extremal regions. In British Machine Vision Conference, volume 1, pages 384-393, 2002.

[MCUP04] J. Matas, O. Chum, M. Urban, and T. Pajdla. Robust wide-baseline stereo from maximally stable extremal regions. Image and Vision Computing, 22(10):761767, September 2004.

$\left[\mathrm{MDS}^{+} 06\right]$ E. Mouragnon, F. Dekeyser, P. Sayd, M. Lhuillier, and M. Dhome. Real time localization and 3D reconstruction. In $C V P R$, pages I: 363-370, 2006.

[Mic12] Microsoft Street-Side views. http://www.bing.com/maps/, Last accessed in 2012.

[ML09] M. Muja and D.G. Lowe. Fast approximate nearest neighbors with automatic algorithm configuration. In Int. Conf. on Comp. Vision Theory and Applications, Lisboa, Portugal, February 2009.

[MS04] K. Mikolajczyk and C. Schmid. Scale \& affine invariant interest point detectors. Int. Journal of Comp. Vision, 60(1):63-86, January 2004.

[MS05] K. Mikolajczyk and C. Schmid. A performance evaluation of local descriptors. IEEE Trans. on Pattern Analysis and Machine Intelligence, 27(10):1615-1630, October 2005.

$\left[\mathrm{MSC}^{+} 09\right] \quad$ C. Mei, G. Sibley, M. Cummins, P. Newman, and I. D. Reid. A constant-time efficient stereo SLAM system. In $B M V C$, pages xx-yy, 2009.

$\left[\mathrm{MTC}^{+} 10\right]$ R. Minetto, N. Thome, M. Cord, J. Fabrizio, and B. Marcotegui. Snoopertext: A multiresolution system for text detection in complex visual scenes. In ICIP, pages $3861-3864,2010$.

$\left[\mathrm{MTS}^{+} 05\right] \quad$ K. Mikolajczyk, T. Tuytelaars, C. Schmid, A. Zisserman, J. Matas, F. Schaffalitzky, T. Kadir, and L.V Gool. A comparison of affine region detectors. Int. J. Comput. Vision, 65:43-72, 2005.

[NNB04] David Nistér, Oleg Naroditsky, and James Bergen. Visual odometry. Computer Vision and Pattern Recognition, IEEE Computer Society Conference on, 1:652659, 2004.

[NS06] D. Nistér and H. Stewénius. Scalable recognition with a vocabulary tree. In CVPR, pages 2161-2168, 2006.

[NS08] D. Nistér and H. Stewénius. Linear time maximally stable extremal regions. In $E C C V$, pages 183-196, 2008.

[NST05] G Navarro, E Sutinen, and J Tarhio. Indexing text with approximate q-grams. Journal of Discrete Algorithms, 3:157-175, 2005.

[NWSS11] G. Nützi, S. Weiss, D. Scaramuzza, and R. Siegwart. Fusion of imu and vision 
for absolute scale estimation in monocular slam. Journal of Intelligent $\mathcal{E}$ Robotic Systems, 61(1):287-299, 2011.

[Ots75] N. Otsu. A threshold selection method from gray-level histograms. Automatica, 11(285-296):23-27, 1975.

[OWAB07] S. Ober, M. Winter, C. Arth, and H. Bischof. Dual-Layer Visual Vocabulary Tree Hypotheses For Object Recognition. In IEEE Int. Conf. on Image Processing, San Antonio, October 2007.

$\left[\mathrm{PCI}^{+} 07\right] \quad$ J. Philbin, O. Chum, M. Isard, J. Sivic, and A. Zisserman. Object retrieval with large vocabularies and fast spatial matching. In IEEE Int. Conf. Comp. Vision Pattern Recognition, Minneapolis, June 2007.

$\left[\mathrm{PCI}^{+} 08\right] \quad J$. Philbin, O. Chum, M. Isard, J. Sivic, and A. Zisserman. Lost in Quantization: Improving Particular Object Retrieval in Large Scale Image Databases. In IEEE Int. Conf. Comp. Vision Pattern Recognition, Anchorage, June 2008.

[PCM09] M. Perd'och, O. Chum, and J. Matas. Efficient representation of local geometry for large scale object retrieval. In IEEE Int. Conf. Comp. Vision Pattern Recognition, Miami, June 2009.

[PCN10] I. Posner, P. Corke, and P. Newman. Using text-spotting to query the world. In Int. Conf. on Intelligent Robots and Systems, Taipei, October 2010.

[RAK09] P. Robertson, M. Angermann, and B. Krach. Simultaneous localization and mapping for pedestrians using only foot-mounted inertial sensors. In Proceedings of the 11th international conference on Ubiquitous computing, pages 93-96. ACM, 2009.

[RD06] E. Rosten and T. Drummond. Machine learning for high-speed corner detection. Computer Vision-ECCV 2006, pages 430-443, 2006.

[RL08] Konrad Rieck and Pavel Laskov. Linear-Time Computation of Similarity Measures for Sequential Data. Journal of Machine Learning Research, 9:23-48, 2008.

[RRKB11] E. Rublee, V. Rabaud, K. Konolige, and G. Bradski. ORB: an efficient alternative to SIFT or SURF. In IEEE International Conference on Computer Vision (ICCV), pages 2564-2571, Barcelona, Spain, 2011. IEEE.

[SAH08] Chanop Silpa-Anan and Richard Hartley. Optimised KD-trees for fast image descriptor matching. In IEEE Int. Conf. Comp. Vision Pattern Recognition, Anchorage, June 2008.

[SBS07] G. Schindler, M. Brown, and R. Szeliski. City-scale location recognition. In IEEE Int. Conf. Comp. Vision Pattern Recognition, Minneapolis, June 2007.

[Sha48] Claude E. Shannon. A mathematical theory of communication. The Bell system technical journal, 27:379-423, 1948.

[SK08] A. Srivastav and J. Kumar. Text detection in scene images using stroke width and nearest-neighbor constraints. In TENCON 2008 - 2008 IEEE Region 10 Conference, pages $1-5,2008$.

[SMD10] H. Strasdat, J. Montiel, and A.J. Davison. Real-time monocular SLAM: Why filter? In Robotics and Automation (ICRA), 2010 IEEE International Conference on, pages 2657-2664. IEEE, 2010. 
[Smi07] R. Smith. An overview of the Tesseract OCR engine. In Int. Conf. on Document Analysis and Recognition, Brazil, September 2007.

[SNDC07] K. Subramanian, P. Natarajan, M. Decerbo, and D. Castanon. Character-stroke detection for text-localization and extraction. In $I C D A R$, volume 1 , pages 33 $-37,2007$.

[SPT11] P. Shivakumara, T.Q. Phan, and C.L. Tan. A laplacian approach to multioriented text detection in video. IEEE Trans. Pattern Anal. Mach. Intell., 33(2):412 -419, 2011.

[ST94] J. Shi and C. Tomasi. Good features to track. In Computer Vision and Pattern Recognition, 1994. Proceedings CVPR'94., 1994 IEEE Computer Society Conference on, pages 593-600. IEEE, 1994.

[Str12] Julian Straub. Visual localization based on binary features. Master's thesis, Techniche Universität München, 2012.

[SZ03] J. Sivic and A. Zisserman. Video Google: A text retrieval approach to object matching in videos. In IEEE Int. Conf. on Comp. Vision, pages 1470-1477, Nice, October 2003.

$\left[\mathrm{TBB}^{+} 99\right] \quad$ S. Thrun, M. Bennewitz, W. Burgard, A.B. Cremers, F. Dellaert, D. Fox, D. Hahnel, C. Rosenberg, N. Roy, J. Schulte, et al. Minerva: A second-generation museum tour-guide robot. In IEEE International Conference on Robotics and Automation, 1999.

[TCC $\left.{ }^{+} 10 \mathrm{a}\right]$ G. Takacs, V. Chandrasekhar, D. Chen, S. Tsai, R. Grzeszczuk, and B. Girod. Unified Real-Time Tracking and Recognition with Rotation-Invariant Fast Features. In IEEE Int. Conf. Comp. Vision Pattern Recognition, San Francisco, June 2010.

$\left[\mathrm{TCC}^{+} 10 \mathrm{~b}\right]$ S. Tsai, D. Chen, V. Chandrasekhar, G. Takacs, N.M. Cheung, R. Vedantham, R. Grzeszczuk, and B. Girod. Mobile product recognition. In Proc. ACM Multimedia 2010, 2010.

[TCGP09] D.N. Ta, W.C. Chen, N. Gelfand, and K. Pulli. SURFTrac: Efficient tracking and continuous object recognition using local feature descriptors. In IEEE Int. Conf. Comp. Vision Pattern Recognition, Miami, June 2009.

$\left[\mathrm{TXG}^{+} 08\right]$ G. Takacs, Y. Xiong, R. Grzeszczuk, V. Chandrasekhar, W. Chen, L. Pulli, N. Gelfand, T. Bismpigiannis, and B. Girod. Outdoors augmented reality on mobile phone using loxel-based visual feature organization. In Proc. ACM Multimedia Information Retrieval, pages 427-434, 2008.

[Ukk92] Esko Ukkonen. Approximate string-matching with q-grams and maximal matches. Theoretical Computer Science, 92:191-211, 1992.

[Ull77] J R Ullmann. A binary n-gram technique for automatic correction of substitution, deletion, insertion and reversal errors in words. The Computer Journal, 20:141$147,1977$.

[vGVSG10] J van Gemert, C Veenman, A Smeulders, and J Geusebroek. Visual Word Ambiguity. IEEE Trans. on Pattern Analysis and Machine Intelligence, 32(7):1271 - 1283, July 2010.

[WKIS09] Zhong Wu, Qifa Ke, M. Isard, and Jian Sun. Bundling features for large scale 
partial-duplicate web image search. In IEEE Int. Conf. Comp. Vision Pattern Recognition, Miami, June 2009.

[WMB99] I.H. Witten, A. Moffat, and T.C. Bell. Managing gigabytes: compressing and indexing documents and images. Morgan Kaufmann, 1999.

[WS11] S. Weiss and R. Siegwart. Real-time metric state estimation for modular visioninertial systems. In 2011 IEEE Int'l Conference on Robotics and Automation (ICRA), pages 4531-4537, May 2011.

[YHGZ05] Q. Ye, Q. Huang, W. Gao, and D. Zhao. Fast and robust text detection in images and video frames. Image Vision Comput., 23:565-576, 2005.

[YWY07] J. Yuan, Y. Wu, and M. Yang. Discovery of collocation patterns: from visual words to visual phrases. In IEEE Int. Conf. Comp. Vision Pattern Recognition, Minneapolis, June 2007.

[ZZJ00] Y. Zhong, H. Zhang, and A.K. Jain. Automatic caption localization in compressed video. IEEE Trans. Pattern Anal. Mach. Intell., 22(4):385 -392, 2000. 